(1)

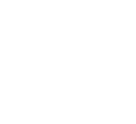

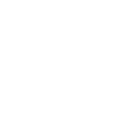<smiles>C1CCC1</smiles>
$\overline{-}$ . 


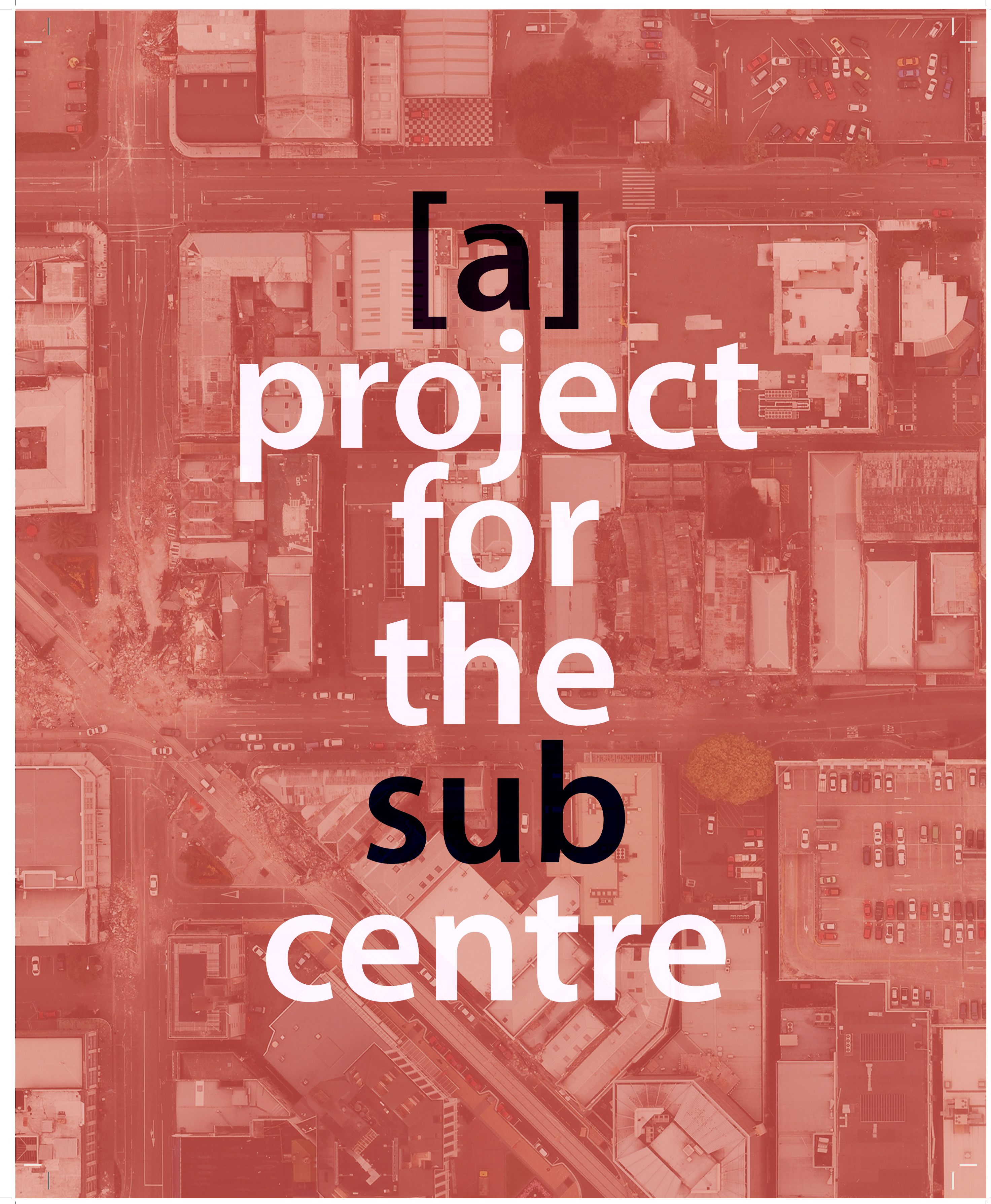




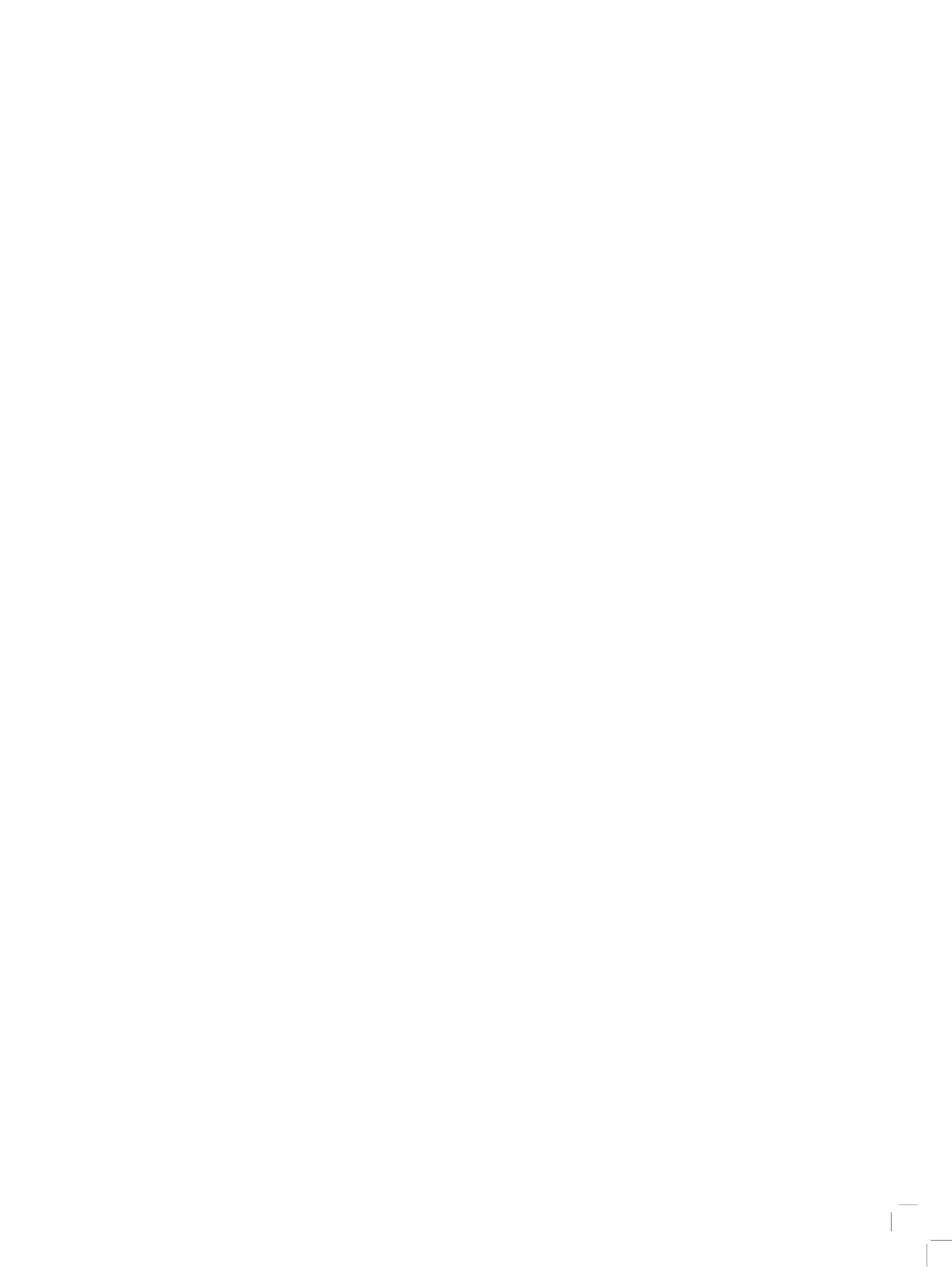




\title{
[a] Project for the Sub-Centre
}

\author{
An Agora Anew \\ (a project for a sub-centre) \\ $+$ \\ Towards the Sub-Centre as \\ Architecture's Project
}

by

Grace Helen Salisbury Mills

for the degree of Master of Architecture (Professional) 
"From the point of view of form, the type of all the arts is the art of the musician."

- Oscar Wilde 


\section{Acknowledgements}

Firstly I need to thank my parents for their undivided support over five incredibly drawn out years. This support - be it intellectual; emotional, psychological, financial or nutritional - has been absolutely crucial for me to make it through these five these years. Mum, especially, for all the amazing hospitality, Dad, for keeping me positive when I need it most and showing me that, in fact, there is life outside of architecture. Thanks for putting up with my intermittent uselessness and making five years something I surely won't regret.

Secondly I have to thank my non-architecture friends - as I have so fondly referred to them. Thanks for putting up with my periods of absence; my crazed rants, and for keeping me occupied outside of architecture school so very, very well. It made each of the five years worthwhile in their own right. Especially this is directed at: Hanna, Tim, Tina, Claire, Joel, Bailee, Catherine, Robin, James and James.
Thirdly, I have to thank Victoria University for their generous granting of financial support - without which, my final year would have been significantly more difficult - and the other sources of funding which have allowed me to get through the two degrees with a wee bit less skin off my bones. Within the faculty; thanks for Jan and Simon for their on-going support; and to Sam Kebbell for enacting the topic at the heart of my thesis - and instilling doubt, just when I thought I might be getting somewhere (but probably wasn't!).

Last - but absolutely not least - I have to thank my year of fellow students. We have shared probably a few too many moments of insanity; ingenuity; frustration; acceptance; inspiration, and euphoria - and definitely too many 'rehs'. P-Rus, Yokes, KJ, Kilkenny, Skogsy, thanks for putting up with my bird antics. Josie, thanks for the Floridita's breakfasts; Hannah for a ridiculouslygood friendship; and my BOYS Liam and Angus for an excessive amount of disciplinary obsession; digirealm explorations, and architectural memes (which, undoubtedly got us through those final months). 


\section{Abstract}

In the aftermath of the 2011 earthquake, a state of polycentric urbanity was thrust upon New Zealand's second largest city. As the city-centre lay in disrepair, smaller centres started to materialise elsewhere, out of necessity. Transforming former urban peripheries and within existing suburbs into a collective, dispersed alternative to the city centre, these sub-centres prompted a range of morphological, socio-cultural and political transformations, and begged multiple questions: how to imbue these new sub-centres with gravity? How to render them a genuine alternative to the CBD? How do they operate within the wider city? How to cope with the physical and cultural transformations of this shifting urbanscape and prevent them occurring ad lib? Indeed, the success and functioning of the larger urban structure hinges upon a critical, informed response to these sub-centre urban contexts. Yet, with an unrelenting focus on the CBD rebuild - effectively a polycentric denial - little such attention has been granted.

Taking this urban condition as its premise and its provocation, this thesis investigates architecture's role in the emergent sub-centre. It asks: what can architecture do in these urban contexts; how can architecture act upon the emergent sub-centre in a critical, catalytic fashion? Identifying this volatile condition as both an opportunity for architectural experimentation and a need for critical architectural engagement, this thesis seeks to explore the sub-centre (as an idea and actual urban context) as architecture's project: its raison d'etre, impetus and aspiration.
These inquiries are tested through design-led research an initial design question provoking further, broader discursive research (and indeed, seeking broader implications). The first section is a site-specific, design for Sumner, Christchurch. Titled 'An Agora Anew'; this project - both in conception and outcome - is a speculative response to a specific sub-centre condition. The second section 'The Sub-centre as Architecture's Project' explores the ideas provoked by the design project within a discursive framework. Firstly it identifies the sub-centre as a context in desperate need of architectural attention (why architecture?); secondly, it negotiates a possible agenda for architecture in this context through terms of engagement that are formal, critical and opportunistic (how architecture?): enabling it to take a position on and in the sub-centre. Lastly, a critical exegesis positions the design in regards to the broader discursive debate: critiquing it an architectural project predicated upon the idea of the sub-centre.

The implications of this design-led thesis are twofold: firstly, for architecture's role in the sub-centre (especially to Christchurch); secondly for the possibilities of architecture's productive engagement with the city (largely through architectural form), more generally. In a century where radical, new urban contexts (of which the sub-centre is just one) are commonplace, this type of thinking - what can architecture do in the city? - is imperative. 


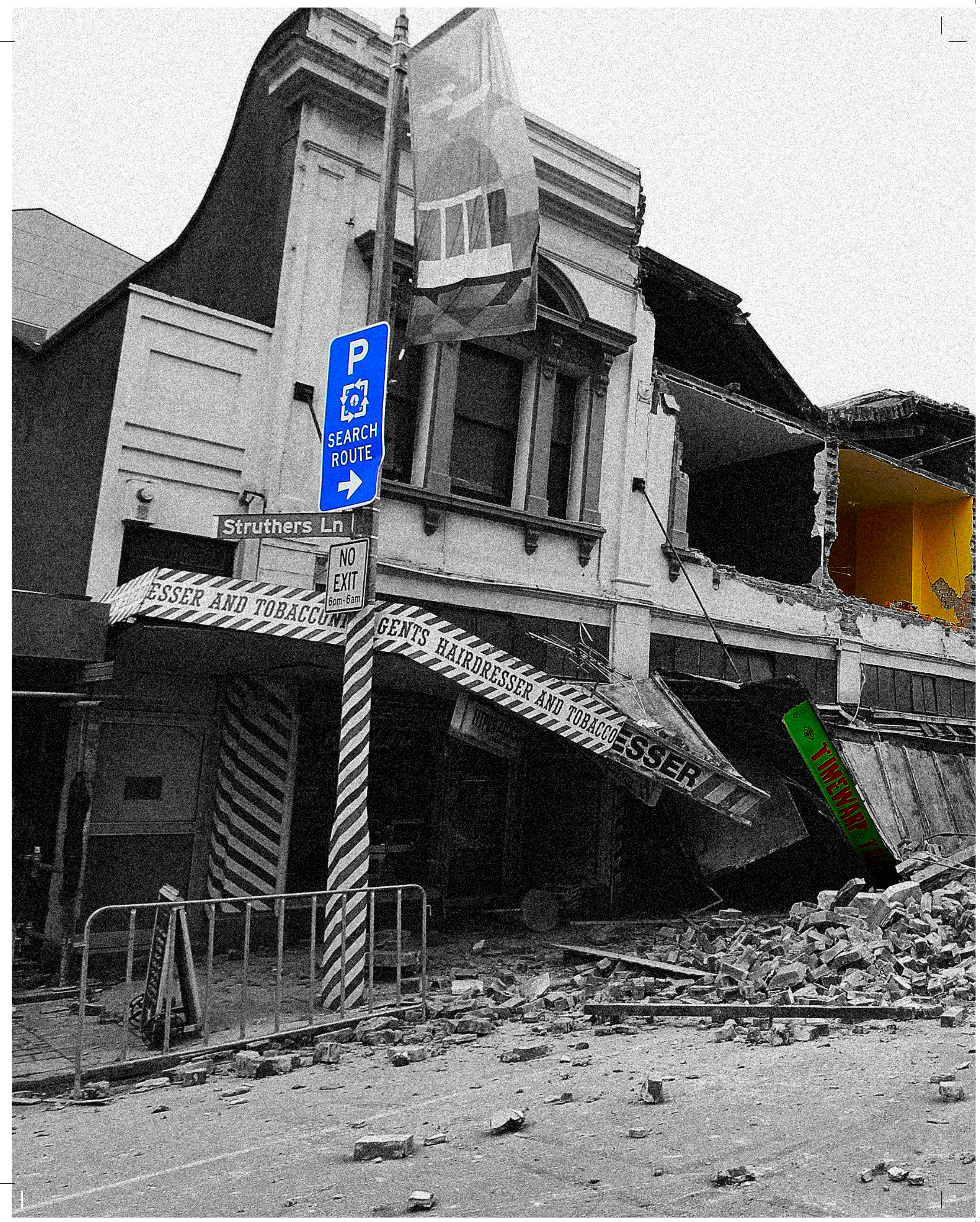


"Indeed,there is a serious link between crisis \& creativity"

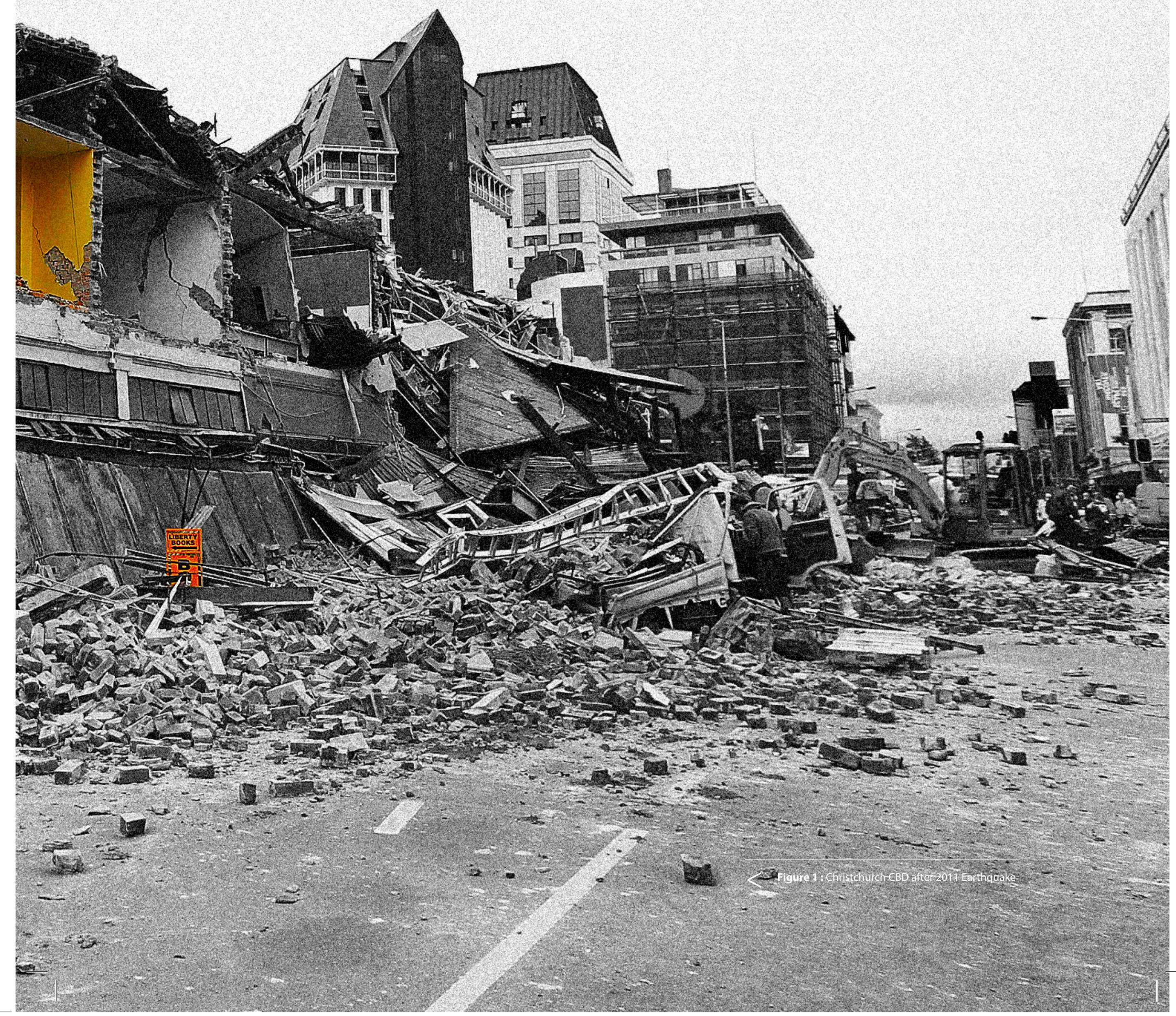




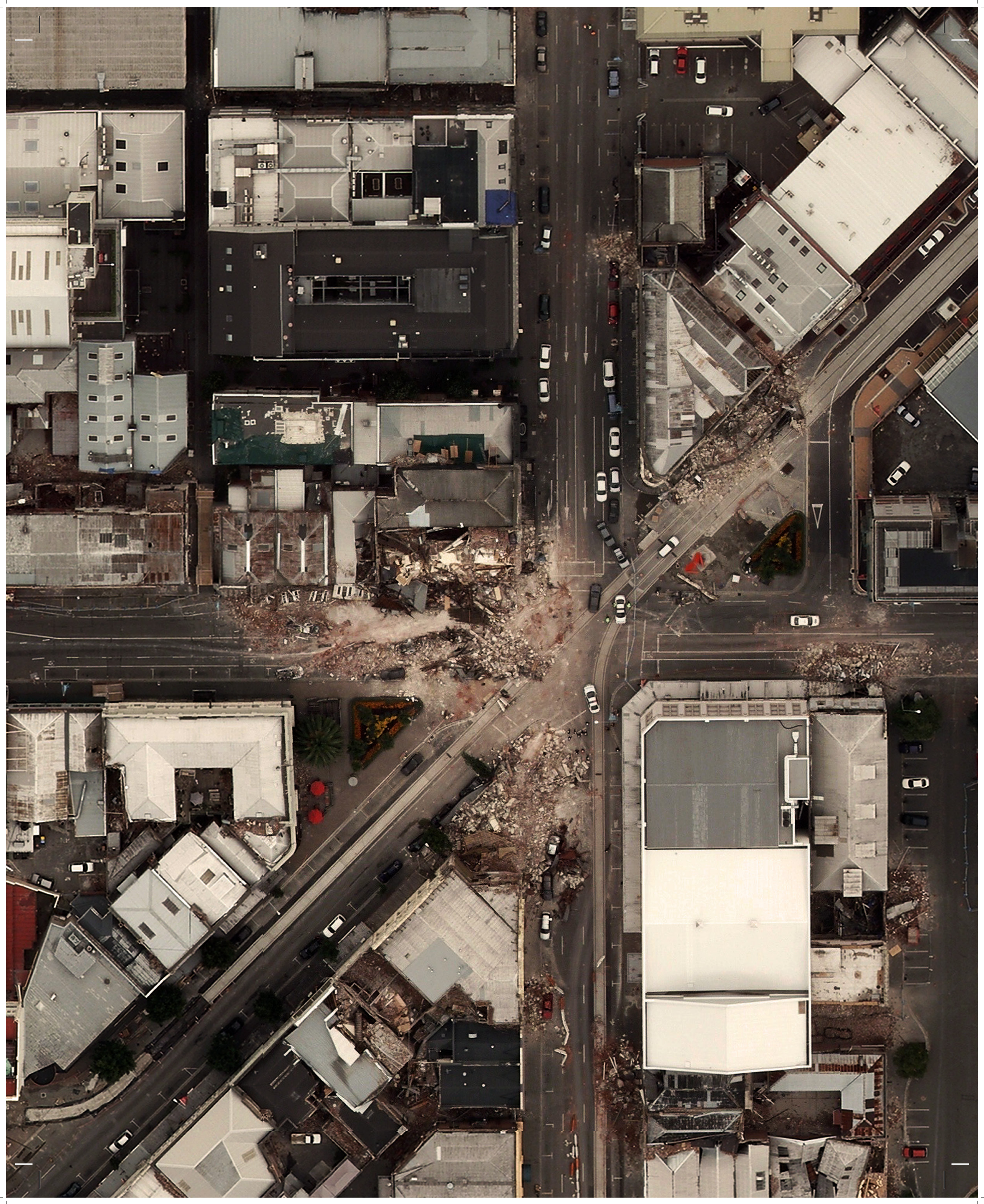




\section{Preface}

The aftermath of the destructive 2011 Christchurch earthquakes coincided neatly with the first forays into this thesis, and the forming of potential topics in an otherwise unbounded array of possibilities (other than my own personal calling).

My intention was to somehow situate this issue with the architectural discipline, and I found a way to do so through the polycentric city: a condition emerging in Christchurch in the wake of a destructed CBD, but one also proliferating at a universal scale, and with intriguing and somewhat-underexplored architectural implications.

This multi-centred urban reality is fairly unprecedented in our young, 'rural-citied' nation, but has been set upon us in full force. On account of this, it seems to warrant explicit, intellectual and immediate discussion, especially in the face of what Tommy Honey has identified as anti-urban sentiment: an unwillingness to engage with the fact that we are, actually, a veryurbanised nation (perhaps, indeed, to detrimental effects). Moreover, there is chance that, given the ubiquity of the poly-centric paradigm, site-specific research into Christchurch's current situation may bear benevolent effects at a more global level. This, to me, must be the point of research: at the least, we should aim be an exemplary model: to set an example if we can.

In addition to this practical beginning - though not coincidently - the conceptions for this thesis also spawned from a personal interest in architecture's multi- facetted relationship to the city: a relationship which in my five years of study has been the most dynamic and provocative area and one which I believe keeps architecture constantly on its toes, propelling the discipline into the most unexpected of places.

As the city continues to assert itself as the true domain of architecture, architecture's role within it politically, socially, culturally, spatially, poetically (the list goes on) - simply cannot receive enough attention. Inadequate exploration can (and has, many times, already) lead to detrimental, flow-on effects, both for the city, and for architecture itself: presenting back-toback opportunities and challenges for us to take on. Architecture never stops yearning to satiate, and the city never lets it: so the latter serves as the source of most criticism, frustration and disillusion regarding the former. Regardless, we must persevere, explore, and question.

And so emerged, between this very real and immediate problem and a broader, on-going disciplinary issue, an underlying endeavour to bring the practical and discursive aspects of the discipline into dialogue: as only appropriate at the end of a professional-academic degree - as well as for a Masters Thesis predicated on taking a position through 'design-research'.

While in many ways, the project is idealistic, reflecting my own, university-yielding views about architecture's potentials as a creative-cultural and critical medium, I believe it may also offers an infinitesimal piece of knowledge to an issue of unwavering relevance; and furthermore, that it may push some boundaries, somewhere, in a small, acutely-angled direction. If this is all I can do, then it has been a five years well served.

$<$ Figure 2: a diagram showing the 'real' and 'disciplinary' 


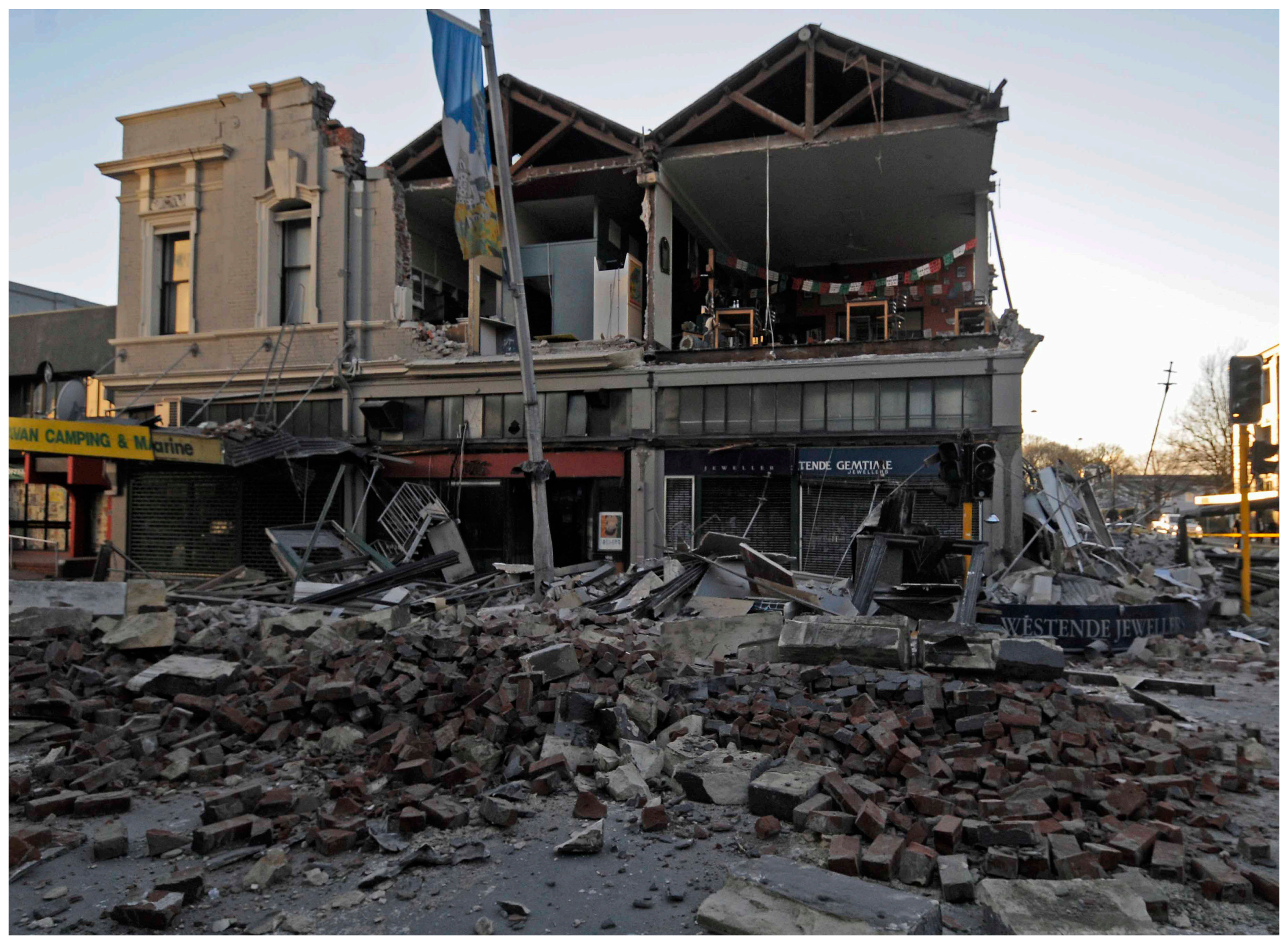

Figures $3+4$ : Christchurch's CBD endured large scale destruction from the 2011 earthquakes, with vast

implications for the wider city as it was essentially forced to $>$ decentralize, and relocate to suburbs. 


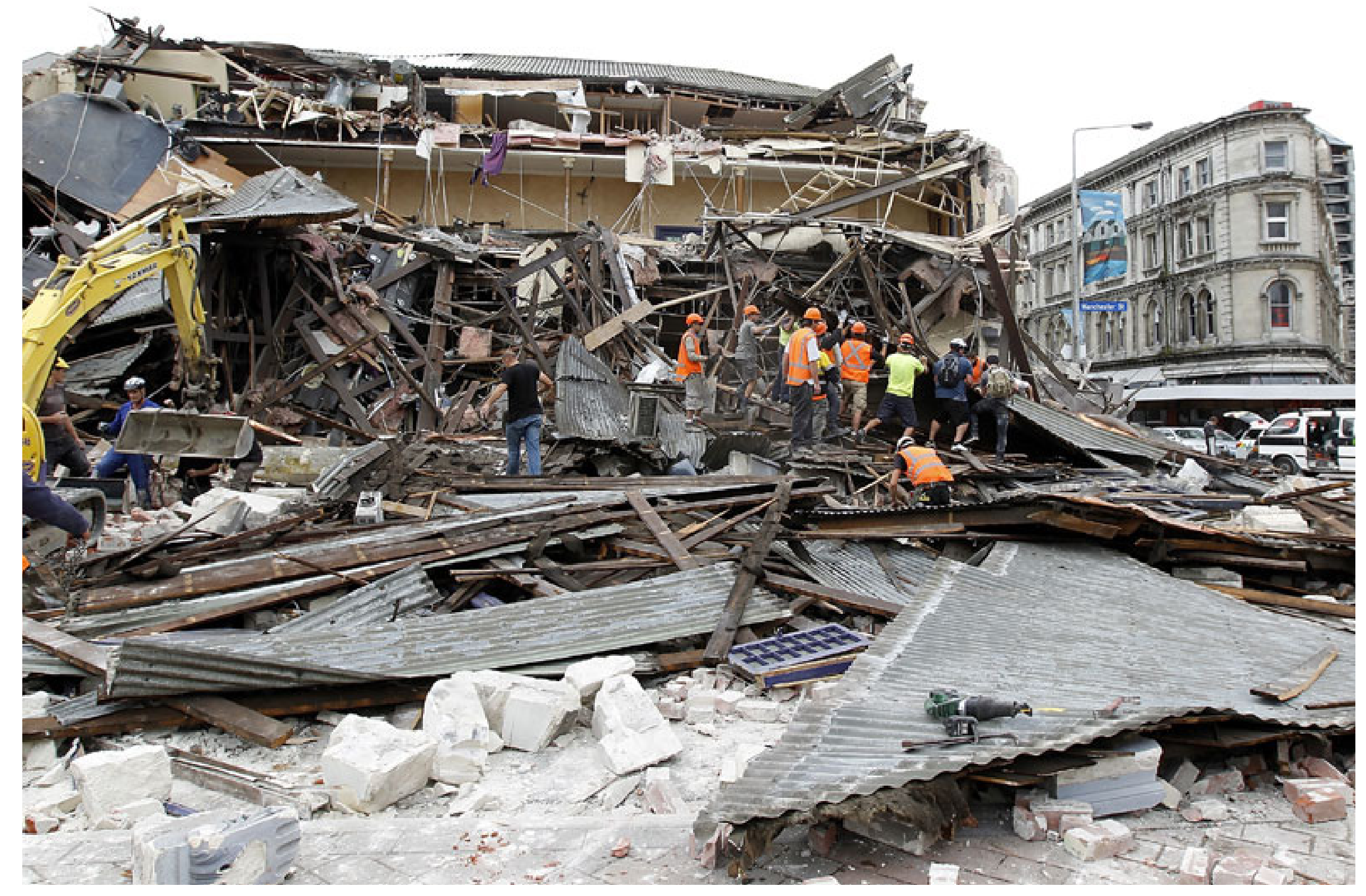






\section{Contents}




\section{What Suburban Lights? elucidating the gap for design- research}

One year on from the 2011 Christchurch earthquake, a reporter remarked of the city's current predicament "the empty city centre is a dark smudge, [surrounded by] suburban lights"1 ${ }^{\prime \prime}$ This statement acutely betrayed not only the gravity of the city's immediate situation, but effortlessly revealed Christchurch's emerging urban reality in its utmost irony: the notion of 'suburban lights' presenting a plain paradox to a country whose suburbs are far from well lit, but are more typically subservient to - and indeed lit by - a city centre (in a multitude of senses: culturally, socially, politically and economically). Albeit inadvertently, these juxtaposing words resonated with a surprising potency - and moreover, hinted at a dire urban reality.

Indeed, though the substantial destruction of the city-centre produced a "darkness at the heart of Christchurch" ${ }^{2}$, and thrust New Zealand's second largest city into a state of polycentricity - it soon became apparent that a deeply ingrained monocentric urban existence rendered this a troublesome term to come to grips with. While a polycentric model plagued initial discussions around the city's recovery, more recently focus has been decisively directed towards the CBD's rebuild: resisting the opportunities of a dispersed multi-centred urban structure, and probing wide and varied criticism ${ }^{4}$. Nevertheless, the structure of the city has fundamentally changed - and is continuing to do so, ad lib - as urban activity necessarily relocates in absence of the centre. In this regard, a polycentric reality is becoming: well-lit or otherwise.

There is abundant precedent for this imminent - or rather latent - state of urbanity, outside of our antipodean setting. The polycentric city has proliferated in urban regions globally in recent decades, and the development of multiple, networked centres (sub-centres) within a single urban region is seen to present vast urban possibilities and opportunities: social, economic and ecological in nature.

Still, this urban model, and particularly its sub-centre constituents, is not without problems. Given that these urban spaces are often dominated by economic and functional imperatives, these priorities often feature disproportionately within strategies and visions to develop sub-centres. Compounded with the rate at which these previously nonurban environments are transformed, this often does not bode well for their spatial and formal materialisation, or, in due course, their gravity and enduring quality as a viable citycentre alternative. In a worst case scenario, this has given rise to sub-centres with little affectation for a veritable, socio-cultural or political centrality. Especially in the event where the CBD is barely present; the emergence of these culturally or sociallyimpotent urban spaces is disconcerting: both at their own scale and that of the polycentric city of which they are a part.

\section{A problem for architecture}

So where is the loophole? Given the fact that sub-centres typically emerge within urban peripheries, and formerly monofunctional suburbs, they are largely brought to bear through morphological transformations at the scale of built form: especially as density, diversity and urban activity are catered for. Here, in the multi-facetted transformation from suburb to sub-centre, architecture is clearly implicated. Yet, beyond the mere facilitation of densities and mixtures of urban functions (living, working, commercial), the possibility of architecture doing something in this somewhat-confused urban context has barely been broached. 

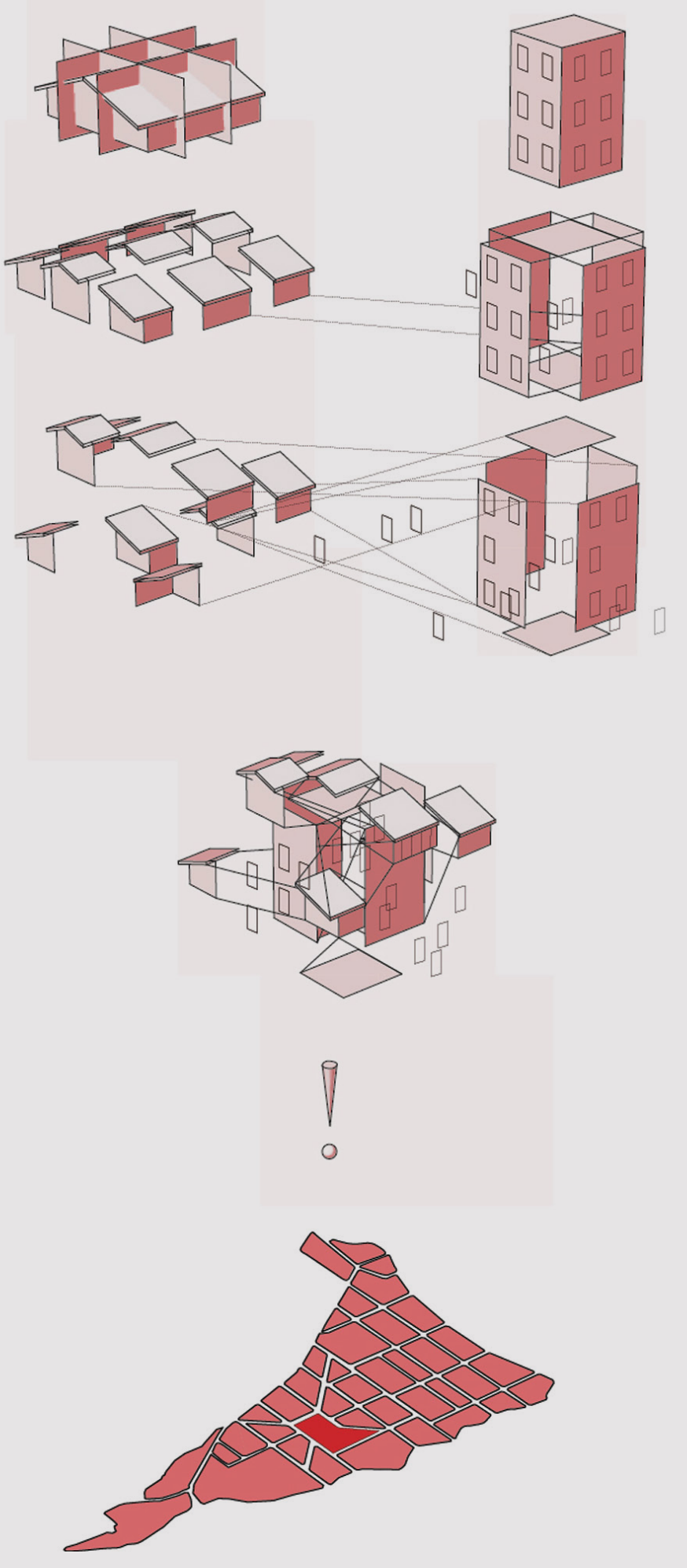

Figure 5: An early parti diagram reflecting the initial designresearch questions: What does the synthesis of living (left) and working (right) cultures in the emergent sub-centre mean for architecture? What might architectural 'do' - through form, for architecture? What might architectural 'do' - through form, for
example - to engage the the sub-centre as a an alternative to the CBD? (shown here, Sumner, Christchurch) 


\section{Opportunity for research}

This thesis sees here a plain gap for architectural designresearch. In order to mitigate sub-centres materialising with little sense of cultural, social, political potency, or indeed gravity, it hypothesizes that architecture may not only have a role, but, potentially, a critical urban agenda.

Here, numerous questions are evoked: how might architecture be instrumental - spatially, formally - upon this precarious emerging urban context? How might architecture respond to this emerging urban condition actively and critically - in so doing, attest to (or engender) its significance beyond economic aspects? As it caters for living and working, and urban programmes coming together in the sub-centre - inducing conditions of density and diversity - how can architecture imbue a condition of centrality in this context? (figure 5). Back to the original provocation - how might suburban lights be created that engender a veritable alternative to the CBD, in its absence?

\section{Broader research implications}

From this somewhat intuitive and contextual problem arose a larger array of questions, further probing the possibility of architecture's urban instrumentality, and the reasonable ambitions of architectural design in this context. The lack of focus on architecture's critical agenda in the sub-centre reflects a wider, recent architectural tendency to neglect the city - and likewise, architecture's engagement with larger urban issues (or ideas) to be held as beyond architecture's scope.

Contemporarily, however, forays into architecture's strategic, productive engagement with the city have resurfaced in design discourse. These reflect the view that "architecture needs to rethink its relation to the city"15 and its action not just in, but upon an urban context. Particularly, these efforts explore the relationship between architectural form and the city, and seek formal operations that may allow architecture to re-engage contemporary urban contexts. However, these have seldom been explored in relation to the sub-centre directly.

This fertile discussion thus presented an opportunity for broader research into architecture's engagement with and instrumental role in regards to the sub-centre. Moreover, it offered implications beyond site-specific design, and beyond a Christchurch context.

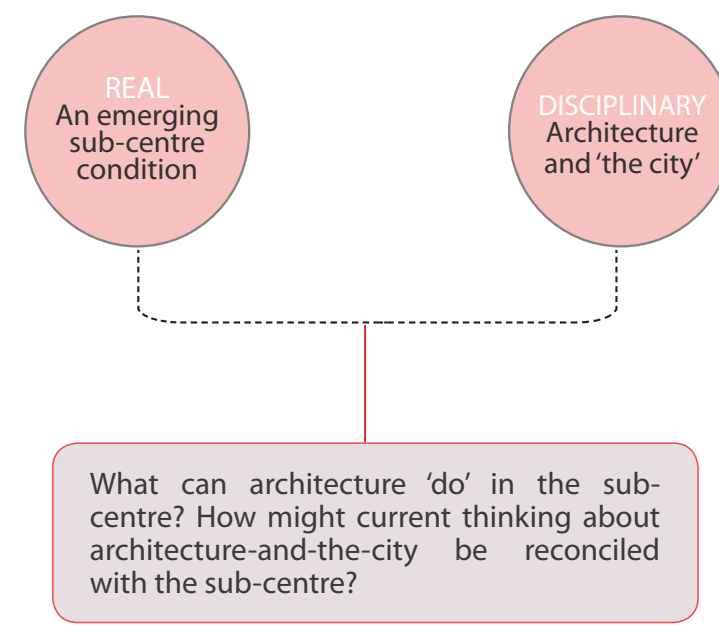

Figure 6: a diagram showing the 'real' and 'disciplinary' components that may be understood to form the basis of the research questions for this thesis.

\section{Research questions and objectives}

Conflating, therefore, what is a real and very imminent (contextdriven) situation with a broader, on-going discursive debate (architecture's urban instrumentality), this thesis sets out to consider the emergent urban sub-centre as a context within which to re-evaluate architecture's productive and instrumental relationship to the city (figure 6). It asks:

What does the sub-centre mean for architecture? In what capacity does the sub-centre require architecture? What can architecture do in the emergent urban sub-centre? How can current thinking about architecture-and-the-city be mobilised within the sub-centre as a distinct urban context? What can this offer to related design discourse?

In relation to these questions, three main objectives were formulated:

- To investigate architecture's critical, instrumental role in and upon an emergent sub-centre through site-specific design (a way of producing).

- To extend current (design) discourse about architecture's role in the emergent sub-centre: both by revealing the subcentre as a problem for architecture (a way of thinking) and by exposing possible modes of operation (a way of working) that may allow architecture's active engagement with the sub-centre.

- To critically reflect upon the design outcome against the broader discursive argument: exposing both its implications and limitations as a project for the sub-centre. 


\section{On design-led research as a methodology}

This thesis adopts a specific position on design-research that is design-led. This reflects the fact that the original provocation - a clear opportunity for architectural design - led to a more discursive investigation, and into broader implications for design, more generally. Research is understood to be embedded within the design outcome; the broader discursive argument it prompts; and the design processes (as reflected upon, critically). In this way, the design is not a'test' to reveal the research, but is both the research itself as well as a stimulant of broader argument. In short, this thesis may be seen as problem eliciting design, design prompting discourse and, finally, discourse enabling reflection.

The research is presented accordingly: design first, a broader discursive argument second (figure 7). Without suggesting the two were separately conducted, this reflects their dialogue as inextricable and complementary modes of research: but also that the former (real, design-based) largely prompted the latter (discursive). While these formulated such that they can be read separately - as a design project, and as a research paper - is it hoped that the reader will flick between the two as desired.

\section{Chapter summary}

The first design section comprises the design-outcome for the emergent sub-centre of Sumner, Christchurch. In conception and outcome, this project responds to the initial provocation: what is the role of architecture in the sub-centre? How can it engage the sub-centre as an urban condition? Titled 'An Agora Anew: a project for a sub-centre' this speculative project - a live-work Agora in Sumner, is presented as a finalised project.

The second section is a research paper which demystifies the initial provocations in a broader discursive argument, beyond the design's immediate context. Titled 'Towards the Sub-Centre as Architecture's Project', this paper follows a problem-solution format. Firstly, it argues that the emergent sub-centre is a context begging for architectural attention: and in which architecture's critical urban agenda needs re-thinking (why architecture?). Secondly, it addresses how contemporary thinking about architecture and the city can be mobilised with the sub-centre context (how architecture?) This paper focuses on the role as architectural form as a means for architecture to reengage the sub-centre.
Lastly, the exegesis brings the design and discursive argument into dialogue. By introducing the strategies used, it discusses the design project holistically, both according to its initial objective and the offerings of the research: each interested in architecture's relationship to the sub-centre. The exegesis is an interpretation of the design project, as a means to understand its implications. It is as much a critique as a means to re-frame the design-thinking in relation to the broader question at the heart of this thesis: what can architecture do in the city?

\section{Scope of research}

This thesis deals fundamentally with architecture's relationship to the sub-centre. The scope of the design investigation is limited to the particular sub-centre context of Sumner, Christchurch and tests the research questions against a singular design response.

The discursive component of the research addresses the subcentre as a more general urban condition: conflating urban and architectural notions into a singular argument. While on this one hand this wider scope means the research may have a wider set of implications, it also means it is somewhat limited in the amount of depth or detail it can go into. In this way, certain assumptions/generalisations are made about the nature of this urban condition, and the way architecture might engage it. Particularly for the former, there is an emphasis on image or idea of the sub-centre, and for the latter, there is an emphasis on a formal response. These represent two of many ways in which these urban and architectural issues could be brought into dialogue.

Collectively, the design and discursive argument are limited to the scope of a Masters design-led thesis. 


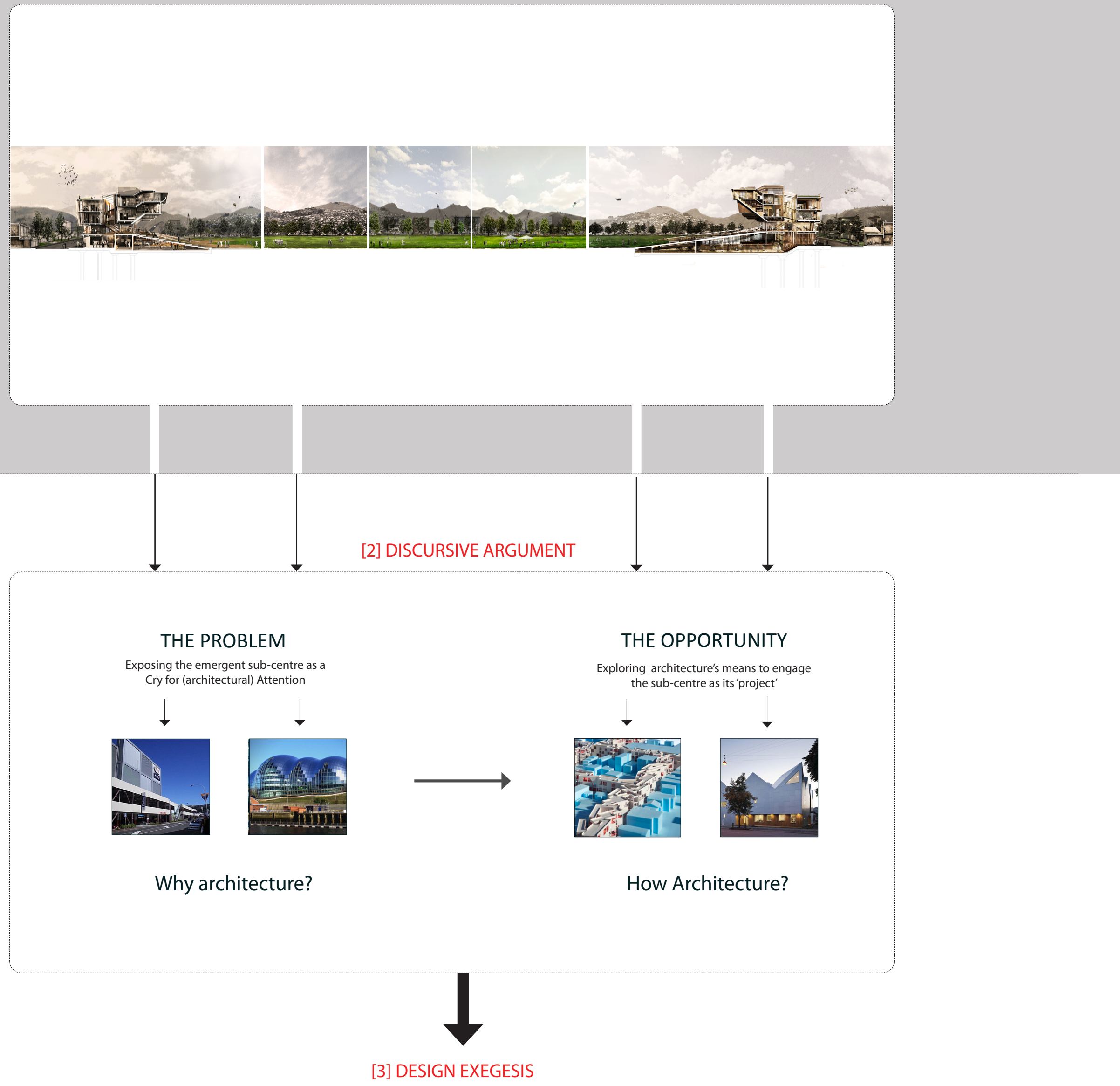

A critical reflection on the design as a project on the sub-centre

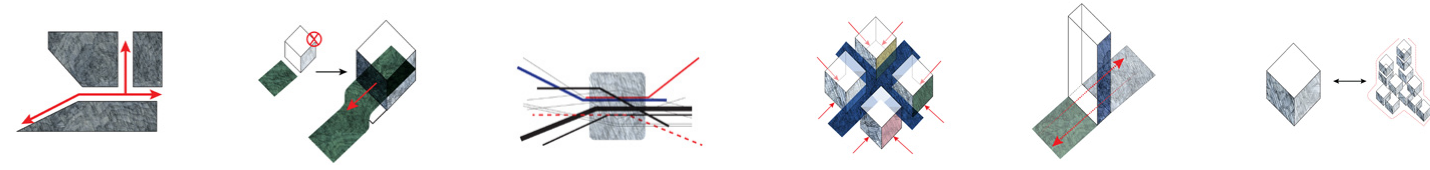




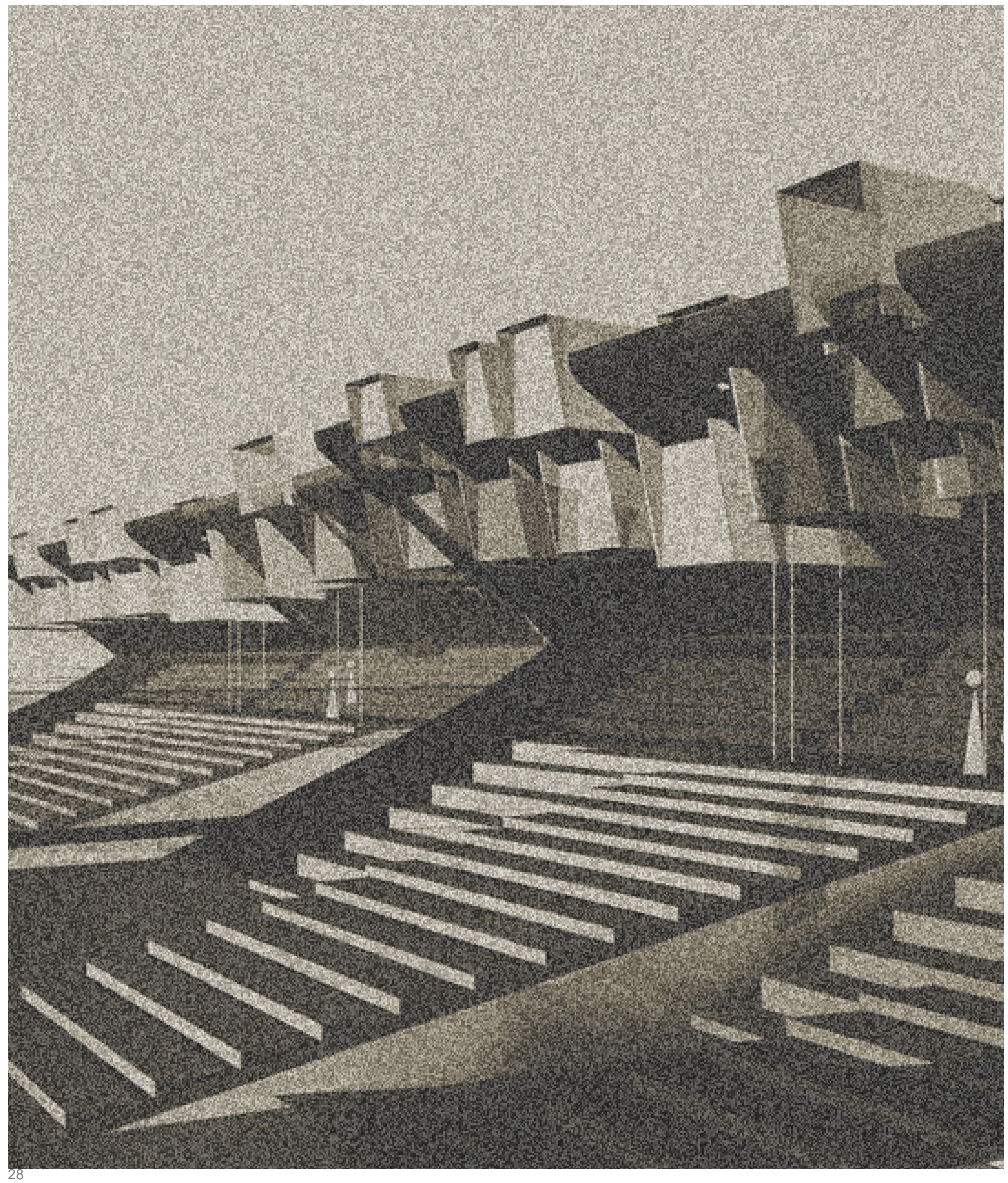




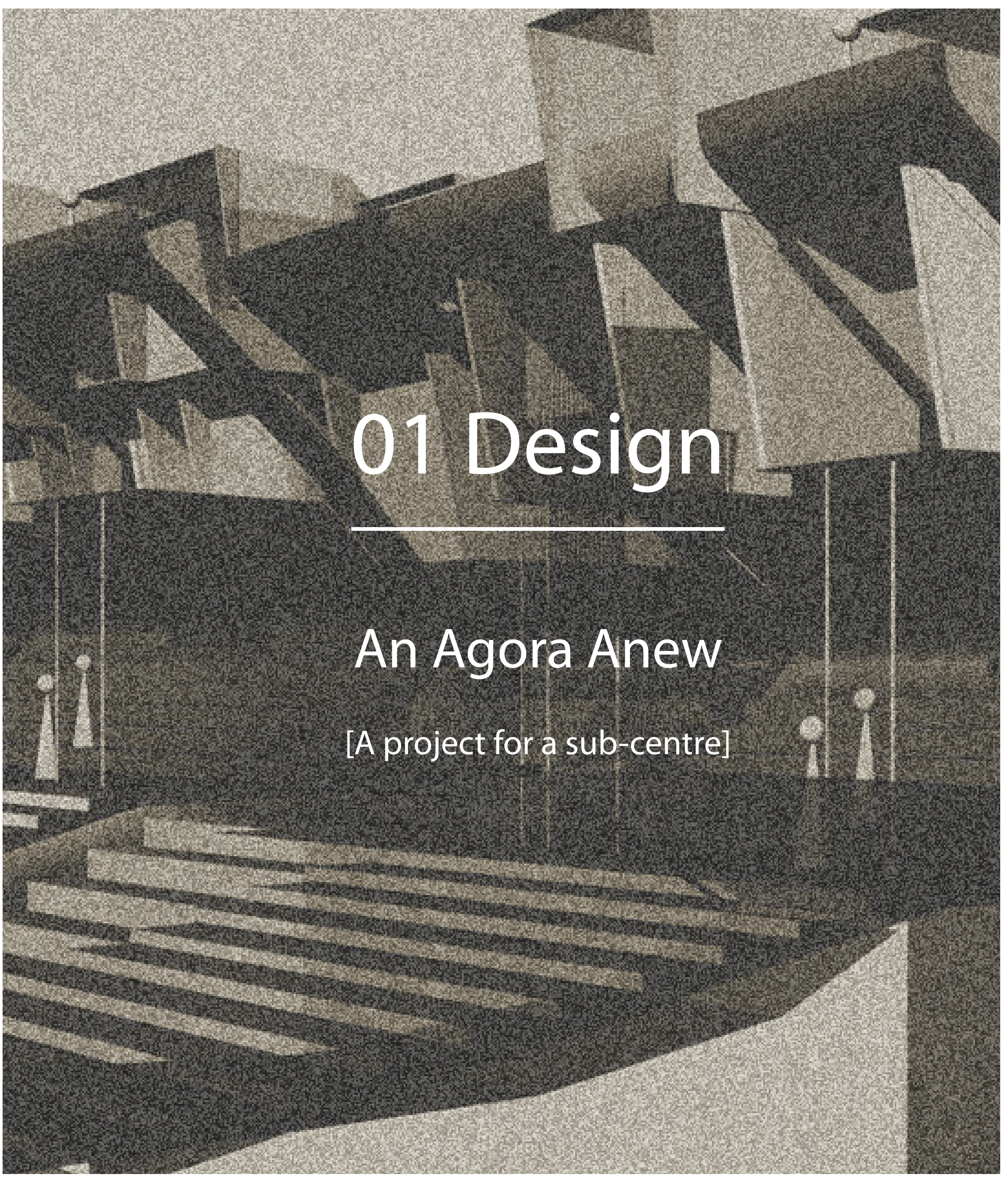



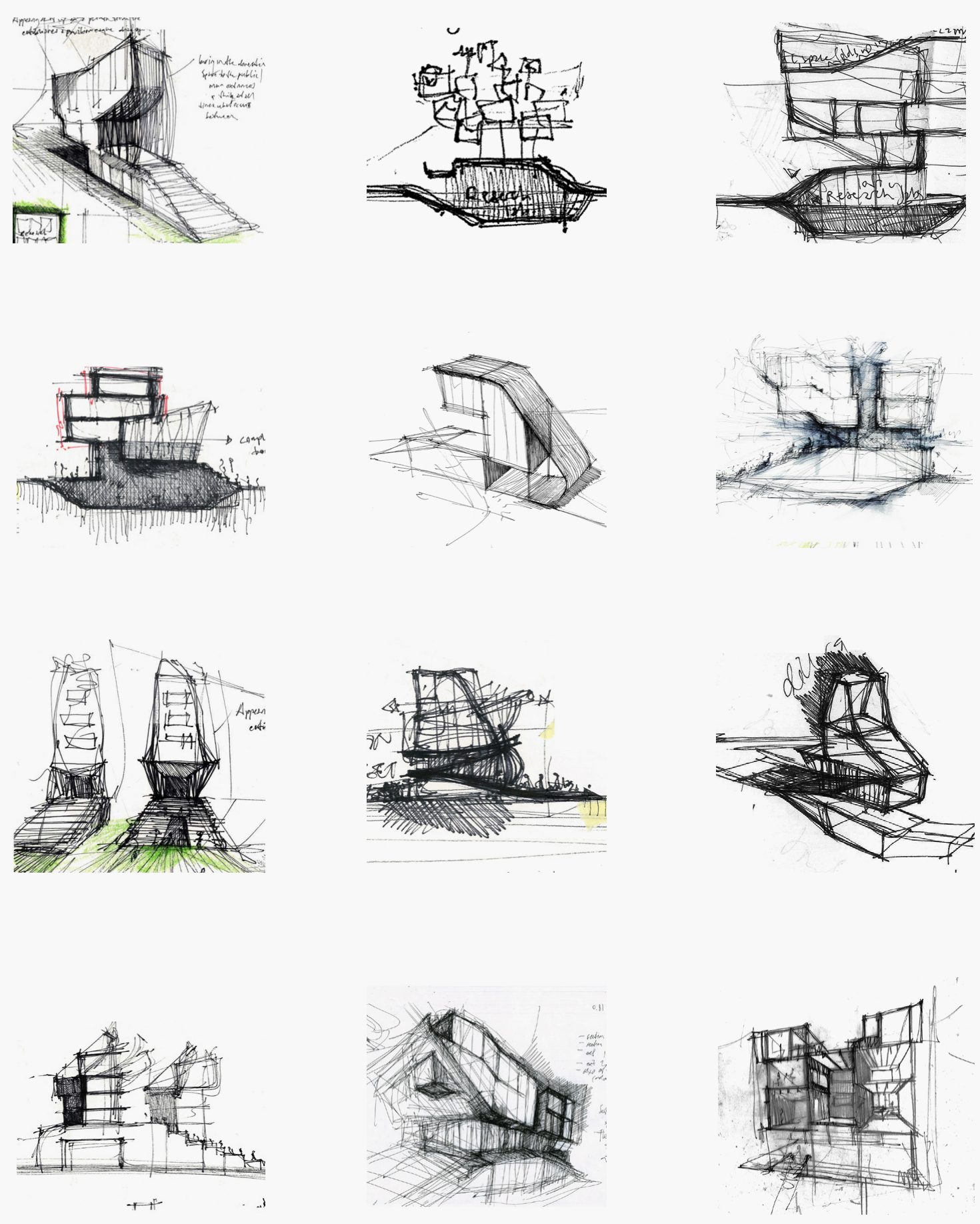

Figure 9: A composite image comprising sketches completed 


\section{Design Foreword}

In accordance with the objectives of this design-led research, the design-project is presented in a twodistinct parts: firstly, below, as a 'finalised' design; secondly - in the design exegesis (chapter 3)- as a series of experiments, processes and strategies, which, while indeed informing the design, were reflected on retrospectively, rather than determined a priori. In so doing, rather than premising the project with a chronological but ultimately haphazard set of design processes, this section gives primacy to what the architecture does and is (its ontology); rather than why and how it does it (its epistemology).

This ordering is tactical: while indeed, the conception and reasoning of the design-output are no doubt eluciated by the preceding processes; it is equally the case that the final design allows critical (and useful) sense to be made out of the design process (which may not be possible otherwise). It is this second process that constitutes, in the author's opinion, the purpose, and indeed the potency of design-led research: and on this account the process is not to be given precedence in this presentation, but is seen as more valuable when presented after the fact (to understand the design).

In dealing with the design first in this 'final' and ontological manner, it is necessary to note that nothing about this project was preconceived. Aside from the primary objective outlined in the introduction - to investigate an architectural response to an emergent subcentre condition - the 'brief', programme and constraints of the design evolved simultaneously to the designresponse itself. To this end, to premise the design with a series criteria or a preconceived methodology (to be tested) would be a falsification of its true becoming: in fact, a near-constant, dialogue between design-intent and design-output.

In the present chapter, the conception and criteria of the design should be seen as inextricable from the design itself - a self-discovering, self-fulfilling, selfreflexive and self-hypothesising project - by design. 

SITE 


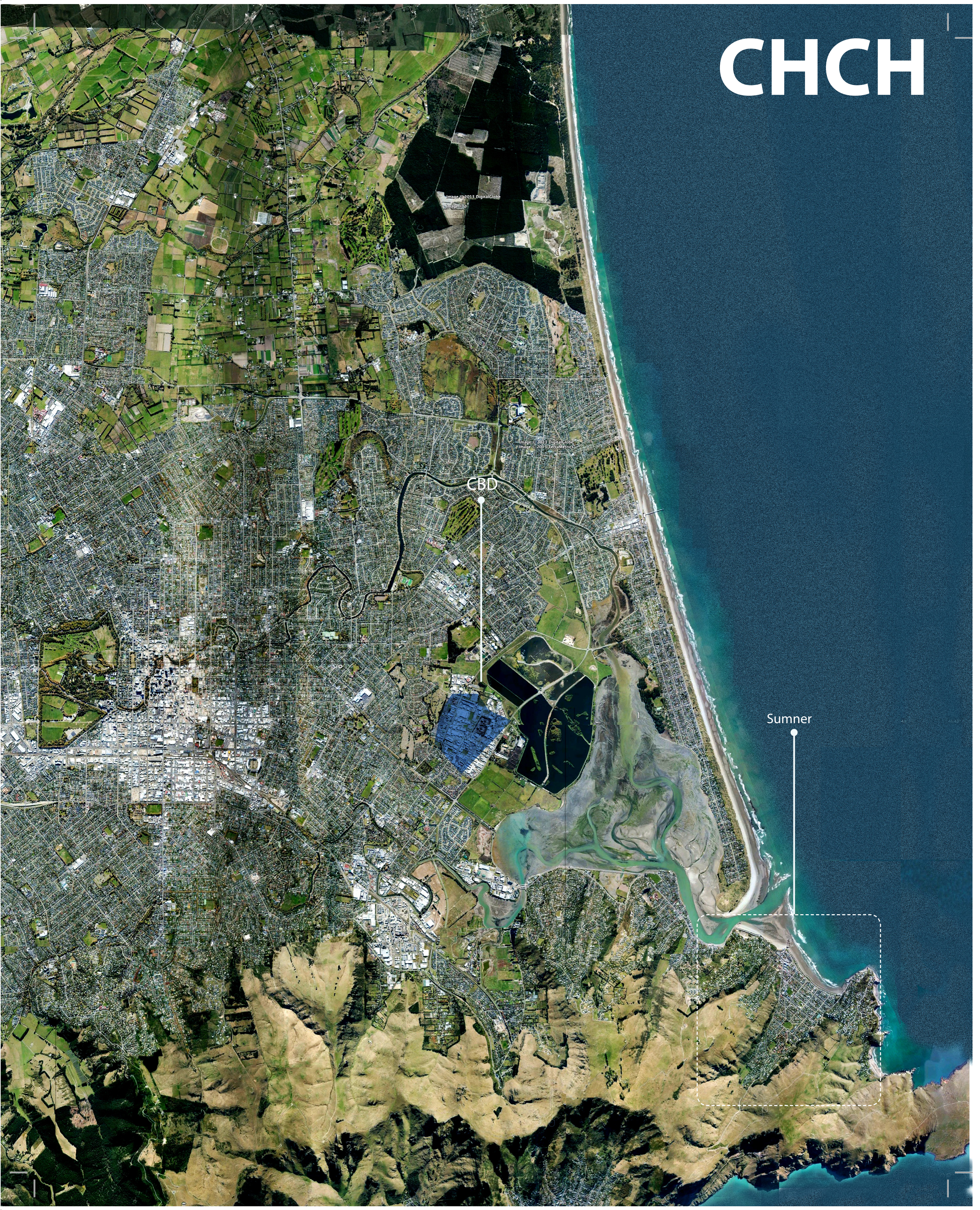




\section{Earthquake as urban catalyst}

Following the 2011 earthquake, Christchurch's urban configuration has naturally tended towards a polycentric state. Though this multi-centred configuration was, in many ways, already latent within the city's dispersed and suburb-intensive structure prior to the earthquake, the inoperability of the CBD (on account of the significant damage it incurred ${ }^{1}$ ) consolidated this condition as a matter of urgency. While the monocentric configuration of Christchurch was fundamentally brought into question by an absent centre, a less-hierarchical, decentralized urban structure was inevitably brought into play: indeed, the survival of the city depended on it ${ }^{2}$.

Despite efforts since the earthquake having largely focused on the rebuild of the CBD (reflecting, a desire to retain a degree of monocentricity that has spawned debate ${ }^{3}$ ), the reality of a dispersed urban structure has nevertheless taken hold of the wider city in the meantime (figure 13). Indeed, in the two years since the earthquake, the city has been 'taken' to the suburbs ${ }^{4}$, and, given that the CBD rebuild will not near completion for several decades, it seems reasonable to assume that this dispersed urban system has an undeniable stronghold in Christchurch's imminent future: regardless of where urban visions, council efforts, or public sentiment are directed.

1 In 2012 it was recorded that around 1200 building were lost from the CBD (Hoare \& Webby, 2012, p. 31) 2 lbid.

3 Or, indeed criticism (Brook, 2011)

4 (Hoare \& Webby, 2012, p. 31)
Specifically, it is not the decentralisation from the city centre to which this design responds, but the that fact that this dispersal of urbanity by no means correlates to an unstructured system, but rather has catalysed the development of concentrated urban 'clusters' beyond the CBD, in former suburbs (figure 11). These transforming urban spaces are coming to assume a significant role, both in providing an alternative to the city centre (in terms of economics function, employment, for example), and yet maintaining a multi-faceted, integral relationship with other centres in the region (as a cohesive whole). Moreover, with the migration of urban cultures, activities and densities and diversities ${ }^{5}$, to suburban crescents, cul-de-sacs and characteristically low-rise areas, a transformation is occurring in these spaces that is as culturally, socially, conceptually and politically significant as it is morphological or economic. On both counts, the successful transformation of these spaces is critical to the future of the wider city.

\section{One city, multiple hearts}

These emerging sub-centres - and the multifacetted implications arising in the ontological shift from periphery to centre - form the broad cultural, social, political and indeed physical context for this design. Rather than resisting an urban change already underway, this project harnesses the need and opportunity to direct critical attention to these emergent urban spaces that are in no sense peripheral - but indeed, plainly central.

5 Since the earthquake, commercial functions have relocated from the CBD to suburban areas. Where in 2011, 60 per-cent of offices around the wider city intended to relocate to the CBD, in 2012, this number had reduced to 30 per-cent, suggesting a more permanent change. A sim lar trend can be seen for retail outlets, cultural institution (Hoare \&Webby, 2012, p. 31)
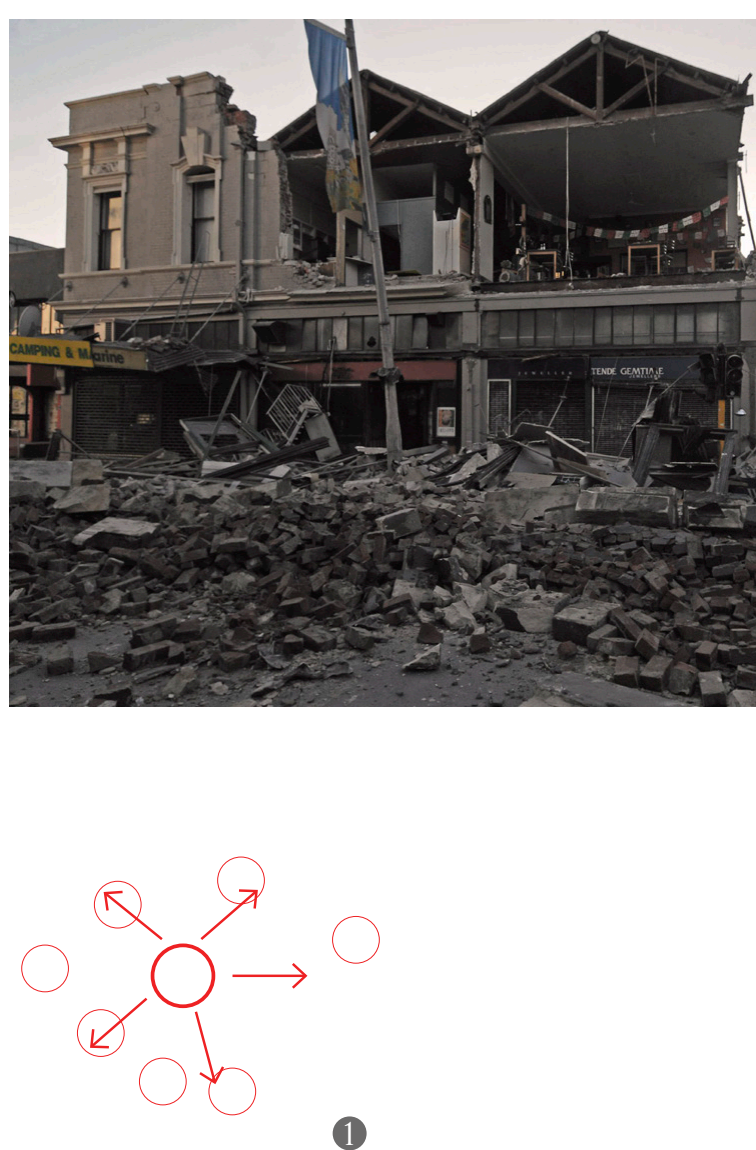

1

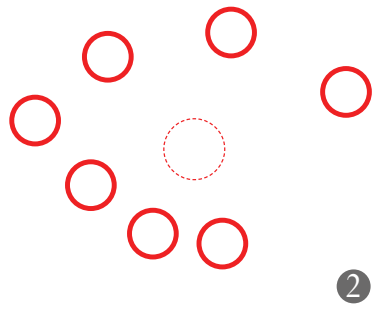

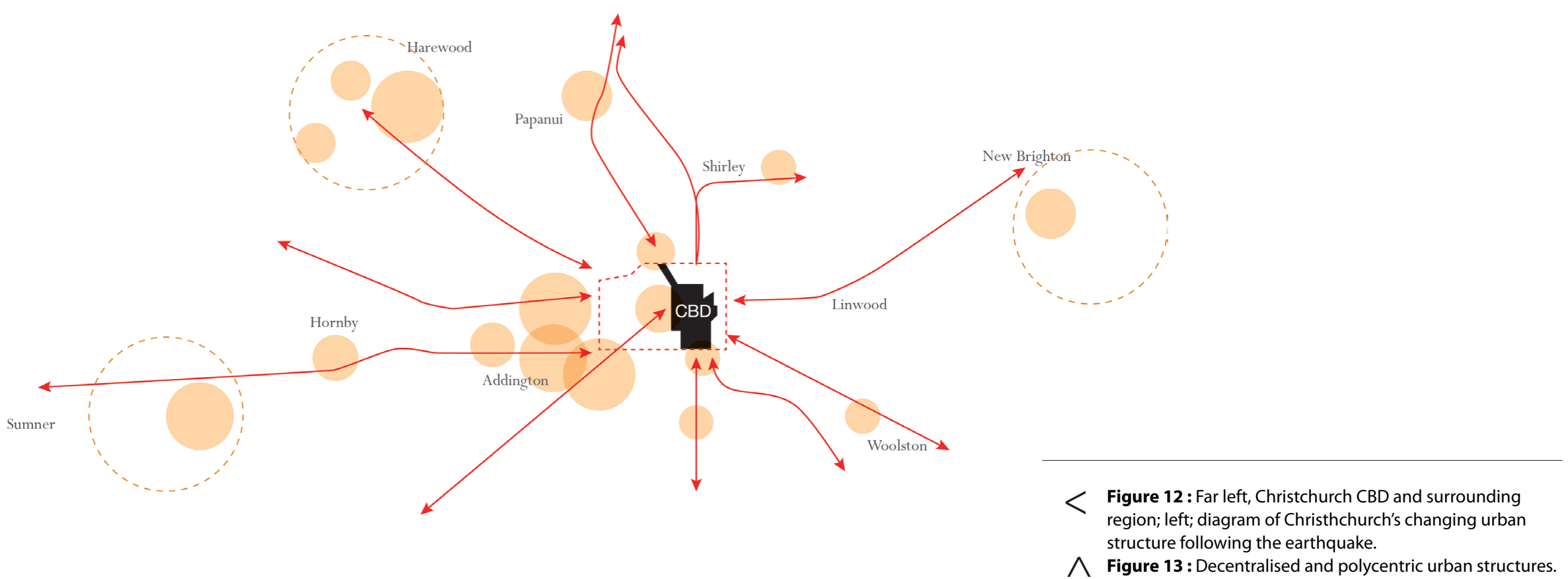

Figure 10 : Above: destructed $C B D$

Figure 11 : Below: decentralization from the city centre (1) can be seen to have corresponded the concentration of peripheral centres (2). 


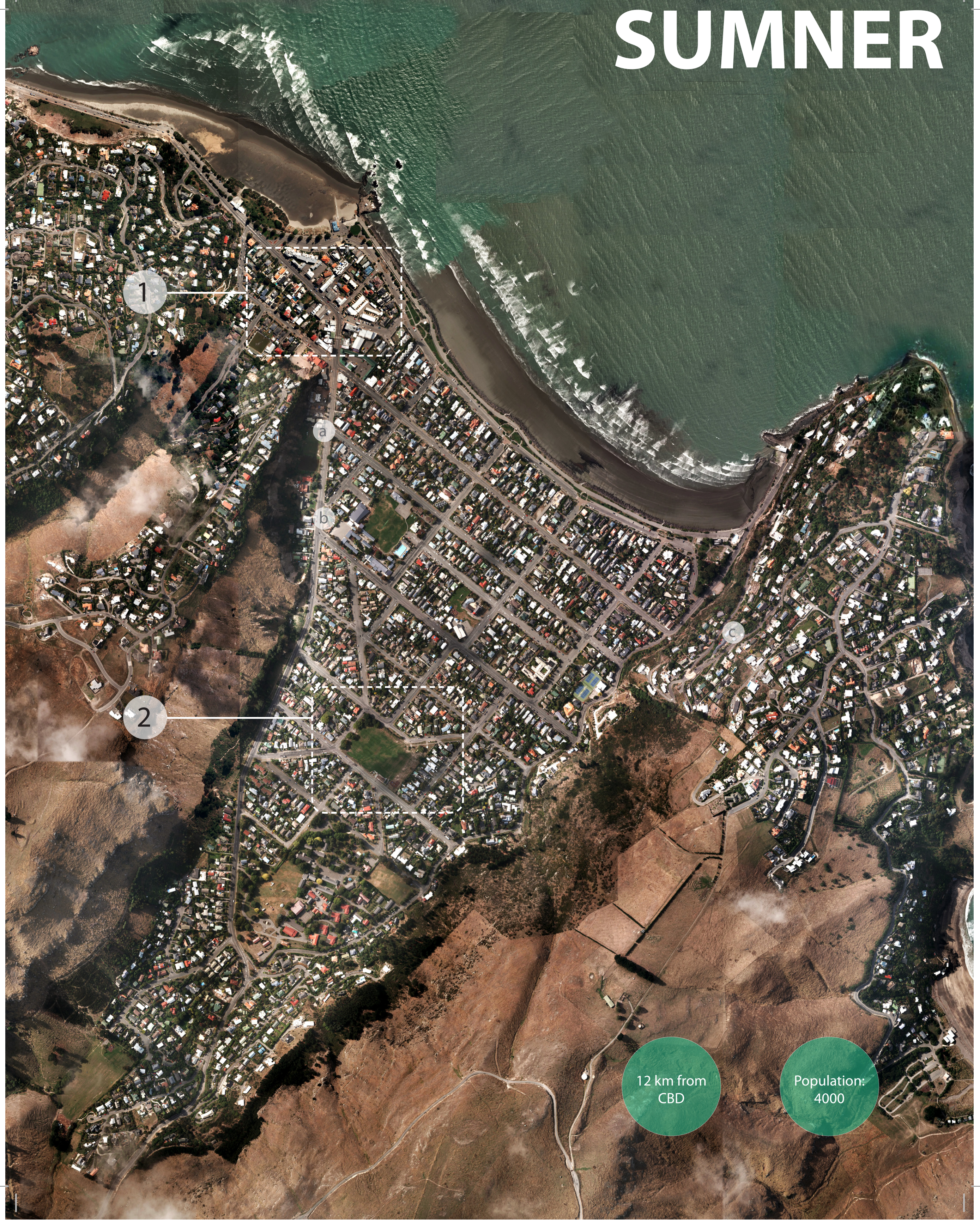


Sumner is one of Christchurch's peripheral suburbs, wedged within a narrow valley twelve kilometres South-east of the CBD, and housing a population of approximately four thousand ${ }^{6}$. This coastallyconstrained and topographically-bound maritime village attests to an endemic New Zealand suburban condition (in terms of its size, aesthetic and largely mono-functional nature); yet, is also duly noted for its strong sense of identity and its charismatic independence within the larger Christchurch region. Today, while the suburb is well-integrated within the wider city, its physical displacement from the CBD and its strong communal vibe enable its relative autonomy, and distinct identity, within the urban region.

\section{A Micro-urban Setting}

At face value, Sumner is as any Christchurch suburb: one of privately-owned, single-family bungalows, $1 / 4$ acre sections, and a quaint low-rise town centre. However, beyond this, Sumner's relationship to the sea permeates its character, and is essential to its sense of place. Indeed, not only is its history dominated by anecdotes of coastal revelry (fig) but today it vehemently upholds this reputation; serving as a coastal escape or weekend destination nestled conveniently within the confines of the city ${ }^{7}$.

Though Sumner incurred significant damage from the earthquake - a substantial part of its town centre destroyed, and many houses

6 (Statistics New Zealand, 2013

7 (Manhire, 2012) sees that Sumner has always attracted visitors on this account. literally left hanging - a substantial community effort emerged to restore the strength of the suburb (figures 17-19). Here, where falling rocks and landslides induced damaged around the edge of the suburb, and to the hill-side residences, the necessity arose to re-focus the recovering suburb inwards - and to consolidate Sumner as a dense, compact centre.

\section{An Uncanny Parity [as proof]}

Indeed, with the likely influx of activity from Christchurch's city-centre, Sumner's geographical constraints indeed lends itself - naturally and necessarily - to densification and intensification and consolidation (over expansion). A tantalising comparison is provided here by Wellington's $\mathrm{CBD}$, whose scale and topographical and coastalconstraints nature bare an uncanny resemblance to Sumner's (fig. 15). Hypothetically ${ }^{8}$, this suggests that Sumner's eventual consolidation as a verifiable dense centre regional centre seems quite possible.

\section{A Dormant Sub-centre}

In this way, although a polycentric discussion has largely focused on Christchurch's Eastern Suburbs ${ }^{9}$, Sumner's post-quake condition, strong sense of identity, as well as its autonomy and physical displacement from the $\mathrm{CBD}$ and uncanny urban parity - suggests its emergence as an urban subcentre may be worth speculating.

\footnotetext{
Insofar as the though the population is significantly les Sumner than Wellington 9 (Hoare \& Webby, 2012)
}
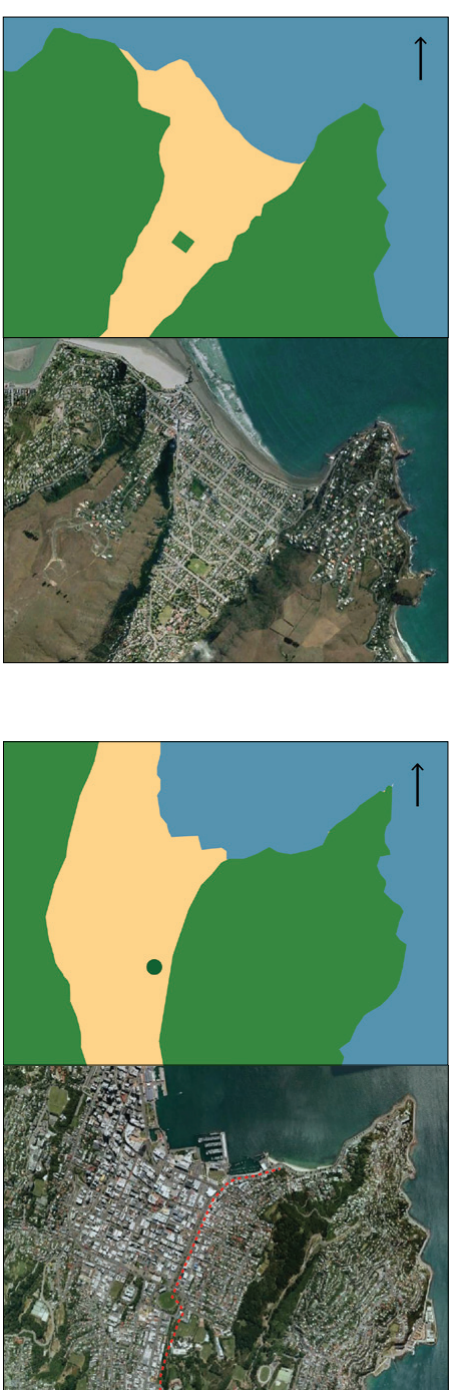

Figure 14 : Above: A group of bathers in Sumner, 1918

Figure 15 : Above: A comparison of Wellington and Sumne to the same scale reveal uncanny resemblences.

$<$ Figure 16: Left: Sumner with existing town centre (1) and chosen site, St Leonard's Square (2)

$\checkmark$ Figure 17: Below left: A main street marred with shipping containers.

Figure 18: Below: Giant bolders and landslides in Sumner. Figure 19: Below: A damaged house hanging off the Sumner hillside.

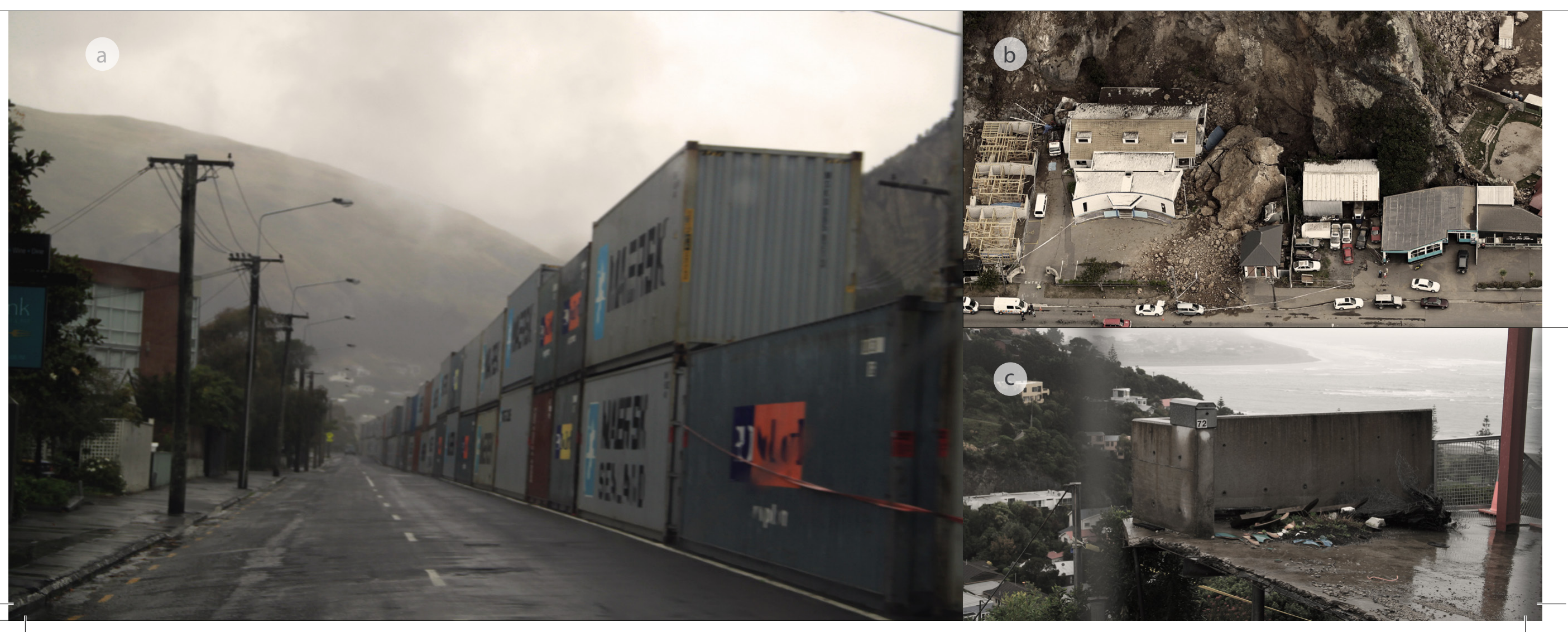




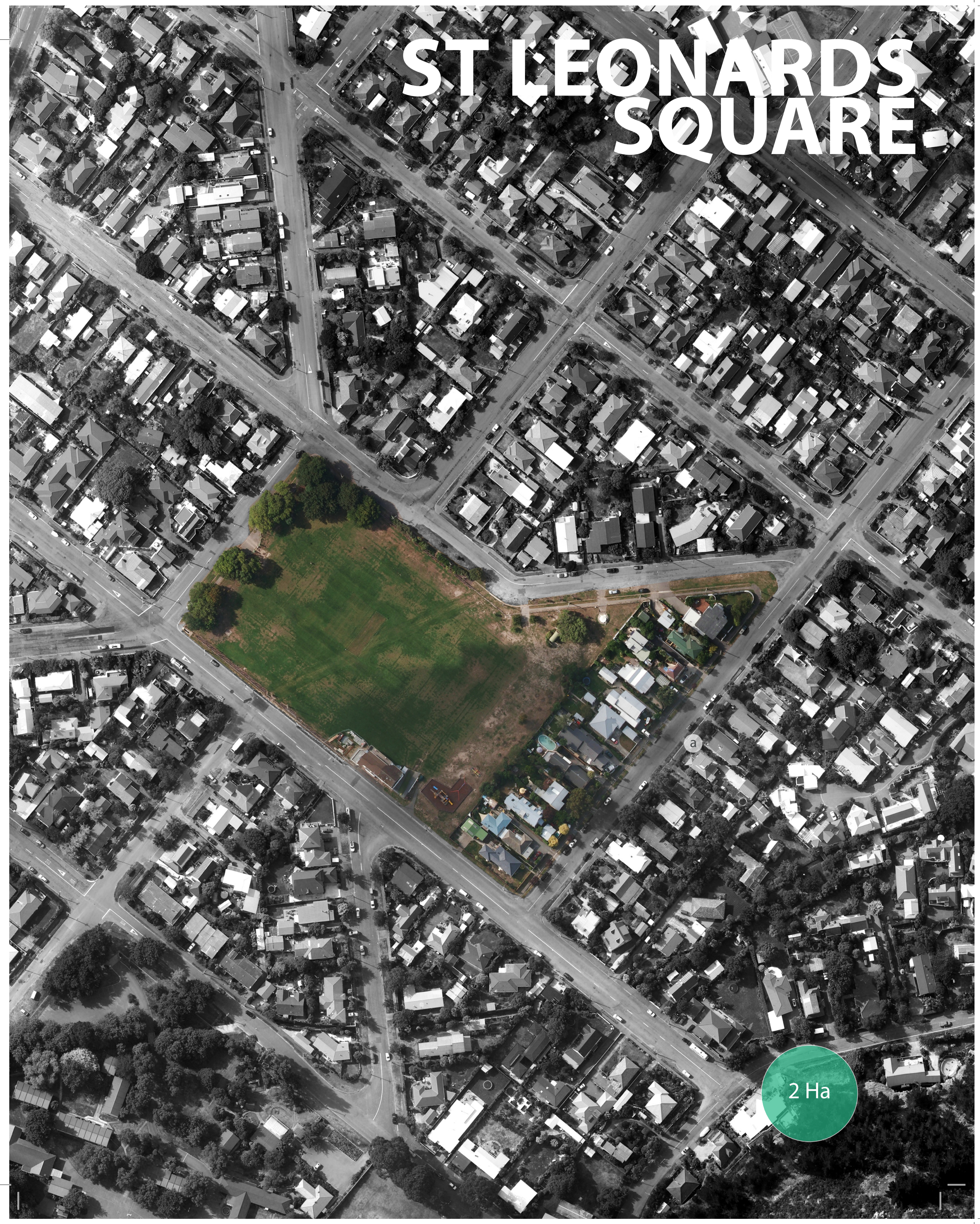




\section{a latent (sub)urban site}

\section{A New (old) Town Square}

As part of a range of efforts to revitalise Sumner in the aftermath of the earthquake, a vocation to relocate the suburb's town centre surfaced. The first option was to restore the original village at the North West end of the suburb; the second was to develop a new town square on an alternative site. (figure 22)

\section{An Unrealised Centre}

The proposed site - St Leonards Square - is located squarely in the middle of the Sumner, an as anomaly arising from two conflicting visions for the suburb during its planning phase (fig. 23). Though, originally configured as town square, this role was never realised: as the frontal subdivision took precedence and the town-centre was developed adjacent to the beach. On this account, today this 'square' operates as a village green and sports field which sits quite apart from the town centre. It is enclosed by residences, and used as a thoroughfare (a permanent diagonal path etched into it) and a Saturday morning sports ground. Aside from the anomalous strip of housing located on the South-east side of the site (Clark Street), St Leonard's Square currently lacks definition and presence (figure 24).

\footnotetext{
Figure 20 : Right: St Leonard's Square in its Suburban context. Figure 21 : Above: A Saturday morning sports field.

Figure 22 : Above: location of St Leonard's Square and existing town village: two prososals for a new centre

Figure 23 : Above: Site diagram indicating two sub-divisions (shown in grey) which meant that this site was never realised as a town-square

Figure 24: Below: (view a) Residential bungalows typical of $V$ the site's immediate context.
}

Without negating its current role as a communal, recreational park - but rather, attesting to its potential as a quintessential suburban space - this site is seen to present a latent nodal opportunity, and one fertile for conversation regarding this intensifying and diversifying suburb. The site's sheer scale as well as its, broader cultural and historical significance suggests it may play an expanded, central role as the urban context undergoes change, especially assuming the infrastructure of the Sumner is unlikely to change. St Leonard's square may be seen to sit not just at the physical but, indeed, socio-cultural intersection between Sumner's past and future conditions. In this way, the site is a latent centre, within a potential (sub) centre - both of whose centrality is incumbent on that of a broader urban context.

\section{A Latent Sub-centre Site}

Though the vocation to re-develop St. Leonards Square as a town square eventually lost favour - and the current village centre is instead being re-instated - the opportunities presented by this site, in the context of an intensifying suburb, are indisputable.
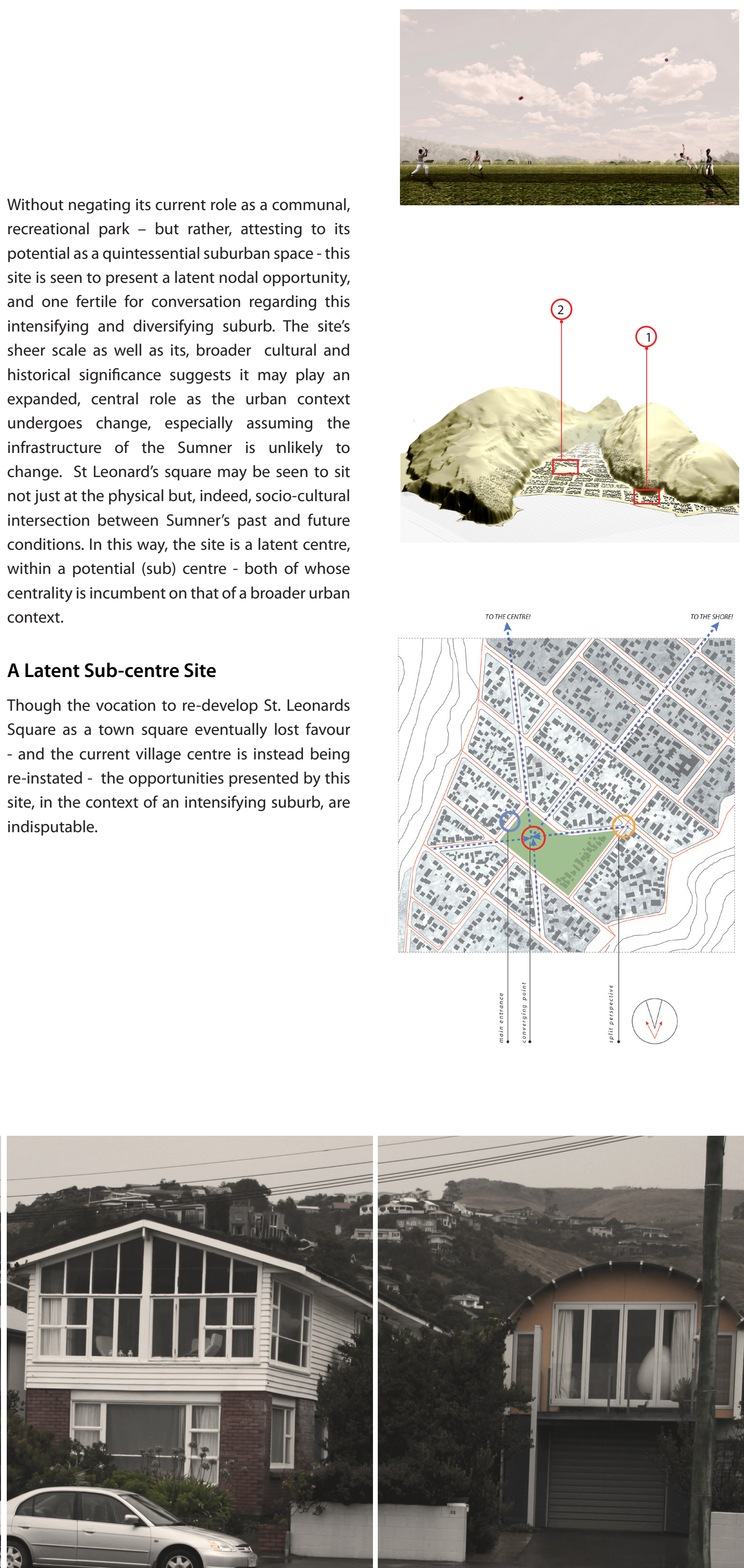
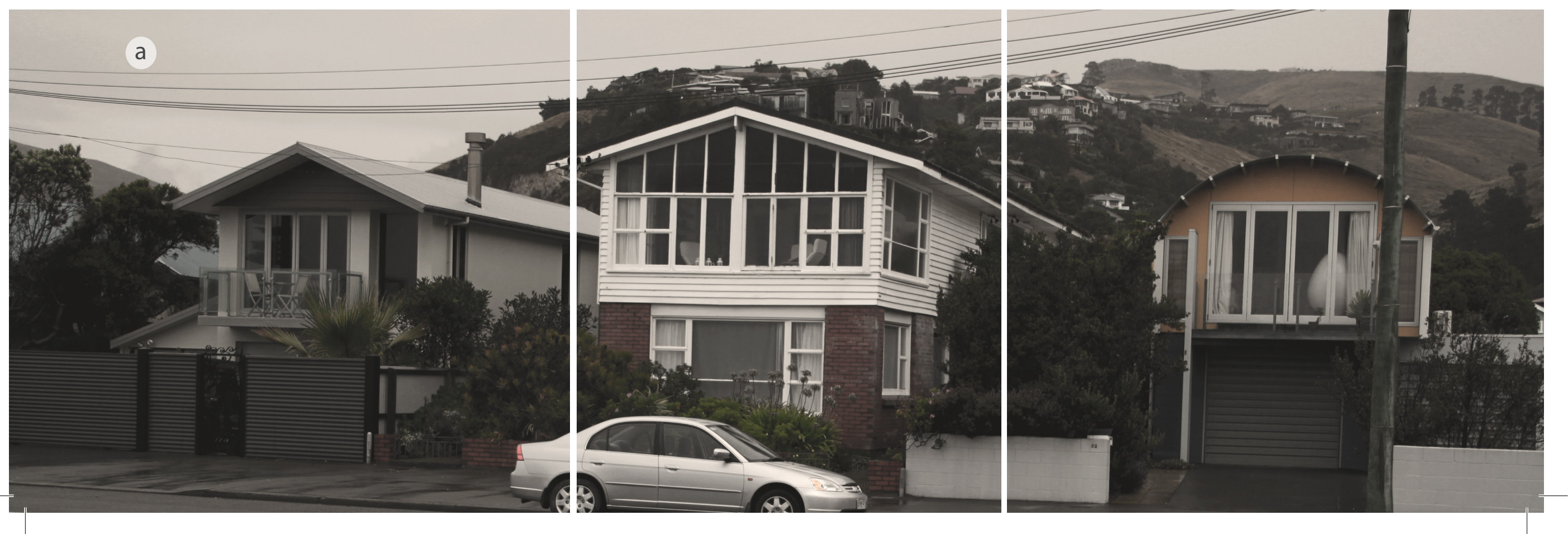


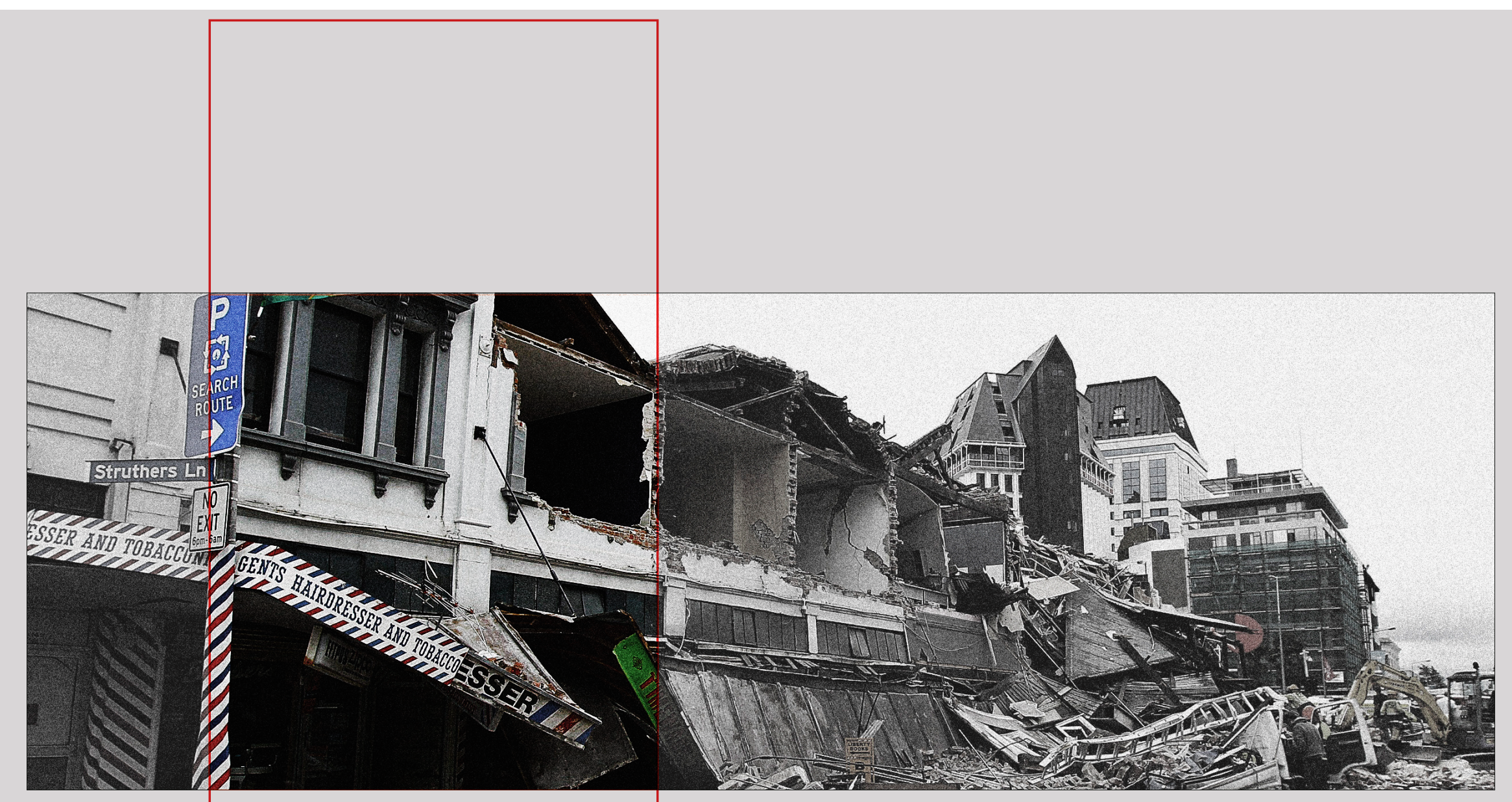

1 a latent city of multiple hearts

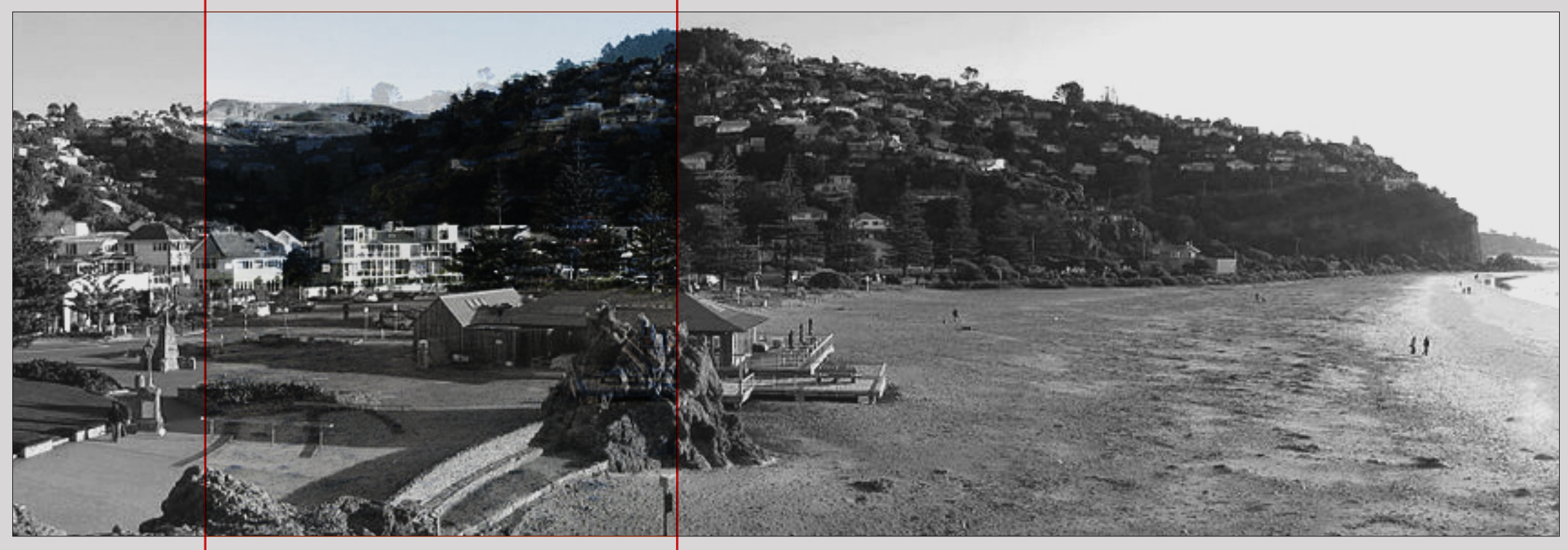

2 a latent sub-centre

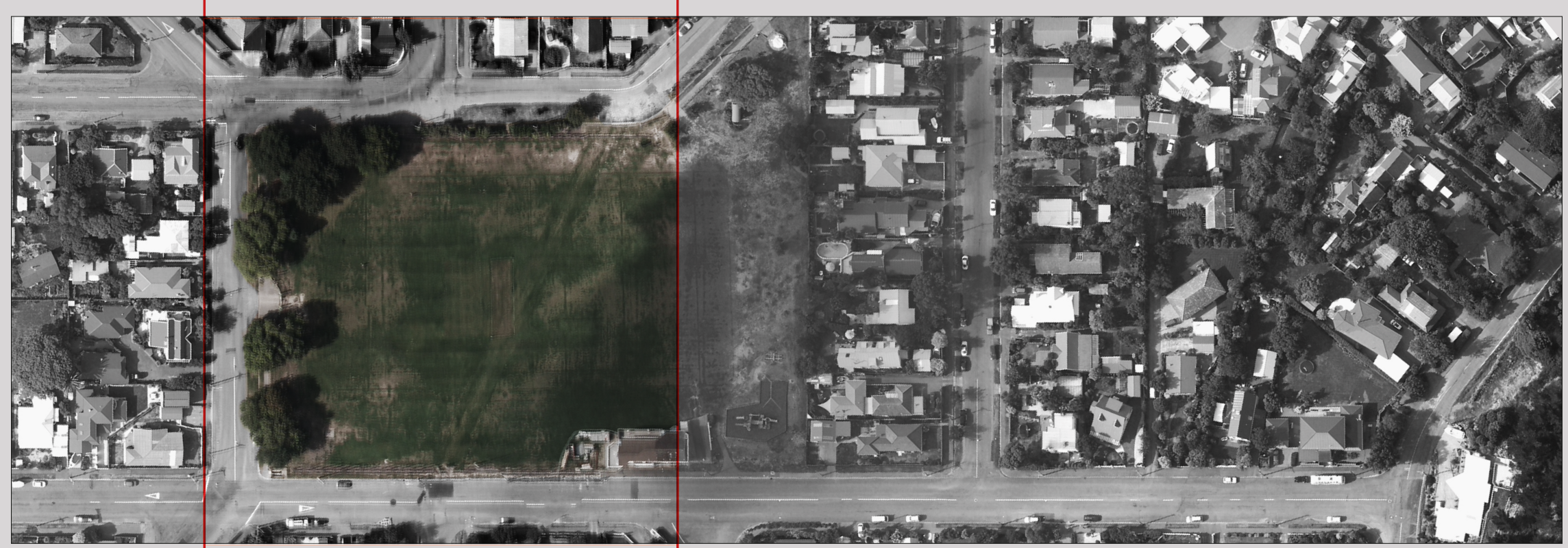

3 a latent (sub)urban site 
A Latent, Threefold Context.

The three conditions described - a city of multiple hearts; an emergent sub-centre and a latent (sub) urban site - form, in equal measure, the multiple context(s) of this design project (figure 25).

As well as being understood each in their latent states, these contexts are seen to be inextricably bound to one another: the likelihood of the second resting firmly on the materialization of the first; and, likewise, the third on the second.

This design-project anticipates (or rather, presumes) that the projected-scenario and contexts of the previous pages will inevitably unfold ${ }^{1}$.

In this sense, the design is fundamentally and intentionally speculative in nature. 


\section{ANAGORA* ANEW}

* n. pl. ag.o.rae or ag.o.ras

1. The centre of athletic, artistic, spiritual and political life of the city ${ }^{1}$

2. A popular political assembly.

3. the place where such an assembly met, originally a marketplace or public square. ${ }^{2}$

This project proposes to re-appropriate Sumner's former village green as a cultural, political, social and athletic centre. The design harnesses and exploits the spatial, formal and programmatic opportunities of an emergent sub-centre condition, to deliver St Leonard's Square with a new densified, diversified, and rarefied character - and presence - within its immediate and wider context. In so doing, the design not only attests to, but enacts, the possibilities of this urban reality at multiple scales - and implants them firmly within this latent Sumner site. Both as an urban and architectural gesture, this radically-contextual live-work Agora serves as catalyst for Sumner's imminent transformation from suburb to sub-centre. It asks: what can architecture do in the emergent sub-centre? 
4

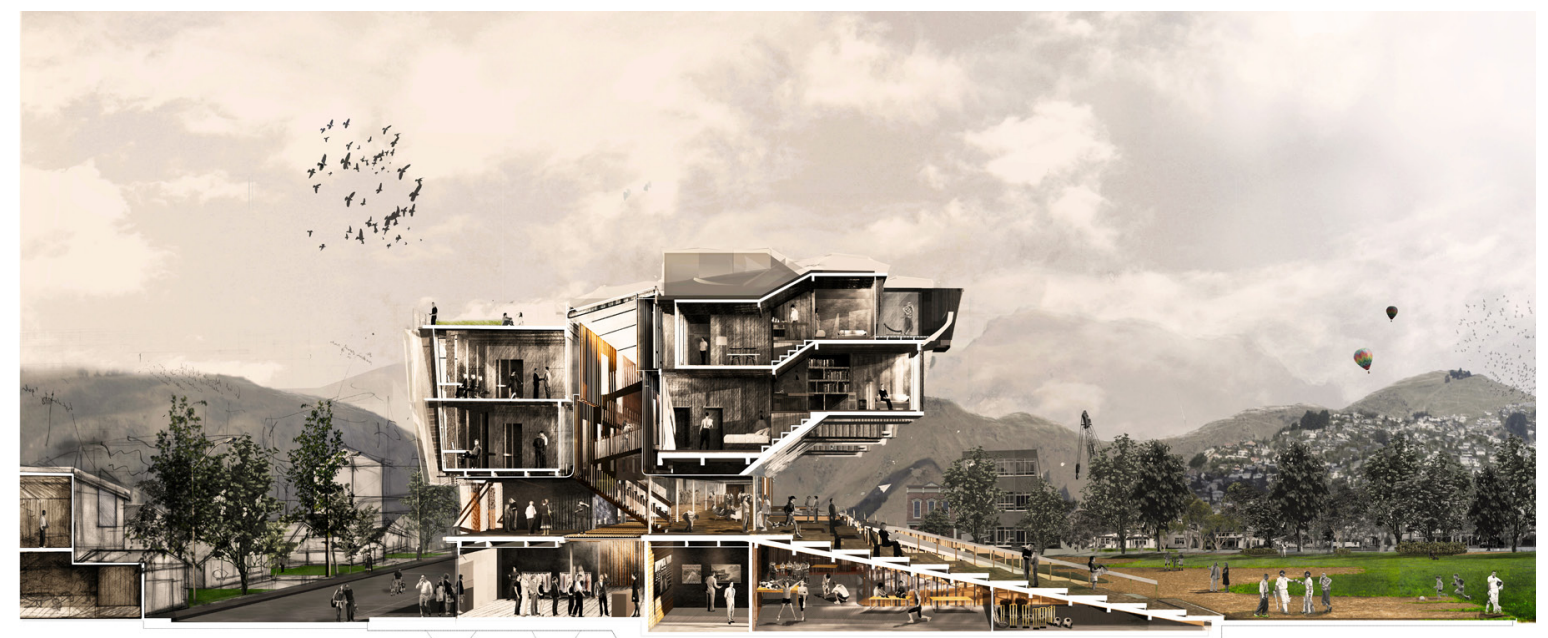

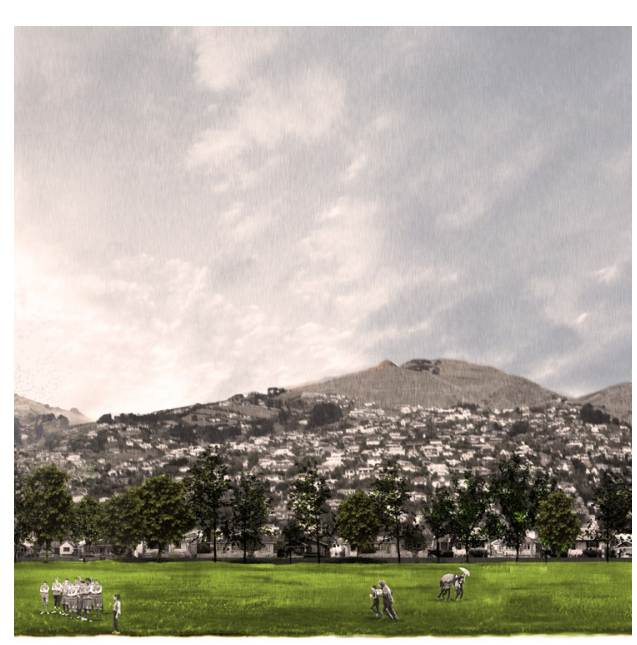

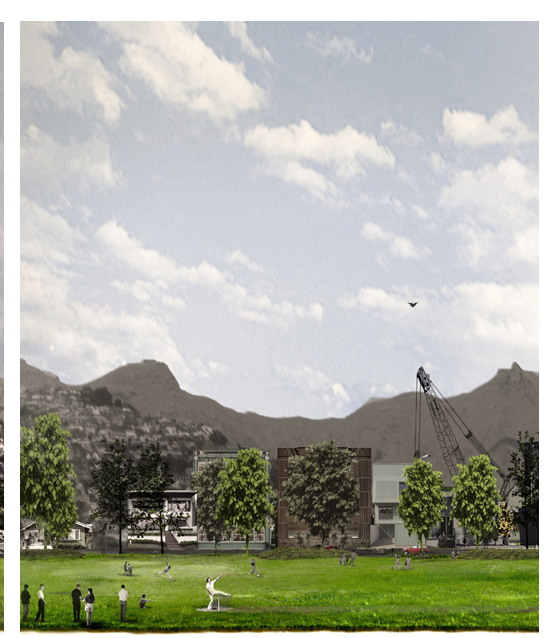



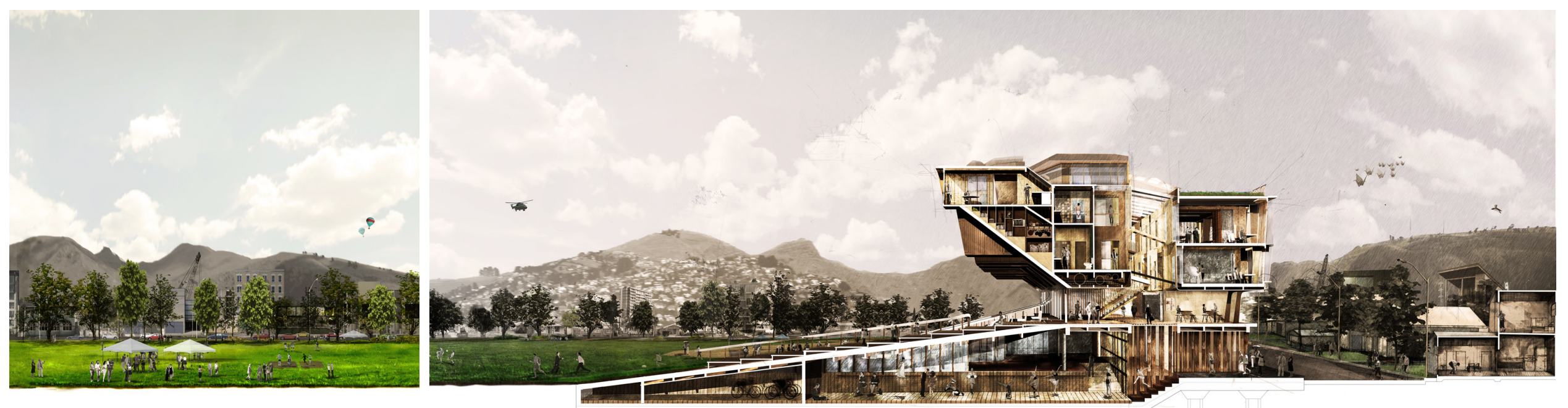

$<$ Figure 26: Longitudinal section 


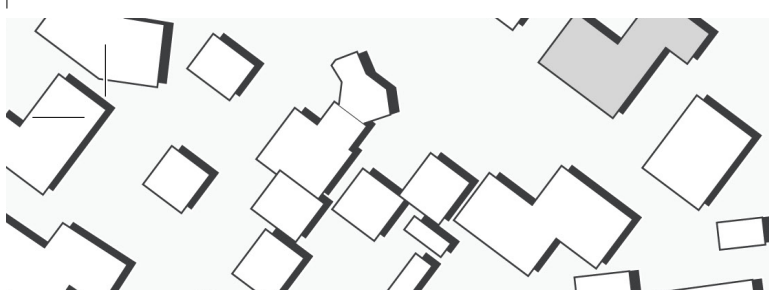
4

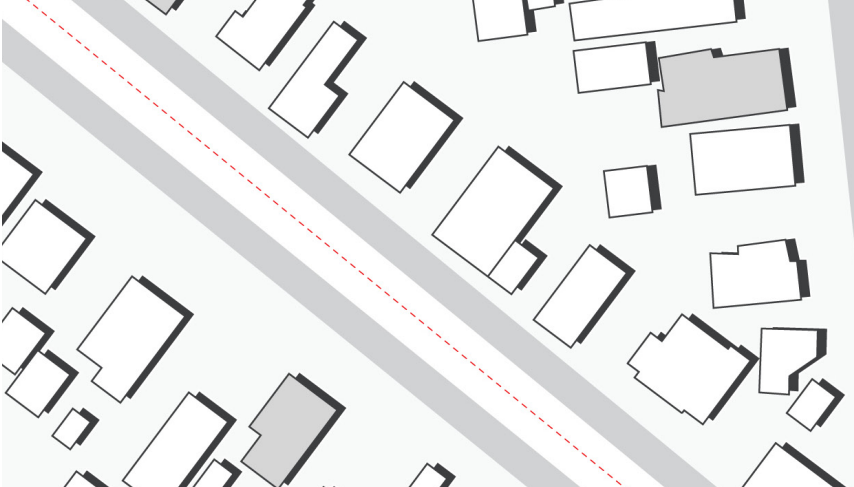

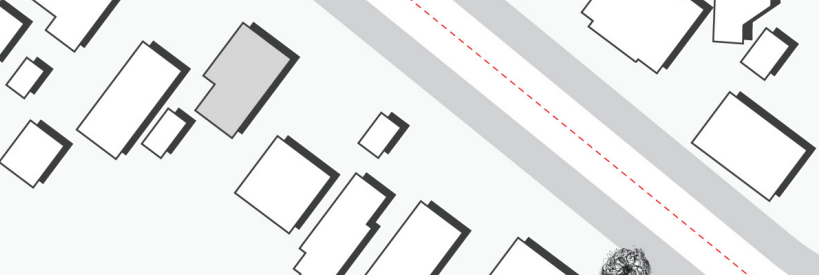

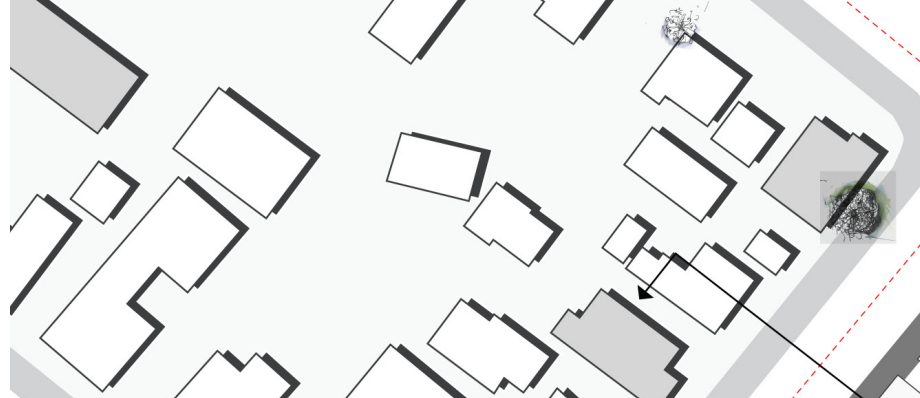
$3 \quad>3$ \rangle$^{>}$illage green $>$

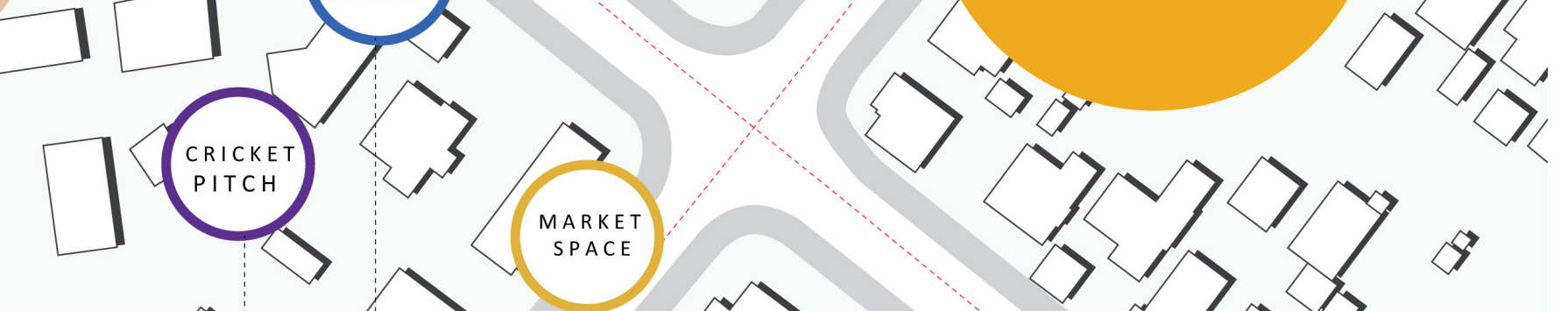

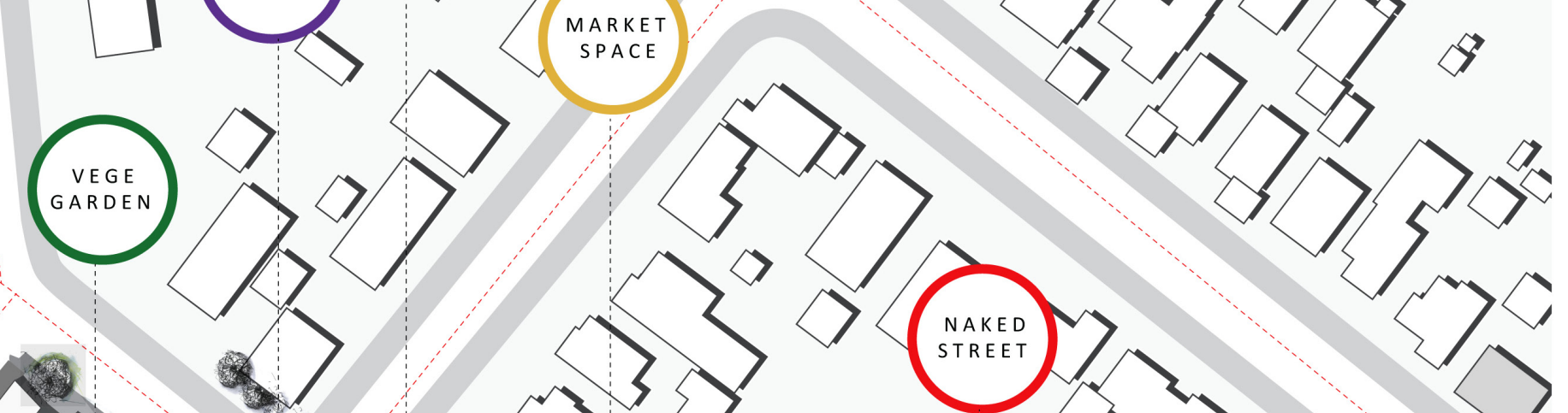

i,

$\prod_{\square \rightarrow 3}^{2}$

$3,2>23$

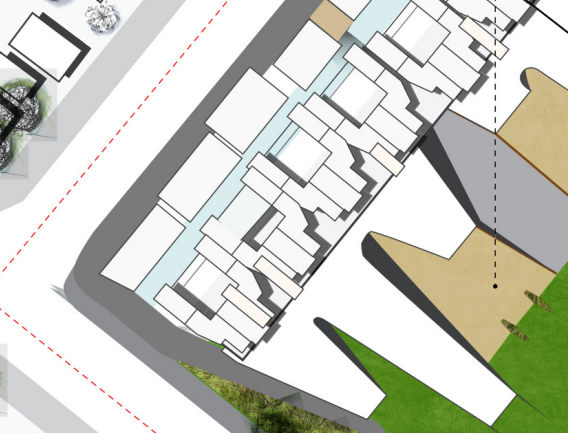

(2)

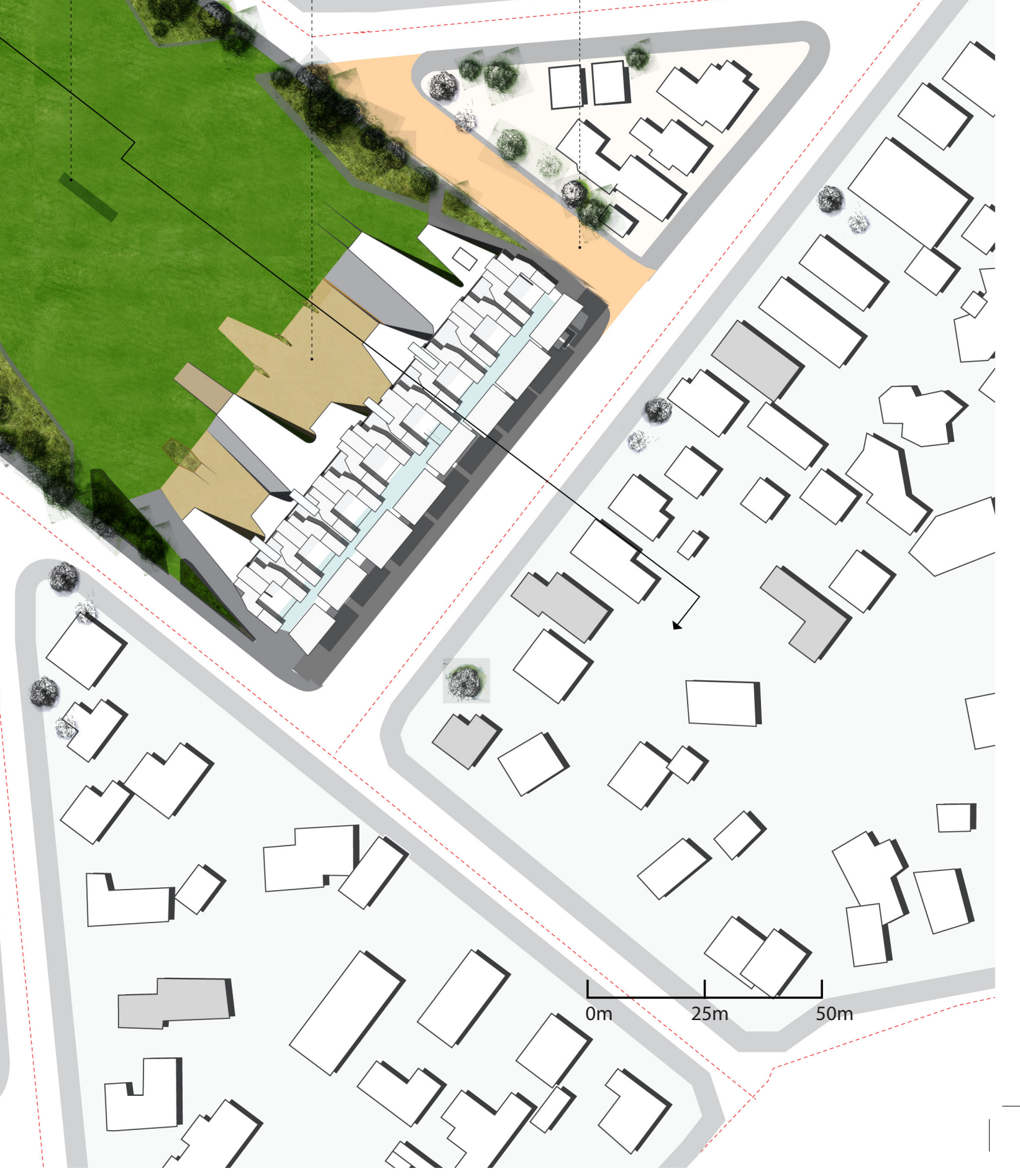




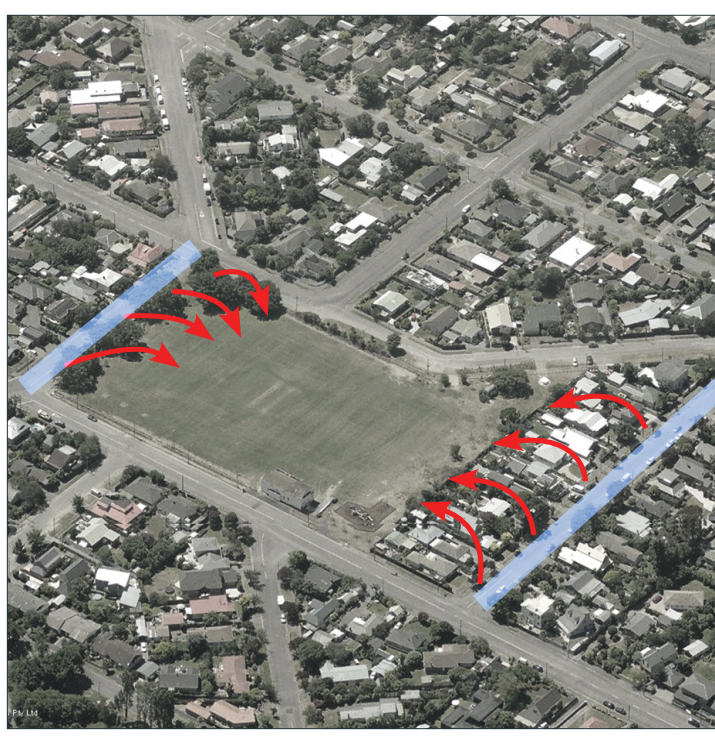

Figure 27 : the distribution of built form directs focus simultaneously onto the open space (as centre), and on the two bordering streets (as active urban edges).

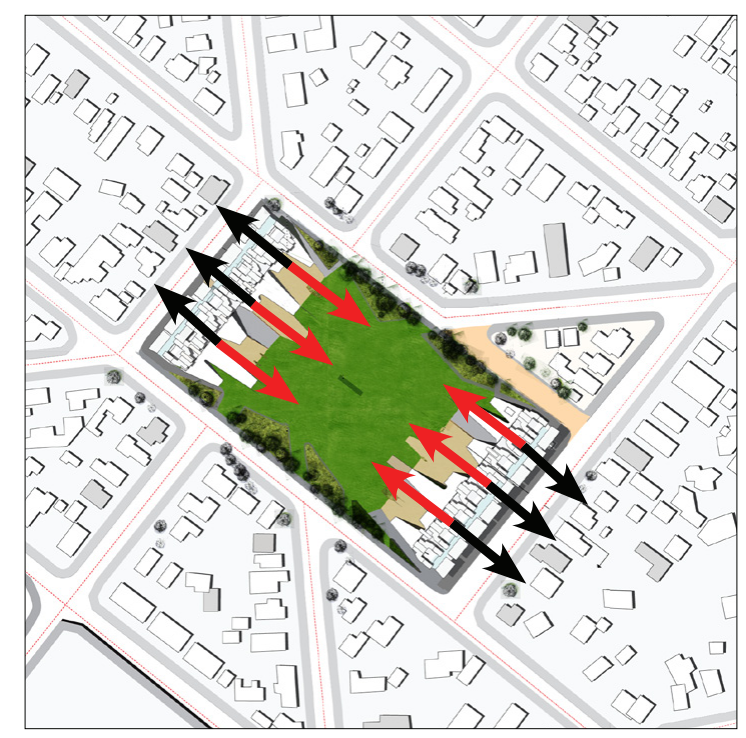

Figure 28 : the strips of built form operate as a two-sided gesture, addressing both the central space, and the street context (understood to have no 'front' or 'back')

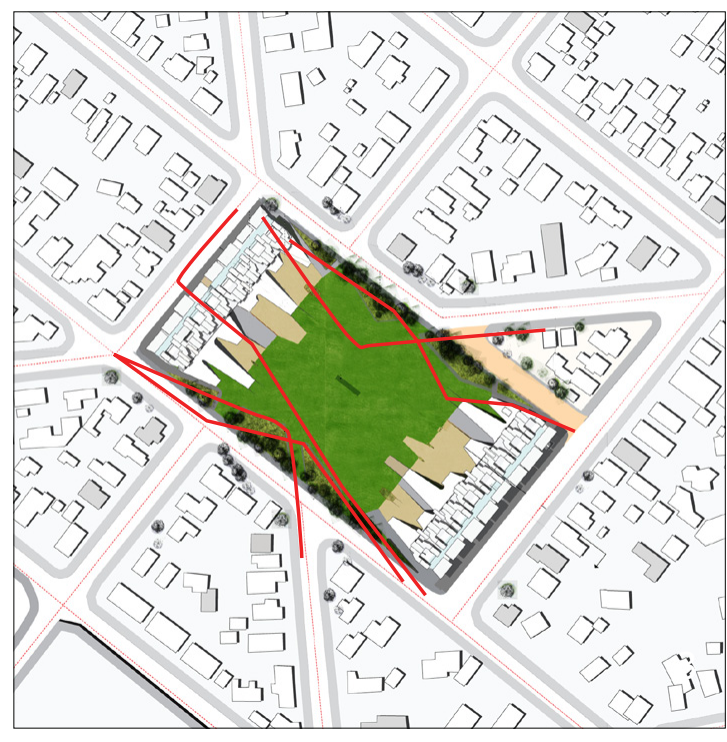

Figure 29 : porosity and permeability is facilitated by the figure-ground condition. On the North-eastern side of the site, a naked street allow easy, public access and allow further integration with street network.
This design re-calibrates St Leonard's Square as a rarefied public-space, straddled by elongated strips of built form on two sides and left glaringly open to the suburb on the others. Where previously the boundary of the green-space was a nonchalant urban affair - demarcated merely by a fence and a footpath - here, the distribution of the built along permits two basic urban operations. Firstly, it refocuses the 'square' as a centre and reintegrates it into the surrounding street network; secondly, it articulates the surrounding streets as clear, hard, active edges, such as to re-work them as latent urban (public) space - rather than the mere backsides of the park (fig 27).

\section{Built form as urban medium}

The opposing strips of built form fundamentally uphold this double urban gesture: with no explicit 'front' or 'back' per se, they operate as a two-faced affair, engaging both street (as edge) and the park (as centre) simultaneously; and with differing address (fig 28, 30).

\section{Pavilions, to the park}

Pointing (or poking) inwards onto the central space, the built form bleeds into ground plane, carving out a rich, textured variety of public enclaves through an open-ended, informal and organic interlocking with the ground. An alternating system of terraces and public courtyards softens the boundary between built and open space, and giving new form to the central space, as a continuously-varied, innocuous edge.

While these inward-facing forms evoke a curiously-traditional public space, echoing the originally-intended function of the site (a town square), the park's existing recreational character is retained: both with the clear directionality of the forms, and the openness of the site on two sites. In this way, these two opposing strips of built form impart a state of centrality to the space between them, that caters for equally athletic, public, cultural and social activity: an Agora of sub-centre affairs.

\section{Fabric, to the street}

Facing outwards, engaging the street, the built form is a modulated, staggered street fabric: a edge articulation that both echoes the divisions existing grain of the suburb (assuming similar divisions); yet, attests to a more-urban condition through its density, proximity, and contiguity.

With this two-fold gesture, these elongated, pavilions attenuate the edge of the site: a divisive medium between two proximate, yet distinct, suburban conditions.

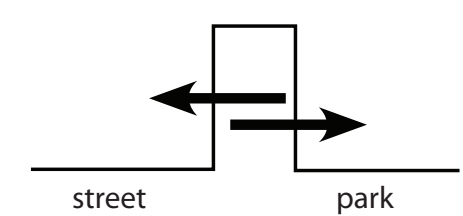

Figure 30 : The basic street-park condition that the built form critically addresses.

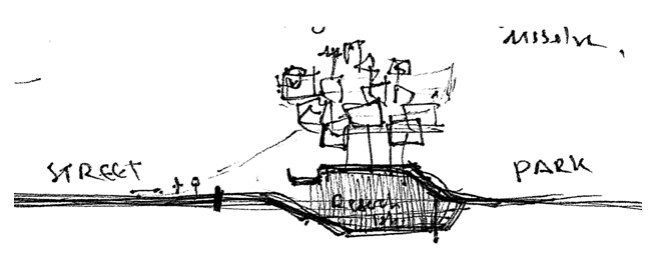

Figure 31 : An early design concept envisaging the built form as a mass operating between park and street. 
The negotiation between the central open space and the street permeates the built form, at the ground plane, and above, invoking a formallyexpressive, densified boundary.

As they occupy the edge, the pavilions peer out and preside over the open space, while presenting a vertical stacking of spaces to the street: a porous yet unequivocal edge condition. On one side, the sloping terraces meet with the ground plane seamlessly, allowing the open space of park to enters up and into the building, where it is compressed between the lower and upper floors as an elevated ground plane. Within, it becomes a central, semi-interior public space that stretches out across the length of the building as a pubic porch; passing-through to the street at intermittent points, and piercing upwards as a central, internal chasm in the $\%$ middle of the building.

Around this void, a series of smaller, moredefined internal spaces are configured: a series of horizontal 'layers' that step-up or step-down as they transition between street and park (a).

Another reading of the building is offered as this compartmentalised, layered, interiorisation of the sub-centre is effectively voided out by the central chasm. Here, the built form is understood as three primary masses that are both connected and separated by the intermediary space: forming an flickering hierarchy between solid and void (b, c).

$$
a^{4}+4
$$

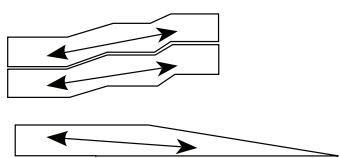

(a)

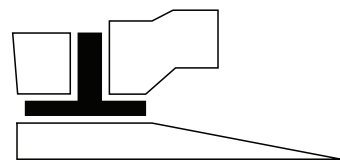

(b)
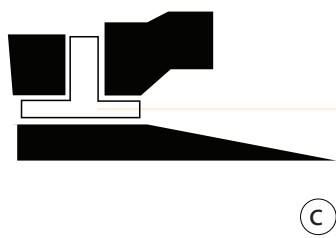

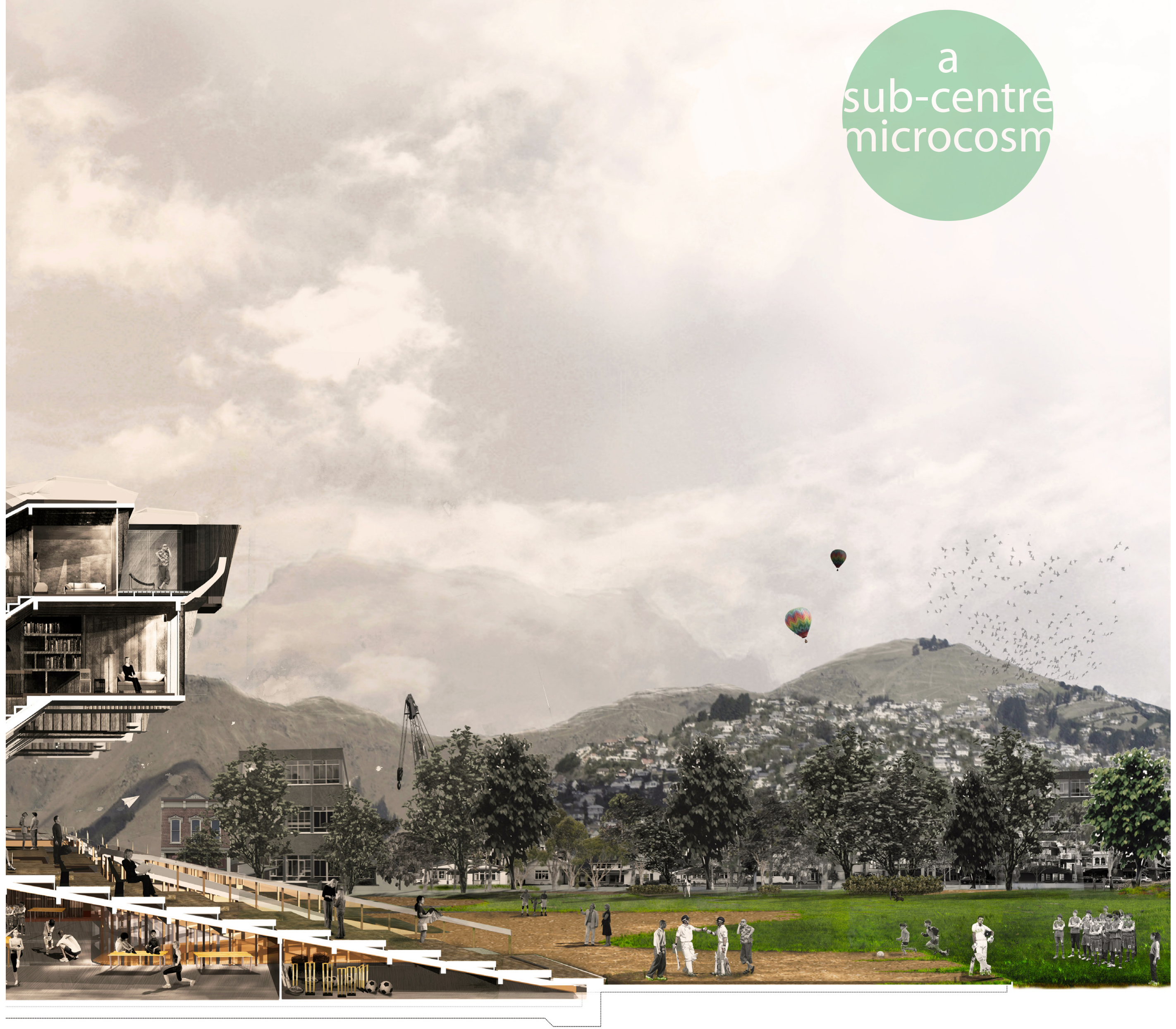

$\bigwedge$ Figure 33: Section through Eastern pavilion. Firmly occupying the edge of the re-calibrated village green, the pavilion negotiates the park and street contexts formally, spatially, and programmtically. Here, the open space of the park enters into the building, articulated as an internal, void that moves across and up through the architecture.

$<$ Figure 34 : Diagrams of the bulding's underlying formal/ spatial logic: horizontal layering; masses shaping - and shaped by - a central void. 


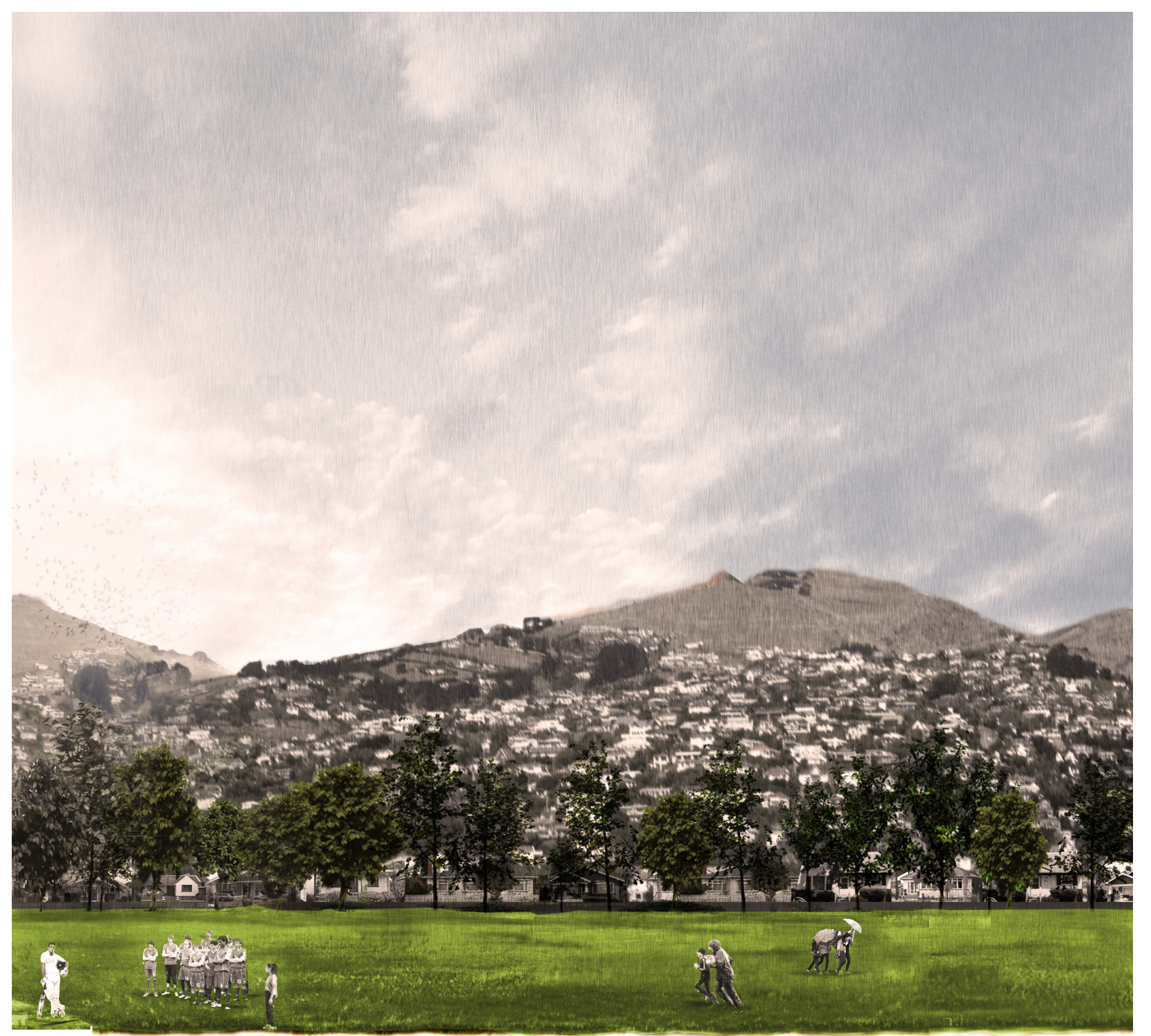

8am Monday, June

A public thoroughfare

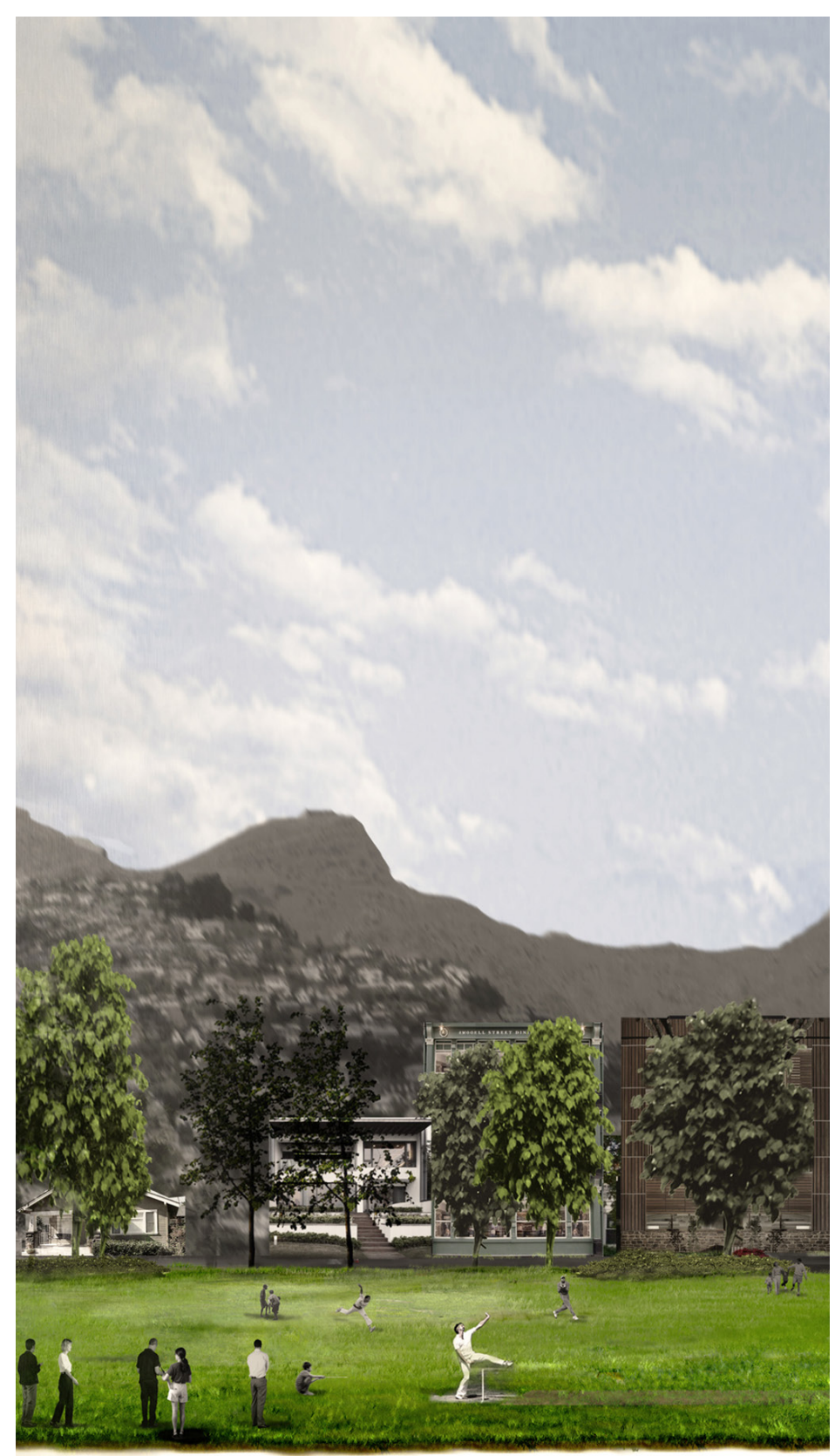

2pm Saturday, Septe A local cricket match 


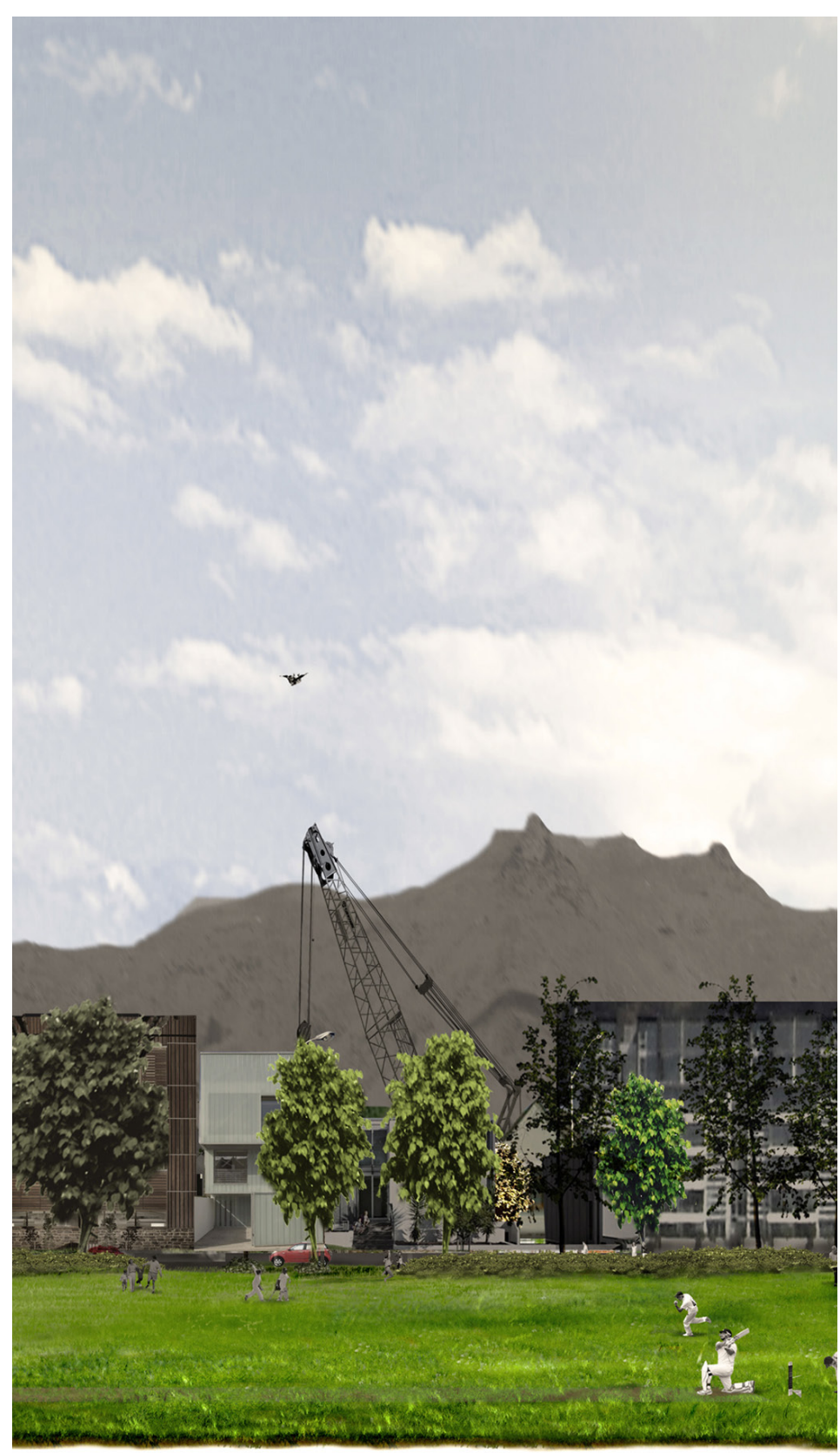

ptember

atch

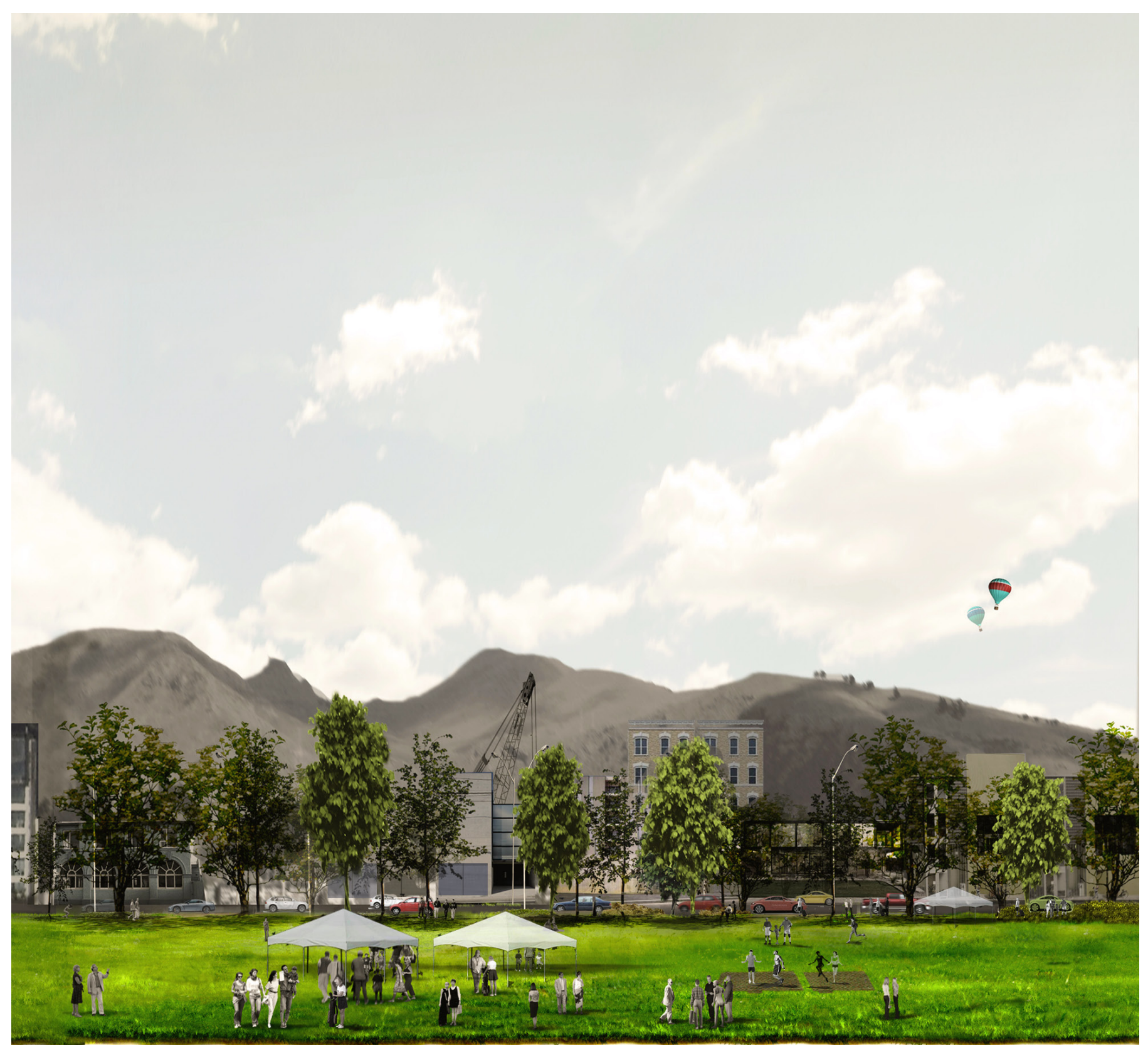

10am Tuesday, December

A public market

Figure 35 : Section through site (continued overleaf): St Leonard's Square as a rarefied public space which retains the parks original recreational function, yet introduces the possibility of further public, social and cultural activity through two densifed edges (an array of activity) and the site's enhanced presence in its immediate and wider context. The background suggests an urbanizing context, from left to right. 
An edge condition embodied

Through their formal and spatial configuration, the pavilions provoke an urban, external and internal dialogue between park and street. They do not merely occupy, but formalize, spatialize and enact the border condition of the site: a densfiied mediating edge that both juxtaposes -and reinforces - the open space it affronts.

As it negotiates this boundary, this two-faced architectural gesture starts to play on the particularities of the context at multiple levels and scales (and certainly, beyond the physical). The built form induces a flickering: not only between park-and-street, but simultaneously, between urban and 'suburban', dense and rarefied; public and private, built and open; present and future.

In so doing, the architecture (pavilions, fabrics or otherwise) operates as a medium: giving rise to dialogues not only between two physical conditions; but between a series of broader cultural, political, social, and programmatic implications that are slung between suburb and sub-centre.

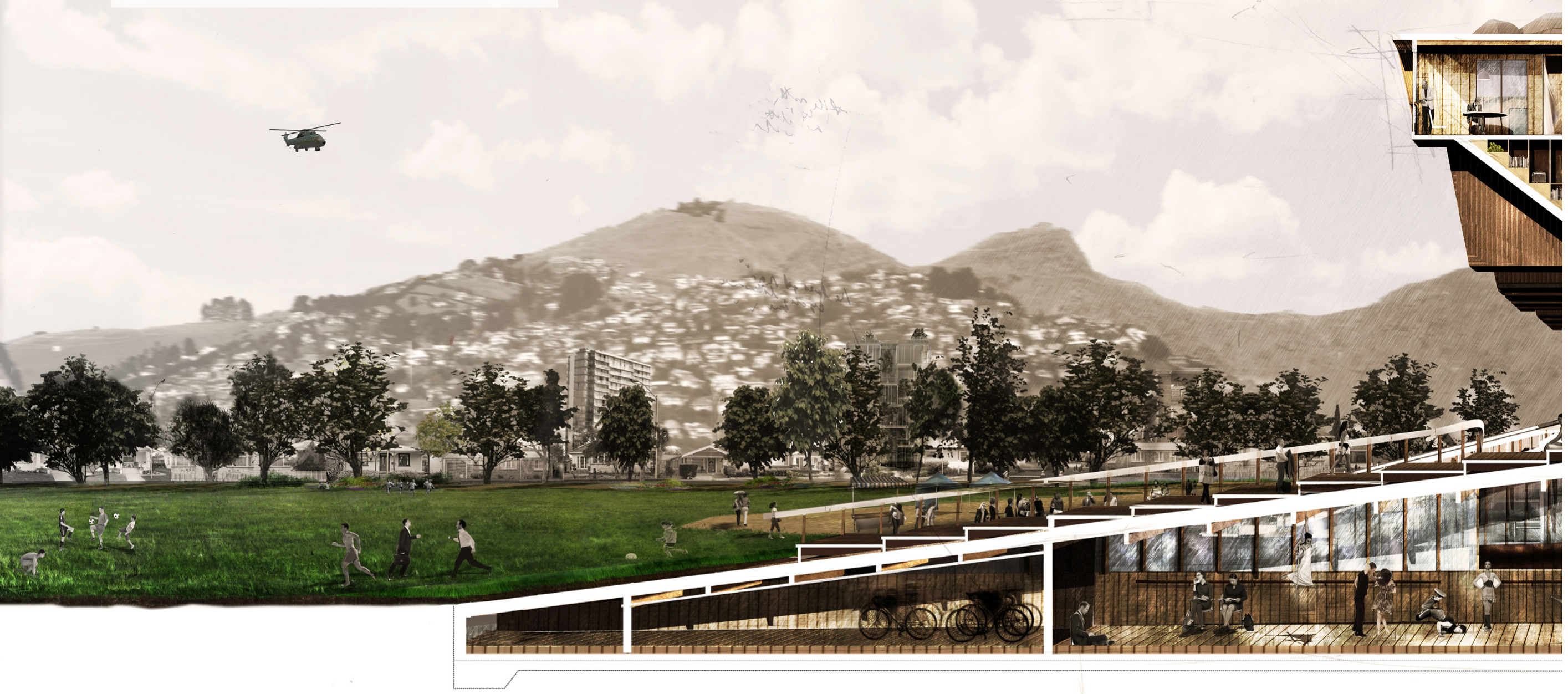

$\wedge$ Figure 36: Above: Section through Western pavilion: The formal and spatial negotiation between park and street allows the architecture to enact the boundary condition of the site. At the ground level, a continous space transitions between street and park: dipping down into a gaping interior that tucks under the grass, as a public terrace becomes its roof.

$>\quad$ Figure 37 : Right: a diagram showing basic flow between park street. 


\section{an
embodied
threshold}

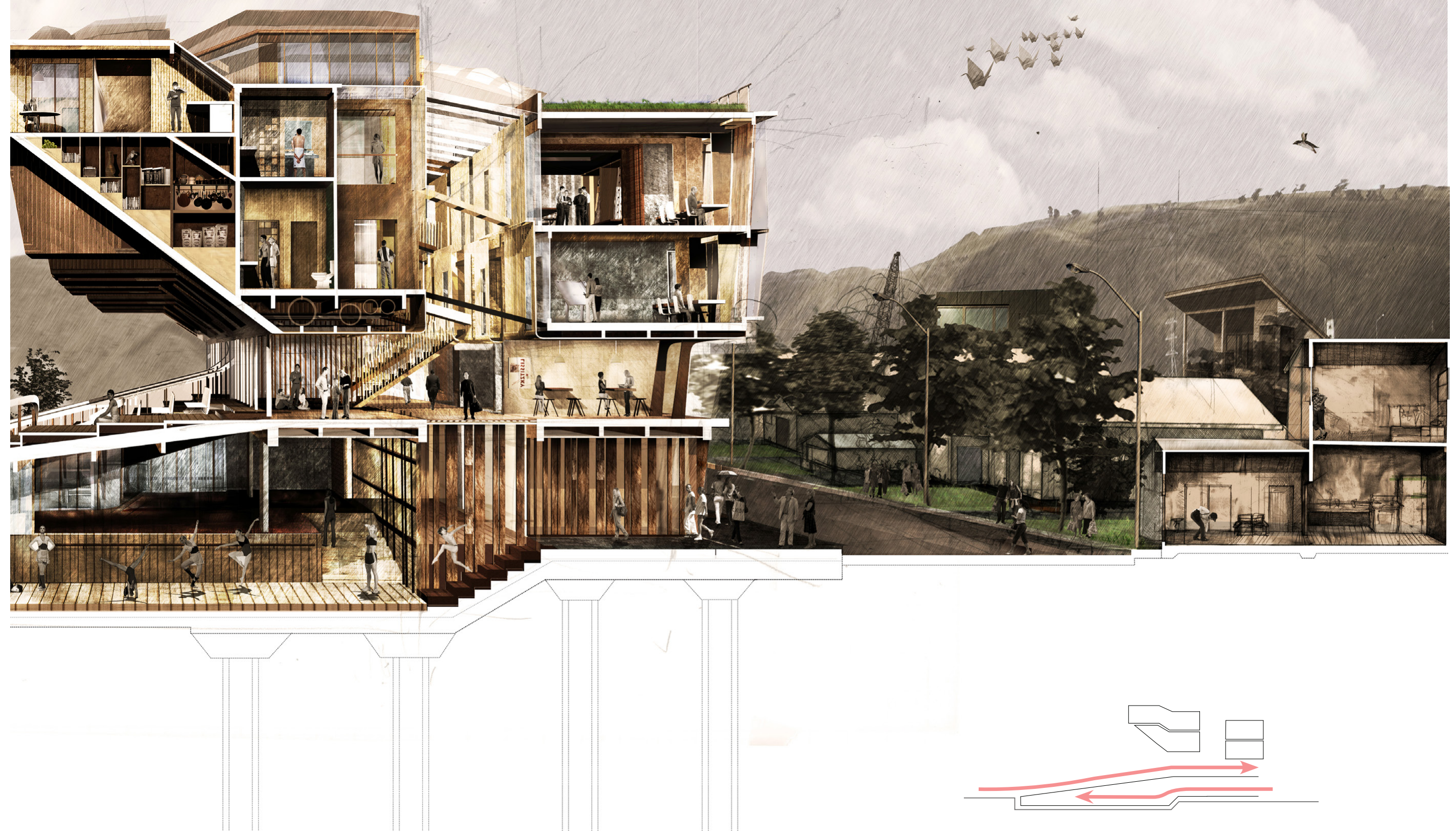


The two pavilions comprise a mixture of living, working, private, public, leisure, retail, commercial and unprogrammed spaces: brought into close proximity across five levels, as dense interiorisation of the sub-centre

Through the underlying two-faced gesture, a variety of spatial types and conditions are created along the length of the building. These cater for the diverse mixture of programmes, which are brought into overlap, intersection and collision by the informal, irregular and organic geometries.

At the scale of each individual building, a system of organisation of spaces is provided by the irregular divisions, derived from the street (see figure --) These offer a basic system of repetition which governs, approximately, the main circulation spaces and points of egress, for example, as well and interfaces with the street and park (essentially staggering and terraces, respectively). (see figure 38).

Within the buildings, these divisions also inform the internal spaces across the five levels, to differing degrees: compartmentalising clusters of street frontage on the ground floor; separating the housing and offices on the upper floors, for example. Notably, at this scale, the spaces are more freely and richly manipulated by the informal geometries of the building: intentionally breaking down the repetitive character that is apparent at the larger scale.
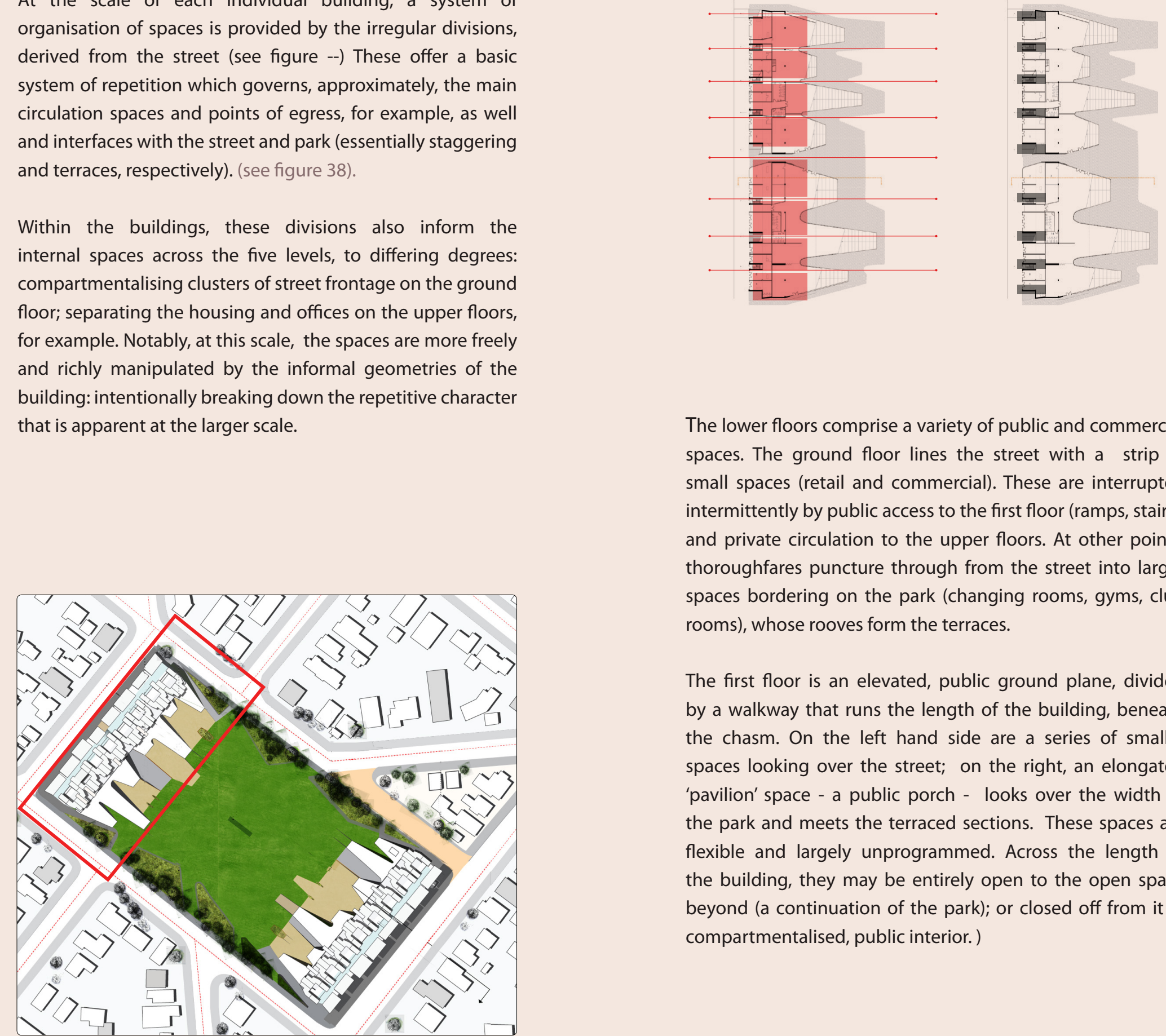

The lower floors comprise a variety of public and commercial spaces. The ground floor lines the street with a strip of small spaces (retail and commercial). These are interrupted intermittently by public access to the first floor (ramps, stairs); and private circulation to the upper floors. At other points, thoroughfares puncture through from the street into larger spaces bordering on the park (changing rooms, gyms, club rooms), whose rooves form the terraces.

The first floor is an elevated, public ground plane, divided by a walkway that runs the length of the building, beneath the chasm. On the left hand side are a series of smaller spaces looking over the street; on the right, an elongated 'pavilion' space - a public porch - looks over the width of the park and meets the terraced sections. These spaces are flexible and largely unprogrammed. Across the length of the building, they may be entirely open to the open space beyond (a continuation of the park); or closed off from it (a compartmentalised, public interior. )

Figure 38 : Above diagrams showing the irregular division underlying the organisation of the spaces (left) and the location of circulation point as dictated according to these divisions.

Figures 39 : Right: Ground floor plan Figures 40 : Right: First floor plan. 

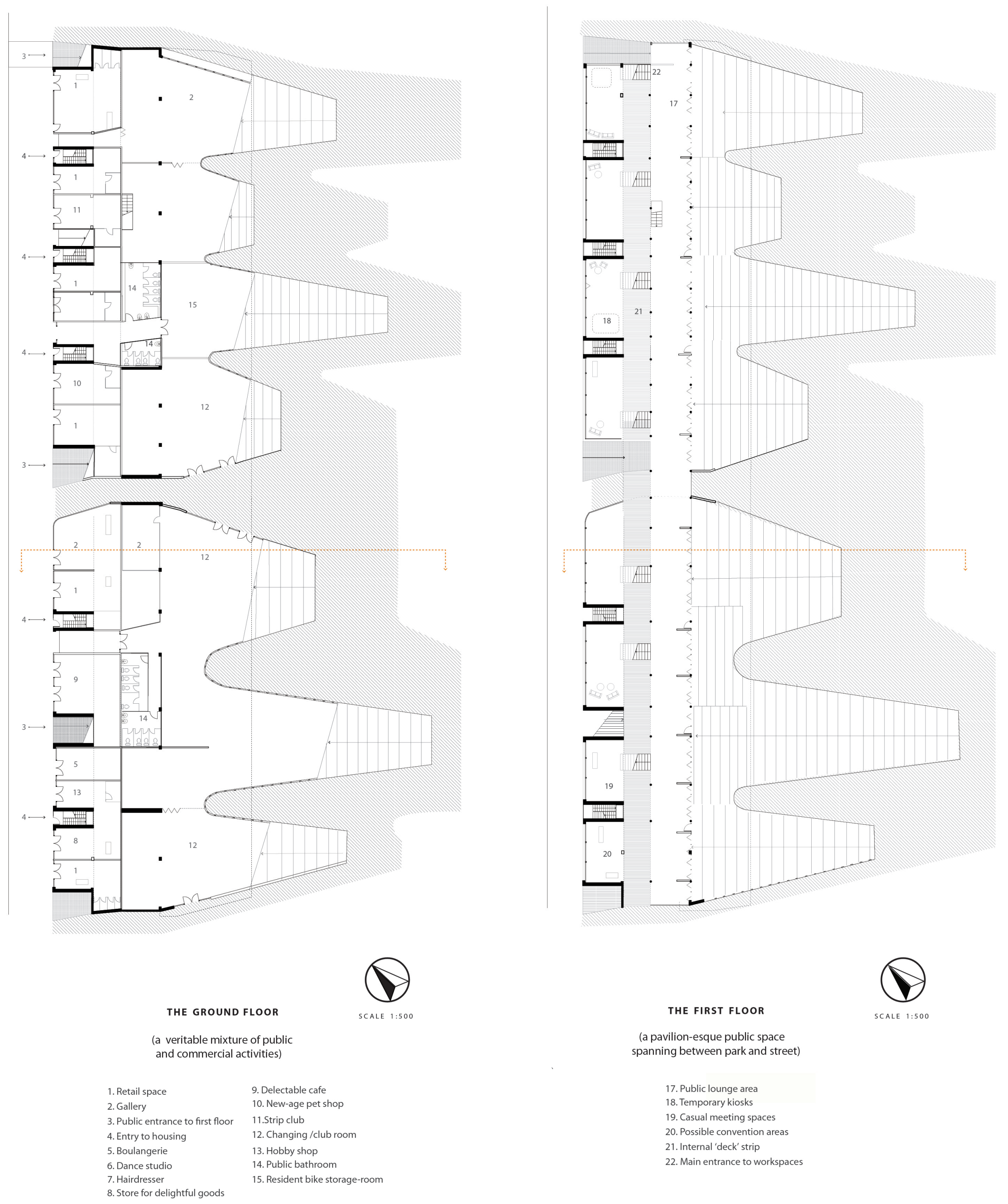
The second, third and fourth floors are comprised of housing and offices/studios, slung over the central chasm. These floors are essentially organised into eight live-work 'clusters', arranged over the three levels and intersected by the vertical ciriculation along the street edge. Each pavilion provides 32 dwellings 16 office/studio spaces.

\section{Housing (right side)}

The second floor consists of studio apartments arranged in groups of two, with a shared entry off the chasm. The third and fourth floors comprise two-bedroom apartments arranged over the two levels, with a shared space in the middle and loft rooms above. Each of the apartments looks out onto the park and meets with central chasm.

\section{Offices/studios (left side)}

Working spaces are spread over the second and third floors - each looking out over the street context on one side, and connecting with the central chasm on the other. On the fourth floor are roof-gardens and outdoor areas for the spaces below.

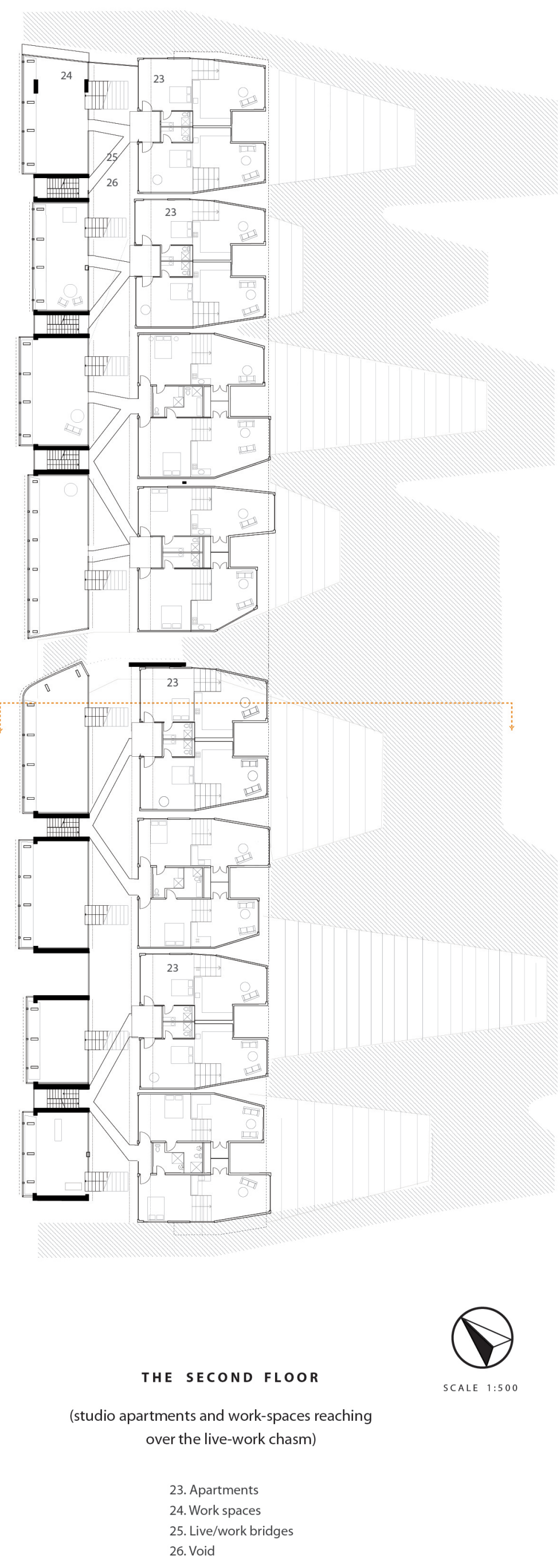



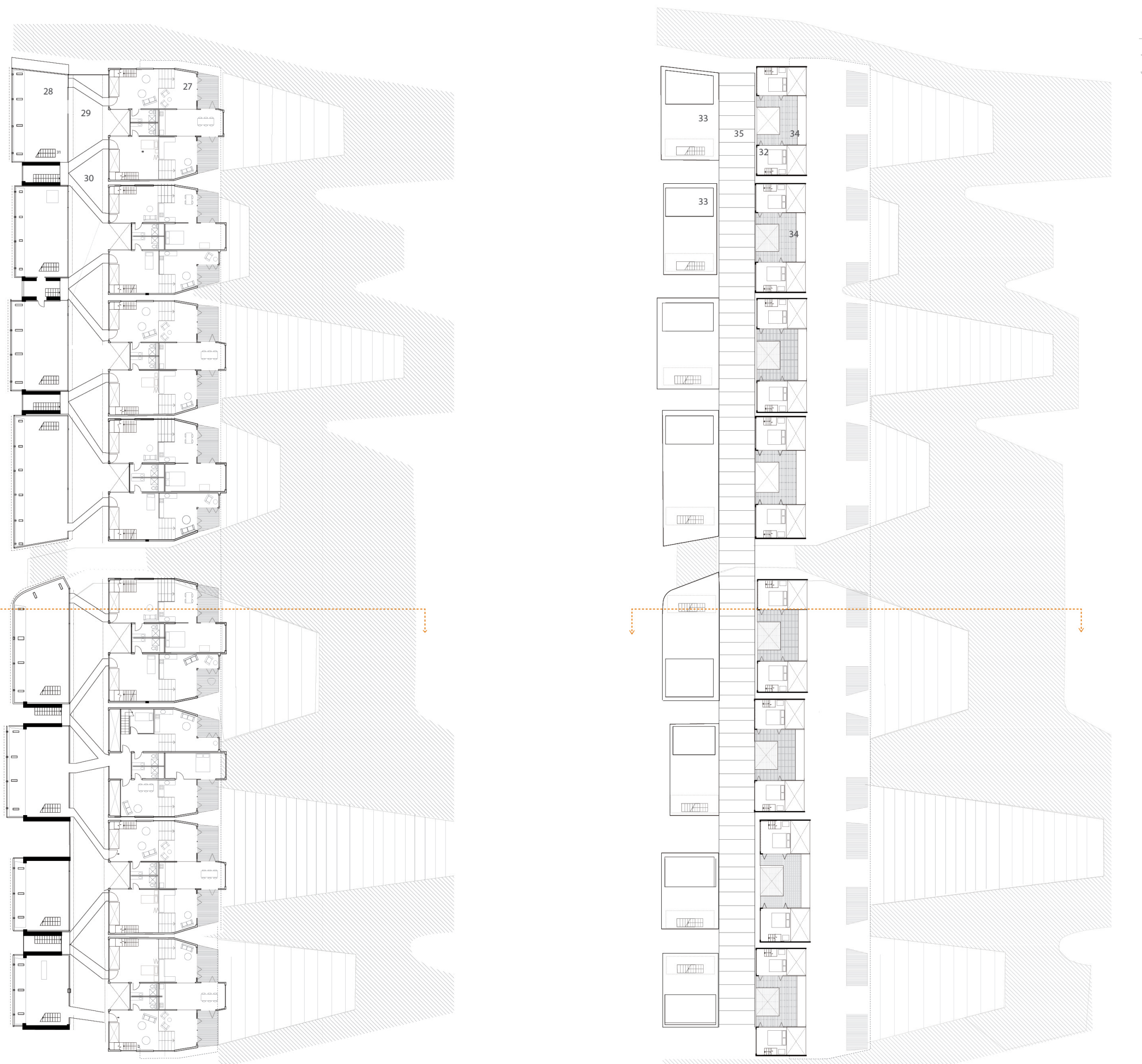

(

THE THIRD FLOOR

(larger apartments with and work-spaces reaching

over the live-work chasm)

27. Apartments

28. Work spaces

30. Void

1. Stairs to roof garden
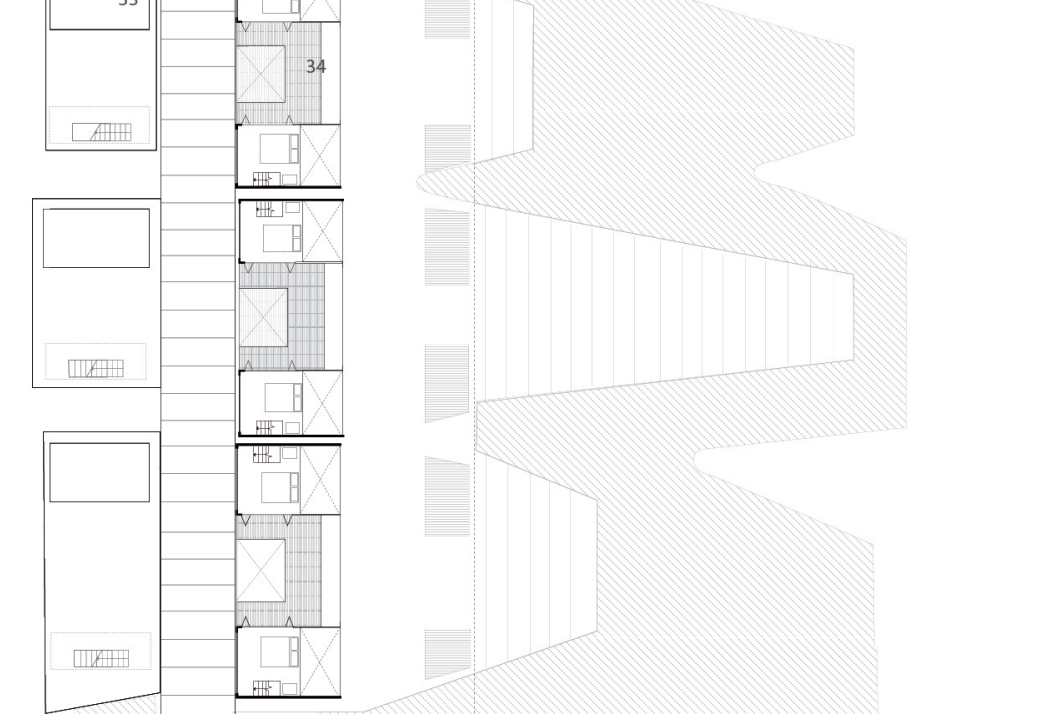

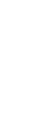

.


Facing the park with a conviction that is equally playful and daring, the architecture lurches out over the open space. The upper mass encroaches on the interlocking terraces and public courtyards beneath, blurring the gap between inside and out, and presenting a dynamic face to the park beyond.

The individually-articulated housing units are poised overhead: breaking down the massiveness and sheer length of the building through their scale, and delivering a certain informality through their scattered configuration. Within, moments of habitation are framed by day; and illuminate the park by night.

Compressed between the terraces and the housing, the band of intermediate space - a public porch-occupies the boundary between inside and out, framing moments of public activity along the length of the building. This elongated strip allows a visual separation between the public space beneath and the private housing above, clearly visible from beyond.

As these different architectural moments collide and are expressly articulated: the housing peering over; the terraces negotiating the ground plane and the central horizontal sliver dividing the masses, a dialogue between private and public, is established spatially, formally and visually.

The building is not an icon, but it is a spectacle: a singular form; a constantly varied strip of habitation and of publics: a housing pavilion, of sorts.

Figure 44 : Park elevation shown from street: Public

pathways are are 'carved' around the building (lower left) allowing it to sit firmly within the site. A continuation from park to street is articulated as the terraces on the park side are echoed by ramps on the street side: effectively wrapping up and through the building. An earlier sketch of the building.

Figure 45 : Sketch of park perpective.
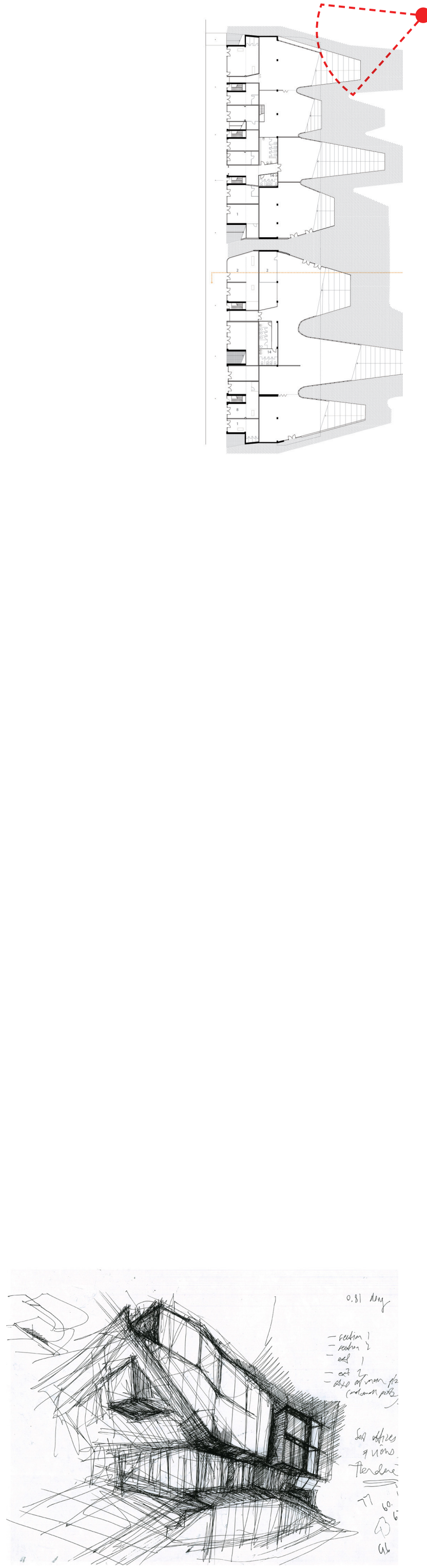


\section{a housing pavillion}

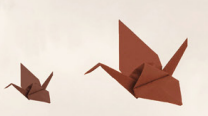

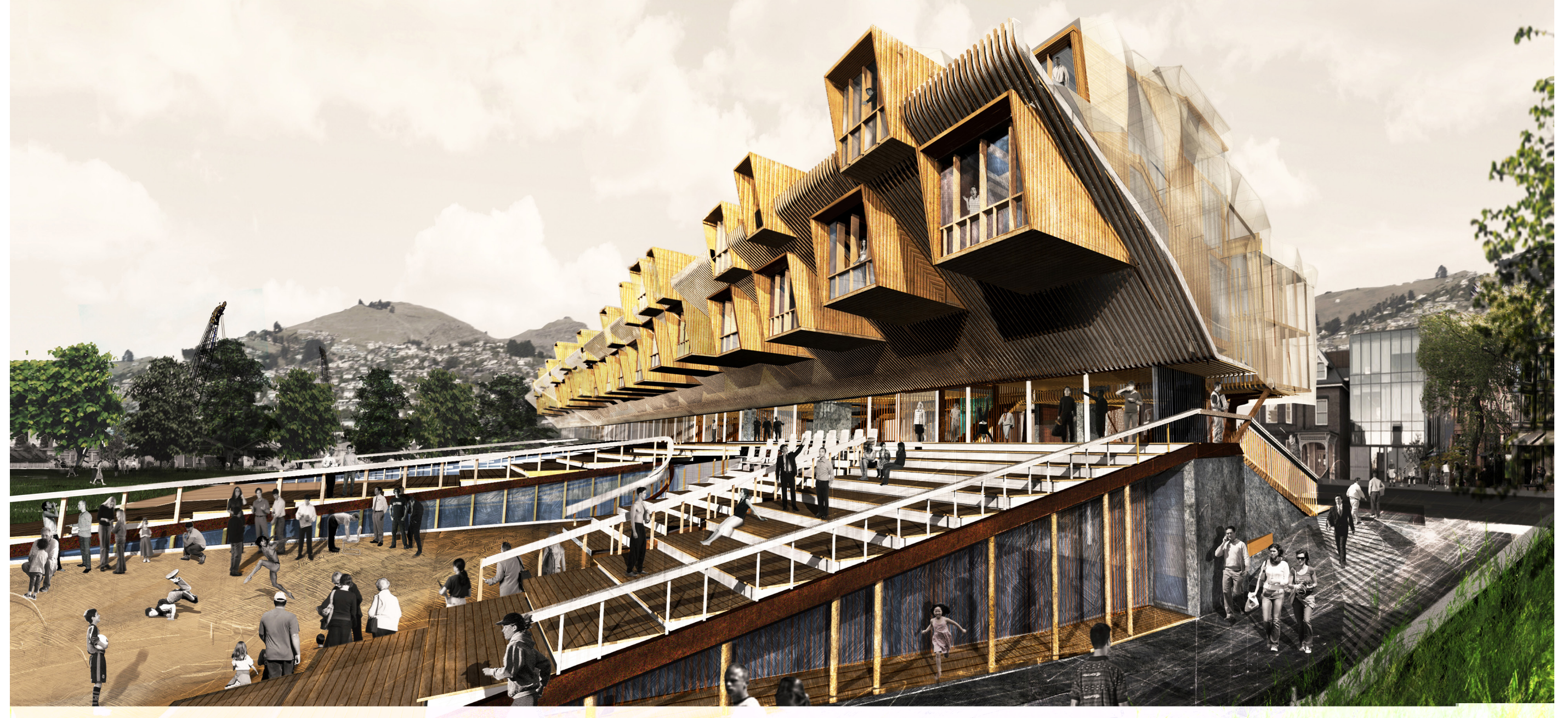


Facing the street, a fabric is created which responds to the grain of the surrounding suburban context, yet does not shy away from a more densified urban language.

The textured street front comprises a variety of commercial spaces, through-ways to spaces beneath the terraces, and ramps which fold down from the first floor, spilling on to the footpath as a spatial and material link to the pavilion gesture on the other side.

Above, the office spaces and their roof gardens protrude: a series of articulated volumes, staggered along the street edge. These are open and light, forming a visual dialogue with the spaces below. Over two floors, white boxes frame moments within these work-spaces, and serve as a compositional gesture to break down the sheer horizontality of the facade, and affording it a certain dynamism. A timber screen wraps over and beneath these offices: a further material link to the park side.

Vertical circulation (up into the housing and work-spaces) visually separates the volumes effectively making up this street edge: the stairwells reading as a series of interstitial, recessed voids along its length.

Like on park side, the public space on the first floor is articulated as a horizontal band: a clear continuity against the more fragmented spaces above and below it.

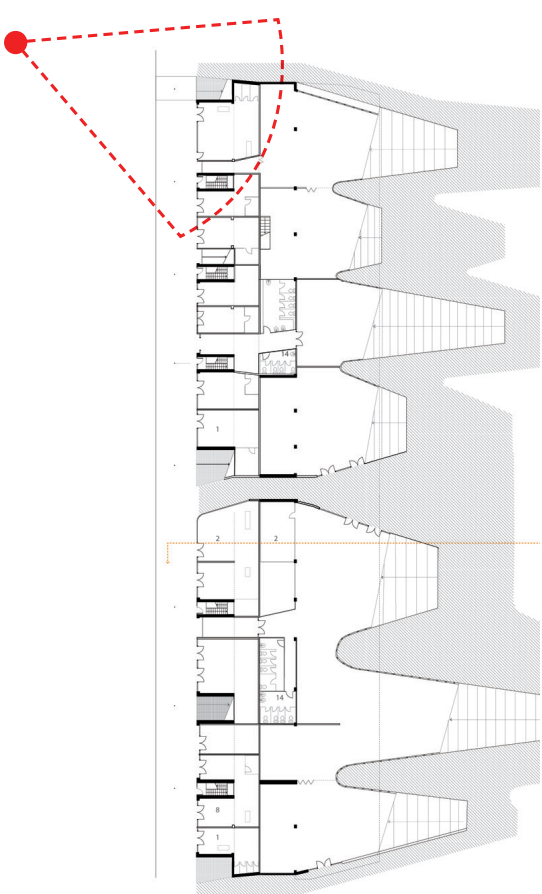

igure 46 : Street elevation: a textured street front with commercial spaces below, offices above, and an articulated public space between them. Ramps fold down from the first floor. 

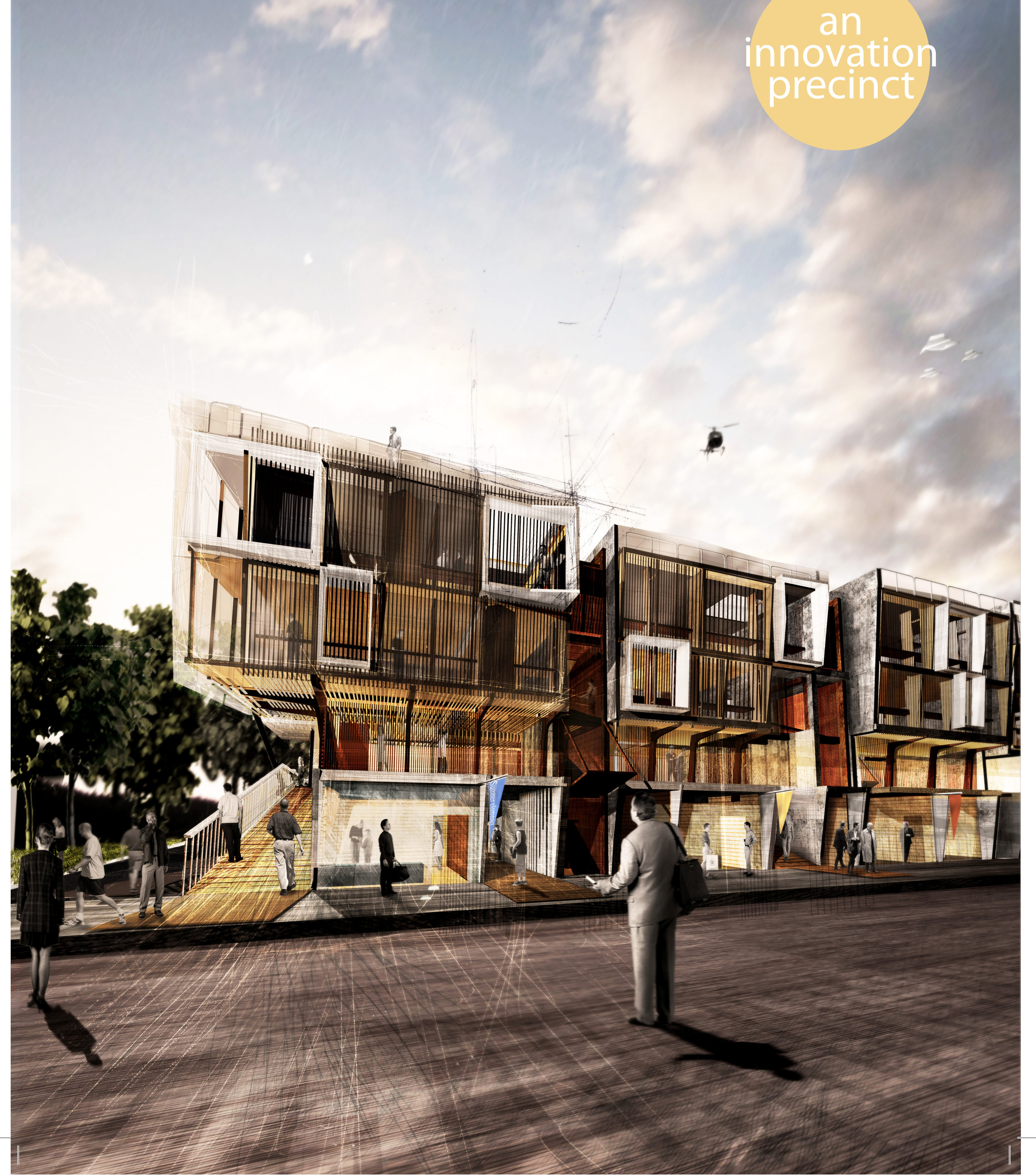

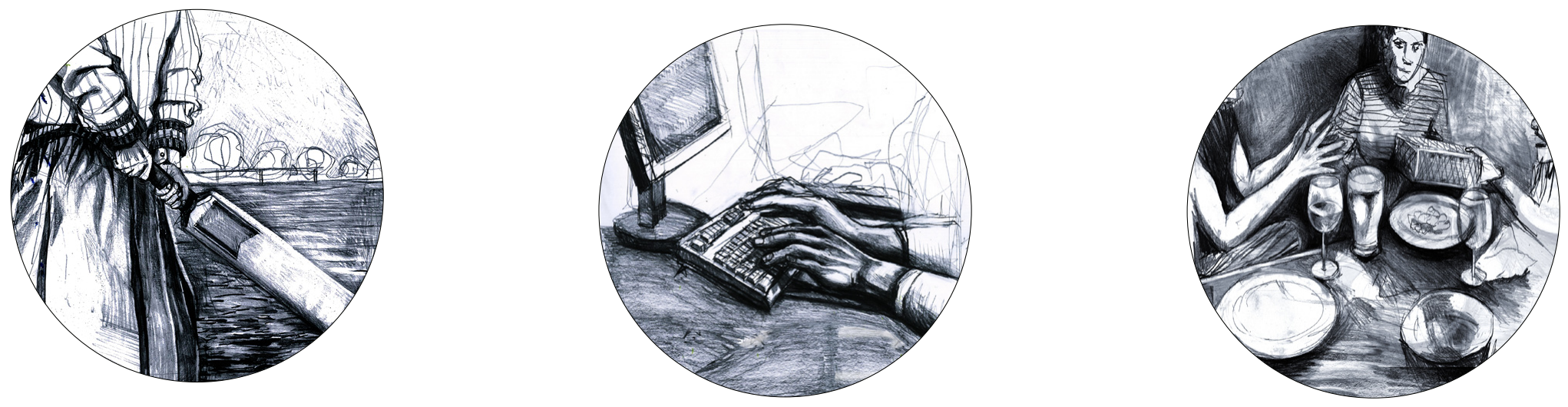

The formal and spatial configuration of the architecture enables a number of functional and social dialogues to develop between 'living' 'working' and 'playing' (public) programmes (see figure 47). These dialogues are played out especially in plan and section, where the rich mixture of activities contained in the building are brought into overlap, intersection and proximity.

\section{Live-work dialogues}

With the advent of working cultures to the suburb, the facilitation of creative, synergic live-work dialogues is essential. These programmes are brought into fundamental dialogue as they are slung across the central chasm. At each of the upper levels, this arrangement engenders a series of publicly-intersected 'live-work' units. Collectively, these units form eight live-work'clusters' along the length of the building, each comprising four apartments and several shared workspaces (see figures 48-49).

Notably, with the intermediate void between the living and working parts of the building, flexibility is enabled. This means the housing and office spaces may be occupied by the same tenants, or remain more separately utilised. Either way, a dialogue is fundamentally enabled between the two.

\section{Public-private dialogues}

Public and private spaces are interfaced at multiple points in the building, particularly through the central chasm and the permeating connection between park and street.

By way of the central chasm,for example, the public space on the first floor becomes engaged with the live-work dialogues above: contributing further to the possibility for creative encounters (see figures 50-51, overleaf).

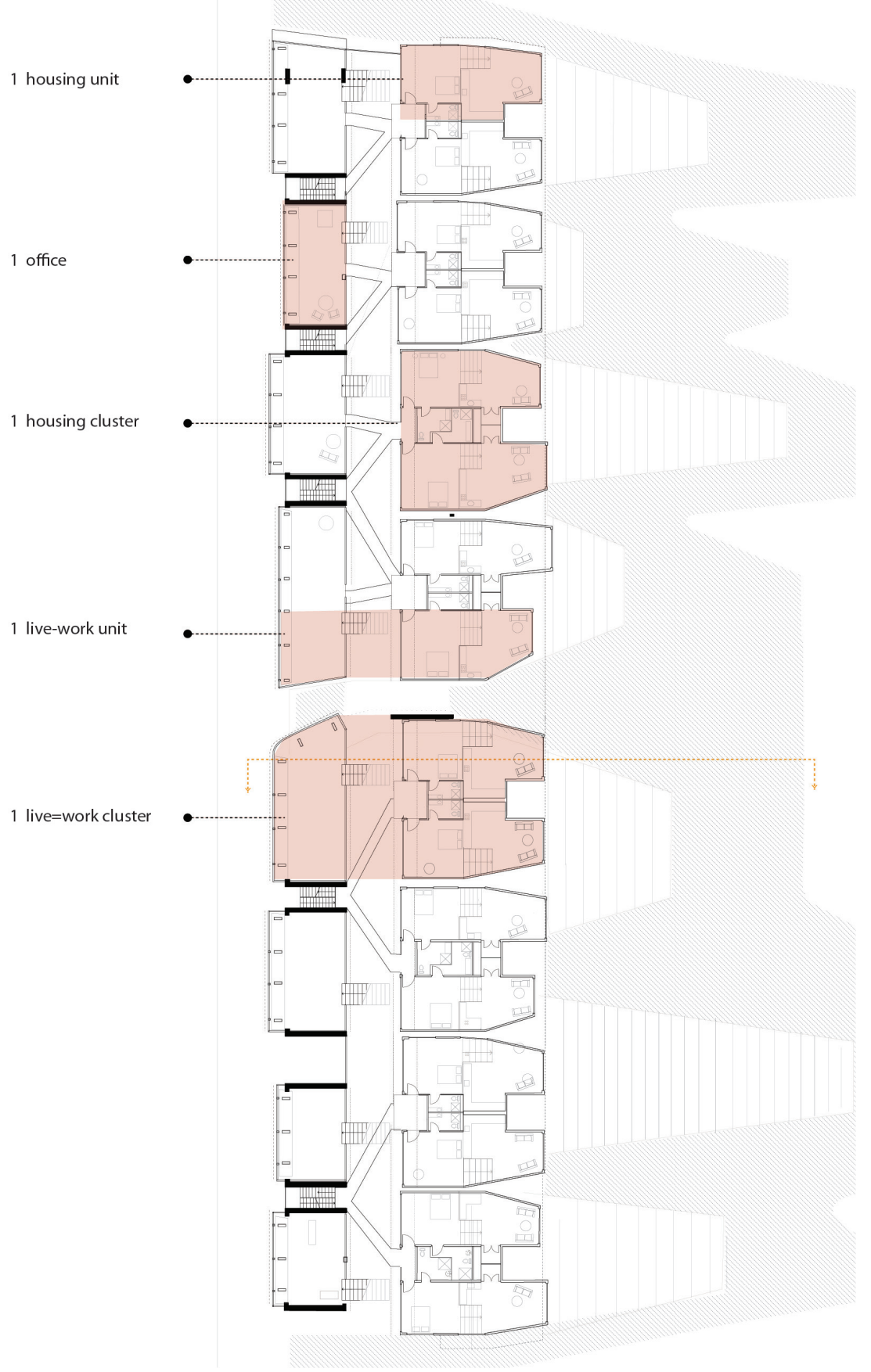

Figure 47 : Top: Live-work-play drawings. The configuration of 'live,' 'work' and 'play' programmes facilitates a number of social interactions and creative dialogues. In this way, the architecture becoming a sub-centre microcosm, letting this rich mixture of programme overlap and intersect.

Figure 48 : Above : Plan showing live-work dialogues 

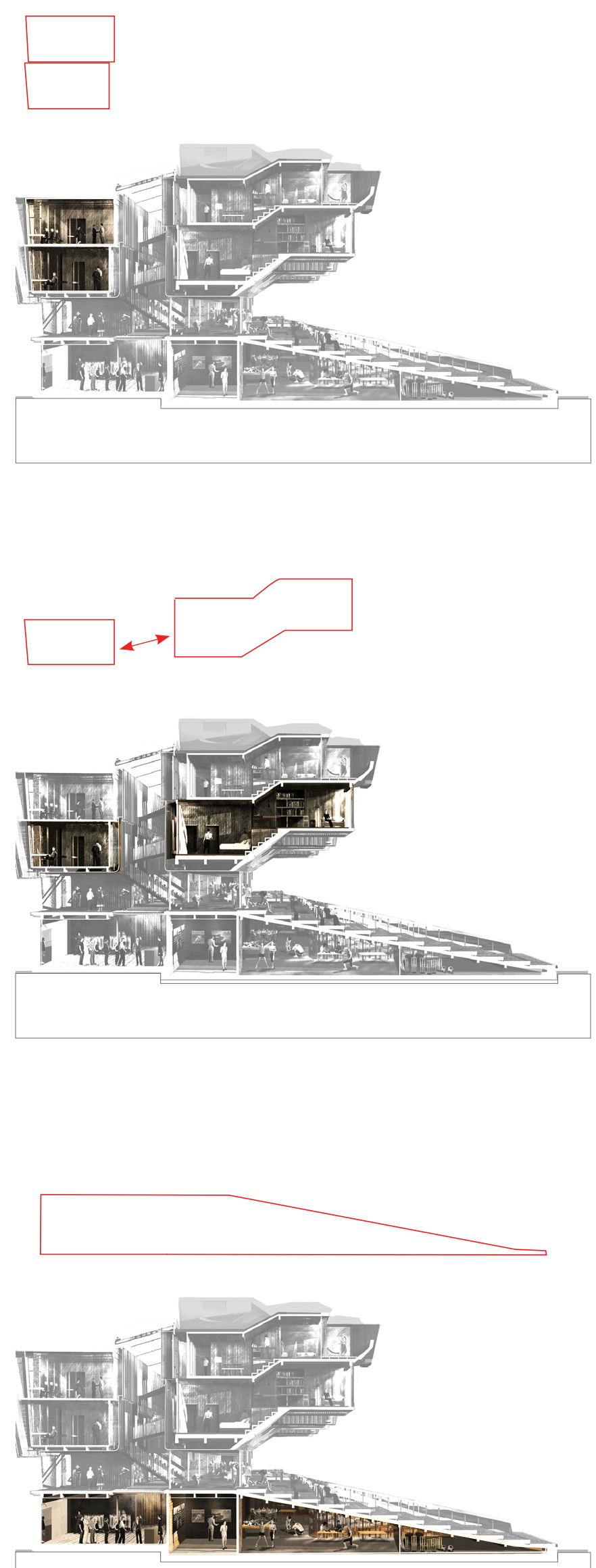
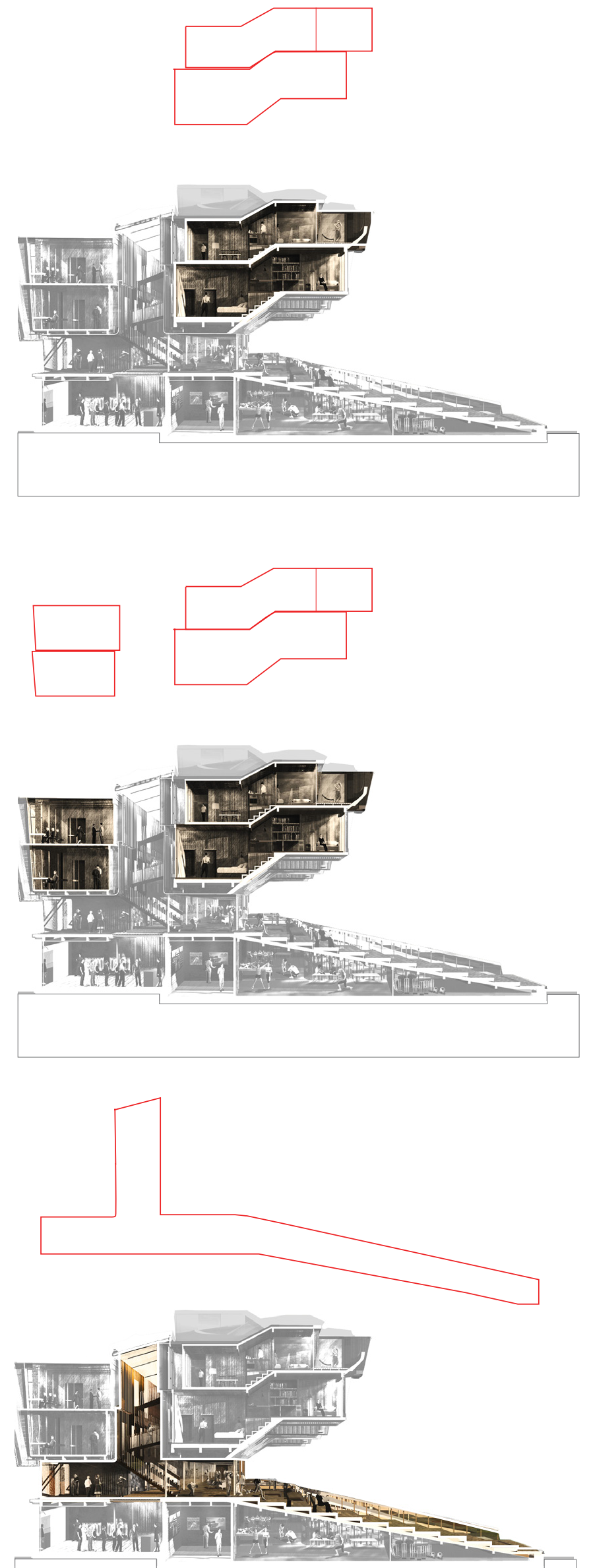

Figure 49 : Six sectional diagrams. Top row: offices, iving
spaces. Second row: a live-work unit; a live-work cluster; Bottom row: commericlal, leisure and semi-public spaces; central public'chasm 


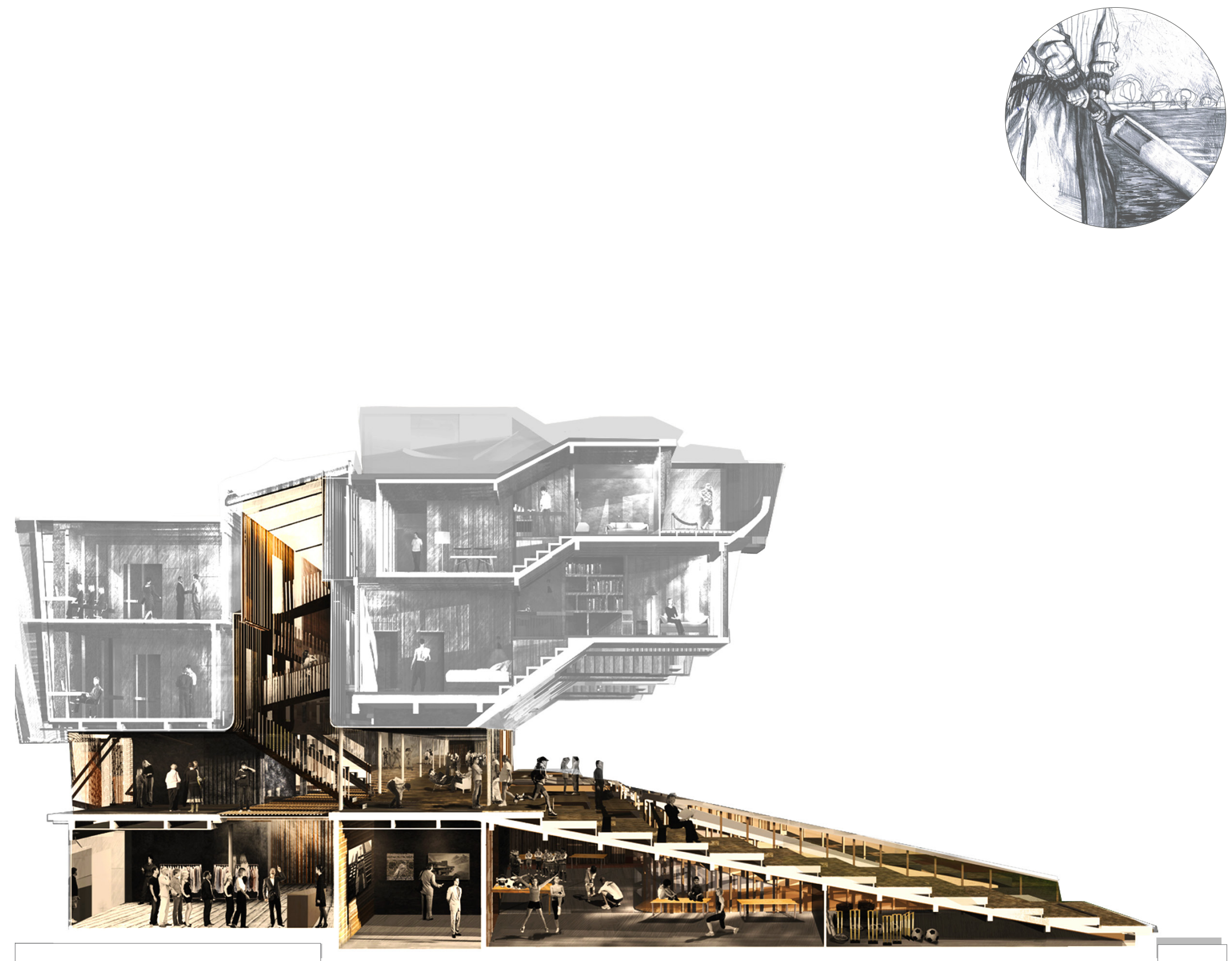

\section{Public}

Figure 51 : Public (and semi-public) spaces consist of the

commerical and recreatational spaces on the ground floor, and the central chasm which links with the first floor 'porch' and terraces. 

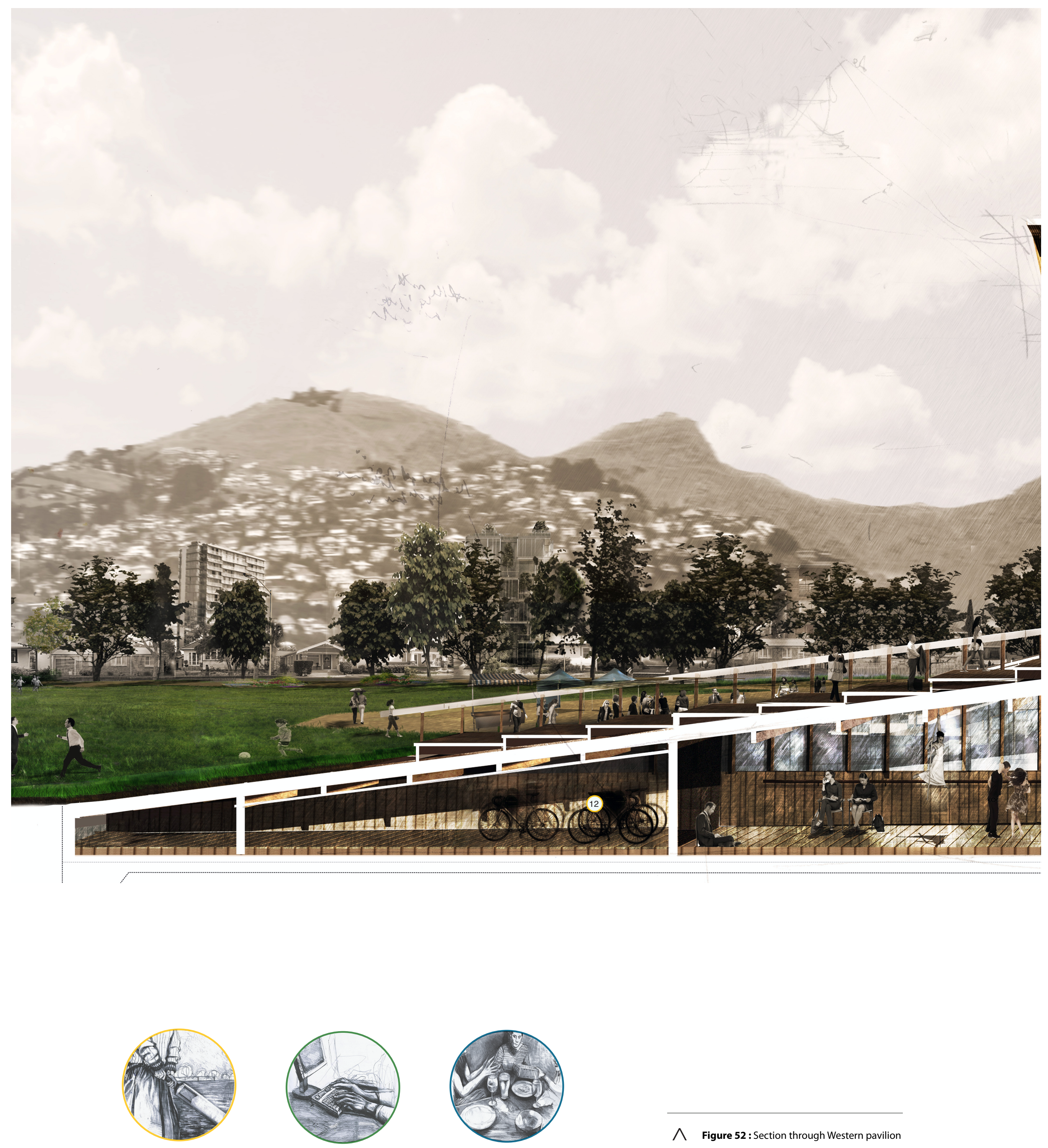

$\bigwedge$ Figure 52 : Section through Western pavilion 

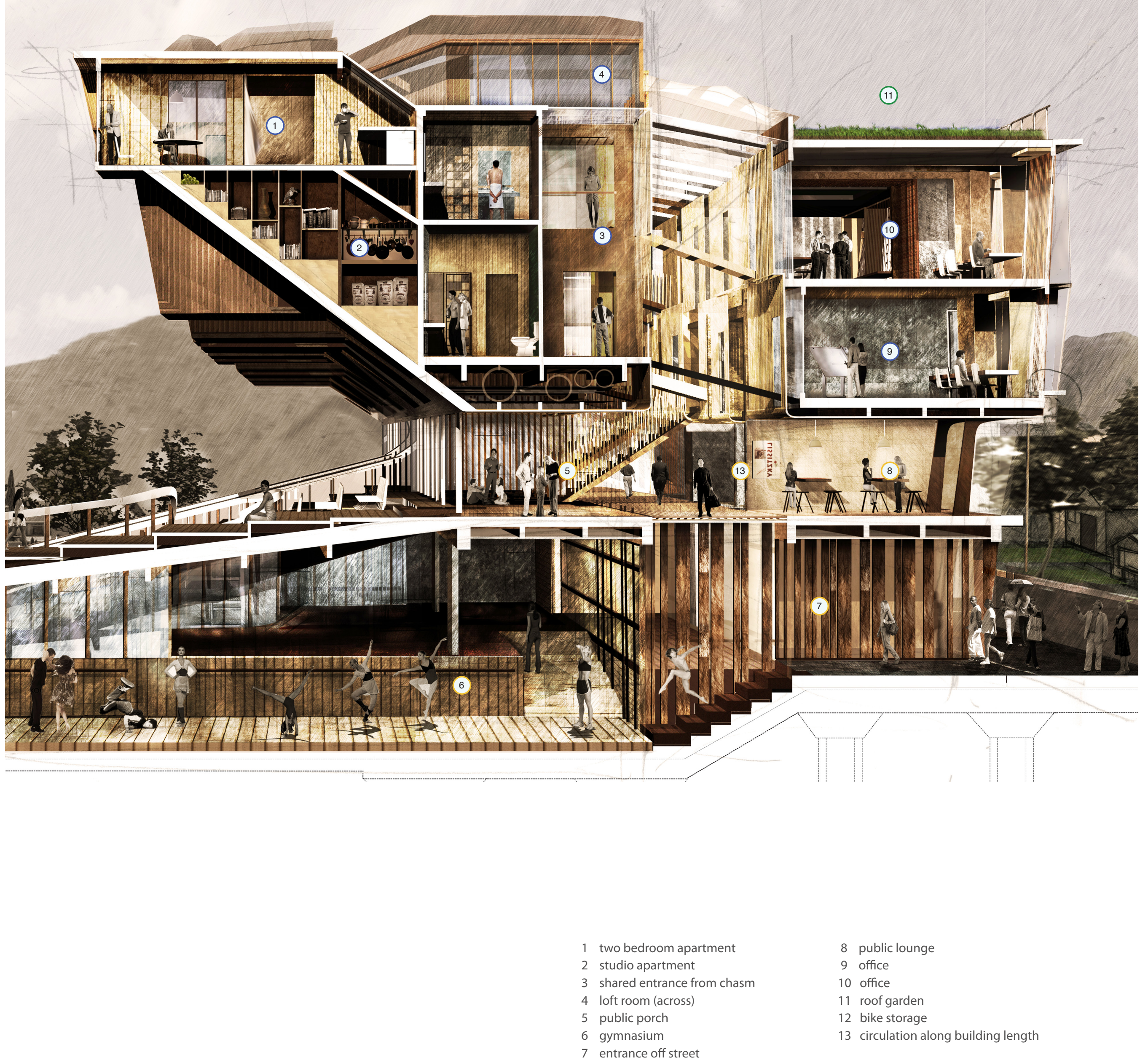


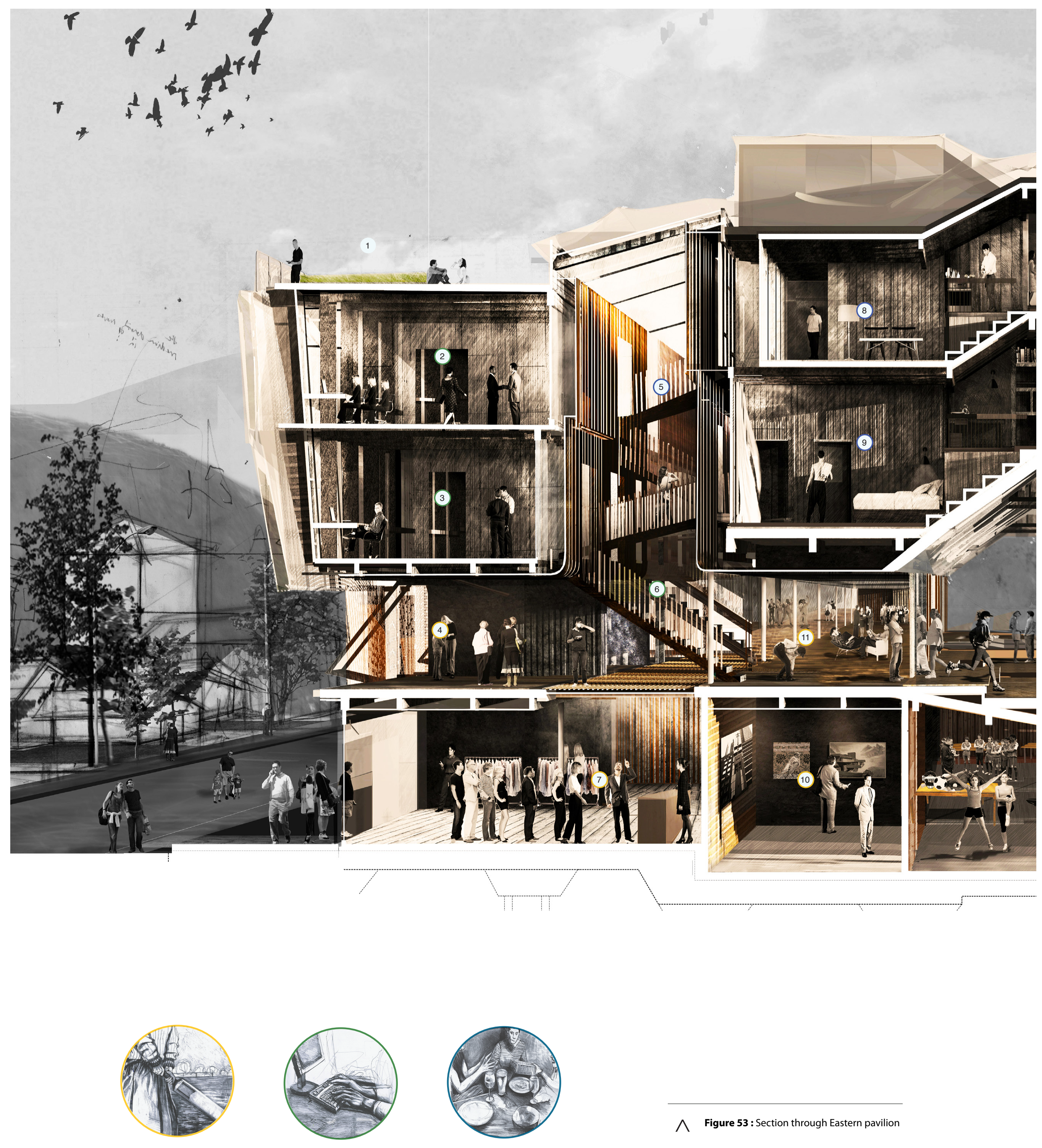



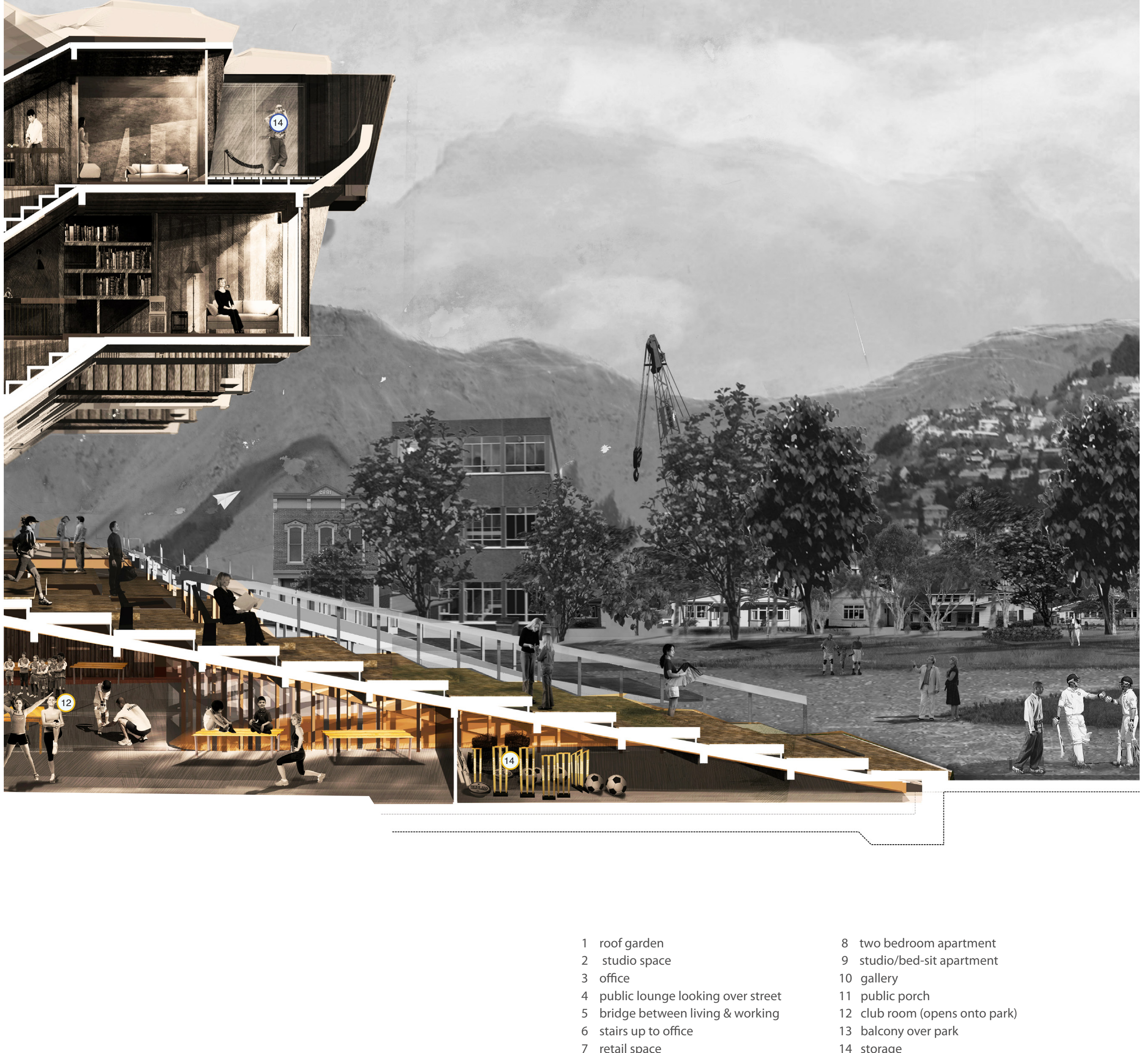

8 two bedroom apartment

9 studio/bed-sit apartment

10 gallery

11 public porch

12 club room (opens onto park)

13 balcony over park

14 storage 


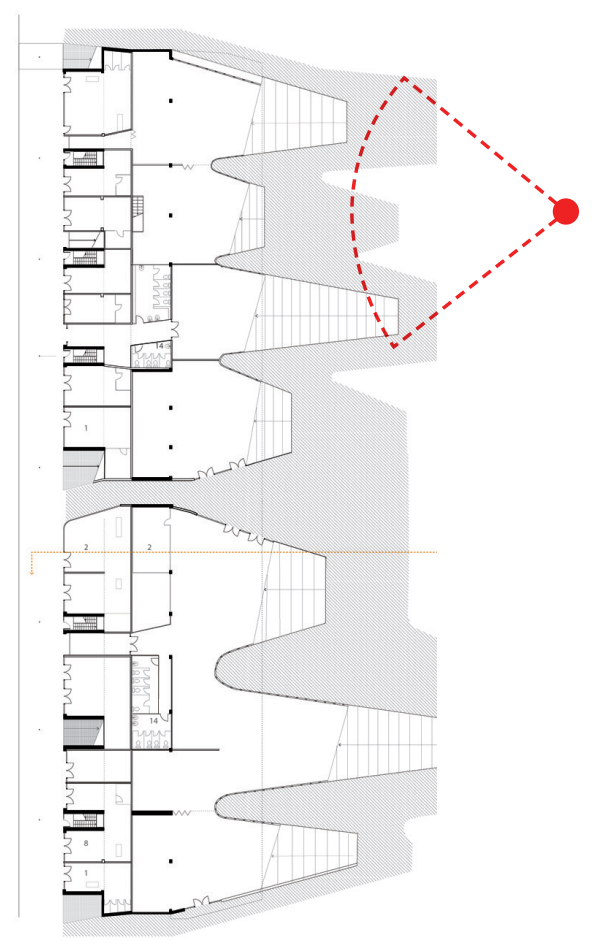

At night, the architecture becomes a suburban light, The housing units, puncturing through, are illuminated as a dynamic visual strip of dense habitation. The horizontal band through the middle of the building reads as a clear void between the masses it separates, framing silhouettes of social activity.

As the terraces draw the open space of the park up into the building, activity is illuminated beneath them.

Cutting into the ground plane as they do, the terraces allow the spaces between them to feel more intimately-scaled - despite the vastness of the architecture itself. 


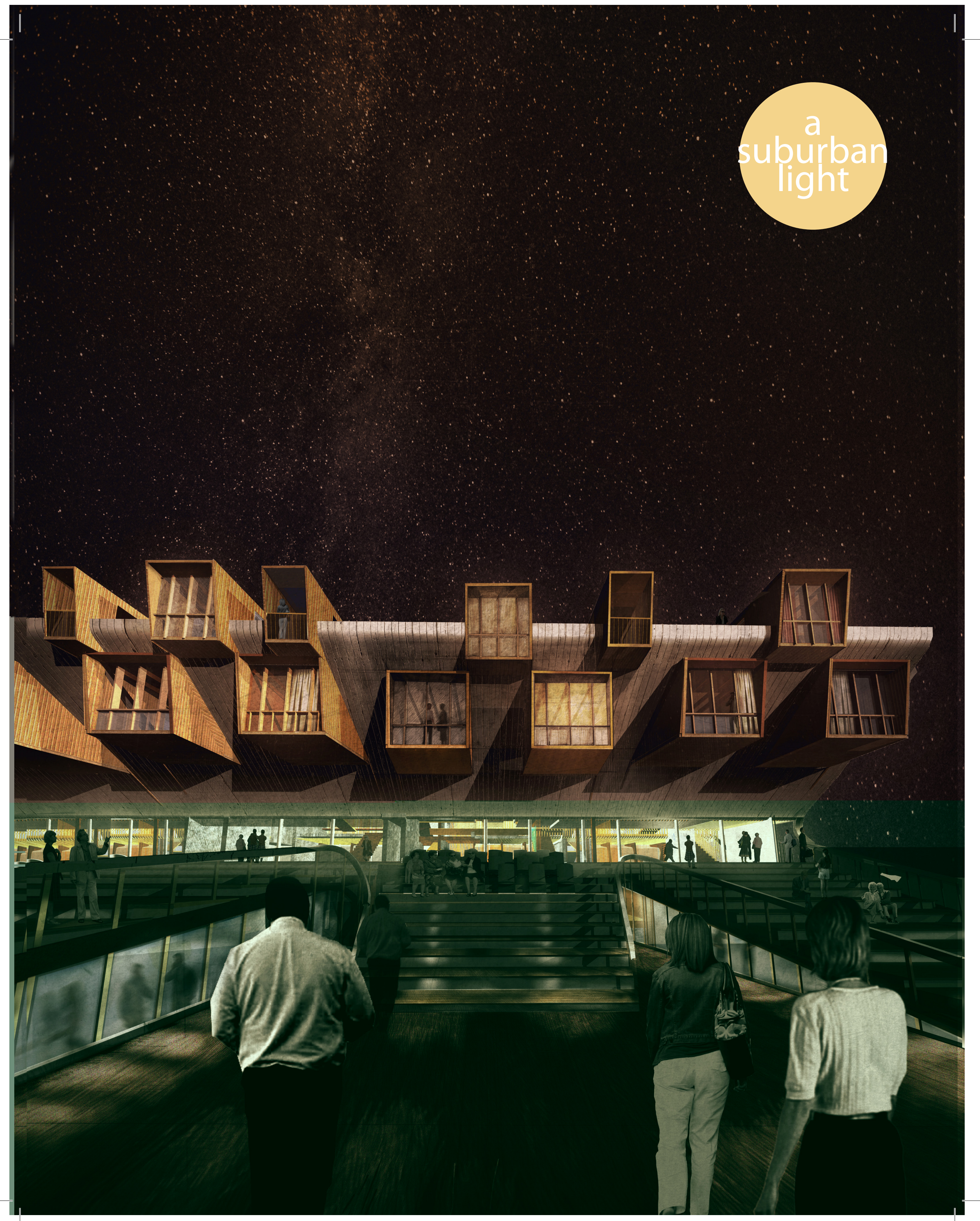



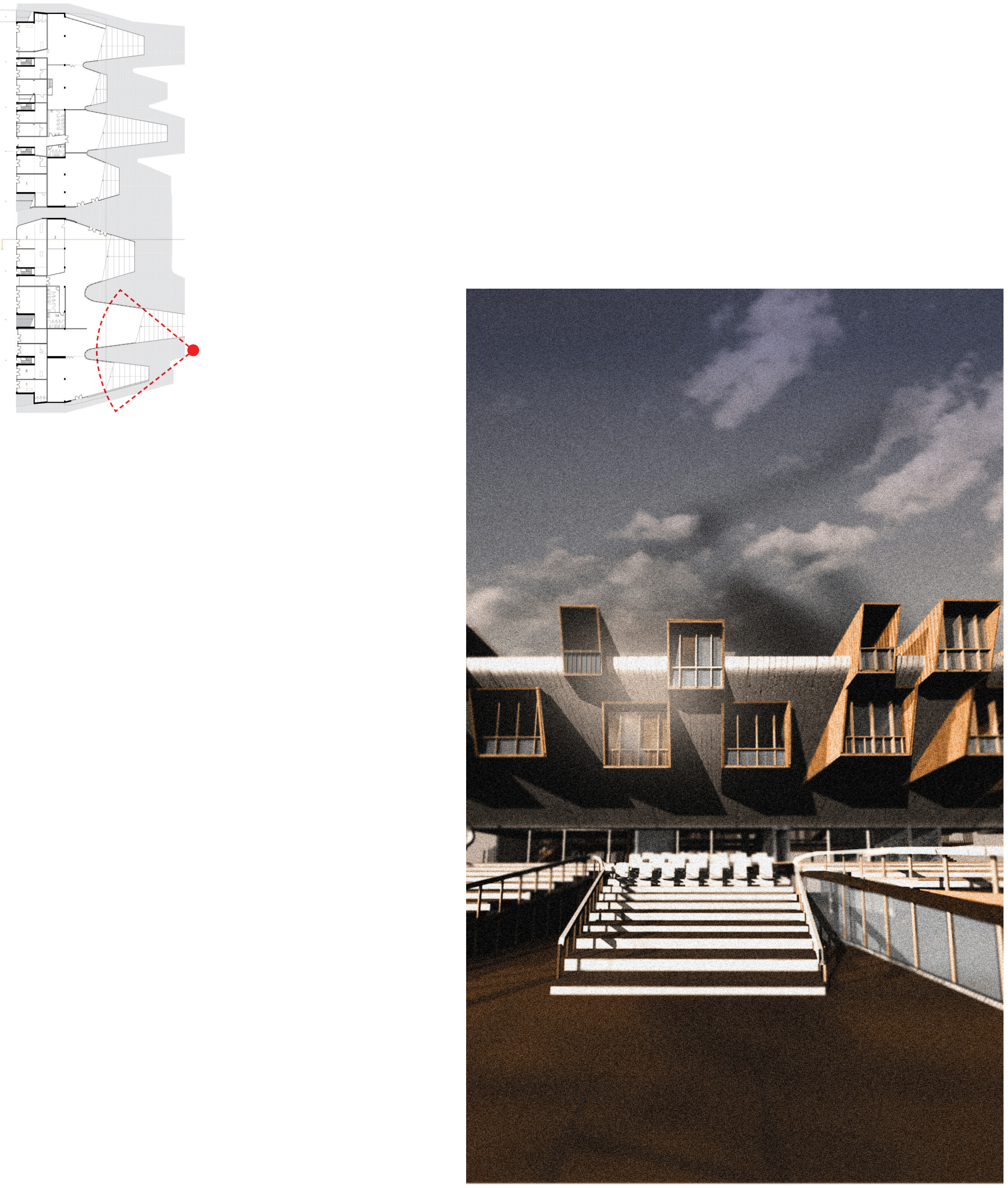

$>$ Figure 55 : Exterior view from the public forecourt 

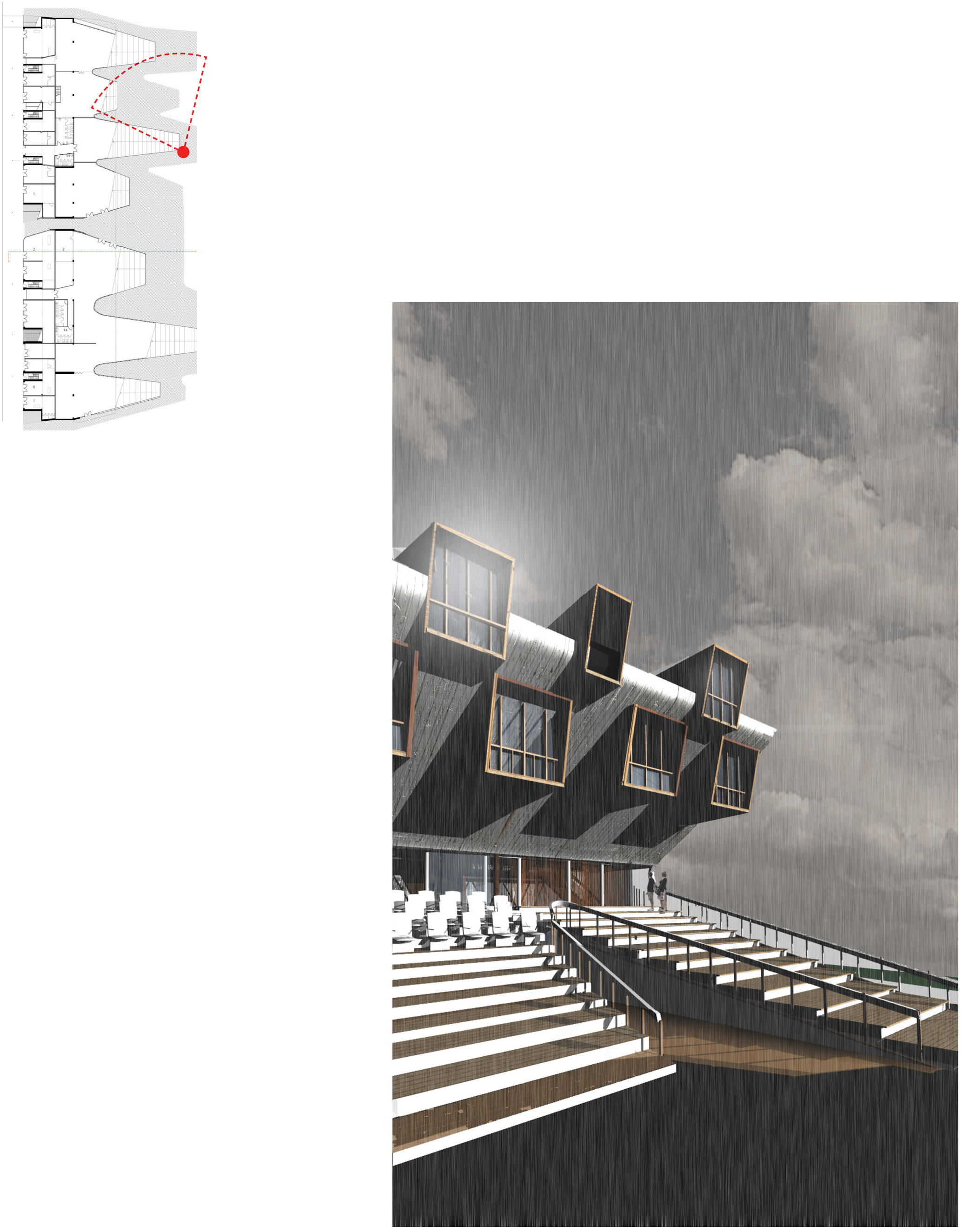

$>$ Figure 56: Exterior perspective 


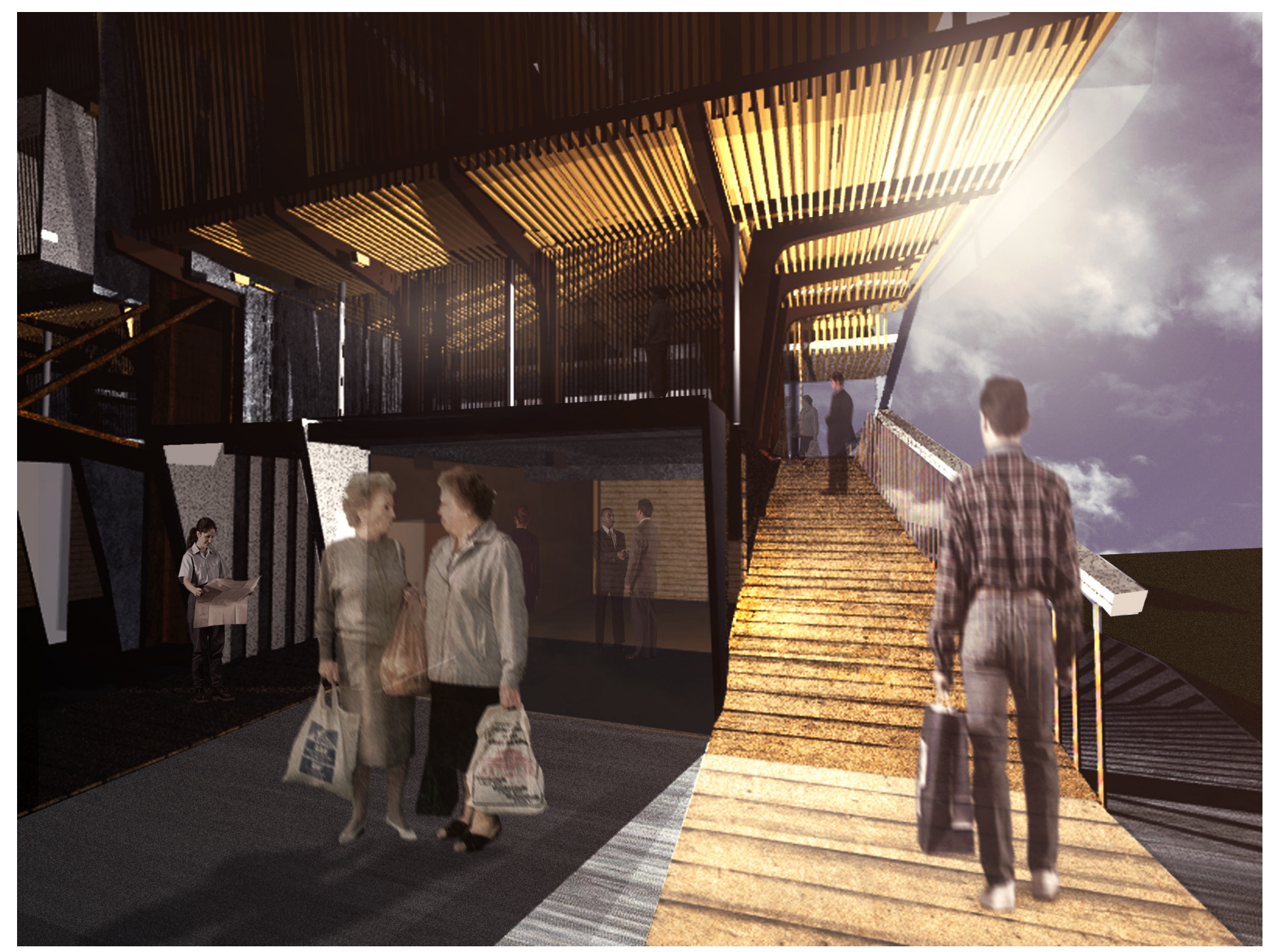

$\bigwedge$ Figure 57 : Access way to first floor from street. The material palette allows the building to operate somewhere between street edge and pavilion.

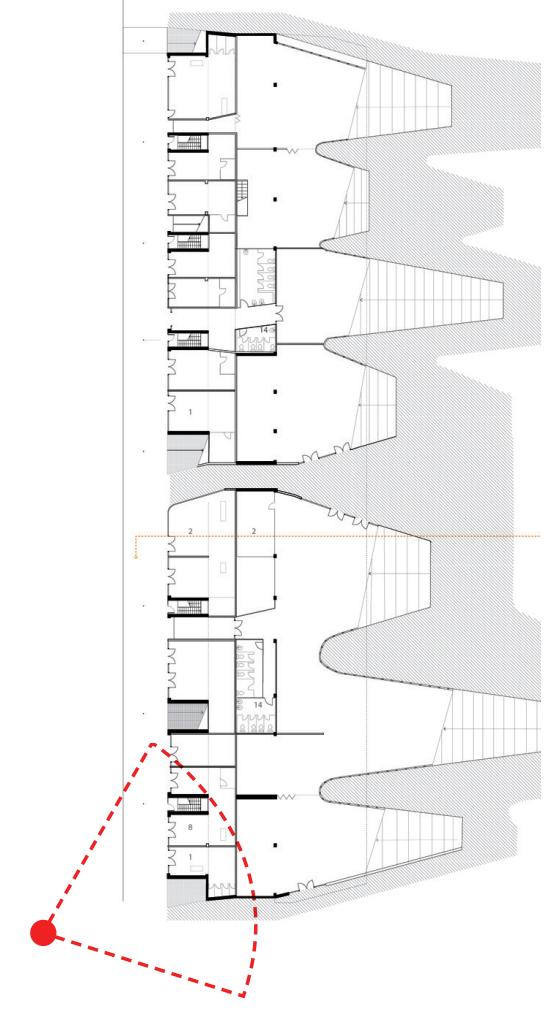



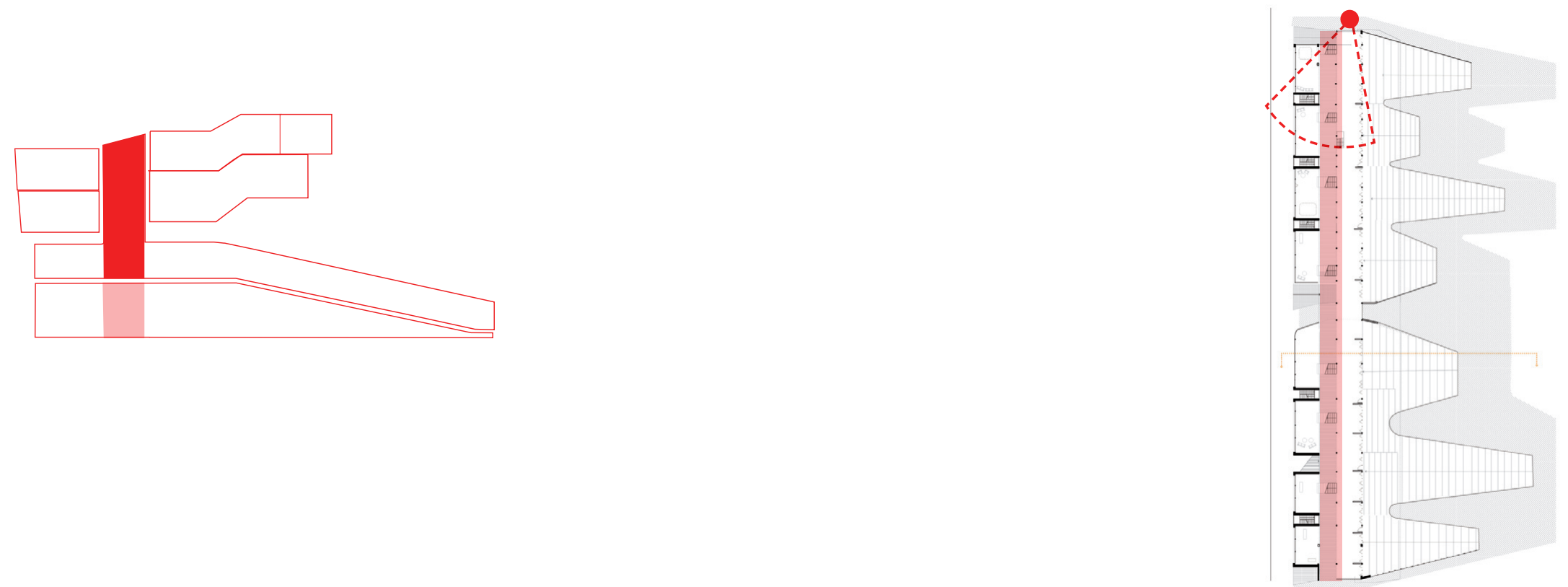

Internally, the live-work chasm becomes a vital social space, linking with the terraces on one side, looking over the street on the other. Compartmentalised by the stairwells into smaller public 'lounges', this unprogrammed space caters for a rich mixture of activities.

Light floods into the chasm from above and from the sides, enabling an open, semi-outdoor feel. This is reinforced by the materiality: timber screens that wrap around the upper volumes and form the ceiling the first floor, and an internal decking 'strip' which runs the length of the building - relating to the terraces.

The chasm is also illuminated above by the work spaces (right) and housing (left). The activity within these spaces, visible behind the timber screens, adds to the essentially social atmosphere of this central space. 


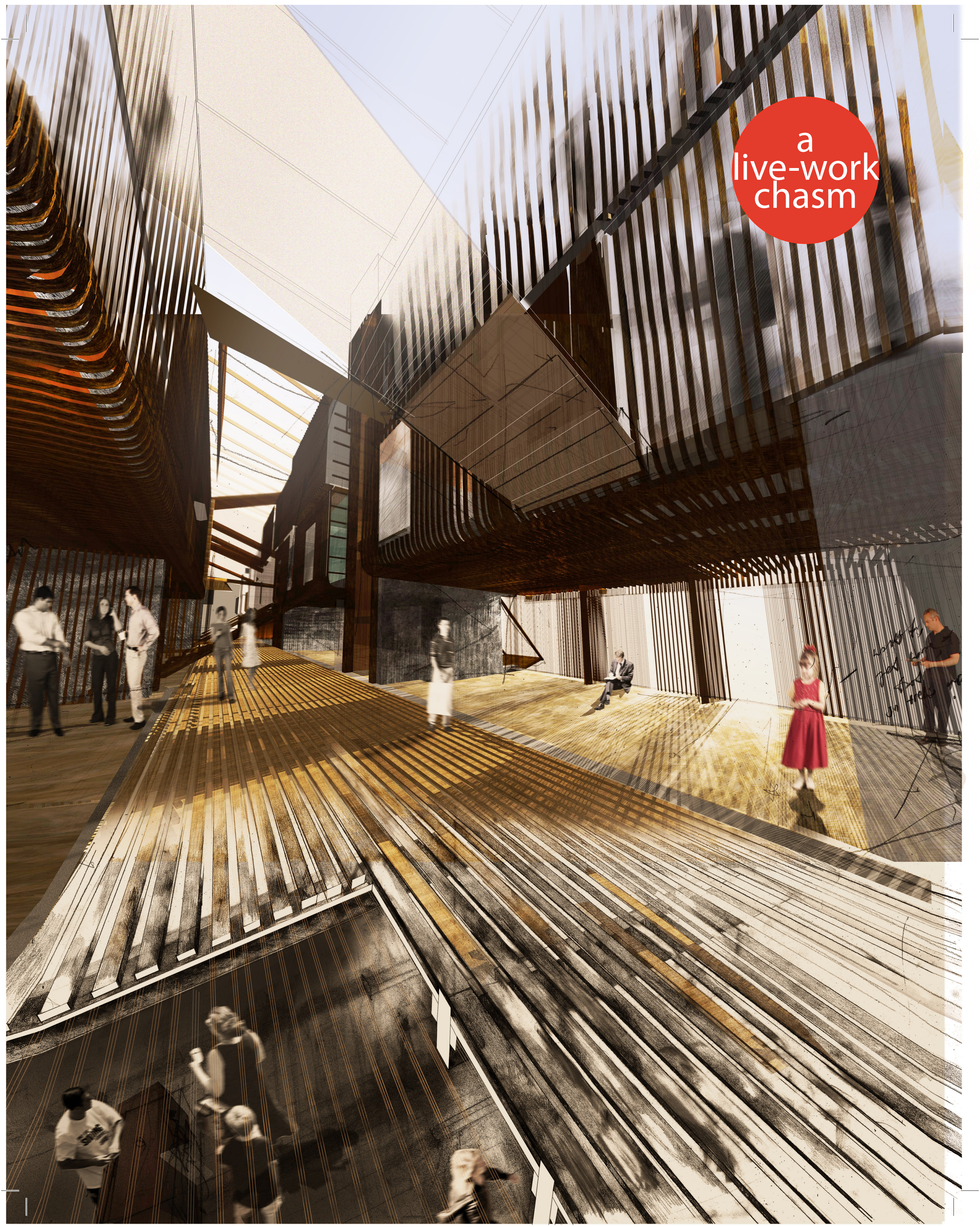




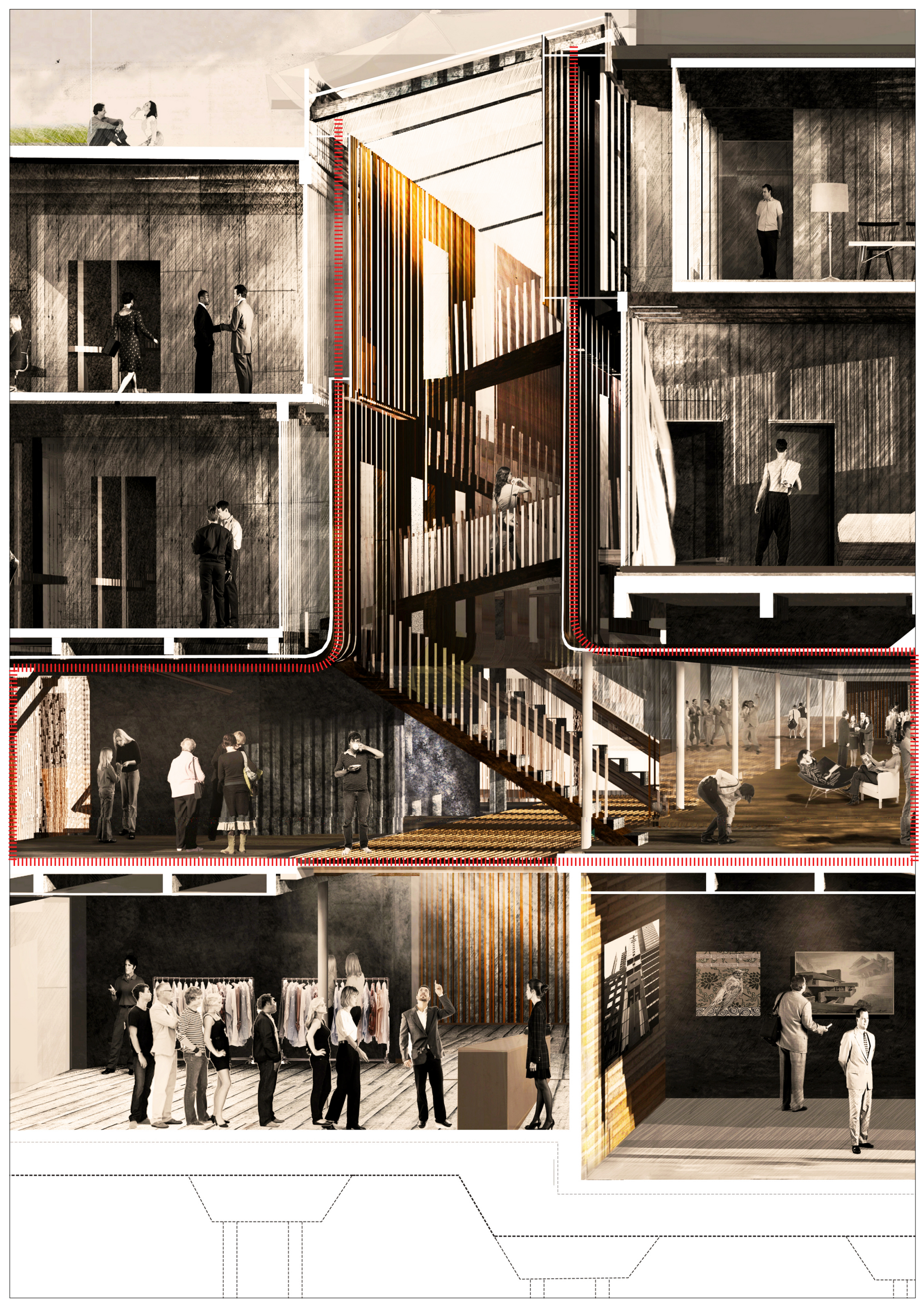

A Figure 59: Sections through the live-work chasms in the Eastern (left) and Western pavilion. Timber screens wrap around the upper volumes, permitting privacy for the living and working spaces. These screens are punctured by bridges and access ways: creating a visual display of live-work dialogues from beneath. 


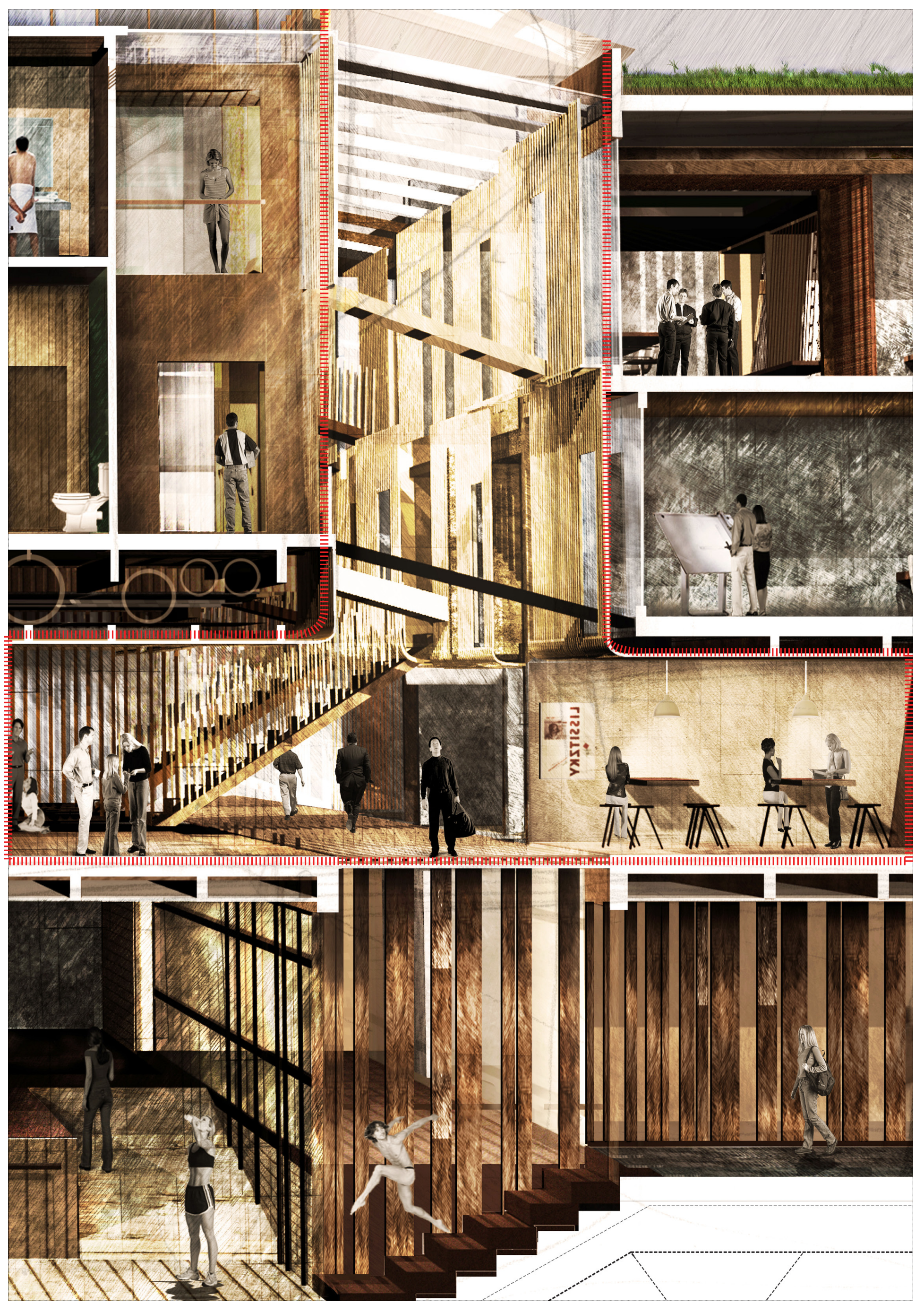



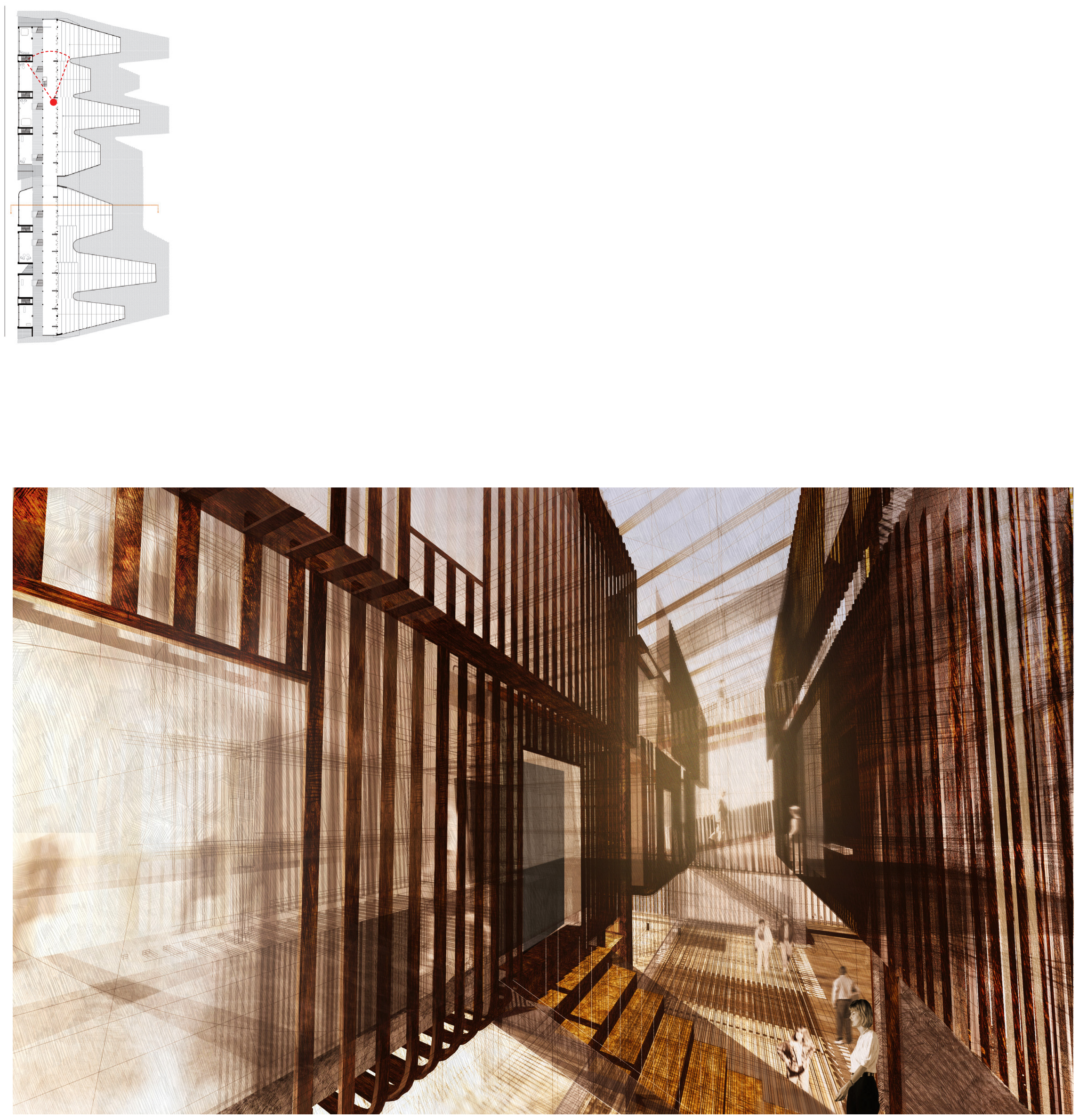

Figure 60 : Interior perspective looking down the live-work chasm. Activity in the offices is obscured by the timber screens (left). A continuity is enabled between living and working spaces through materiality. Different volumes are articulated as they front onto the chasm. 

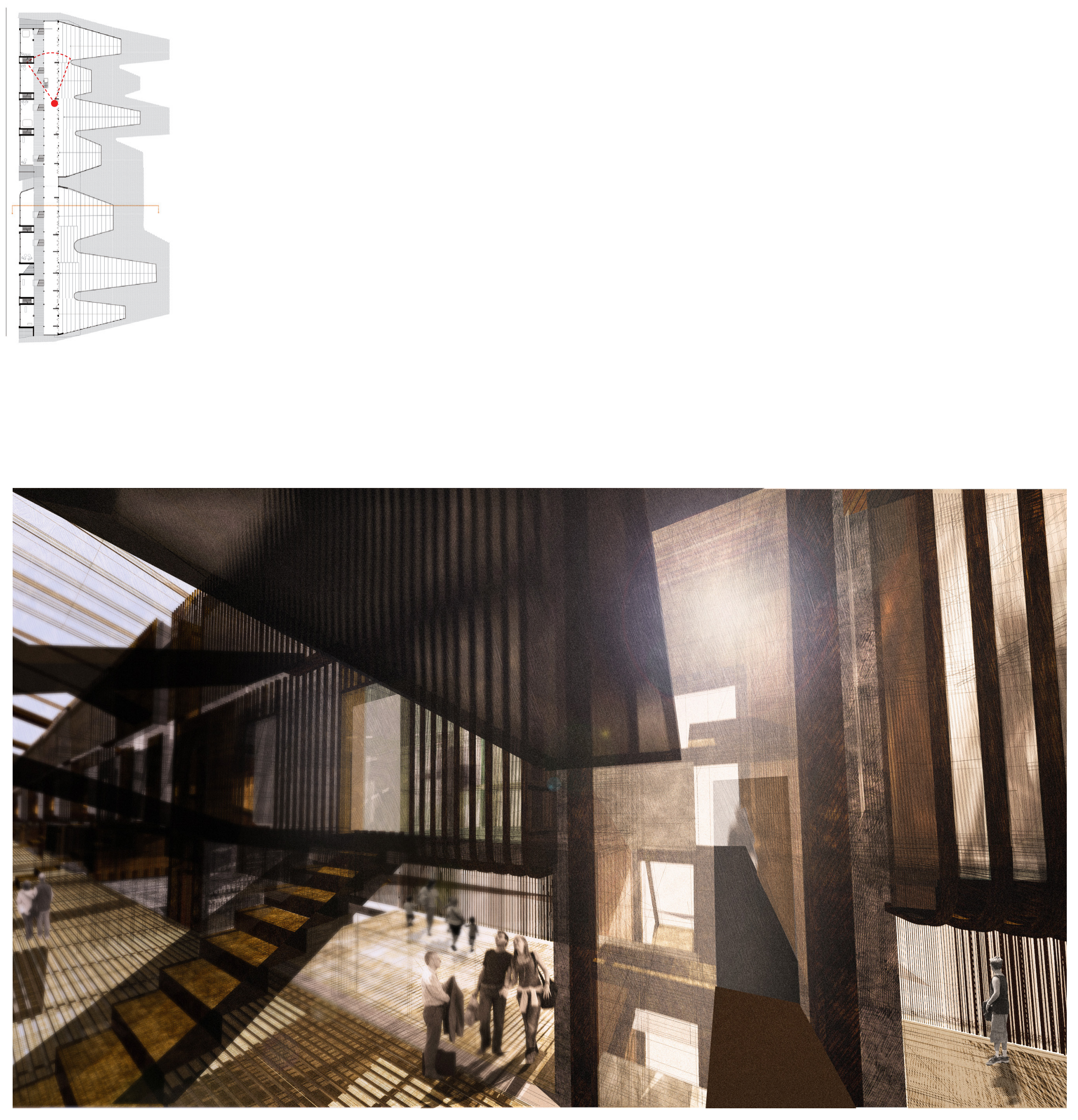

$\triangle$ Figure 61 : Interior perspective looking across live-work chasm, through stairwell and out to the street. Below, public lounges cater for a variety of activities, and the internal decking strip runs the length of the building. 


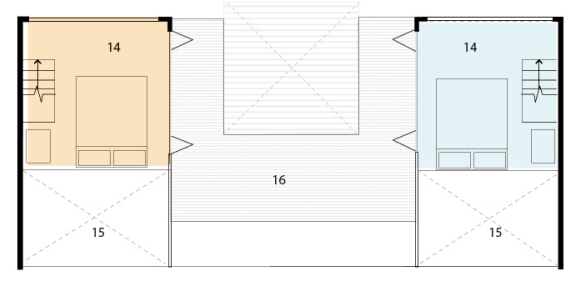

UPPER

14. bedroom

15.void
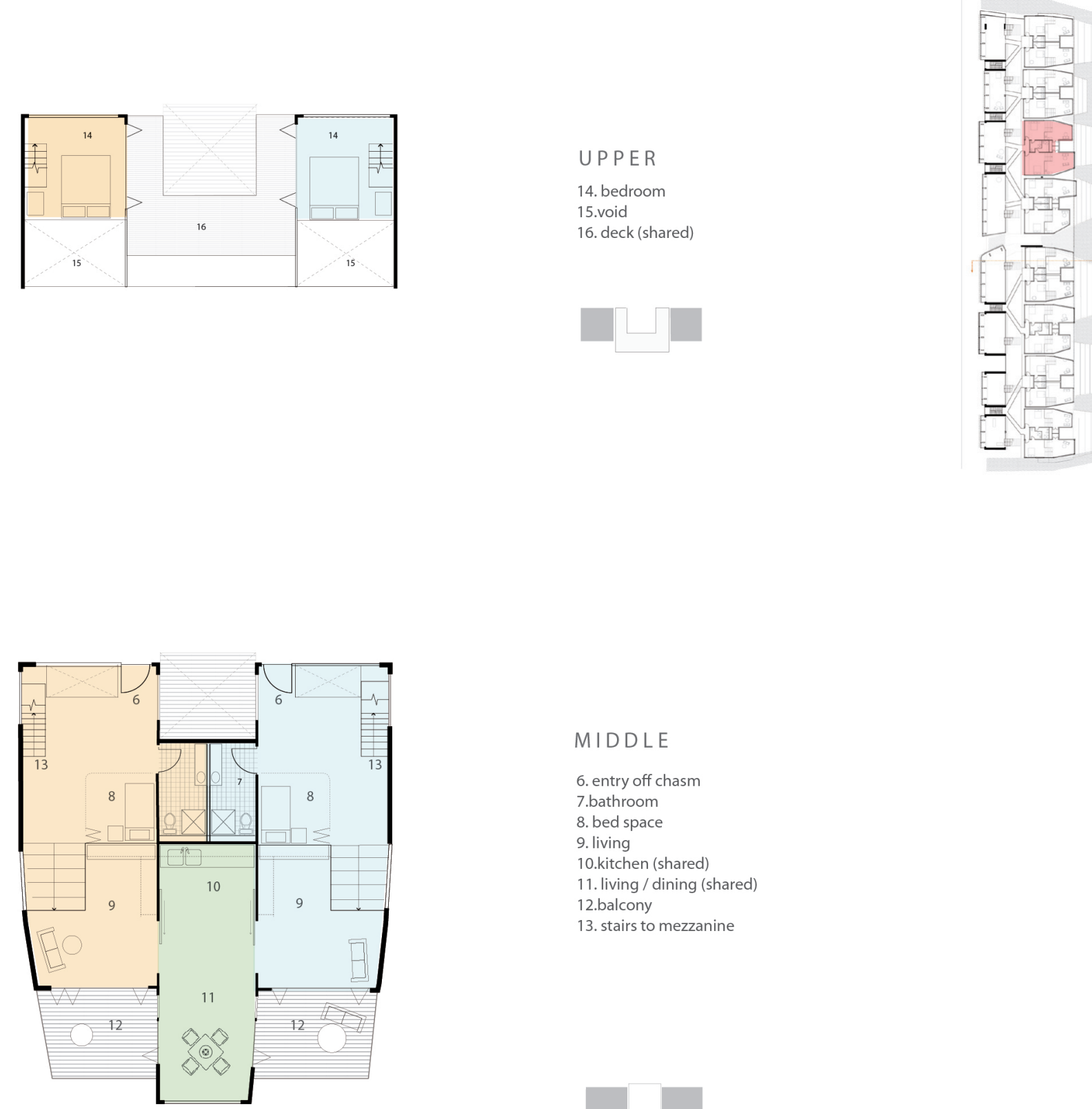

MIDDLE

6. entry off chasm

7.bathroom

8. bed space

10.kitchen (shared)

11. living / dining (shared)

12.balcony

13. stairs to mezzanine

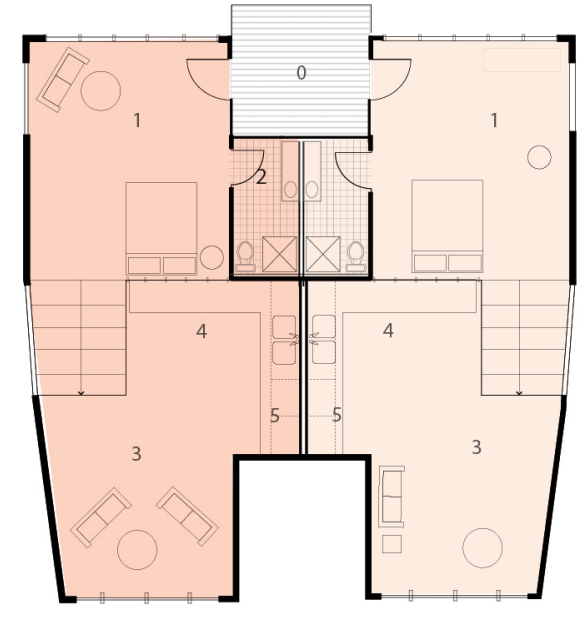

LOWER

0 . entry off chasm

1.bedroom

2.bathroo

3.living

5. storag

6.services shaft

Private

Shared

Scale - 1:200 
M

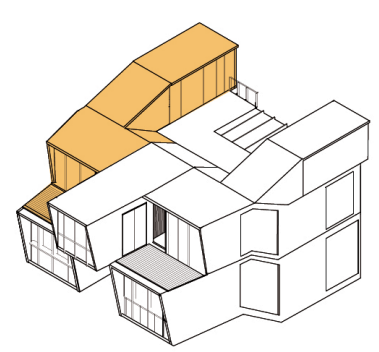

tive $(1)$

M
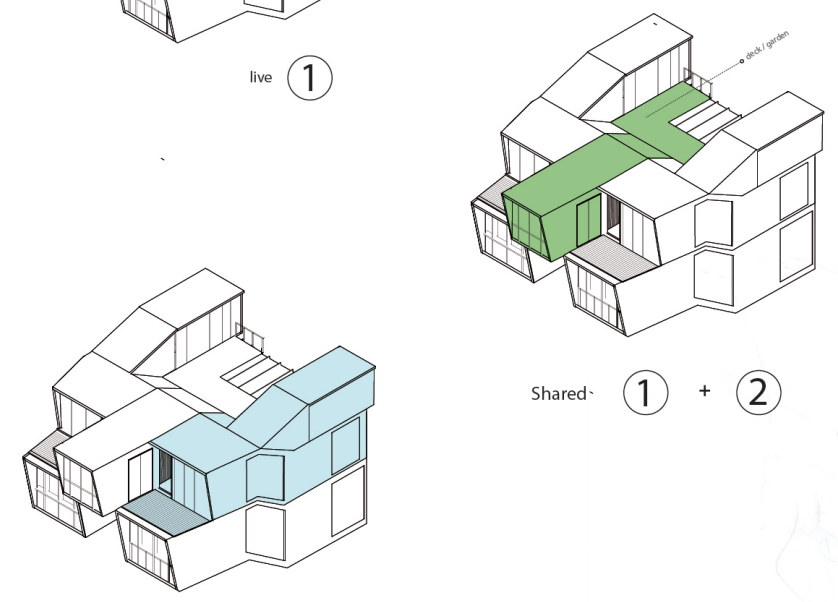

shared. $(1)+2$

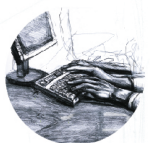

we (2)

快

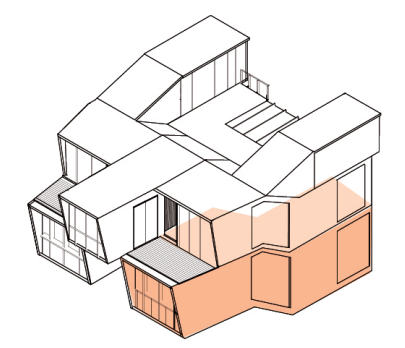

the (3)

in

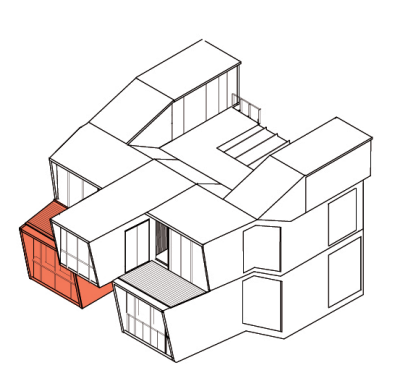

the (4)

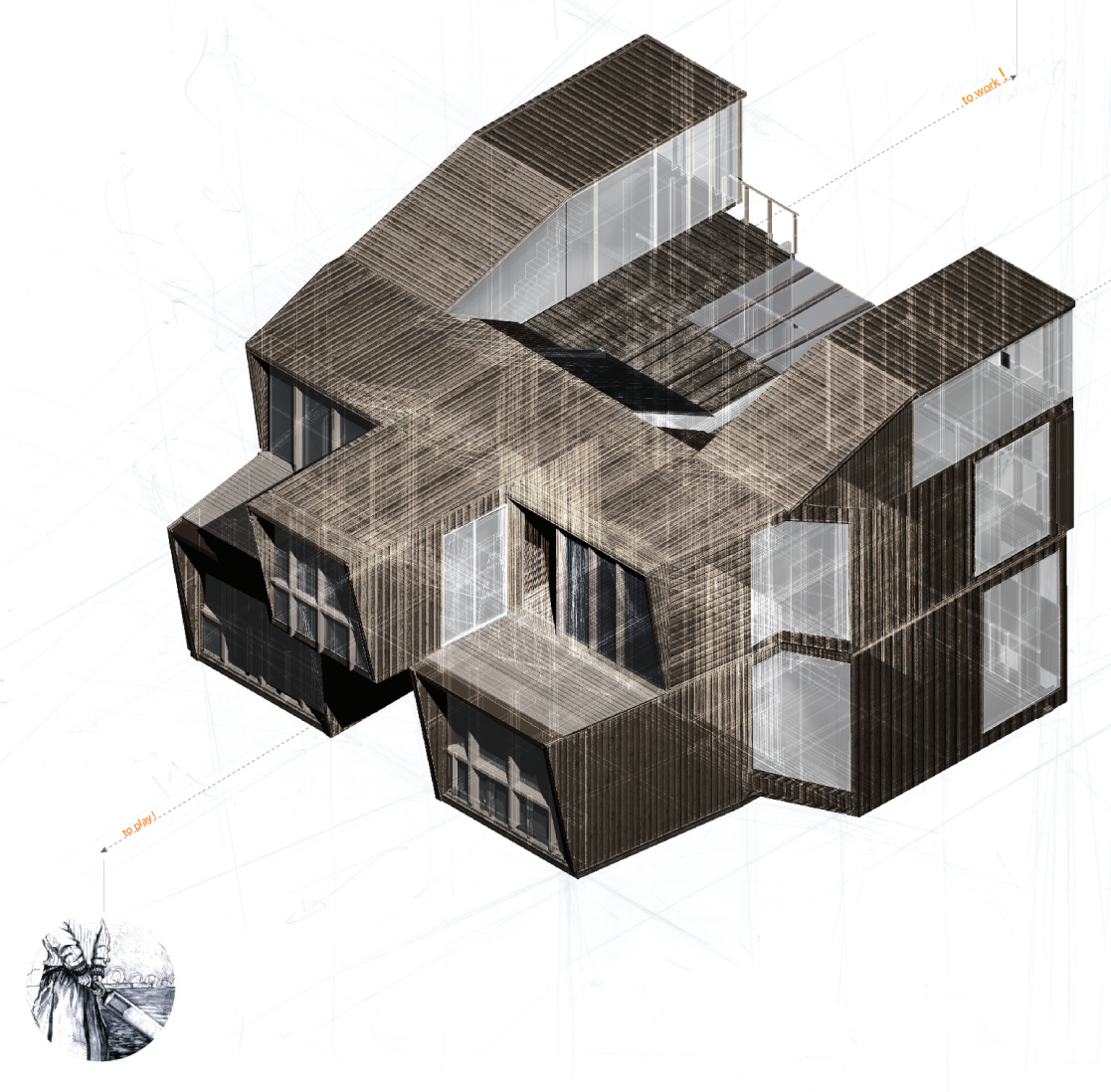

Figure 62 : Far left: plans one housing 'cluster', with two studio aparmetn on the lower floor and two 2-bedroom apartments spread over the upper two floors.

Figure 63 : Above: the housing 'cluster' showing the arrangement of the four apartments forming shared and private spaces.

With the eight 'clusters' of housing along the length of each pavilion, notions of shared, densified living environments are introduced.

Each of these clusters contains four apartments, which share an entrance/ lobby off the chasm. The configuration of the apartments allows moments of shared living, but retains the privacy of the residents. (see figures 62-63) 


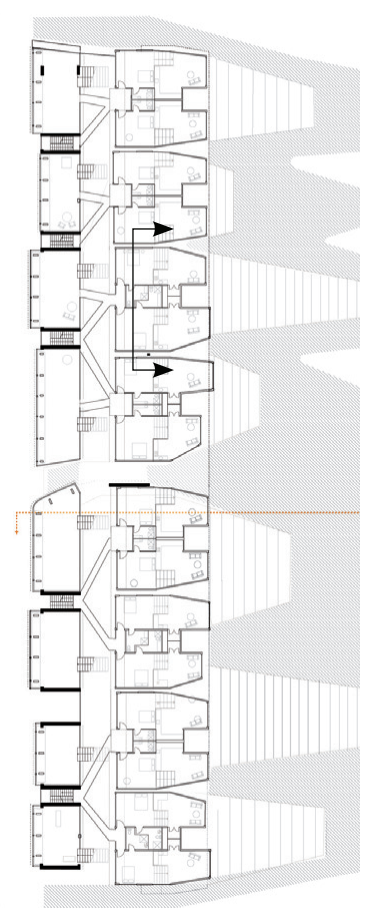

The living spaces that are created are dense but private; individualised but connected. As the four apartments within each cluster look onto a central entrance way, and with offices just moments away (directly across the chasm), this close proximity invites new creative and social dialogues to form.

Beneath these housing clusters, the public porch spans as an open expansive void; and an array of commercial, leisure and communal activities unfold. With this rich mixture of private, social and public activities taking place over its five floors, the building is microcosm of the transforming suburb.

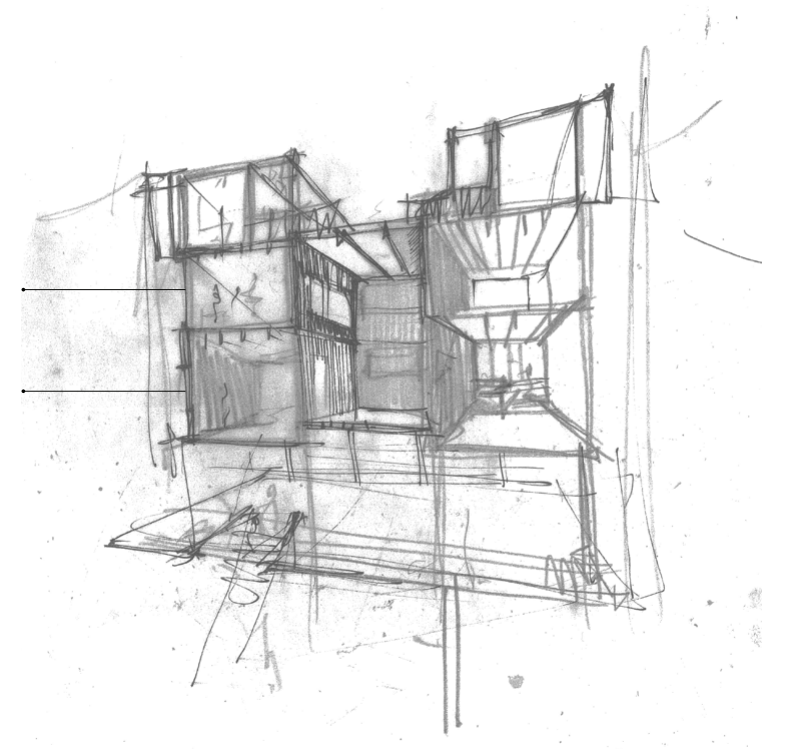

Figure 64 : Right: a partial section through one housing 'cluster' and the public/commercial spaces

$<$ Figure 65 : Left: a sketch of this section. 


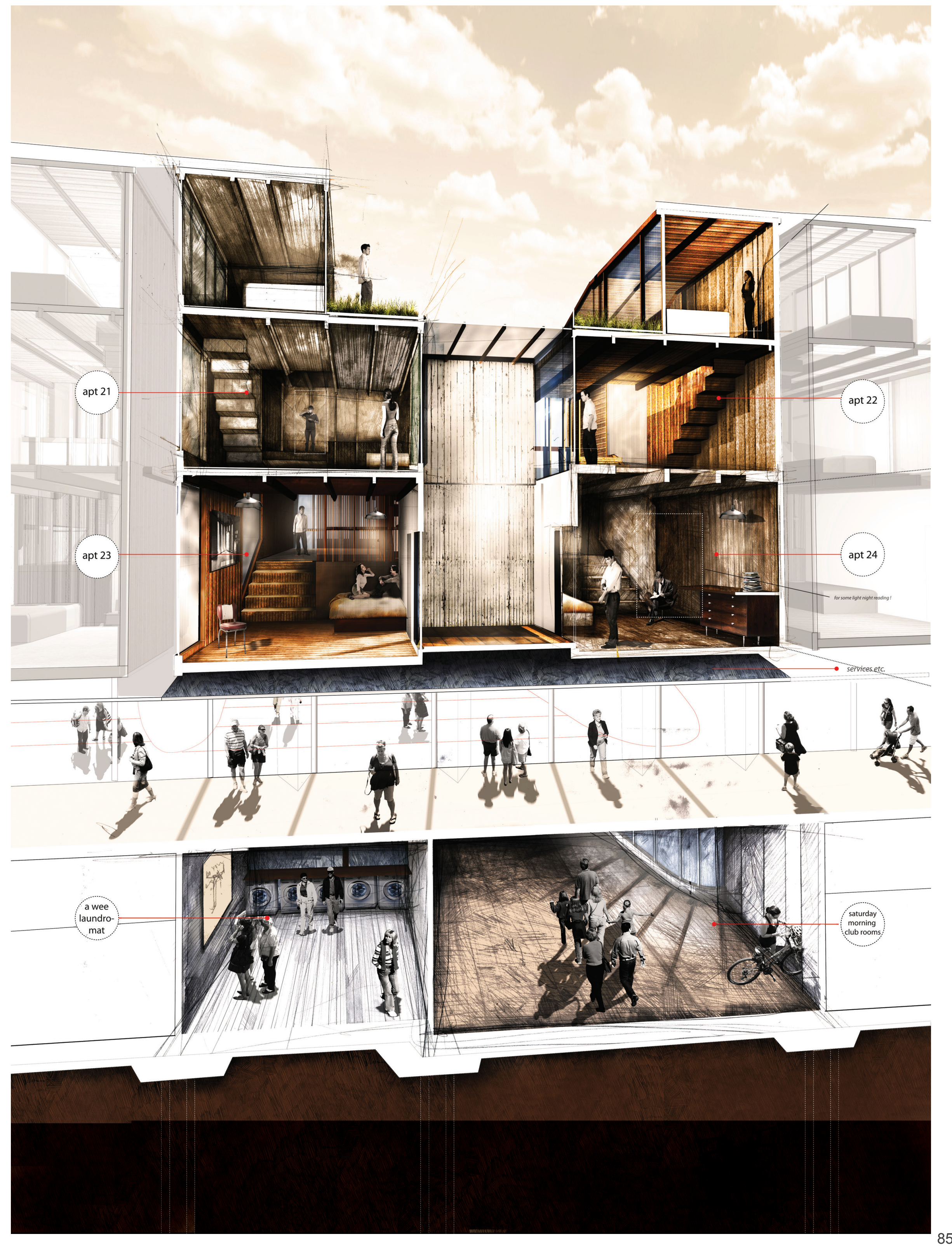



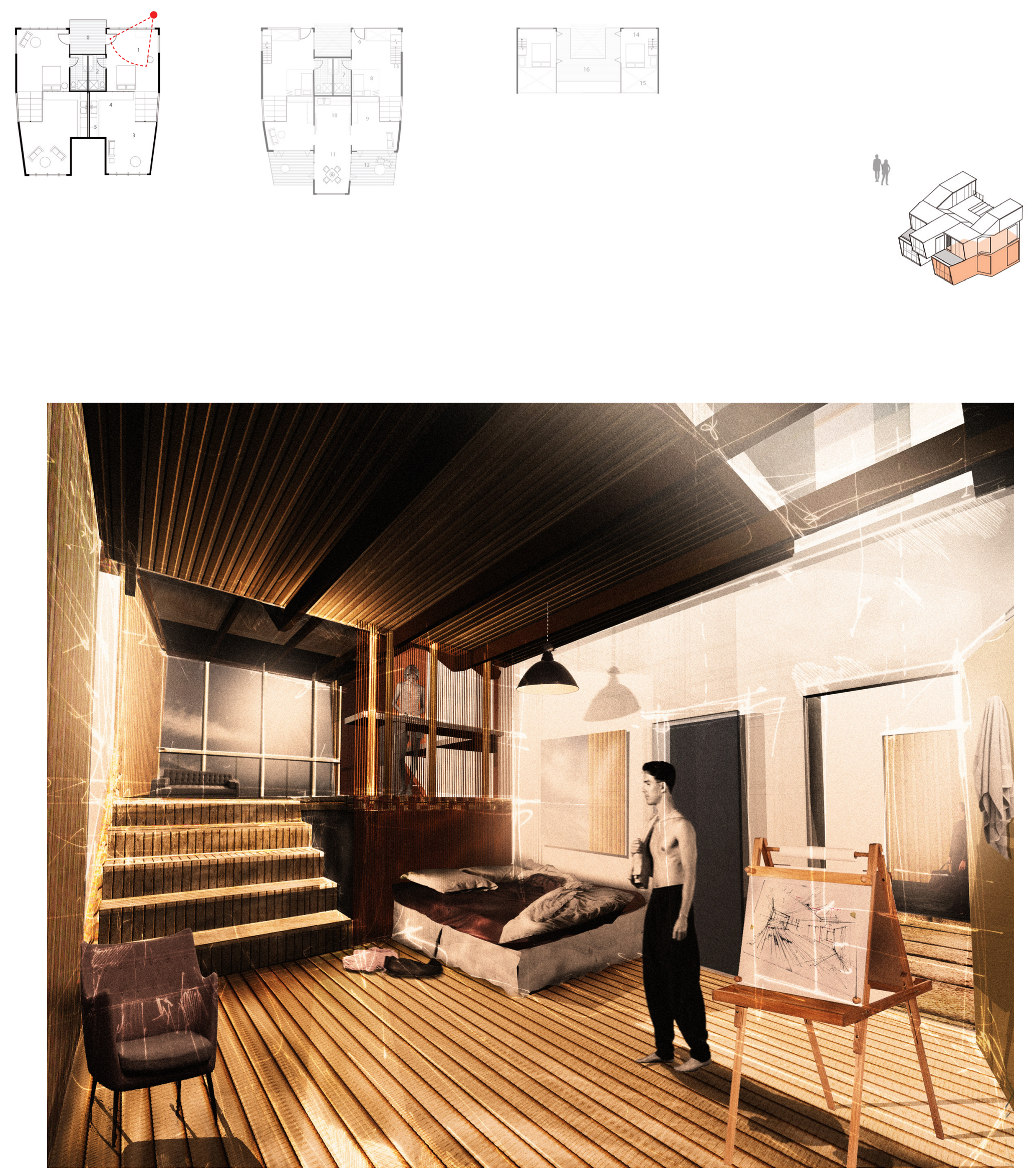
Figure 66 : Above: A bed-sit/studio apartment looking
out towards the park. Stairs up into the lounge form a fundamental relationship to the external form of the 

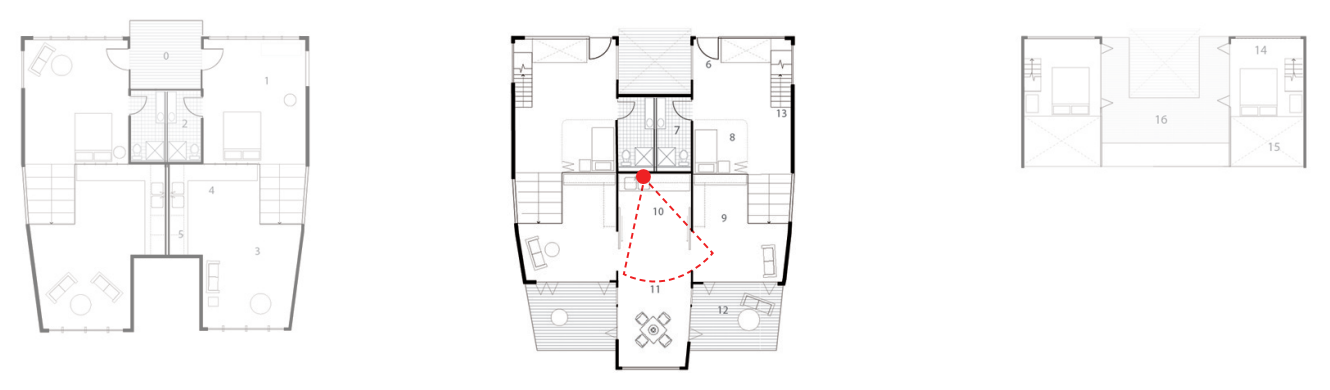

Will
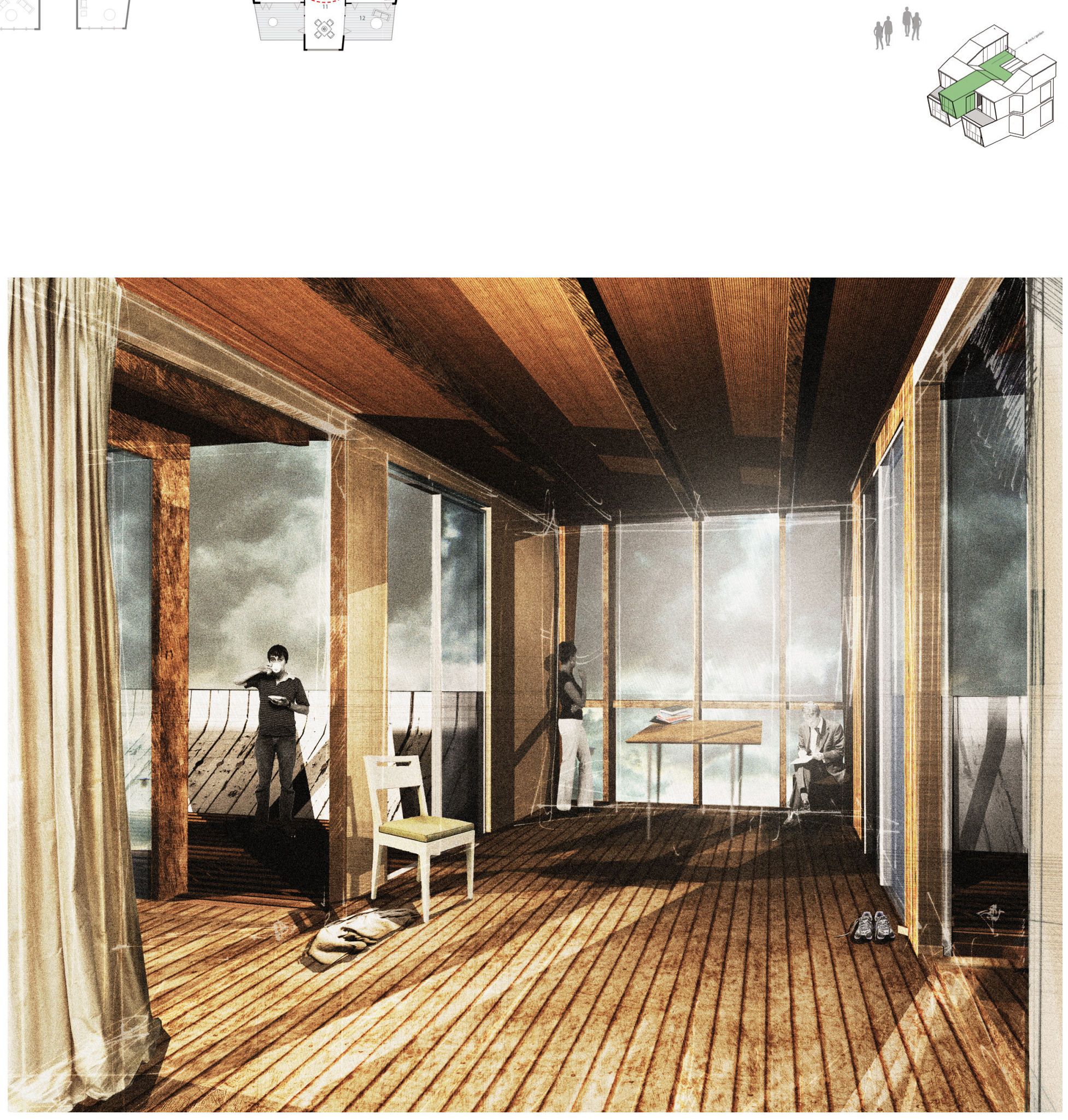

Figure 67 : Looking out from the shared living space of the
upper apartments: with balconies on either side of a central upper apartments: with balconies on either side of a centration
protruding space which punctures through the pavilion structure. 

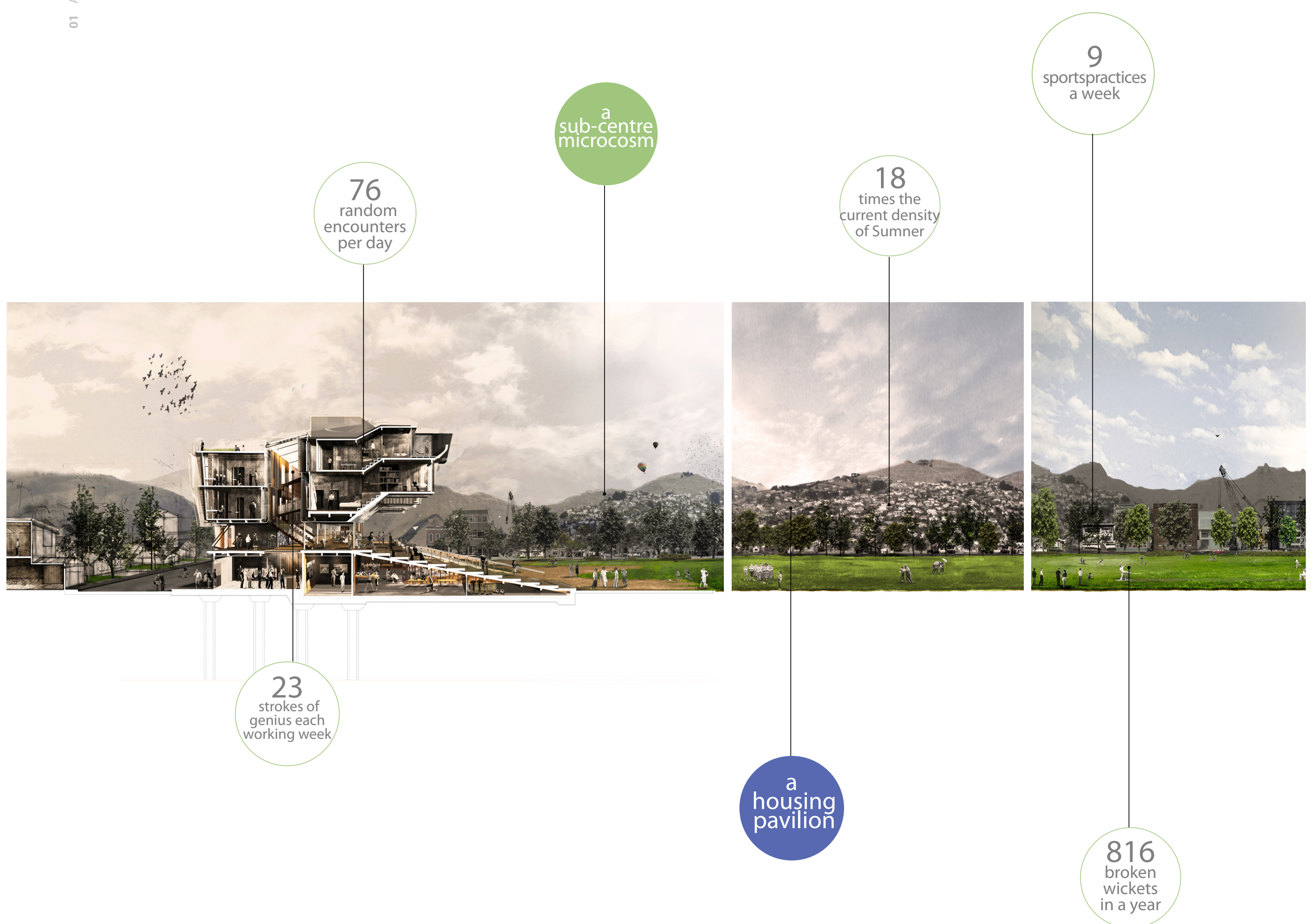


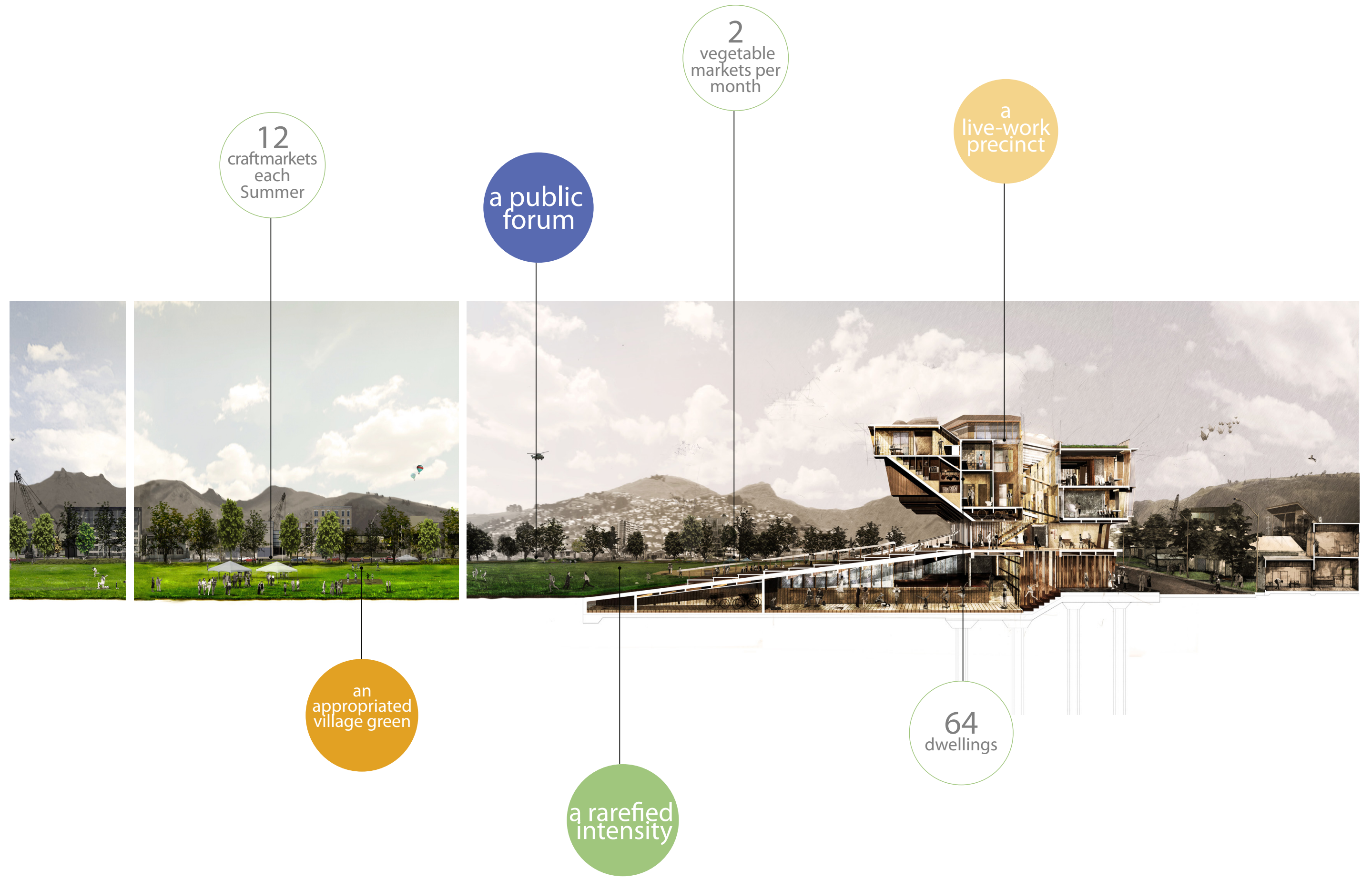
Figure 68 : Longitudinal section suggesting the multiple
ways in which the scheme may be understood. 


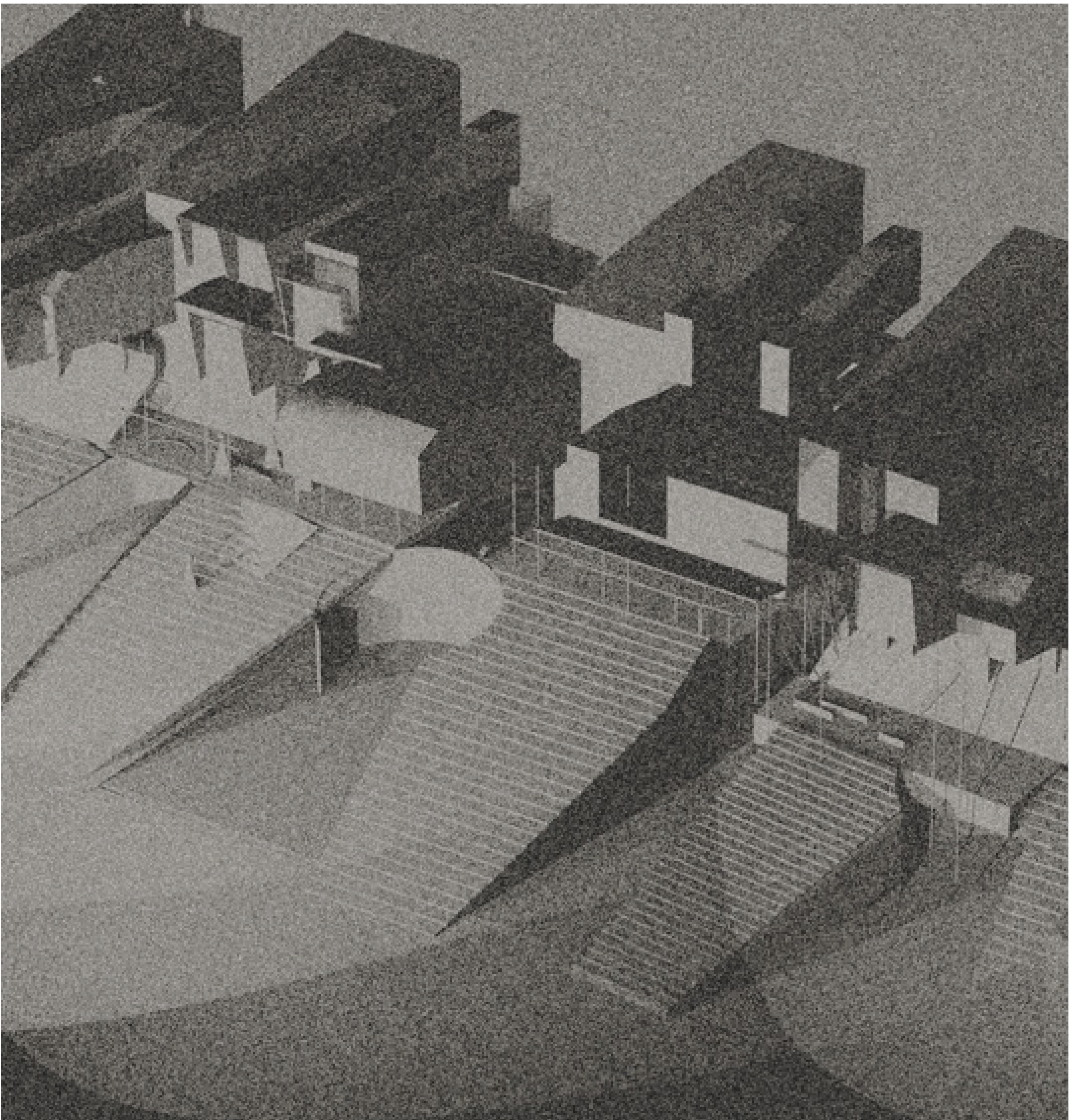

Figure 1: Perspsective of early design iteration, from above. 


\section{Paper}

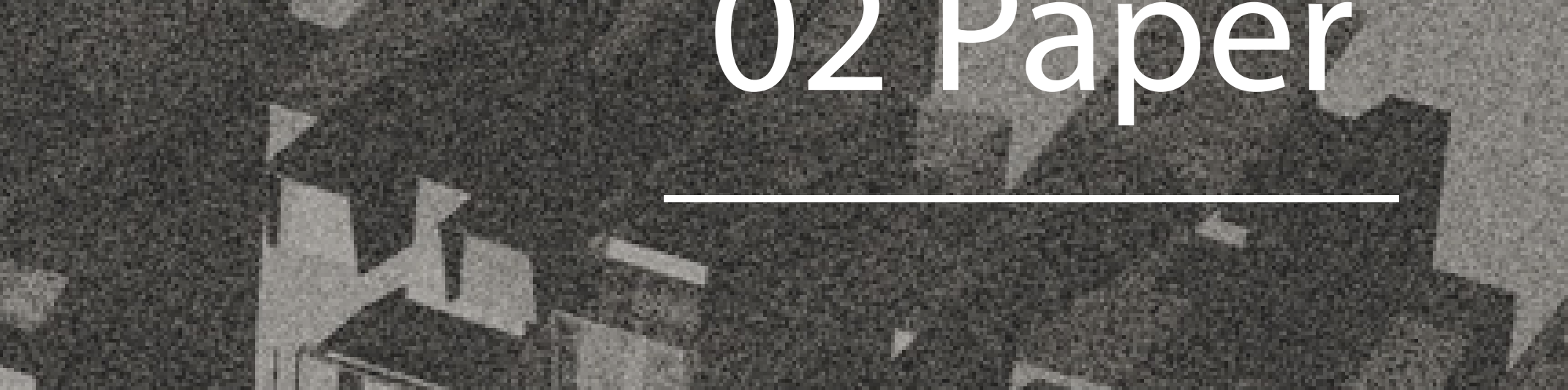

Towards the Sub-Centre as Architecture's C. $x=2$ Project
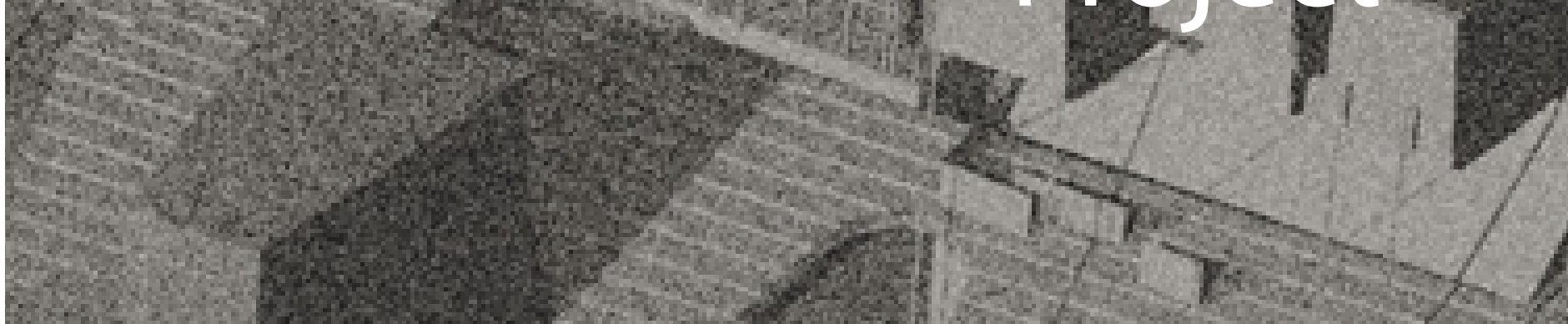
"However a minor role that architects may now have in shaping the global metropolis or dystopian urban vision, they remain smitten with the city"

- Helen Castle, 2012

- Dana Cuff and Roger Sherman, 2011

Significant urban transformations have often triggered investigations into the possibility of architecture's urban instrumentality. Here, the basic understanding that architecture as an urban constituent contributes to a political, social or cultural understanding of the city, has given rise to the conviction that architecture plays a critical role in progressing influencing and producing the city - and simply, cannot be separated from it. Bound by the potential of doing something not just in but to the city, architecture has considered the city its foremost motivation, provocation or project - and has attempted to actively position itself in relation to it. In this way, the idea of the city - an understanding with which architecture may actively endeavour to engage - has occupied a firm place within architectural thought.

Perplexedly, as we enter an urban century par excellence, the notion that architecture may have some instrumental role in

shaping the city, or an understanding thereof, has significantly diminished. As architecture busies itself with an array of more 'imperative' economic and ecological concerns, engaging the city as an idea invariably plagued by social, cultural and political concepts has been relegated to the back-end of a long list of priorities. Meanwhile, the city itself has actively receded as an architectural focal point: invariably becoming the territory of other domains - planning, infrastructure, landscape, information and data - and apparently no longer requiring architecture as it once did'. In turn, the possibility of actively and productively relating to the city - construing it indeed as architecture's project - has been dismissed as a vacuous promise, dispelled as a myth, and reserved for the indulgent.

While today, the role of architecture as urban form is often discussed - exhaustively, even - in relation to the city's more intangible and internal factors, it is seen to have a less-thaninstrumental role on the city itself. Insofar as architecture and the city are understood to be further apart than ever, a state of neglect has become the status quo: while architecture is in the city at an unprecedented rate; it is no longer of the city.

Though the effects of architecture's urban acrimony and ambivalence are variously attested to and widely lamented ${ }^{2}$, they have a particular resonance in the emergent subcentre of the polycentric city: an urban nomenclature bearing current relevance to a New Zealand context, yet also being proliferated at a universal scale. As an urban phenomenon the sub-centre is quite uncanny - neither periphery nor centre; neither autonomous nor subsumed; both concentrated and dispersed; and slung between the urban and suburban - but not quite either. Appearing in suburbs, hinterlands, brownfield sites or urban peripheries as 'partial' alternatives to the city-centre, these urban contexts are invariably linked to an array of diverse cultural, political and social changes. Yet, disproportionately plagued by economic imperatives, and

\footnotetext{
As Helen Castle suggests $(2012,5)$

2 (Cuff and Sherman 2011) (Eisenschmidt 2012), amongst others, attest to this consequences of this state of neglect
} 
materialising at an often-rapid rate, emergent sub-centres frequently attest to a much narrower set of concerns: in a way that sees them regularly lack the cultural, critical mass required to assert their role, and indeed effective centrality, within a larger polycentric system. This is a perilous projection for cities with a CBD, let alone without - as is effectively the case within the city of Christchurch. Here, it seems, there is a real provocation to re-visit architecture's urban instrumentality.

This research papers sets out with two basic agendas: firstly, to expand on the sub-centre as an urban context desperately requiring architecture's urban instrumentality; and secondly, to explore how architecture might engage the sub-centre as its project .

Part one - "A Cry for Attention" - establishes the sub-centre as a necessary project for architecture. First outlining the subcentre as a distinct urban condition, it discusses how current, narrow conceptions have had a disproportionate bearing on the sub-centre, with resounding and often detrimental effects. Responding to calls for an expanded conception of the sub-centre - indeed, as a context as politically, socially and culturally significant as it is economically - I argue that architecture should be considered as a necessary and critical sub-centre medium. In this way, it is imperative for architecture to actively seek new means and modalities to engage the subcentre as a distinct urban condition.

Part two - "Towards the Sub-centre as Architecture's Project" - explores how recent ideas about architecture's urban instrumentality may be mobilised in a sub-centre context, ultimately seeking terms of engagement through which architecture might actively engage the sub-centre as its project. After briefly outlining a recent state of neglect between architecture and the city, it outlines contemporary efforts toward a renewed architectural instrumentality in the city, largely through architectural form. Two positions are negotiated here: one of formal resistance, the other of radicalised, contextual opportunism. Through a dialectical synthesis, these approaches are seen to offer a broad set of 'strategies' for architecture to actively engage the subcentre. Particularly, these strategies enable architecture to simultaneously take a position on the sub-centre through separate, absolute form - and take a position in it, by exploiting the formal opportunities of this emergent context. This is not intended as a set of objective 'rules' - but a broad possible architectural response to an urban context that is, indeed, begging for its attention.
With its underlying problem-solution format in tow, this paper channels an urban predicament through an architectural problem, and then funnels an architectural strategy back out as urban potential - indeed, holding the urban problem as inextricable from the architectural exploration it propels. In this way, the conviction at the root of the paper is enacted and exacted: a flickering between the city (here, the sub-centre) and architecture as intertwined conditions - indeed, not as separate concerns, but in constant, productive and evernecessary dialogue. 


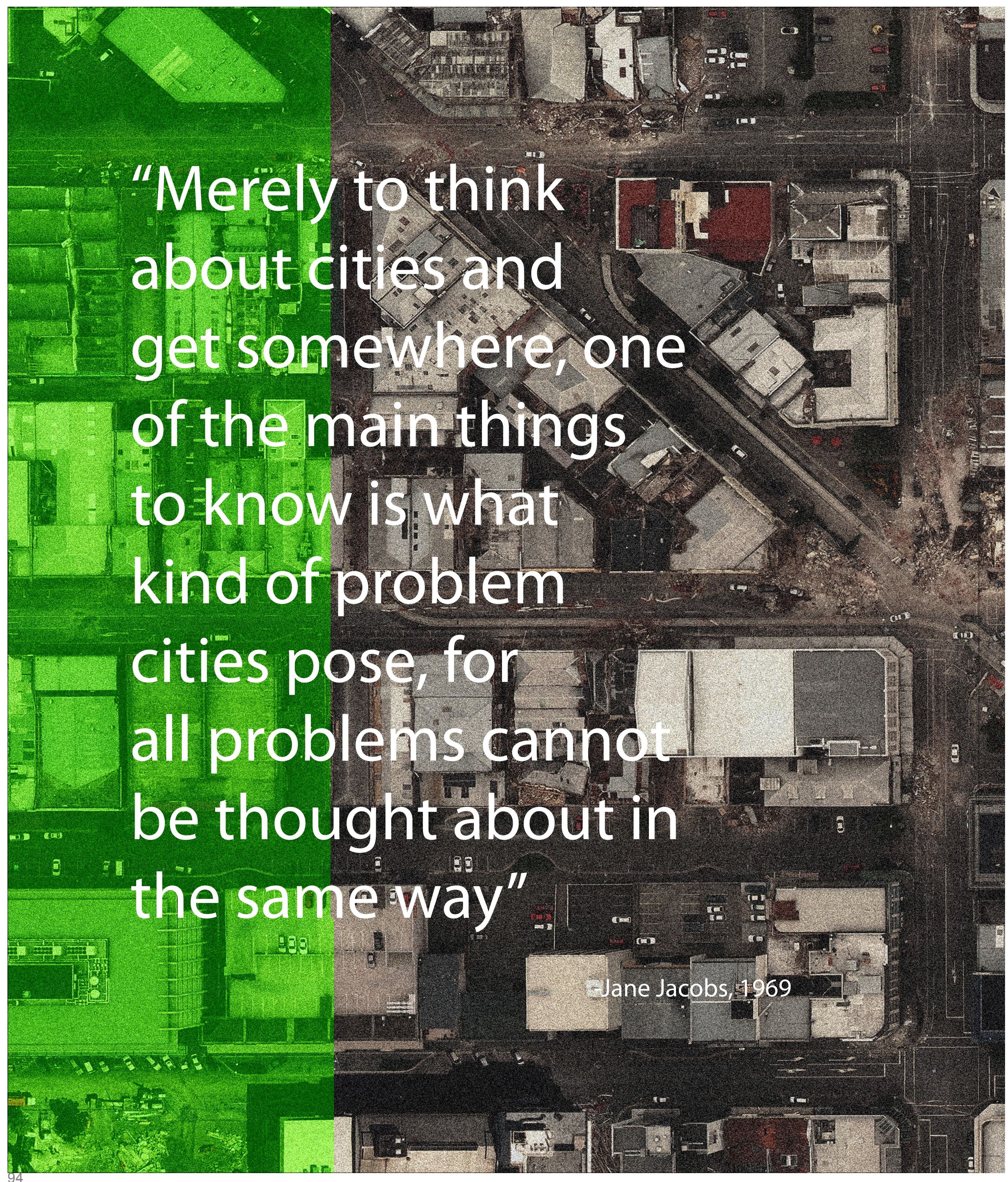




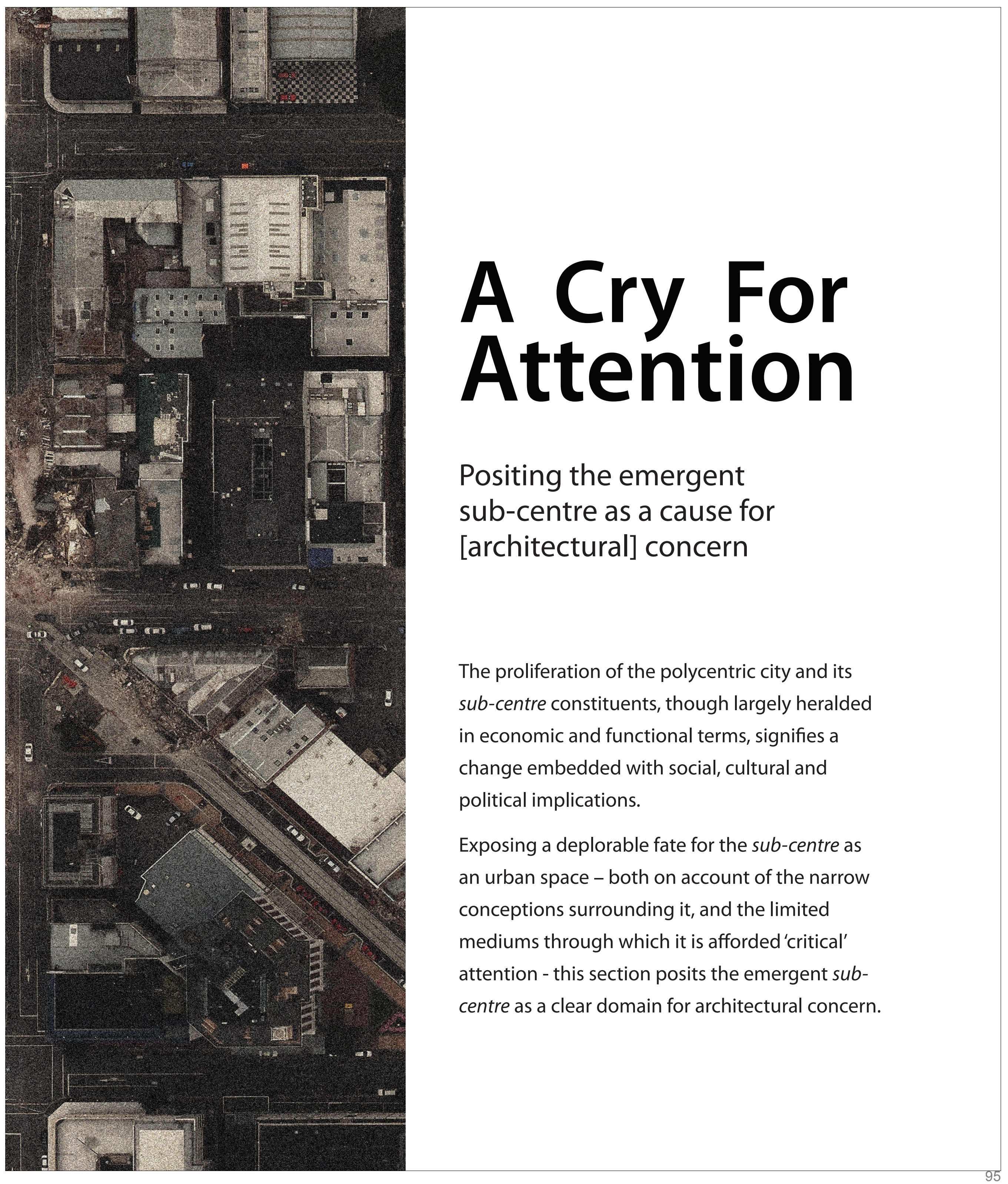




\subsection{The Proliferation of a Polycentric Urban Culture: Towards a changing idea of the city}

"We cannot continue to flirt with the romantic idea that everything can be squeezed in one single centre"

- Steffen Lehmann, 2010

"It appears that the twenty-first century city is a city of paradox: It has points of intense concentration whilst also being dispersed, forming a low-density city in a high-density (data) landscape."

- John Worthington, 2009

No marshalling of statistics is necessary to note that urban regions, globally, have entered a new, major phase of spatial and organisational development: one effectively arising from the convergence of centralising and decentralising forces which have characterised the history of urbanisation to date ${ }^{2}$. Though often described as a defining characteristic of the 21 st century city, the polycentric configuration has been transforming and re-structuring urban regions around the world for decades: installing an ever-familiar mélange of networks, flows, and urbanised peripheries, while rendering the long-prevailing monocentric model (a singular city centre and 'its' peripheral suburbs) increasingly an object of nostalgia ${ }^{3}$.
Today this multi-centred model holds an unprecedented place in urban thought: the term polycentric no longer confined to cities such as Tokyo, Los Angeles or Berlin, but increasingly and frequently employed in relation to cities as disparate in scale, location, character and population as Shanghai, Singapore, and Stockholm - and indeed, as antipodean as Christchurch ${ }^{4}$. Likewise, in a discursive context, the growing prevalence of this pluri-centred urban structure has procured a string of attempts to measure and understand its significance and potentials: in a way that has seen the terms megalopolis, megacity or metacity and network-city - each manifestations of a multi-centred urban phenomena - emerge as a ubiquitous part of urban-related vocabulary (gradually supplanting metropolis, city, and town $n^{5}$ ).

Notably, the ubiquity of this urban model is far from fortuitous. Rather, while the onset of polycentrism is attributed - in its initial stages - to external or uncontrollable forces ${ }^{6}$, active attempts to reinforce or generate polycentricity have permeated urban policies in recent years. This reflects the widely-held view that a shift toward a multi-centred model is not only a necessary progression in many cases, but moreover, offers a diverse range of opportunities to the 21 st century urban environment. Indeed, to this end, a polycentric configuration has often been touted as panacea for socio-economic and ecological urban problems ${ }^{7}$.

Yet, despite the polyvalent advantages of a 'perfect' polycentric urbanism, there are also wide-ranging challenges attached to its effective, strategic implementation - particularly on account of the rate and scale at which it is being developed, and the complex, paradoxical forces that characterise $i^{8}$. Steffen Lehmann, a practicing academic specializing in developing urban polycentricity, summarises the lamentable environments that may result:

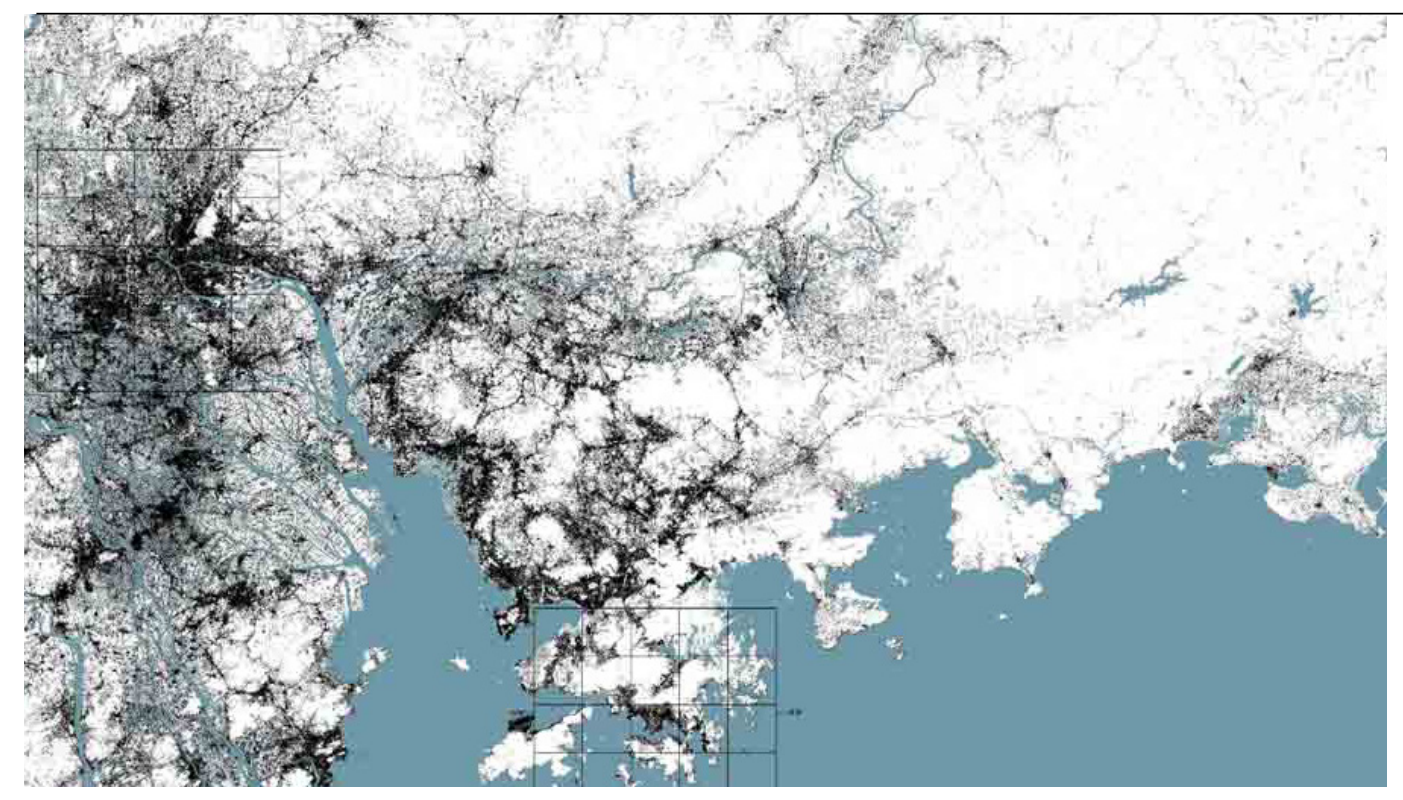

Figure 3: Pearl River Delta Urban

Agglomeration. This multi-centred region in China, comprising five functionally and conomically linked cities was the subject of OMA's inflential study 'Great Leap Forwa OMA's influentia study'Great Leap Forwa (2002). As a polycentric model has proliferated on a global scale - whether at the scale of the city or the region (megalopolis) - studies into multi-centred configurations have become increasingly important.

\footnotetext{
93

2 Polycentricity is also explained to arise from the conflation of 'global'/

'local', or 'expanding'//intensifying', distributed/intensifying forces, or centripetal and centrifugal forces. (Hall and Pain 2006) (Jaume 2011) (Lehmann 2010) (Muñiz, Garcia-López and Galindo 2008)
} 3 (Hall and Pain, 2006).

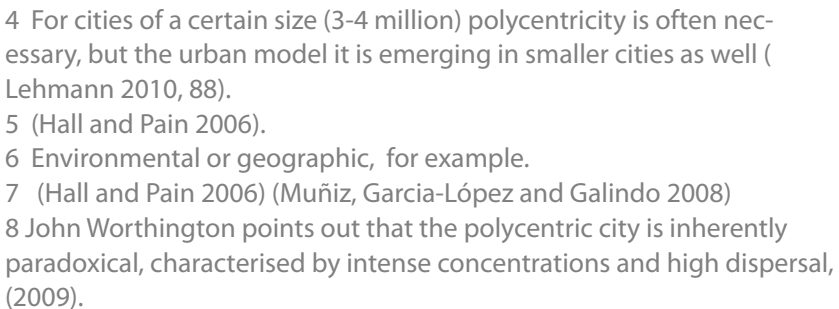
ssary, but the urban model it is emerging in smaller cities as well Lehmann 2010, 88)

(Hall and Pain 2006) (Muñiz, Garcia-López and Galindo 2008) paradoxical characterised by intense concentrations and high dispersal 2009) 
...despite the polyvalent advantages of a 'perfect' polycentric urbanism, there are also wide-ranging challenges attached to its effective, strategic implementation

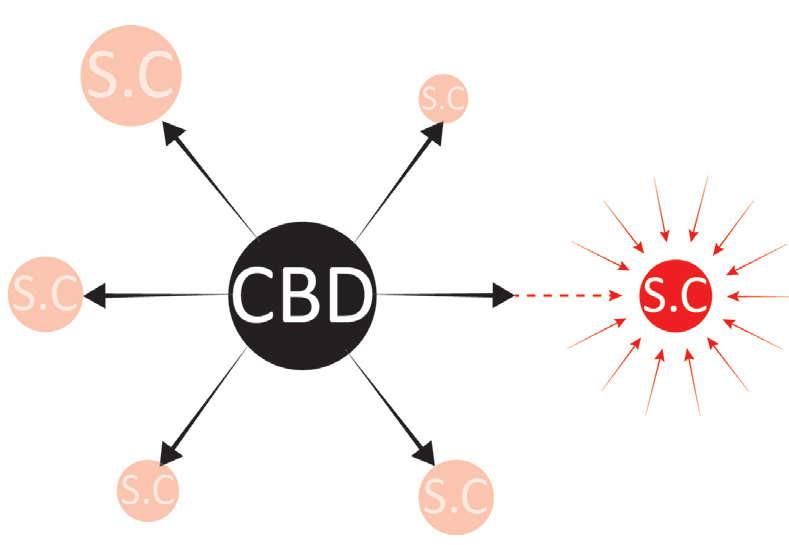

Figure 4: Sub-centre forces. The emergence of the sub-centre can be understood according to parallel, paradoxical forces: decentralization at a regional scale (as employment and densities disperse from the city-centre) and centralization at a local scale (as the city-centre) and centralization at a local scale (as
urban functions gravitate towards peripheries and urban functions gravitate towards peripheries and
previously non-urban sites, to forming dense livework clusters that counter urban sprawl). "a dramatically forced urbanization [...] without any urban qualities [...] without good public spaces, one-dimensional, lacking the rich complexity, which cities always require to have to be vibrant."

Indeed, rife with both uncharted opportunities and challenges, emergent polycentric cities have increasingly been regarded as 'urban laboratories' in recent years, serving as the focus for a wealth of diverse research ${ }^{10}$. (fig. 3) Moreover, while efforts to divulge the implications of this urban paradigm - through individual and often disparate examples - have often borne insights, a more in-depth understanding as well as effective models of 'best practice' remain widely sought.

\subsection{A Not So Peripheral Reality: the (sub) centre of our attention}

"...the urban field as an archipelago of enclaves"

Steffen Lehmann, 2010

Dealing critically with a polycentric model, the rise of the urban sub-centre as a structuring element of urban regions becomes fundamental. Variously described as an alternative city-centre, a 'city within the city' or an 'edge-city', the sub-centre is a definitive part of the strategy to impose an inter-connected, pluri-centric structure on expanding urban regions, through

$9(2010,94)$

10 OMA's study of the China's Pearl River Delta Region in China in 2002, which studied economic and trade-based potentials of polycentric condition is a particularly seminal example in this regard. See Great Leap Forward (OMA, 2002)

1195 a network of diverse, functionally-connected, concentrated nodes or distributed densities. Seen to benefit both from the economic and cultural advantages available in a large metropolis, while maintaining its local independence, the subcentre has emerged as "a strategy to upgrade, diversity and increase the attractiveness of the metropolis"12, and as a mode of sustainable urban-renewal: indeed, actively resisting the effects of decentralized, and unstructured and 'infinite' sprawl. In this regard the sub-centre is lauded as a positive urban phenomenon, extolled especially for its socio-economic and ecological benefits.

There are two points duly-noted about these urban spaces before proceeding to a more critical discussion. Firstly, on account of the simultaneous decentralizing and centralizing forces directing its emergence, the sub-centre is generally premised on a two-fold condition. On the one hand, it is understood to operate as a 'partial'13 alternative to the original and often-shrinking, city-centre - in terms of employment, density, multi-functionality, for example - in a way that renders it largely autonomous (certainly, more so than the suburb). On the other hand, it is predicated on being functionally-connected to other proximate (sub)centres, and thus necessarily operates as a structural element of a larger urban system rather than as an independent 'city' or 'centre' on its own'14. (fig. 4). This underlying condition - of integration and separation - is characterised by Potsdamer Platz (Berlin) and La Defense (Paris): each commercially-driven, financial districts that, while operating as an autonomous urban districts in one sense, are indelibly part of a larger urban structure. (fig. 5-6, overleaf)

2 (Lehmann 2010 88-89). See also (Jaume 2011)(Dear 2011, 226) 13 I use the term partial here in relation to the idea that as the polycentric urban model gradually subsumes a monocentric one, the former singular' city centre is effectively dispersed around multiple sub-centres (which, in this way, form a collective alternative to the CBD). 14 While the size of the sub-centre may vary greatly, these two conditions may be seen as relatively constant. 

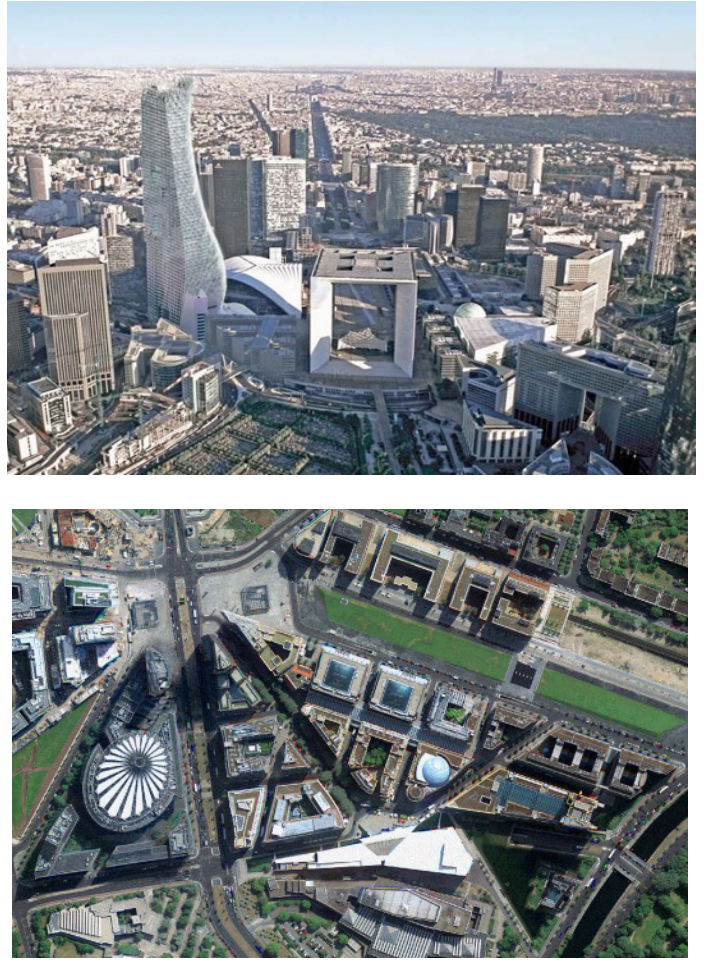

Figures 5-6: City within the city: Urban subcentres generally subscribe to a two-part condition autonomous and clearly-defined within an urban region on one hand; functionally-integrated on the other. Shown here: La Defense, a purpose built sub-centre in Paris, France (above) and Potsdamer Platz, Berlin.

Secondly, it is notable that sub-centre is typically characterised by a rapid 'speed of delivery', on account of the economic motivations typically propelling its emergence. Potsdamer Platz, for example, transformed a virtually empty part of Berlin into a populous centre in the short timeframe of 1994-2000; and likewise, new sub-centres in China have 'sprung up' recently at a "breath-taking" rate ${ }^{15}$. To this end, the sub-centre does not emerge organically or as a traditional city-centres would - and may more accurately be described as 'instant' urban environments ${ }^{\prime 16}$. As Lehmann writes in this regard:

"The scale and pace of change and the level of ambition [...] are quite extraordinary."17

\subsection{The Sub-Centre as a Curiously Imageable Urban Phenomenon}

In this paper, I am not concerned with specific sub-centre examples nor their widely-heralded potentials, so much as two interrelated aspects: firstly, the degree to which the sub-centre may be considered a generic urban condition; secondly, the impact that this may have on the development of these urban

\footnotetext{
15 (Lehmann 2010, 89). (Muñiz, Garcia-López, \& Galindo, 2008) 16 (Lehmann 2010, 92). Of course the sub-centre is a much more complex and diverse urban concept than I have explained here. However, across the many definitions afforded to these new urban spaces, these conditions appear to be fairly consistent - despite other variances such as scale, density, for example.

17 (Lehmann 2010, 93)
}

environments, given their often-rapid materialisation. Indeed, beyond the to-be-expected deviations in practice, sub-centres can be seen to betray common characteristics: inter alia, an overt economic impetus; a certain degree of autonomy; and a necessary level of urban integration. What appears to be afforded by these commonalties is a pervasive image of the sub-centre: not only evident but effective in the way this urban condition is discussed, depicted - and this paper argues designed and developed ${ }^{18}$. This salient 'imageability' of the subcentre can be observed on three interrelated counts.

\section{A Limited Discourse}

Firstly, the pervasiveness of this image may be seen in the limited manner in which the sub-centre is discussed, and in turn, defined. Current discourse largely attends to the economic, functional, ecological, demographic and transit-based implications of this emergent urban space ${ }^{19}$ : even when greater socio-cultural or political issues are at stake, and despite the fact that these emerging urban environments often comprise living and public functions as well as working and commercial - which certainly seems to warrant a wider discussion ${ }^{20}$. While the sub-centre is inseparable from a host of broader structural changes - new patterns of activity and mobility; re-configured decision-making bodies; new social formations, to name but a few - popular definitions afford a far narrower and invariably more prosaic understanding of this urban space ${ }^{21}$. The description of the sub-centre as a "precisely functional machine ${ }^{\prime 22}$ is exemplary here: testament to the prevalence of economic and functional concerns in its conception, and likewise, the ready neglect of its wider significance as an urban space (and, indeed place).

\section{Sub-centre as 'Urban Artefact'}

Secondly, this image-based reading of the sub-centre is evident in its description as an urban artefact, presenting a 'once in a lifetime' and 'extraordinary' opportunity to re-think urban ideals. ${ }^{23}$ This object-based perception is indelibly linked to the propensity for sub-centres to emerge as newly-fabricated and fully-designed environments ${ }^{24}$, and likewise, in the depiction of sub-centres as singular urban 'objects' (figs. 7-8). To this

\footnotetext{
18 The notion of the urban image has often been discussed in urbanrelated discourse, and will be expanded on later in this paper. 19 See, (McMillen, Parr 2004)(Lehmann 2010, 30) (Cladera, Duarte and Moix 2009)(Jaume 2011) (Vasanen, 2012).

20 While sub-centres often comprise living as well as working environments, the latter is normallover-emphasised meaning ronments, the latteris nomaly factors take precedence their definition, and in turn, their development (Jaume 2011).

21 (Thun, Velikov and Ripley 2012) (Vasanen, 2012) (Jaume 2011) outline the broader structural changes to which the sub-centre is linked and suggest that models based purely on employment density evade the'reality' of the sub-centre as a complex, cultural urban space . 22 (Lehmann 2010, 94)

23 (Lehmann 2010 8)

24 New sub-centres are often led by competition design, and are developer-driven: both of which may allow them to be constructed acdeveloper-driven: both of which may allow them to be constructed ac cording to a singular, overarching vision.
} 
end, these new urban spaces are readily reduced to a single overarching idea; concept, vision, or function, and in some instances, even referred to as an urban product ${ }^{25}$.

\section{Conceptual Simplicity (an object of comparison)}

Thirdly, the existence the sub-centre image is evident in current modes of critique, reflection and comparison to which these urban spaces are subject. Here, the conceptual simplicity afforded by a narrowly-focused image permits existing sub-centres in China, Germany, France to be compared and contrasted under a common heading (or as a common urban type)-despite their overt, and in many ways stifling, differences: not only physically, but politically, socially, culturally (to name but a brief few) ${ }^{26}$.

\subsection{An Urban Image - But to What End?}

On each of these counts, the sub-centre, with its salient image in tow, presents an urban condition clearly distinct from the city at large - indeed, seldom afforded such a singular or 'objectified' classification ${ }^{27}$. This provokes a compelling question as to whether - or to what degree - the narrow and potent image of the sub-centre, championing it indeed as a functional artefact, may influence the rapid-production of these actual urban spaces $^{28}$.

Despite its generality, this need not be a question far-fetched. The relationship between the image and generation of urban places has often been deliberated in recent years. Frank Eckardt and Peter Kreisl - professors in Urban Sociology and Land-use Planning, respectively - have identified for example that the formation of conceptual urban'images'has been common and in fact quite necessary in recent decades of urban transformation ${ }^{29}$.

The sub-centre, with its salient image in tow, presents an urban condition clearly distinct from the city at large - indeed, seldom afforded such a singular or 'objectified' classification.

\footnotetext{
5 In China, sub-centres are advertised in brochures, with market jargon in tact, and often 'branded' by a dominant function (Lehmann, 2010, p. 88)

26 See (Lehmann 2010).

27 Peter Trummer argues that complexity and growth of contemporary urbanism has largely undermined 'the city' being read as an object (2013, p. 52).

28 Though not discussed in this way, this image-based-ontology is complicit within the concerns many researchers, whom have noted a prevailing tendency to conceive the sub-centre strictly on economic, demographic, functional and ecological terms, and expressed unease over the implications this may have for emergent or future sub-centres. See, for example, (Jaume 2011) (Lehmann 2010) (Gyurkovich 2011) 29 (2004, 13-15)
}
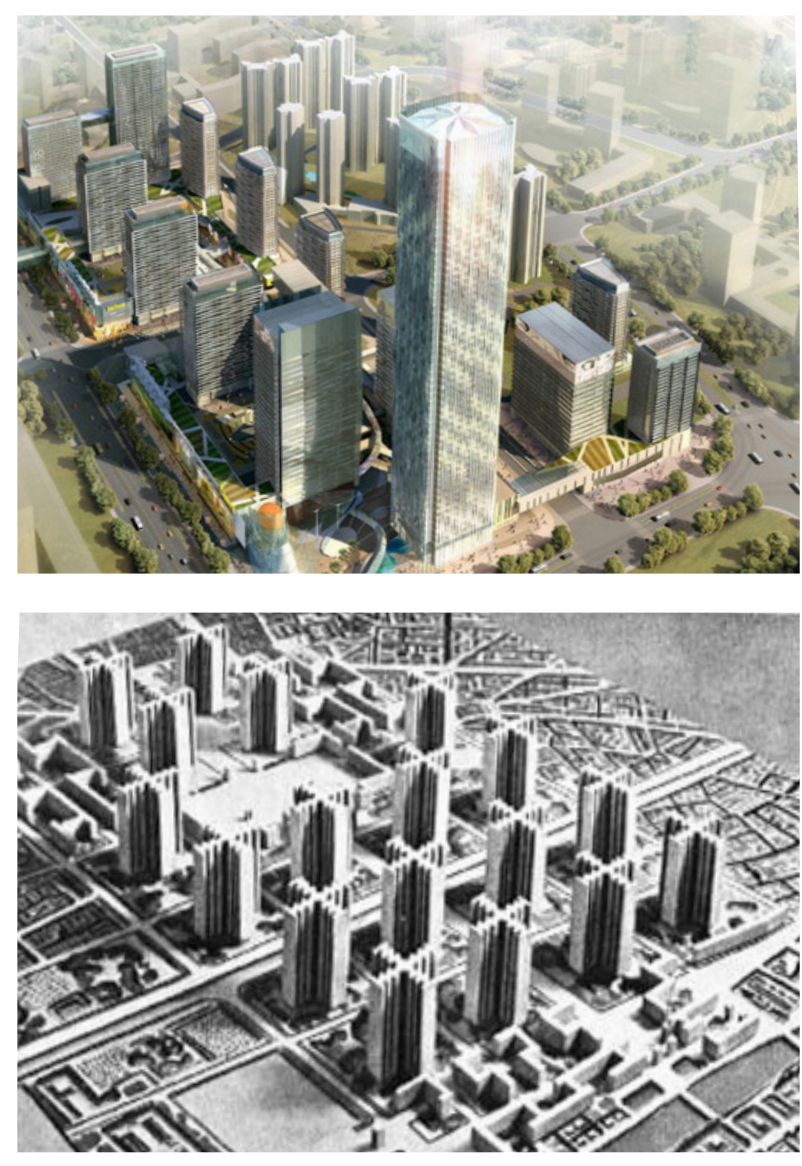

Figures 7-8: The Image of the Sub-centr Described as 'instant 'cities, emergent sub-centres bear some resemblance to the 'objectified' projects of modern urban planning - as fully-designed environments depicted in a single image. Above, the Zhenru Sub-centre in Shanghai, China (under construction) compared to Le Corbusier's Plan Voisin for Paris (1925): both total designed 'cities', yet the former is being realised.

Moreover, these authors suggest that these urban 'images' have often exercised a 'guiding' role in the objectives and generation of urbanism: to the extent that images of urbanity may indeed become self-fulfilling prophesies ${ }^{30}$. Similarly, Brendan Cormier argues that descriptions of urbanity and actual urbanisms are in a normative, self-perpetuating and cyclical relationship, meaning the former may become inscribed in the material spatial, formal and cultural environments of the latter (and vice versa) ${ }^{31}$.

To this end - and especially given its swift emergence and oftentimes, lack of pre-existing context ${ }^{32}$ - it seems quite probable that the image afforded to the sub-centre may indeed yield a salient influence on its material constitution.

30 Ibic

31 Cormier contends that the relationship here is far from passive: "urbanism has both a descriptive and a normative side - meaning our theories of how a city ought to exist often become inscribed in the future city, and thus affect anew our descriptive theory" $(2012,5)$.

32 As Lehmann identifies, sub-centres cannot evolve as traditional city centres, but rather, at a fast rate or as 'instant environments' $(2010,94)$ Emerging on greenfield, brownfield and peripheral urban sites (in addition to urban infill sites), sub-centres may have little cultural or 'physical' context to respond to. 


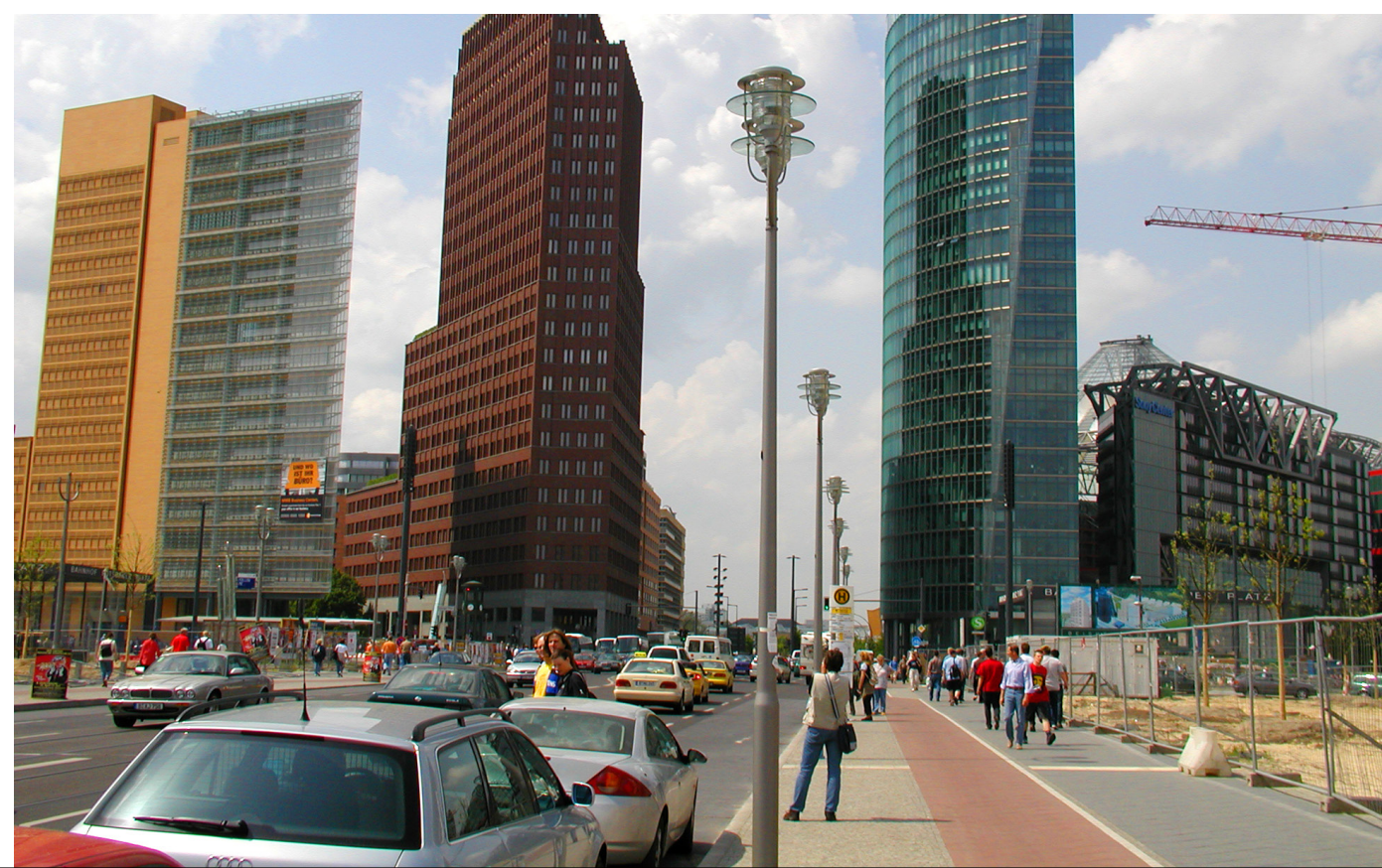

Figure 9: An Image reified. Reviews of Potsdamer Platz have labelled it as the 'Americanization of the New Berlin', with large-scale commercialised buildings and 'streets that look like shopping malls'. These reviews allude
sholings and 'streets that look like to a lack of cultural and physical authenticity, and appear to arise on account of the overt economic imperatives, which are imbued in the built fabric of this rapidly-constructed urban centre.

\subsection{An Image Brought to Bear: like-minded intitiatives}

The image of the sub-centre outlined above seems to have indeed had a lucid - or even guiding ${ }^{33}$ - impact on objectives and initiatives to develop, demarcate and identify emergent sub-centres, in many instances. These generative strategies are often noted for their uncompromising attendance to economic and functional concerns - employment densities, for example - while consideration of factors beyond these concerns may be lacking: the sub-centre's political distinction within the wider polycentric region, or its spatial interaction with its adjacent hinterland ${ }^{34}$.

The apprehension here is quite straightforward: while these narrow-focused initiatives may deliver the functional, economic, and even demographic ambitions of emergent sub-centres - satisfying their existence as'functional machines' par excellence - their quality and effectiveness as vital urban places within a larger urban system may be impeded ${ }^{35}$. Typifying this sentiment, Mateusz Gyurkovich in his PhD on Eastern European sub-centres contends that:

"attempts to demarcate new sub-centres based upon economic, demographic and transport premises only passing over [other] principles [...] jeopardize [the effectiveness of their urbanity]"36

\footnotetext{
33 As asserted to often be the case by Eckardt and Kreisl $(2004,13)$ 34 For example, initiatives to identify emergent sub-centres in Barcelona utilised measurements of employment population density and mobility flows, without taking their spatial interaction with adjacen hinterland into account (Cladera, Duarte \& Moix 2009, 2831-2845) (Kloosterman and Musterd 2001, 623-632)

35 Cladera, Duarte and Moix's paper "Urban Structure and Polycentrism: Towards a Redefinition of the Sub-centre Concept" (2009) suggests that without addressing their spatial interaction with adjacent hinterland a criticlosectivess in the harer urban context - sub-centres may emerge as disconnec larger uran context - sub-centes may emerge as disconnected and/ or dysfunctional in the polycentric region. See also (Jaume, 2011) (Gyurkovich, 2011) (Bureau Stedelijke Planning: General Information, 2012)

36 (Gyurkovich 2011)
}

Similarly, Steffan Lehmann suggests that on account of the limited conception and thusly-orientated strategies afforded to them, rapidly-materialising sub-centres may emerge as:

"often uniform in their offering... to maximize the customer experience... a reflection of the experience economy [...] with their focus on efficiency, functionality and convenience ${ }^{\prime \prime 37}$

\subsection{An Image Manifest: instant city/peripheral fix}

"Now, not only small communities are resorts, but also entire world cities, are constructed according to a formula..."

- Keller Easterling, $2012^{38}$

In practice, the correlation between this narrowly-focused urban image and the sub-centre's material constitution is often quite transparent. Berlin's Potsdamer Platz is exemplary in this regard (fig. 9). This high-functioning business district has been cited as Berlin's answer to Las Vegas; A "New Manhattanism"; "The Americanization of the New Berlin"; the-shopping mall-city ${ }^{39}$. These reviews broadly allude to a lack of physical and cultural authenticity in this fast-constructed urban centre, largely on account of the salient economic imperatives which have induced a highlycommodified built environment. Indeed, notwithstanding Potsdamer Platz's vast commercial success, a pervasive image of economic efficiency and functional autonomy appears to have stifled its broader success as an urban place: preventing it from transcending its immediate role as a commercial and business centre. In short, deviating little from the ambitions

ann $2010,9.94$

$38 \quad 59$

39 (Ward 2006, 83)(Strom 2001, 199) 
propelling it, Potsdamer Platz's built environment invites a similarly-narrow urban identity (and despite, intriguingly, its salient political underpinnings ${ }^{40}$ ) .

At a different end of the spectrum, though not dissimilarly, Shanghai's Chongqing sub-centre has been apprehended for its "disneyesque" and"themed" nature ${ }^{41}$. Here, the decision to'apply' a function to this new urban district as a means to 'differentiate' it and imbue it with a distinct identity is exemplary of its underlying imperatives. Reflecting this functional uniformity in its totality, Chongqing comprises a homogenized, uniform urban environment, with little affectation for a cultural identity, sense of place, or authenticity. (figs. 10-11)

Further afield, the logical limits of this function-based approach may be seen in emergent sub-centres that are literally "developer driven" brands or urban products, published in brochures with market jargon intact ${ }^{42}$. Though on the one hand, these extreme tendencies may be culturally-inflected (as such more likely to take place in the East), on the other, emergent sub-centres are regularly and widely described to wear their economic imperatives on their sleeve - indeed, suggesting these proclivities are somewhat transcendent. To this effect, new and emergent sub-centres are commonly cited as " market driven rather than user-led", consumer commodities, "over functionalised" , "lacking authenticity" and "super functional, themed locations" ${ }^{43}$.

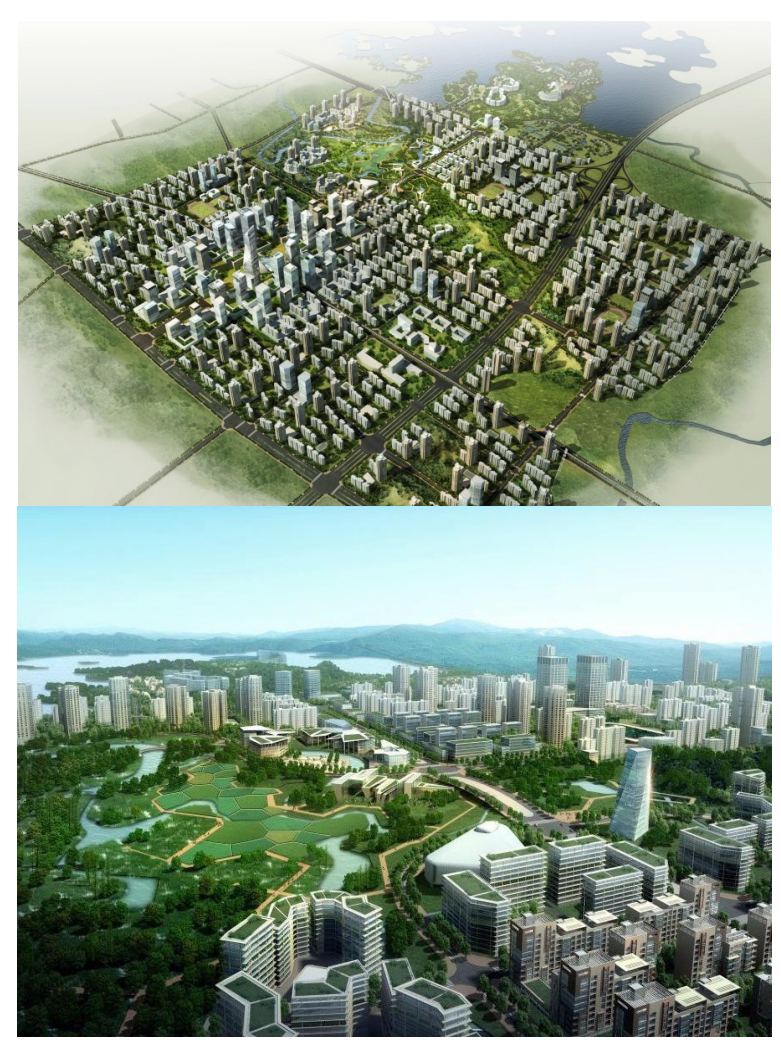

\subsection{An Image Reified}

Through these specific and more general reviews, sub-centres cross-continent-may be seen to betray a compelling consistency. Be it through the shopping-mall streets of Potsdamer Platz or the sheer function-based monotony of modernist urban forms in Chongqing, economic and functional imperatives pervade the material, visual and formal environments of these urban contexts: indeed, often playing a guiding role in their resultant urbanity. Moreover, without here broaching the relationship between urban form and the life it engenders, these attributes largely correlate with the cultural character of emergent sub-centres: likewise perceived to be uniform, commoditized, homogeneous, and somehow, plagued with an air of disingenuity. David Smiley and Mark Robbins perceptively summarise the resulting dilemma:

"the dilemma is that these instant environments [...] have frequently lost the chance encounters and unplanned authenticity of the experience they are aiming to create.... the qualities... the public culture" ${ }^{44}$

40 Potsdamer Platz is particularly interesting because it was underwritten by clear political and cultural histories, yet, economic imperatives pervade it emergence as a sub-centre and indeed, characterise it as a uniform business district (and little else).

41 (Lehmann 2010, 94)

42 Zhenru Sub-Centre, for example, is marketed as an ecological city:

"a Green Business District in compliance with world-class green construction criteria" (Lehmann 2010, 93)

43 See Worthington (2009) Hajer and Reijndorp, 2004, in: (Lehmann

2010, 94-95), for example.

44 (2002) as cited by Lehmann $(2010,94$

Though on the one hand, these extreme tendencies may be culturally-inflected [...] on the other, emergent sub-centres are regularly and widely described to wear their economic imperatives on their sleeve.
Figure 10-11: The image of transit-oriented development and functional efficiency. The Chongqing sub-centre in China is seen to "[integrate] the surrounding industrial developments with major functional clusters". The urban environment consists of a commodified, commercialized and homogenized urban morphology, that while rich in "sustainable design elements" qualitatively reinforces an 'image' of the subcentre as principled by economic, functional, and transit-orientated concerns. (Arup, 2012) and transit-orientated concerns. (Arup, 2012). sub-centres often materialise as such. 


\subsection{A Beleaguered City, Before it has even Begun}

"Urbanism has both a descriptive and a normative side - meaning our theories of how a city ought to exist often become inscribed in the future city, and thus affect anew our descriptive theory"

Brendan Cormier, $2012^{45}$

Without suggesting a strictly causal or scientific relationship between the image of the sub-centre and the urban spaces (and places) produced under its name, it seems reasonable to infer that there is an evident correlation between the two ${ }^{46}$. Likewise, the understanding that urban generation may "concretize the objectives $^{\prime \prime 4}$ of a dominant urban vision appears to have some tangible implications for these new urban centres - especially given their often-rapid emergence. As a fitting analogy, it may be seen that where the sprawling suburbanization of the sixties onwards emanated from an image of "integrated individualisation and communitarian lifestyles" ${ }^{\prime \prime 8}$, the genesis of sub-centre stems from an image of economic efficiency and functional autonomy - and broadly speaking, often lends itself to a physical and cultural environment oriented as such.

Taken seriously, these somewhat-formulaic tendencies pose a deplorable fate for emergent sub-centres generally - which, it seems, may easily fall short of the vibrant, social and cultural urban places, living (and working) centres, and veritable citycentre alternatives they are often required to be ${ }^{49}$. Broadly speaking, and returning to Lehmann's earlier assertion, these tendencies are wont to create a lamentable polycentric reality:

" a dramatically forced urbanization of landscape without any urban qualities, cities without good public spaces, onedimensional, lacking the rich complexity, which cities always require to have to be vibrant." 50

Taken seriously, these somewhat formulaic tendencies pose a deplorable fate for emergent sub-centres generally - which, it seems, may easily fall short of the vibrant, social and cultural urban places, living (and working) centres, and veritable city-centre alternatives they are often required to be.

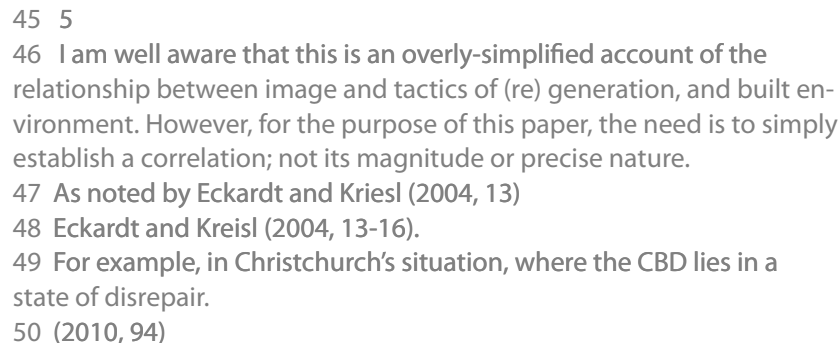

This tightly-bound relationship between what is essentially the thinking about and making of the sub-centre, invokes two equally interrelated responses. Firstly, it outlines the need for an expanded conception (or image) of the sub-centre, addressing its broader significance as an urban space, beyond economic and functional concerns. Secondly, it summons a consideration of the mediums, modes of production and strategies through which it the sub-centre is 'made' or generated - and its current image so diligently brought to bear ${ }^{51}$. Given their strong correlation as such, it seems any effort to foreground qualities of public culture, authentic experience and chance encounter in emergent sub-centres must regard these two components as integrally linked ${ }^{52}$.

\subsection{Delimiting the Image: towards a cultural conception of the sub-centre through four ideas.}

"Urban life is wider, broader, more coloured, complex and even paradoxical than can be captured by one single image"

- Frank Eckardt and Peter Kreisl 2004, 16

The first of these objectives - an 'expanded conception' of the sub-centre - has been called for by numerous researchers. While some literally outline the need for "a re-definition of the sub-centre concept" beyond economic-density and transitrelated issues, for example, more broadly there is an emphasis on critically re-formulating ideas and widening discussions around the sub-centre, such as to enable its development as an authentic, meaningful and invariably complex urban place ${ }^{53}$.

Though a re-definition is well beyond the scope - and point - of this paper, there are nevertheless a number of key points crucial to a broader cultural, social and political understanding of this emergent urban space, and which appear to have often been neglected or supressed by current definitions. In this way, rather than posit an idea of the sub-centre, I will summarize four ideas of the sub-centre which underlie its broader significance as an urban space, and in this way, are integral to any expanded conception $^{54}$

\footnotetext{
51 Despite the somewhat ambitious undertones of these objectives, 'failing' urban images have been actively addressed and 'expanded' in the past as a means to re-orientate successful urban development. See (Eckardt \& Kreisl, 2004, pp. 13-16). Certainly, the call for urban (re) generation to include a broader array of practices and disciplines is common.

52 I would argue that the peculiar conditions of the sub-centre that alins it to be this image can be seen to have been brought to bea this image can be seen to have been brought to bear on the material constitution of the sub-centre at present, suggest that approaching this image-genesis model critically (and addressing both aspects simultaneously) is a logical progression.

53 (Cladera, Duarte and Moix 2009, 2841 ). See also (Jaume 2011) (Dear 2011, 226-228).

54 I use this term as a comparison to the sub-centre image described above, and to refer a broader, multi-facetted, qualitative and more ideological reading of the sub-centre.
} 


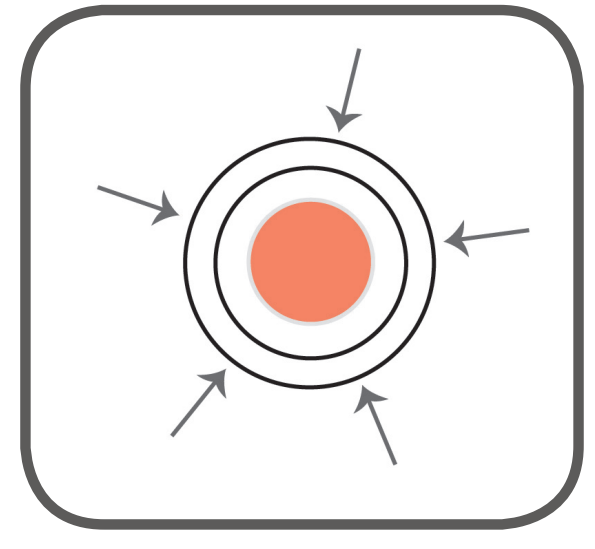

Figure 12: The Sub-centre as a vertiable condition of centrality

\section{IDEA \#1: A (sub) version of the centre: rebranding a cultural obsession:}

\section{A veritable condition of centrality}

The first of these arises from the underlying logic of the polycentric configuration as a network of separate, interconnected centres. For the sub-centre itself, the significance of this hierarchical shift lies in its competing role with the social-cultural 'obsession' that is the city-centre ${ }^{55}$. Simply, insofar as the city-centre may be considered a social, political and cultural phenomenon, it follows that the sub-centre, as a vying alternative, must attempt to distinguish itself on those same terms, rather than just those economic or functional, demographic ${ }^{56}$. To this end, the sub-centre must aspire to the qualities somewhat objectively associated with the city-centre as an idea: inter alia, gravity, potency, vitality, density and attractiveness. ${ }^{57}$. While these are by no means easy feats - and indeed, the notion of the city-centre is increasingly blurred and hard to define, let alone create - foregoing the this aspect of the sub-centre severely jeopardizes its emergence as a successful public urban space. ${ }^{58}$ (fig. 12)

The need for an 'holistic' centrality is confounded by Lehmann's assertion that the term sub-cluster may be more appropriate than sub-centre. Here, he suggests a graduated, monocentric internal hierarchy (from centre to periphery) has little relevance to new sub-centres and indicates a more uniform condition

\footnotetext{
55 (Gyurkovich, 2011) insists that any conception of the sub-centre must'start' with the idea of a city'centre'

56 Steffen Lehmann argues that the sub-centre must be specially branded in order to distinguish itself from the historical city-core: indicating that the sub-centre be identifiable not only as separate from the city centre but, operate as an alternative to it, performing a comparable role $(2010,95)$

57 Especially where sub-centres comprise living and public cultures (as well as working), this gravity and potency is necessarily social, cultural, political rather than just economic - bringing into play not only density and function - but an array of factors indelibly linked to this type of trac tion: aesthesis; history; context; composition; attractiveness, legibility, aesthesis, richness, social equity, vitality, attachment, identity, scale to name but a few(Gyurkovich 2011) (Cladera, Duarte, \& Moix, 2009)
} $58 \quad$ (Gyurkovich, 2011) of centrality is more probable ${ }^{59}$. This invites a paradoxical idea: an imbued condition of centrality that permeates and characterises this sub-centre in its entirety, differentiating it from the decentralized space beyond it, and insodoing reinforcing the logic of the polycentric city. ${ }^{60}$

\section{IDEA \#2: The sub-centre as fabricated urbanity}

\section{An embodied transition}

The second of these ideas arise from the fact that, unlike the organic, gradual processes informing and forming traditional city centres, the sub-centre as a concept involves the imposition of urbanity at a fast or even "forced" rate ${ }^{61}$. Complicit in this rapid rate-of-change is a series of significant shifts: from low to high density; from private to public; from single to multifunctioning; from residential to commercial; from slow to fast pace; from distributed to concentrated; and often, from periphery to centre. Broadly speaking, these shifts impart a state of urbanity to previously non-urban (or significantly less-urban) contexts, inducing cultures of density, intensity, diversity, fast-pace and congestion, for example. (fig. 13). The significance here lies in the necessary transition in order to create these conditions in the sub-centre: the idea that this urbanity is invariably fabricated or forged. Indeed, though often framed in a functional terms, this transition - corresponds with a number of significant changes that are invariably cultural, political, societal and social in nature. As Lehmann writes:

"These [sub-centre] agglomerations correspond to new social formations in a very direct manner, providing habitation for a more and more individualized, inter-connected society"62

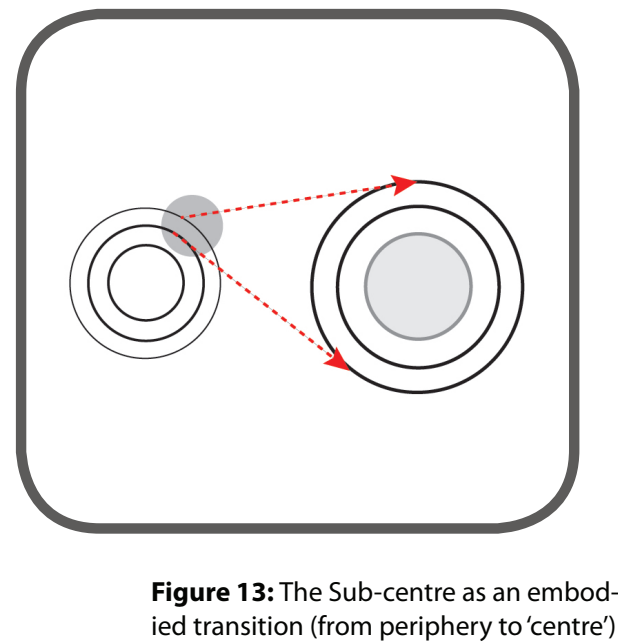

is a more traditional monocentric understanding of centre (2010)

60 This binary relationship between the centralized and decentralized areas of the polycentric city underlies its broader logic and distinguishes it from the sprawling urban regions it is predicated on replacing.

61 (Lehmann 2010)

62 (Lehmann 2010, 94) 
On all of these counts, this fabrication of urbanity constitutes a critical component of the idea of the sub-centre: not only in terms of enabling an 'appropriate' urbanity for the sub-centre relative to its pre-existing context - but moreover, as means of mitigating the potentially negative effects of a forced or abrupt state of urbanity (which may induce both a lack of contextualism and authenticity, as seen in the earlier examples).

\section{IDEA \#3: The sub-centre as one of many}

A themed centre or a differentiated centrality?

The basic significance of the sub-centre as a centre - outlined above - is confounded by the fact that it is necessarily one of many centres: none of which should, in theory, be leading ${ }^{63}$. This leads to the somewhat paradoxical notion that while it is $a$ centre, it is not the centre. There is two primary implications for the sub-centre here: on the one hand it must be distinct from nearby centres - functionally, politically, spatially and culturally - in order to establish its independence within the polycentric region ${ }^{64}$. On the other, its interaction with these centres is essential for the effectiveness of the larger urban system ${ }^{65}$. This need to both differentiate from, and identify with is fundamental, and suggests the sub-centre's identity must critically operate across multiple scales. Moreover, to avoid the 'functional-identity' this may amount to - tantamount to the crudely themed sub-centre environments described earlier - this idea should characterise the sub-centre holistically: necessitating differentiation and integration that spans social, political and cultural dimensions ${ }^{66}$. (fig. 14)

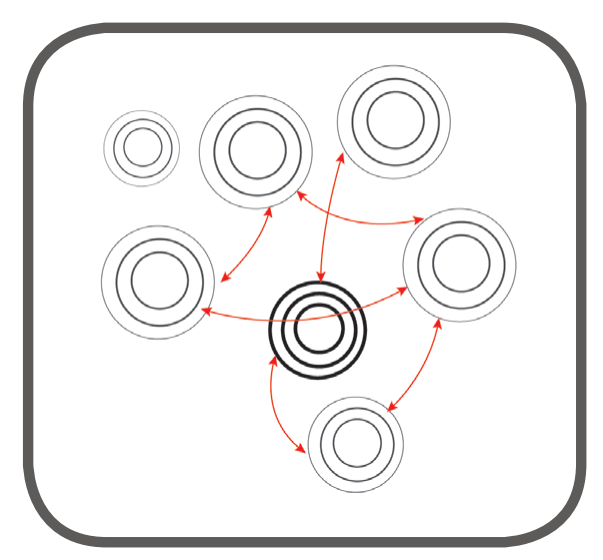

Fig ure 14: the Sub-centre as one of many centres

63 (Kloosterman and Musterd 2001, 623-632)

64 Kloosterman and Musterd for example, stress that sub-centres must be'politically and spatially distinct' from nearby centres. (2001, 623-632)

65 (Cladera, Duarte and Moix 2009) . For example, to enable its nonhierarchical functioning.

66 With Shanghai's Zhenru Sub-Centre, it was seen that for the larger polycentric model to work effectively, the sub-centre must differentiate itself from nearby sub-centres. In this case, a technological, industrial, logistic focus enabled this differentiation - a clearly 'functional' approach to identity. (Lehmann 2010, 93).

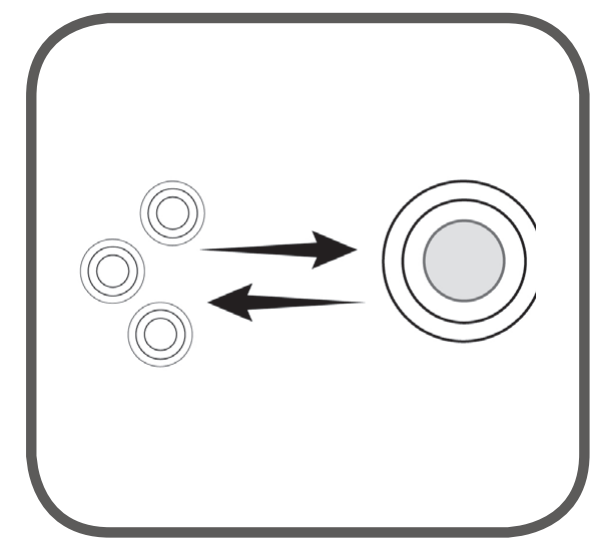

Figure 15: the Sub-centre as imbued with paradox and opportunities as part of a larger urban system

\section{IDEA \#4: The Sub-centre as a Whole and (a) Part}

An imbued paradox

Finally, the sub-centre cannot be conceived apart from the polycentric city at large; a system plagued by paradox and lauded for the numerous and broad structural changes (and opportunities) it engenders ${ }^{67}$. As the locus of this broader urban transformation - effectively, a polycentric microcosm - the subcentre may be understood to be equally-fated: simultaneously a hub, node and a place ${ }^{68}$; a structured object in a fluid field; largely autonomous yet fundamentally interconnected; a centre but not the centre; affirming an urban system that is both highly decentralized (widely seen to renounce the 'centre' as an idea), yet resisting it in favour of a more centralized logic (escaping the infinite wilderness of the city). The opportunities here are abundant: new social formations; a more individualised, yet more inter-connected society, new decision-making bodies, to name but a few ${ }^{69}$.

These paradoxes are not only crucial to the effective operation of the sub-centre in the wider region, but inherent to the idea of the sub-centre itself: indeed, differentiating it from both a 'suburb' and 'city-centre', and bearing implications that are not just functional, but plainly political, social and spatial. How the sub-centre deals with these two-fold, paradoxical forces, and the uncanny contradictions and opportunities they deliver is quite compelling. Certainly, these present an urban condition rife with potential. (fig. 15)

\footnotetext{
67 These have been alluded to earlier in this paper, but consist of centralization and decentralization, localized and globalized forces; dispersed and densified, for example. The broader structural changes and opportunities induced by a polycentric configuration are understood to be socio-economic and political, amongst others. See (Hall and Pain 2006) (Jaume 2011).

68 As pointed out by Worthington (2009)

69 There is much more to be said of these paradoxes and vast opportunities then can be offered here. See, for example (Lehmann 2010, 93-94) (Hall and Pain 2006)(Jaume 2011).
} 
Collectively, these ideas relay an understanding of the sub-centre that is complex, plural, and culturally-inflected, and one that inevitably transcends the economic and functional concerns stipulated by popular definitions.

Collectively, these ideas relay an understanding of the subcentre that is complex, plural, and culturally-inflected, and one that inevitably transcends the economic and functional concerns stipulated by popular definitions. Simply, this urban phenomenon cannot be understood merely on the basis of the densities or employment opportunities that initially motivate it; but rather is incumbent on an array of concerns that are, inter alia, political, social and cultural in nature.

With this expanded conception, the sub-centre may be seen as an emerging urban condition that is quite uncanny: conceptually distinct to both the city-centre and the suburb, and indeed, rife with uncharted opportunity (and potential). Still, dominant conceptions attest to a different reality, establishing priorities in the material constitution of the sub-centre in a way that struggles to impart these potentials. To this end, these ideas a condition of centrality; an embodied transition; a differentiated centrality and an imbued paradox - seldom characterise these rapidly-produced urban environments in practice: described more readily as themed, singular, efficient, functional and instant.

\subsection{Limited Processes of Forming the Sub-centre}

"But what are the relationships between the design of a city its form - and the life engendered by that form?"

-Frank Eckardt and Peter Kreisl, 2004

To return now to the second objective - to reconsider the making of the sub-centre that invariably sees the image prevail over the idea. Part of the current problem here clearly arises from the limited mediums, scales and strategies through which the sub-centre is afforded 'critical' attention.
Whereas larger scale gestures permeate strategies to develop and demarcate and generate the sub-centre (planning, infrastructure, transit-design, urban-planning, for example), smaller-scale mediums (spatial composition and built form, for example) are afforded far less attention in this regard. ${ }^{71}$ While these former gestures may uphold an economic and functional image of the sub-centre par excellence, their means to deliver or enact an expanded conception of the sub-centre are limited. To this end, the strategies of 'making' the sub-centre clearly need to be extended ${ }^{72}$

\subsection{The (un) Uncanny Abilities of Urban Planning}

Urban planning practice - readily deployed to bring these urban environments rapidly into being - invariably struggles to bring a broader understanding of the sub-centre to bear. With its penchant for amorphous, spatial conditions and generic, homogenized environments, its will to engender the sub-centre as a veritable centre, a nuanced space of paradox and transition, or a differentiated part of a larger system is limited. ${ }^{73}$ Less particularly, those aspects essential to the sub-centre as a public, cultural and social centre, yet often absent - user-attachment, sense of place - appear to lie beyond the capabilities of urban planning as a means of city-making ${ }^{74}$. Lehmann points out the obvious limitations of planning processes in constructing a nuanced, vital and authentic public culture within a sub-centre context, asking simply:

"how can [the] differentiation (required to achieve a public culture) be fully anticipated by the designer in the planning phases?"75

Indeed, while urban transformations at this scale easily reinforce an economic and functional understanding of these spaces - obliging the image of the sub-centre par excellence - they cannot realistically be expected to deliver an idea of the sub-centre as a vital, public or politically-differentiated central space, for example. Further still, as Peter Trummer

\footnotetext{
71 (Cladera, Duarte and Moix 2009) Lehmann 2010) (Dear 2011) (Gyoruvich 2011, 252) suggest that a broader range of spatial and formal pracices (including but not limited to architecure) must to be considered ices (inclucting but in relation to the emergent sub-centre and that policies of sub-centre 'generation' and demarcation should be extended to include these. 72 Though this assertion broaches general questions about urban generation, here I limit the discussion to the sub-centre, for obvious reasons.

73 Modern planning practice is widely apprehended for its inability to create quality public space, place identity, and user attachment, for example In these fastemerging urban spaces, where these planning principles may be applied holistically, this effect is often munning principles may be applied hollstically, this effect is often multiplied. (Gehl J. and Gamzoe L., 2001; Lynch K., 1960 and 1984; Gyurkovich J., 1999; Krier R., 2006; Krier L.,2011; Kantarek A., 2008; Wejchert K., 1974; Zuziak Z., 2008) in (Gyurkovich, 2011) 
suggests, modern planning practice may undermine such an understanding of the sub-centre ${ }^{76}$.

\subsection{A Limited Exception: disparity in plan and perspective, and the 'trumping' of built form}

Of course, the 'limited' ability of larger-scale gestures is not without exception: and one may be found in the case of Potsdamer Platz. Here, a policy of Kritische Rekonstruktion (critical reconstruction) gave rise to a Master-Plan cued from a traditional European City model: a means to relate to the politically and historically-significant site. (fig. 16) ${ }^{77}$ Through its familiar spatial and organisational composition, this masterplan gestures towards a notion of centrality and urbanity; and likewise, refers to the transition embodied in this new urban centre by acknowledging the former identity of the site in its urban morphology: delivering an 'appropriate' urbanity, as Lehmann argues ${ }^{78}$.

In this instance, and despite possibly being criticised for its nostalgic and restorative approach (rather than forwardlooking), a broader conception of the sub-centre as a space of embodied transition, veritable centrality, and culture of urbanity ${ }^{79}$, for example, is enabled through the planning. To this end, it may be argued that Potsdamer Platz attests to the sub-centre as an uncanny and distinct urban condition.

Yet, this strong gesture in plan is negated in perspective, insofar as large-scale commercialized architectural forms attest to an alternate reading. Notably, these built forms were subject to the aforementioned policy of 'critical reconstruction' - which prevented plot amalgamation; limited building heights; insured a diversity in architectural language; insisted on robustness through flexible building typologies and arcades at ground level to enable unrestricted pedestrian access; and established a material palette for the buildings (amongst others). These policies were premised on principles of creating a 'lively metropolitan ambiance' and social inclusion - and indeed, in terms of delivering this, the built forms are regarded to have been relatively successful ${ }^{80}$. Nonetheless, in their formal

\footnotetext{
76 Peter Trummer, for example, identifies modern planning's 'undermining' position towards understanding urban contexts as a unified idea (2013,52)

77 (Lehmann 2010,90)

78 Ibid.

79 The adoption of the European City model was a gesture towards the 'compact city' theory stipulated by Aldo Rossi, amongst others, to imbue a culture of 'density' in in this new urban centre. Lehmann (2010 90-92)

80 For example, a different designer was employed for each building to ensure'variety in architectural language and avoid homogeneity; and the material palette was established early on so that the buildings would create a "warm and inviting atmosphere". Lehmann (2010, 90-92).
}
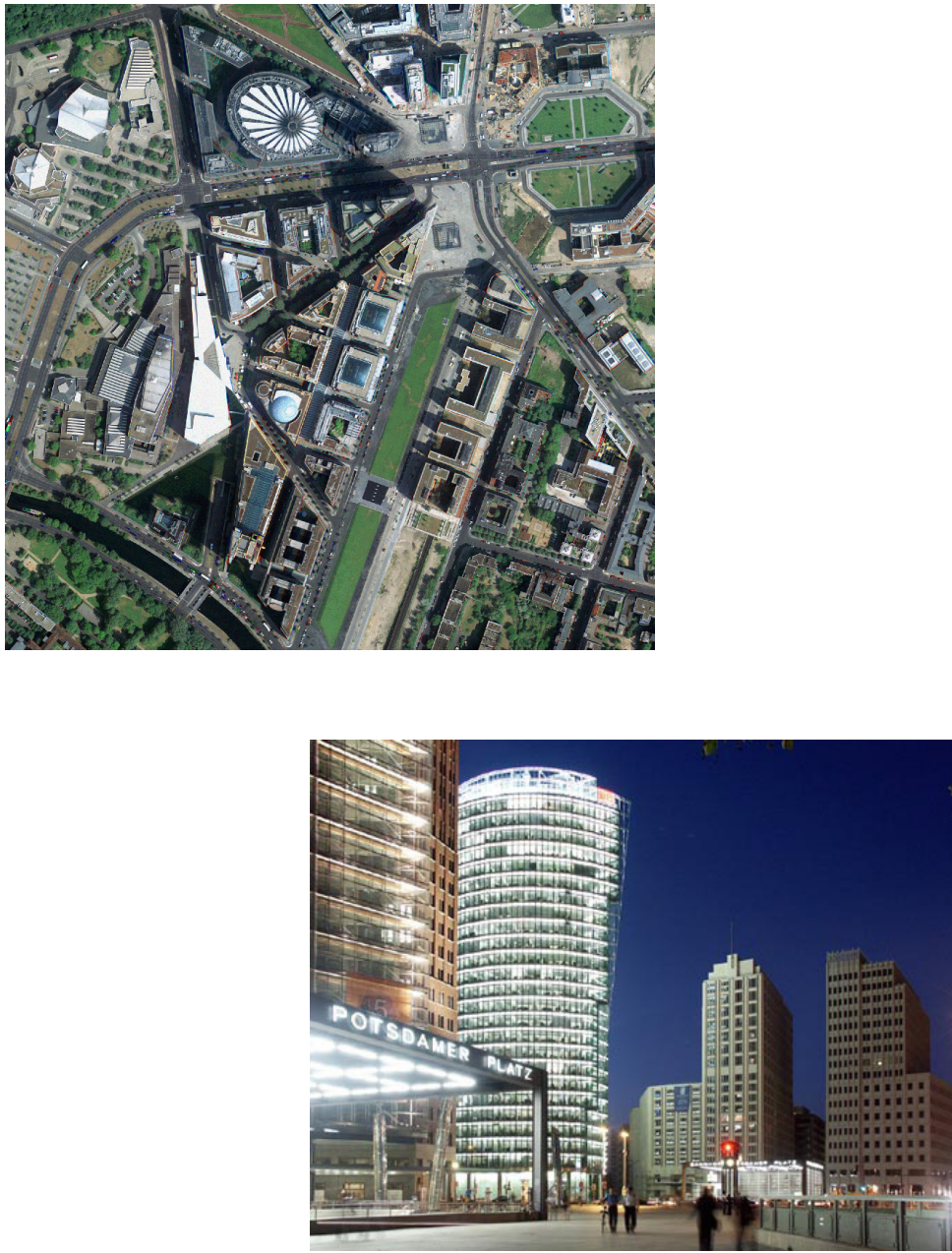

Figure 16. Potsdamer Platz's Master Plan by Renzo Piano Studio included a compac block, mixed-use typology a piazza, and a street-scape - adopting a traditional means to relate to the historic site. European City Centre, as a

\begin{abstract}
Figure 17: Disparity in plan and perspective. The largescale, modern, built forms have summoned reviews of the context as a 'shopping mall city'. Here the commercial, large scale and modern language of the built form effectively negate an effort towards a more traditional centritiona y that ees Potsdamer Platz describe as an inauthentic urban place, despite its commercial success.
\end{abstract}

language - readily perceived as commercial and commodified - the built forms betray an urban space compelled by commercial and economic imperatives: indeed, far from the contextually-appropriate urbanity or embodied transition subscribed to in plan. (fig. 17)

To this end - and, somewhat ironically - Potsdamer Platz has summoned criticism for its lack of cultural and contextual authenticity $^{81}$ : indeed, widely noted for conforming to North American trends ${ }^{82}$. In short, the notion of a shopping mall city or Berlin's answer to Las Vegas dominates readings of this urban context over any culturally-sensitive or appropriate notion of a centre. $^{83}$
For example, being called the 'Americanization' of Berlin. 
Figure 18: The potency of architectural passivity. The dominance of economic and functional imperatives are encapsulated by the 'architecture' of the shopping mall. Often considered the heart of peripheral urban centres, these often present a monotonous asthetc and over-scaled unmodulated form and to their urban contexts - indeed, struggling to afford it a comparative role to the city-centre, as a place of political/cultural/social gravity. This neglect to actively engage a broader idea of these urban contexts, through built form, has resounding effects for the way these urban settings are perceived. Shown above is the Lower Hutt Shopping Mall in Wellington, New Zealand: a commercial centre whose material and formal languag does little to imbue its urban context with a quality public culture - but rather attests to an abrupt fabrication of urbanity.

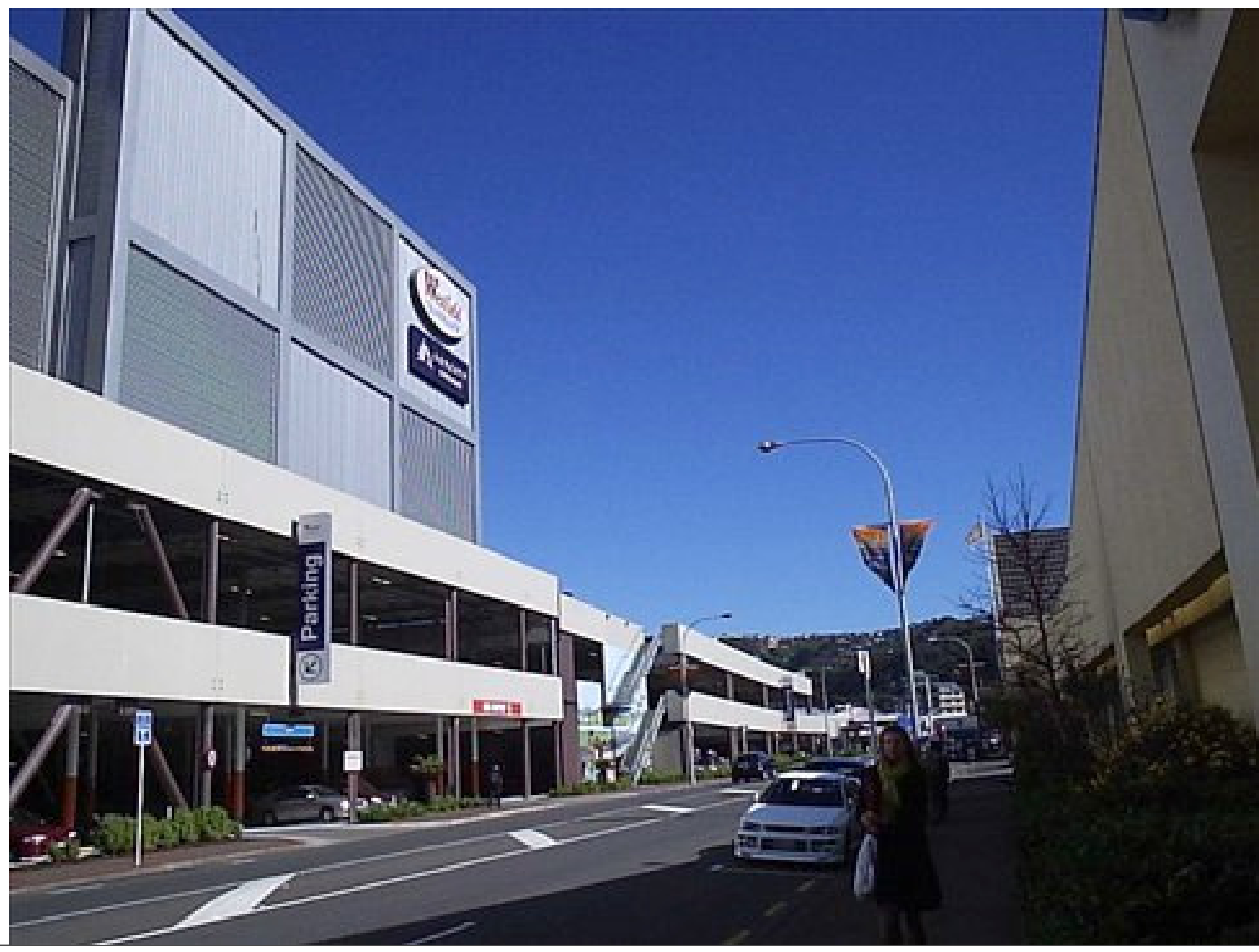

\subsection{3 in the Sub-centre, of the Sub-centre}

The implications here are quite straightforward. The 'trumping' of built form in the case of Potsdamer Platz belies, more broadly, the inevitable role of architecture in imbuing an understanding of these new urban spaces: somewhat irrespective of what happens at a larger scale (and indeed, despite the better intentions of master-planning, for example). Peter Trummer attests to this observation in a more general sense, suggesting that:

"...we do not relate to a territory anymore, we only relate to the buildings - the architecture that is the figure and ground of the city" 84

Here, the notion of the Potsdamer Platz as a shopping-mall city offers a final point of contemplation. If a paradigmatic'architecture ${ }^{85}$ of the sub-centre does exist, surely its logical manifestation must be in the shopping mall: a building type as equally associated with peripheral urban centres (or, indeed, urbanizing suburbs) as their inability to compete with city-centres on a cultural or social basis. ${ }^{86}$

Indeed, if the shopping mall as an urban form can be seen to attest to any' image' of its wider urban context, it is a functionally or economically oriented one par excellence. Appropriating a Koolhaasian measure of 'productive' urban architecture here, the shopping mall may be described as "a characterization of the economic determinants... [of] construction within the city" but fails to "offer directions for aesthetic analysis and cultural interpretation" ${ }^{\prime 87}$. Broadly speaking, on account of its often

\footnotetext{
$84(2013,57)$

85 Or rather 'built form'

86 In Christchurch this is definite reality - where shopping malls charac-

terise these peripheral urban-centres spaces par excellence.

87 As cited in in (Martin 2001, 197)
}

bare or crude aesthetic, unmodulated tectonic expression, vast-scale, modern materials and minimal formal articulation (to name but a few) (fig. 18) the shopping-mall typically does remarkably well to reinforce an understanding of the urban sub-centre as:

"a reflection of the experience economy [...] with [its] focus on efficiency, functionality and convenience ${ }^{\prime \prime 8}$.

\subsection{The Potency of Architectural Passivity}

Inasmuch as these de-facto material and formal tendencies may be seen to reinforce an idea of these peripheral urban settings as plagued by economic and functional imperatives, they can equally be seen to neglect a broader idea thereof: as a vital socio-cultural centre; a differentiated place within a larger urban structure (beyond function) ${ }^{89}$; or indeed as a holistic alternative to the city-centre. Rather than to any notion of embodied transition, these tendencies attest to a blunt fabrication of urbanity.

While the tendency to reflect these dominant economic and functional imperatives is not entirely fortuitous (and is much more complex than I have afforded in this discussion) ${ }^{90}$, the passivity in regards to a broader idea of the sub-centre is undeniably potent, nevertheless. Moreover, though the shopping mall may represent the apotheosis of architectural passivity in regards to a sub-centre context - indeed, as the

89 Though they may be differentiated functionally, they are less often culturally so.

90 That is, there are many more complications than I have afforded here in regards 'shopping malls' as an urban type; and of course my explanation is necessarily general. 
'heart' of these peripheral urban settings in many cases - it belies a wider disciplinary tendency to perpetuate a narrow idea of this urban space: simply, by neglecting to actively engage a broader one ${ }^{91}$.

\subsection{Pointing out the Obvious: the sub-centre as a desperately architectural phenomenon}

\section{Architecture as (the) medium of the sub-centre}

"The paradox of the city is that it intrinsically demands design, yet inherently resists it"

Dann Cuff and Roger Sherman, $2011^{92}$

While the varied examples above may often depict comparable formal or spatial results ${ }^{93}$, ultimately the criticism here is directed at the limited critical processes (and practices) currently afforded to the sub-centre, rather than to the built outputs themselves. Realistically, there is only so much planning, transit-oriented gestures, and infrastructure can do (or have will to do) in constructing a more holistic understanding of the emergent sub-centre: to establish them as social, cultural and political centre, or to impart them with an urbanity that can may see them operate as a veritable and differentiated alterative to the city-centre ${ }^{94}$. Certainly, these methods of urban generation struggle to attest to a more nuanced idea of the sub-centre as space of paradox and transition.

Comparatively, architecture's relationship to the sub-centre as an urban idea is quite potent, instrumental and indeed, somewhat constitutive ${ }^{95}$. Yet - in as much as it may be considered a capable - and even culpable-medium - its role in regard to this broader idea of the sub-centre appears to be somewhat passive: or rather, affirming and helping to perpetuate a version (image) of the sub-centre that is discerningly narrow ${ }^{96}$. Indeed, to this end, it seems the significance of the sub-centre as an urban

91 As seen in previous sub-centre examples discussed throughout this paper so far.

paper so f

93 The shopping mall 'aesthetic', modern planning for example.

94 Though, in some ways, this operates as a general critique of the limitations of these methods of making city: applied to the sub-centre en masse, and a rapid rate, the effects appear to be multiplied.

95 That is, insofar as it plainly contributes to a 'deeper' understanding of these urban spaces, and in turn, affects how they materialize and operate.

96 As seen in the examples above, architecture (built form) often attests diligently to the commercial and economic imperatives of these contexts par excellence (exemplified by the shopping mall). As argued, this is not fortuitous, but is incredibly potent, no less. idea appears to have been largely neglected in architectura practice. As Eve Blau, a professor in urban form at the Harvard Graduate School of Design remarks:

"The significance of [recent urban changes] for architectural practice - and in particular, for the ways in which architects operate within these contexts and networks - is still quite uncertain" 97

Taken seriously, the emergent sub-centre as an urban condition is equally problematic and opportunity-ridden. The implications here are quite transparent: firstly, that architecture is a potent sub-centre medium, inevitably instrumental on the way this urban space is conceived; secondly, that to avoid a deplorable fate for the emergent sub-centre, architecture must be consciously considered and addressed such. Put otherwise, tactics towards 'making' or generating the sub-centre must be extended to make room for architecture.

Of course, the call for architecture to enter into an urban debate is by no means uncommon. Its unique role in allowing "images concepts, fantastics, politics and planning theories" to be brought to bear in urban development, for example, has often been expressed - where it is seen to offer something in scale, material and form that other urban mediums cannot. ${ }^{98}$ Likewise, the need for architecture to re-think its engagement with new 'altered' urbanisms is variously recognised - also, citing architecture's often-unharnessed potential as a potent urban medium ${ }^{99}$. Yet, in an emerging sub-centre context arriving rapidly and en masse - these convictions are not merely pertinent: the inclusion of architecture within such debates is, in fact, utterly imperative.

In this way, rather than lying beyond architecture's grasp or concern - it may be seen that the emergent sub-centre is indeed a desperately architectural phenomenon.

Of course, the call for architecture to enter into an urban debate is by no means uncommon [...] Yet, in an emerging sub-centre context -arriving rapidly and en masse [...] the inclusion of architecture within such debates is, in fact, utterly imperative.

\footnotetext{
97 (Blau 2010, 4)

98 The call to reposition architecture's 'productive' role in urban generation, and to enable a more transdiciplinary debate is common. See, for example, Eckardt and Kreisl $(2004,14)$ 99 See for example (Dear 2011, 226-30)
} 
Such an agenda for architecture need not signal a resort to historic or nostalgic ideas, utopian visions, iconic gestures or crude efforts to symbolise or represent the sub-centre, however.

\subsection{Agenda, by way of Conclusion - The Sub-centre as Architecture's Project}

"What [does] rereading the city as an object mean to the discipline of architecture and to the form of the city itself ... what is the role of the architectural object as a part that makes up the city........ [through what modes can architecture] give us a deeper understanding of the [city]?"

- Peter Trummer, $2013^{100}$

"What is needed is an idea, a trick, a concept...that exacts more from architecture than mere usefulness"

- Hans Frei, $2009^{101}$

"[This] altered urbanism necessarily requires innovation in architecture"

- Michael Dear, $2011^{102}$

Finally, we may flip this assertion on its head. Inasmuch as architecture may be considered a fertile and necessary medium in making the sub-centre - an urban context and idea by now inseparable - its relationship to this urban condition must be critically explored: its instrumental and somewhat constitutive role in generating this urban space actively (or retroactively) addressed. Insodoing, architecture might consider its role in the sub-centre as both catalytic and productive: or, equally, as a medium and constituent thereof.

Such an agenda for architecture need not signal a resort to historic or nostalgic ideas, utopian visions, iconic gestures or crude efforts to symbolise or represent the sub-centre, however. Rather, it suggests that while delivering urban densities and programmes to these emergent spaces, architecture may also consider itself in relation to the sub-centre at large: engaging it according to the broader ideas laid out above, in an effort to impart a deeper understanding of this urban space, in this urban space ${ }^{103}$. Here, architecture should not only endeavour to position itself in or relate to the emergent sub-centre but position itself in relation to it.

Ultimately, this agenda brings architecture's mode of operativity, as urban form, into fundamental question. How might it deliver ideas of centrality and urbanity that are social and cultural rather than just economic? Through what means can it impart a deepening understand of the sub-centre, in the sub-centre? How does architecture's role in this urban context - that is at once centre and periphery, yet neither - differ from in the suburb or the city? How can it actively relate to and engage the sub-centre as a public environment and space imbued with transition? How might it catalyse an understanding of the sub-centre as part of a larger system, and respond to it as polycentric-microcosm imbued with paradox and opportunity? How does the sub-centre (as a centre but not the centre) inform architecture's role? Indeed: what does the sub-centre mean for architecture - and vice versa - what can architecture do in, to and for this emergent context, that so desperately requires it?

Certainly, these questions are not easily-broached, and are even less easily-answered: rather, they invite endless debate. Still, if there is a chance of preventing these new urban spaces from the deplorable fate of being holistically characterised by the economic and functional imperatives bringing them into play - and likewise, if they are possibly to emerge as public, cultural, genuine urban places, differentiated and integrated in a polycentric system - these questions are vital, nonetheless.

For architecture then, the emergent sub-centre is clearly no peripheral issue. It is absolutely central.

What does the sub-centre mean for architecture - and vice versa what can architecture do in, to and for this emergent context, that so desperately requires it? 


\section{"... is the aesthetic \\ of the individual \\ - building radically \\ to be disjoined}

from the problem

of the urban in
such a way that the

problems raised

by each belong and

remain in separate

compartmeints [?]

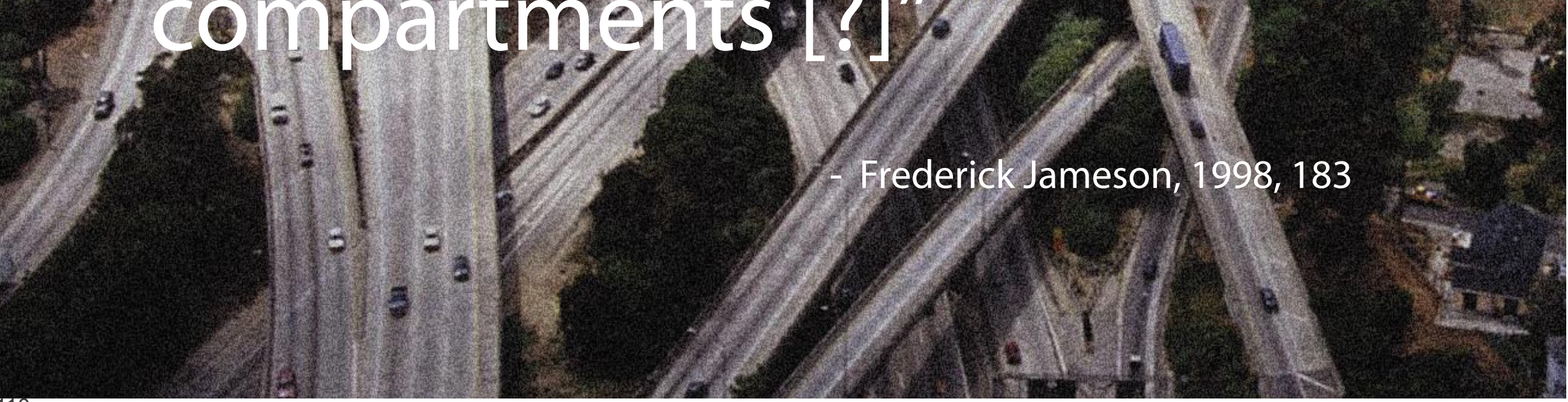




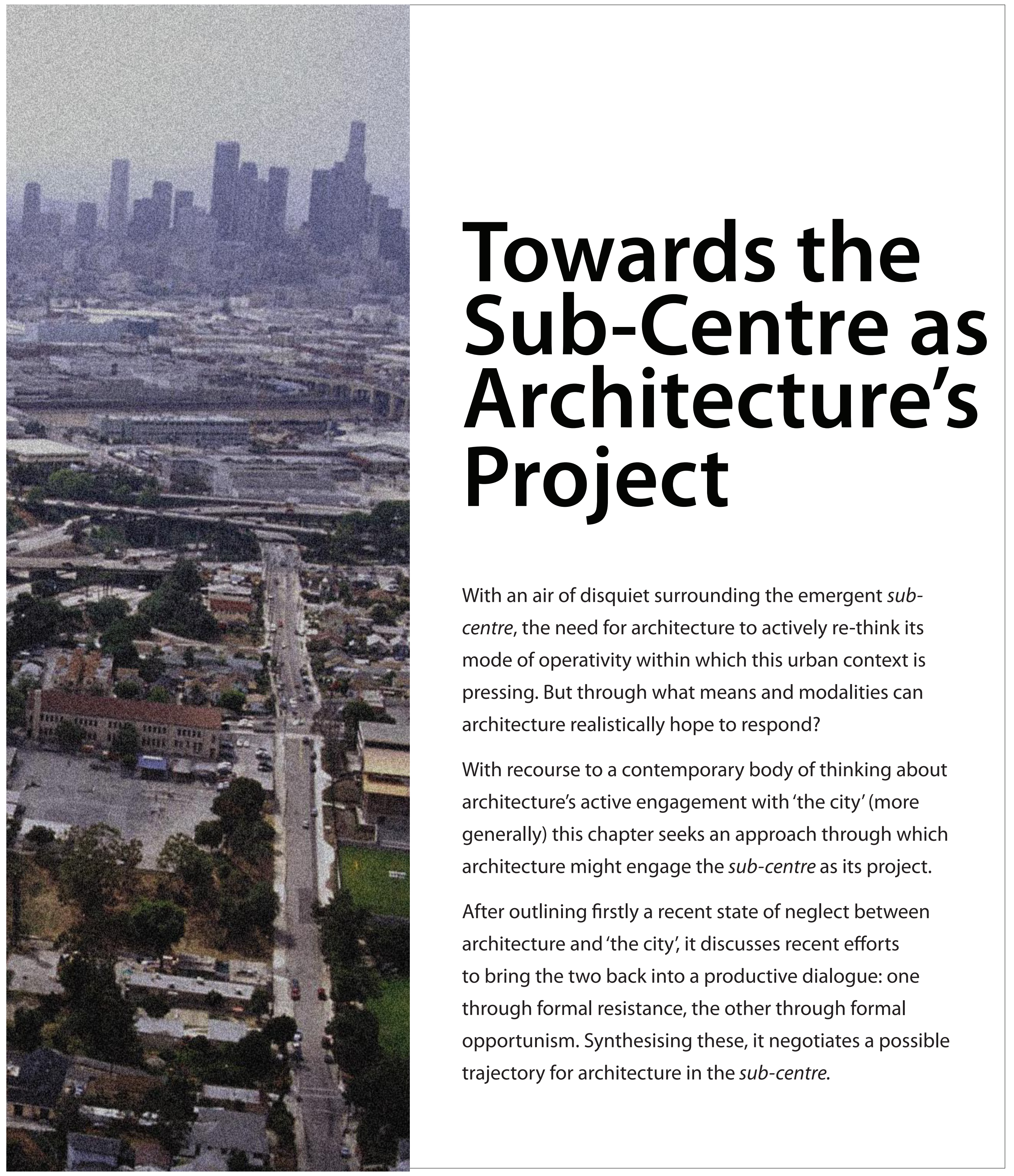




\subsection{Architecture and The City: a brief history of a twisted love-affair}

"I don't think architecture could do without the city.. It's very rarely a single object that exists on its own; instead, it should always try to accelerate or transform certain urban phenomena. That's how I look at it."

Bernard Tschumi, 2012, 131

Through the course of the $20^{\text {th }}$ century, the conviction that architecture could relate, productively', to the idea of the city, spawned a string of diverse architectural explorations ${ }^{2}$. Broadly speaking, these divulged architectures' ability to describe, reveal, evoke, constitute, or enact the city as a political and socio-cultural construct - and to act upon or advance it, in so doing $^{3}$. Today, these intermittent tendencies have amounted to the perception of a tumultuous, problematic but nevertheless passionate love-affair between architecture and the city ${ }^{4}$, of which the terms of engagement were frequently re-negotiated through formal and informal homologies, visual and material tactics, and a host of avant-garde efforts, during the latter decades of the $20^{\text {th }}$ century.

\section{Upon the city, architectural form}

A number of significant $20^{\text {th }}$ century projects can be seen to have attested to this way of thinking par excellence. Rem Koolhaas's famed Delirious New York - A Retroactive Manifesto for Manhattan, for example, divulged architecture's relationship to a city beset by urbanising forces: describing a "patheticallyone-sided, unrequited love" where the city inspired, but never responded to, an overly-doting architecture (assuming the role of the rejected lover) ${ }^{5}$. In a European context, Aldo Rossi explicated the architectural object as an 'urban artefact' and

\footnotetext{
1 The term productive here suggests generative and active. 2 The 'idea of the city' as used here refers to a 'collective meaning of the city'that is historically constituted and concerns itself with the "civic and symbolic function of human settlements and coexistence... and. [thel distinctive character coupled with the need to accommodate difference" (C. C. Lee 2011), and is used by contemporary thinkers to der. describe the city as an political and cultural endeavour. The notion that the individual building (as urban artefact) could to relate 'productively' to the idea of the city' - that is, act upon it and even reconfigure it- arose from a critique of modern urbanisation's effects on the city, and the desire to reinstate pre-existing urban values. For more see Aureli (2011) Eisenschmidt (2012), (Rossi 1982) (Lee and Jacoby 2011).

3 The endeavours-of-interest here were less concerned with the individual building as a physical 'landmark' giving legibility to the city as a whole (as famously discussed in Kevin Lynch's The Image of the City (1960); but rather, in the architectural object's ability to embody, communicate, and evoke the city as a concept or idea - to impart and understanding thereof, and to advance it as an on-going cultural and political project.

4 Rem Koolhaas initially described the relationship in this fashion in 1997. but today, the metaphor is widely adopted (Somol 2012,108). 5 Koolhaas influentially discussed architecture's engagement with an idea of the city based on the 'culture of congestion' (1994).
}

'index' for the city: hypothesising that through their distinct morphological and typological character, individual buildings may embody, progress and construct a collective 'image of the city' (positing a pars pro toto relationship, effectively) ${ }^{6}$. On a different tangent still, groups such as Archigram and Superstudio sought to radicalise the relationship between Architecture and The City, with vast mega-structures that formalised infinite expansion of the $20^{\text {th }}$ century city beset by urbanisation: in so doing, providing active, architectural commentary on the city as an idea (see figures 20-25).

Notwithstanding their vast differences, these projects betrayed a common infatuation: the possibility of architecture's relationship to and active engagement with the city as a sociocultural and political phenomenon. In each, architectural form (as urban form) was harnessed as a means to understand explore, and deliver bigger questions about the city, and in turn, architecture's potential instrumentality upon it. To this end, underlying such projects was a bilateral conviction: that architecture had some offering to make to the city $^{7}$, and likewise, that understanding(s) or idea(s) of the city played a vital, influential and necessary role in architecture's conception. On both counts, architecture and the city could be conceived as complementary forms, inextricably bound to one another by what Robert Somol has described as an internal and relational dialectic $^{8}$. Here, in as much as the relationship between architecture and the city was considered of fundamental importance, the city was understood as architecture's utmost project: its context, objective, impetus, provocation, motivation, and raison d'etre

Notably, while on the one hand this type of thinking about architecture's exchange with the city is often associated with architecture's more discursive and speculative undertakings on the other, inasmuch as it manifested often as design strategies and formal operations, the conviction was able to inflect architectural design practice (unbuilt and built), to some degree ${ }^{10}$. In essence, in as much as the city could be considered architecture's project, it was held that architecture should - and moreover, could - actively address the city as such. In this way, the dialogue between architectural form and city was of crucial concern.

6 Rossi's "The Architecture of the City" discussed architecture's role in the construction a 'collective' image of the city, focusing on architectural language, composition, form and typology (and, effectively, a dismissing the urban potency of function):essentially arguing that urban architecture, must reflect the spirit and personality of the city. (Rossi 1982)

7 Not in the sense that it was 'prophetic' or deterministic, but rather, 7 Not in the sense that it was 'prophetic' or deterministic, but rather,
that it could to some degree influence or change the way we think about or understand the city. See, for example (Cupers and Doucet 2009). 8 Somol lists figure/ground; part/whole; object/field, public/private, for example, as basic dualities underlying architecture-city's dialectical relationship or complementary balance $(2012,110)$

9 (Eisenschmidt, 2012) describes the term 'project' in this way. 9 ' notion of typology as a strategic architectural device within the city, for example. See (Rossi 1982) More broadly, Robert Somol suggests that architecture's formal operations were largely 'conflated' with those of the city. (2012). 

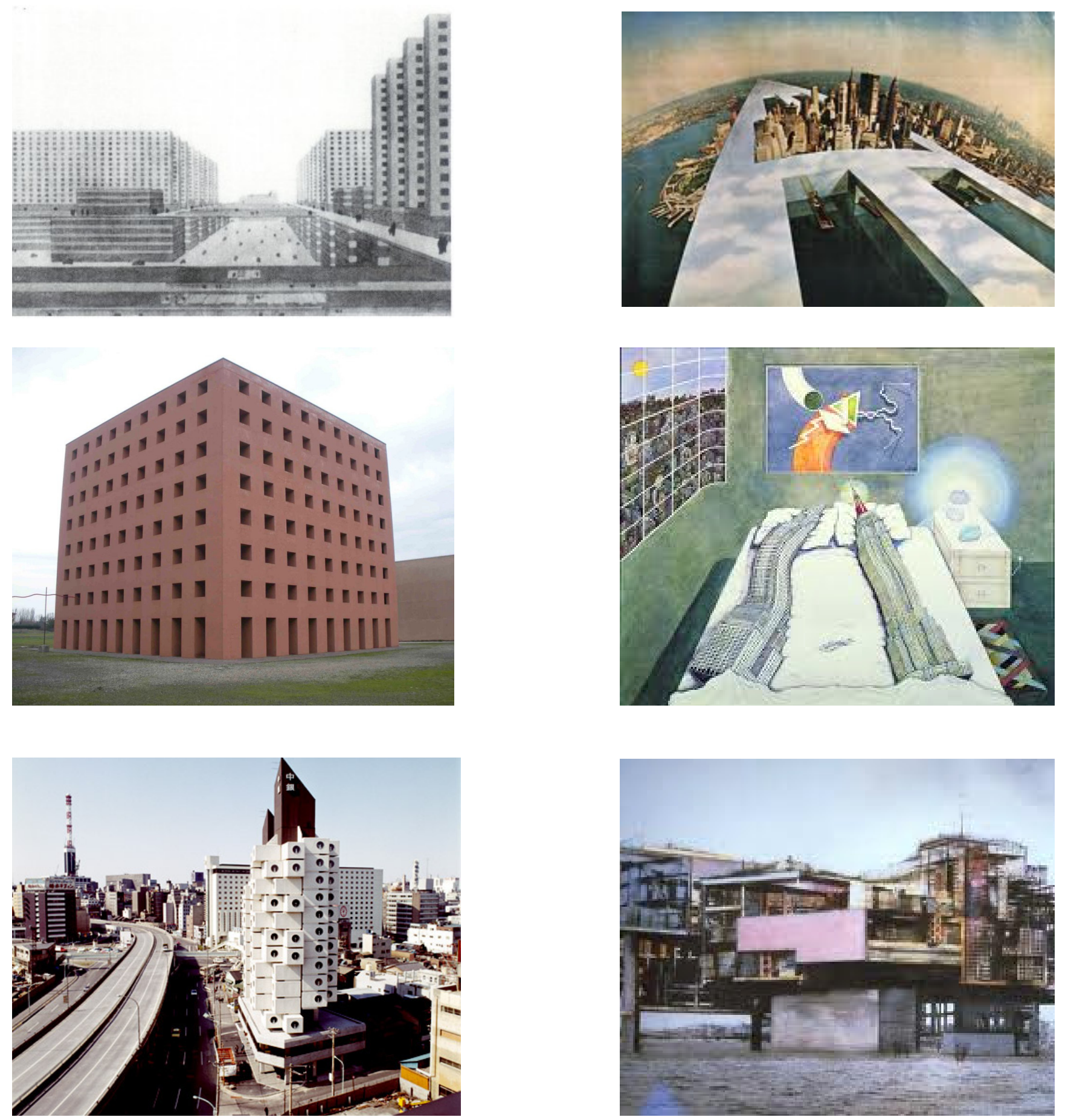

Notwithstanding their vast differences, these projects betrayed a common infatuation: the possibility of architecture's relationship to and active engagement with the city as a sociocultural and political phenomenon.
Figure 20-25: The City as Architecture's Project. Explorations of architecture's relationship to the city can be seen in number of $20^{\text {th }}$ century architectural projects. From top left, clockwise, Ludwig Hilberseimer 1920s metropolis',

Superstudio's radical megastructures; Aldo Rossi 'urban artefacts' grounded largely in typology; Rem Koolhaas's doting architecture of the metropolis, Constant's New Babylon (1959-74); and Kurokawa Kisho's Japanese Metabolism (1972). These projects may be seen to have regarded the city as their project, each offering ideas about the city-at-large - in its transforming state for example - through their formal, compositional and tectonic strategies.

For more on these projects, see (Hilberseimer 2013); (Rossi 1982); (Wigley 1998); (Koolhaas 1997). 


\subsection{No Longer Pals: a recent state of neglect}

"Surprisingly enough, relatively little work and research is being done today on the relationship between architecture and the city. There seems to be a split between the two; the architect all too often designs singular objects, without taking urban circumstances into account. This is an unfortunate situation because it's an opportunity that is missed."

- Bernard Tschumi, 2012, 131

"[No statistics are required] to note that the building as a singularly authored object is responsible for a relative trickle of the world's spaces, while a fire hose blasts out the rest."

- $\quad$ Keller Easterling, 2012, 58

In recent decades however, architecture and the city have become increasingly ambivalent terms, and the possibility of a productive relationship between them has largely been dismissed as a vacuous promise ${ }^{11}$. Reflecting the common perception that architecture has lost', or been 'beaten' by the city, the turbulent-yet-passionate romance described earlier is today more readily described as acrimonious, ambivalent or indifferent ${ }^{12}$. To paraphrase Professor Ron Witte of Rice University: architecture and the city are "no longer pals"13

\section{Urbanisation's Triumph: the city no longer exists}

In the first instance, this division is described as a post-mortem response to urbanisation's triumph over the city - amounting to the perception that'the city no longer exists! ${ }^{14}$ - and according to which notions of architecture-city have been widely supplanted by those of architecture-urbanisation.

\footnotetext{
11 This division is variously attested to. See (Eisenschmidt, 2012) (Tschumi, Importing the City into Architecture: An Interview with Bernard Schumi, 2012) (Witte 2012,75).

2 See for example (Witte 2012, 77) (Castle 2012, 5).

13 It is widely argued that architecture has not been able to keep up with huge shifts to the geopolitical urban landscape, in recent decades, for example, meaning architecture and the city have becom disengaged. See (Somol 2012) (Eisenschmidt, 2012) (Tschumi, Importing the City into Architecture: An Interview with Bernard Schumi, 2012 Witte 2012).

14 (Eisenschmidt, 2012, p. 14)
}

Expanding on this idea, Professor Michael. J Ostwald from the University of Newcastle suggests that urbanisation's rampant and pervasive presence has fundamentally reconfigured architecture's role in an urban context ${ }^{15}$. He asserts that architecture has become increasingly submissive to urbanisation's capitalist, economic, integrationist, a-political and managerial dictates in recent years: relating to urbanisation on its own terms, rather than to the political ideologies and systems forming architecture's point of reference to the city in the past ${ }^{16}$.

This assertion belies a familiar view: that the potency of the city both as a political construct and a formal, definable entity has been diminished by urbanisation's homogenizing, a-political and amorphous forces - if not entirely dispelled ${ }^{17}$. To this end, insofar as the city no longer exists in the political, unified capacity it once did, architecture need not engage it as such but rather, may direct its attention to urbanisation's complex and ever-demanding processes ${ }^{18}$.

Yet, with the post-political 'urban haze'19 as its reformed backdrop - one characterised not only by economic and managerial systems, but equally a smooth, spatially-endless and unstructured nature - architecture is seen to operate with a reduced potency: its homologous relationship to the city through form inevitably belittled by urbanisation's penchant for enormity, growth and formlessness ${ }^{20}$ (figure 26). Simply put: on account of both the city's diminished existence as a conceivable entity, and architecture's reduced capacity to relate to it in a part-to-whole fashion, the two may be seen to have entered into a state of mutual ambivalence.

\section{... insofar as the city no longer exists in the political, unified capacity it once did, architecture need not engage it as such - but rather, may direct its attention to urbanisation's complex and ever-demanding processes}


Figure 26: Urbanisations penchant for growth, expansion and 'formlessness' (and the city's increasingly amorphous nature) is often seen to be contest with architectural form, effectively (s) relinquishing architecture's productive relationship to the city.

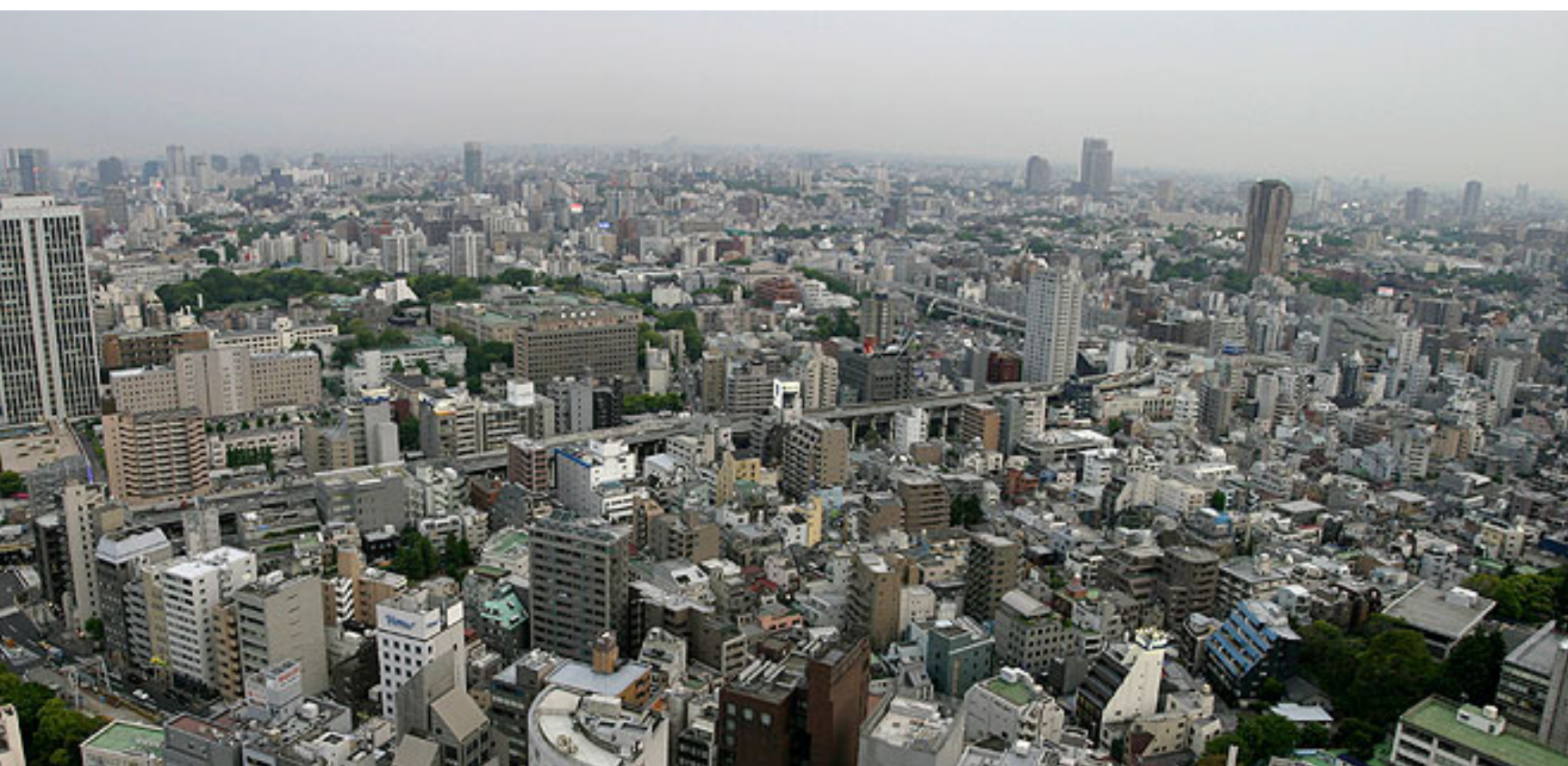

\section{From Constitutive to Bare Constituent:} architecture as fluff around the edges

Formulated slightly differently, those same urban imperatives understood to have relinquished the city as a formal, political idea have also fundamentally adjusted architecture's role in the city - as an urban constituent ${ }^{21}$. Where contemporary urban paradigms such as 'housing urbanism', 'landscape urbanism', 'transport-orientated urban development' inherently suggest that the progression of the $21^{\text {st }}$ century city is enabled through infrastructure, new modes of density, and transit, architecture's role in giving face to or constituting the city has been diluted ${ }^{22}$. To paraphrase Brendan Cormier, editor of architectural publication Volume: the city is no longer reinforced architecturally, but is understood and defined through alternative and often more intangible means. ${ }^{23}$ In short, though architecture is in the city (and at an unprecedented rate, in fact) it is no longer of the city.

To this end, insofar as the city (in whatever capacity it exists) no longer wholly requires architecture, it has effectively rejected architecture as necessary and repositioned its role as such $^{24}$. Accordingly, architecture is more readily construed as a facilitator of urban activity (performing urban functions, be it density or transport) than an index or agent for the city - and in this sense, is regarded more often as reactive than productive. ${ }^{25}$ Affirming this ever-marginalised and non-constitutive role, Ron Witte remarks that architecture has become mere "fluff around the edges." 26

\footnotetext{
21 That is, as something that makes up the city - not only physically, but conceptually and politically.

22 For more on these concepts see (Lyster and De Jong 2011)

(Waldheim 2006): (Curtis, Renne and Bertolini 2009) respectively.

23 Historically, architecture has re-enforced the symbolism of the city, as a centre, by 'representing what aspect of society was most dominant' - a church, for example. Today, this is seldom the case, and it is more often understood through information or data, for example. (Cormier 2012, 4)

24 As Helen Castle has argued $(2012,5)$

25 (Eisenschmidt, Stranger Than Fiction: A Mission Statement 2012, 15) 26 (Witte 2012, 77)
}
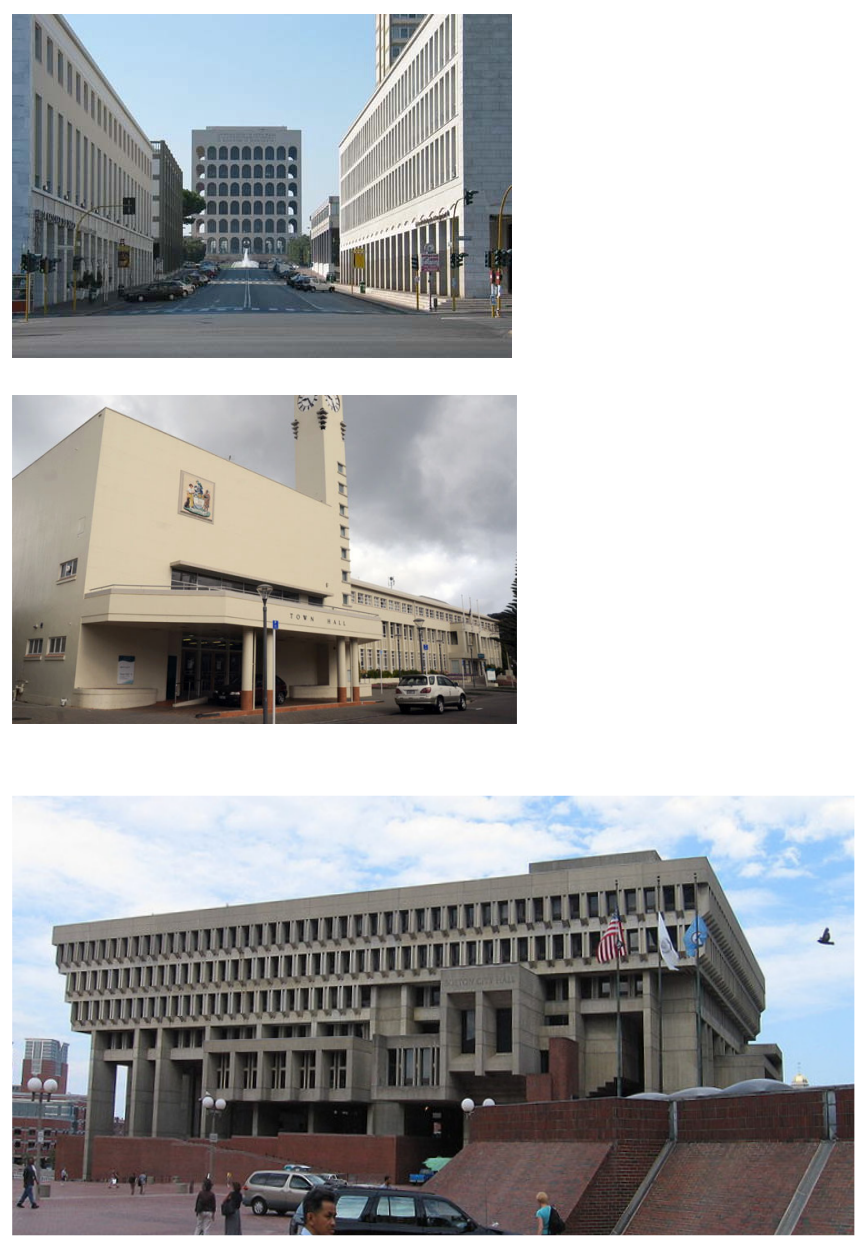

Figures 27-29: the city reinforced architecturally. Whereas in the past the idea of the city has indeed been understood and reinforced architecturally, today, the city is readily understood to be progressed through housing, infrastructure or transit: meaning architecture's role has increasingly become one of facilitation increasingly become one of facilitic
rather than constitution. Above, reinforcing the city as a place of political ideology: from the EUR in Rome; to the Lower Hutt Town Hall, to Boston City Hall. examples of architecture relating to and 


\subsection{Architecture in Accordance: the impertinence of being critical (upon the city, no less!)}

"Architecture has, to a large extent, abandoned the city"

- Dana Cuff and Roger Sherman, $2012^{27}$

Certainly, neither urbanisation's diluting effect on the city nor its coincident re-configuring of architecture's role therein appear to have left much room for a part-to-whole discussion about architecture and the city. Yet, if the dialectic of architecture and the city has been rendered futile by urbanisation, their disparity has only been further consolidated by recent architectural practice $^{28}$.

Inasmuch as the recently-dominant laissez-faire ethos broadly encompassed by the post-critical impasse - ascribes architecture no political potentiality, no agency and no ability to adopt a 'critical' standpoint, it has afforded architecture little reason to engage the city as its project ${ }^{29}$. Rather, this highlypragmatic and realist disciplinary position has effectively dismissed any active, productive or critical engagement between architecture and the city as idealistic, irrelevant, and foremost, beyond architecture's scope ${ }^{30}$. Governed instead by a set of revised priorities which do not understand the city as a conceivable idea (or object) but rather 'accept' it as a limitless source of information through which to sort - this dominant disciplinary stance has confirmed architecture's urban role as one of obligation and facilitation, rather than production (and, even less, constitution)

To this end - and especially compounded with the persisting attitude left over from the late $20^{\text {th }}$ century-hubris that "architecture should do building [...] urbanists should do cities" 31 , the post-critical moment hardly bodes well for architecture and the city to keep up their already precarious rapport. To paraphrase Professor Peter Trummer of Innsbruck University: far from engaging the city as its project, recent architectural practice has served to undermine $\mathrm{it}^{32}$.

28 See for example (Eisenschmidt 2012, 14) (Witte 2012, 58) (Somol 2012)

29 (Macarthur and Stead 2012) argue that within the post-critical moment, there is a broad consensus that architecture in a capitalist environment has no political potentiality, nor critical agenda (135). 30 While this post-critical moment is not directly formulated on architecture's relationship to the city, its underlying 'non-critical' and pramatic agenda easip suggests that a critical'positoning to the city at large is likely outside architecture's main objective.

32 (Trummer 2013, 51)

\section{Curatorial Strategies (to deny the city, positively)}

Broadly speaking ${ }^{33}$, this popular disciplinary outlook has summoned a body of 'curatorial' design strategies that decisively affirm architecture's facilitating agenda in the city (and in effect, its passivity in regards to the city as a project $)^{34}$. Here, data-driven, mapping, process-based, coding and parametric techniques prevail: each looking to processes and paradigms extraneous to the architectural discipline itself, in order to allow architecture to 'respond' to the multifarious and complex challenges imparted to it by urbanisation. In this way, and as Professors Grahame Shane and Brian McGrath of Cooper Union and Parsons School of Design have identified, the fluctuating understandings of the city seen above have not only influenced architecture's perceived role, but fundamentally, its modes of practice. $^{35}$

\section{In-Form : a 'resultant' mode of disciplinarity}

These curatorial approaches have been invariably problematic for architectural form's active, intentional and conscious engagement with the city. Insofar as architecture's visual and formal predilections are held as inept to deal with the multilayered complexities of urbanisation on their own, they have effectively become resultant: derived from something external and without their own 'agenda' per se. With this diminished disciplinary intentionality, urban processes have often dictated or even 'kidnapped' architectural form - in a way that Ron Witte suggests has amounted to pseudo-functionalism, and indeed "something as close to unassailability as an architect could get" $^{36}$. (FIGS) To this end, architecture's formal consciousness, integral within previous efforts to engage the city, has often been flung aside as superficial or redundant ${ }^{37}$. Certainly, it has seldom been predicated on engaging the city as a project.

\section{A Normative, Built State of Neglect}

Whether on account of its curatorial, a-critical, or 'a-formal' 38 predilections, recent architectural practice has amassed a body of urban architecture described variously as passive informalisms, formless, iconic, kidnapped form and seen to

\footnotetext{
33 The 'post-critical' does not present a position or 'stance' so much as a reaction to taking such a position (and attempts not to do so itself). Nevertheless, it has become associated with certain modes of practice. 34 Operating 'within' the conditions is a fundamental tenet of postcriticality; as opposed to 'criticality's' premise of maintaining some 'distance' from of the conditions it examines (Toorn 2005). $35(2012,641)$

36 As Witte suggests, the focus on informational, programmes and urban processes have "[become] the go-to means of assuring that architecture was not pandering to the vicissitudes of formal doodling" $(2012,77)$

37 Neil Leach has noted that conversations around architecture's visual instrumentality for example have been deemed 'superficial' and subjective in recent years (2003) Likewise, Ron Witte suggests that 'form' has becoming a'dirty word' in recent practice $(2012,77)$. $38 \mathrm{I}$ use this to refer to a relinquished formal consciousness, or to forms
} are that are'resultant' or passive - dictated by something external. 
be "mimicking new urban formations" 39 . In as much as these tendencies are seen to oblige urbanisation's formless logic - as Robert Somol writes "following the city down the rabbit hole of formlessness" 40 - and in so doing, affirming its economic, capitalist and a-political imperatives tout court; they are equally seen to negate the city as a political, formally-oriented and stable construct. Indeed, attesting to the extent of this negation, Architectural Theorist Alexander Eisenschmidt contends that such a body of architecture read as a 'celebration' that the city

'no longer exists"'... [that architecture is] no longer burdened by that unwieldy thing called 'the city'. ${ }^{41}$

Returning briefly to the beginnings of this chapter, it is no understatement to say that recent, urban architecture has not generally been interested in the project of the city as championed by Aldo Rossi, and other thinkers. Rather, it emanates from the perception that the city - ever subject to urbanisation and indeed, barely conceivable - could not care less about architecture, and is premised on a revised set of priorities which reflect this mutual disregard par excellence: in outlook, modes of practice and projects themselves. ${ }^{42}$

\subsection{A Plain Provocation}

"The discipline's failure to engage the city productively has devastating consequences"

- Alexander Eisenschmidt, 2012, 14

"The subject is always the urban effect: there is no architecture without the city, no city without architecture"

- Bernard Tschumi, Event-Cities, 1994

"The city's principle players - be they developers or policymakers - have come to see architecture as irrelevant"

- Dana Cuff and Roger Sherman, 2012,15.

Alexander Eisenschmidt suggests that this widespread disengagement between architecture and the city has had two detrimental effects: firstly, the trivialisation of the city; secondly, and the diminished instrumentality of architecture in an urban context ${ }^{43}$ - effectively seen to play an ever "minor role" ${ }^{\text {"44 }}$ in an increasingly depoliticised urban context.

\footnotetext{
39 Somol suggest Landscape Urbanism, infrastructural inventions, as part of these 'socially-passive' informalisms $(2012,110)$. (Witte 2012, 77) (Eisenschmidt, 2012, p. 14)(Aureli 2012).

40 (Somol 2012, 110).

41 (Eisenschmidt 2012,14)

42 (Eisenschmidt, 2012, p. 15) (Aureli 2012, 1-15)(Cuff \& Sherman, 2011) Toorn 2012) all note a growing division between intentions of architecture and greater urban directions.

43 (Eisenschmidt, 2012, 14-15)

44 (Helen Castle 2012, 5)
}
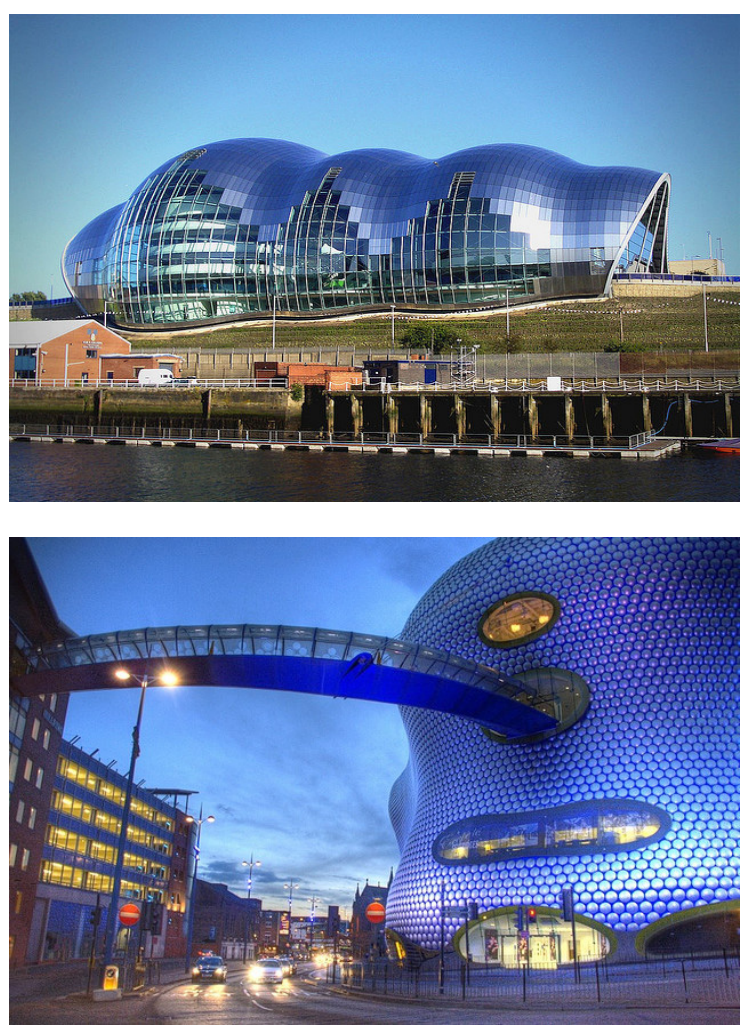

Figures 30-31: Architecture's recent penchant for'formlessness' is widely held to reflect an emphasis on urbanisation's internal, complex fluid and largely a-political forces: 'dictating' architectural form, in what Ron Witte has described as a revamped functionalism. In turn, these projects are understood to affirm that the city'no longer exists' in a political unified or conceivable capacity; as well to affect a widespread disengagement between architecture and the city, more generally.

... it is no understatement to say that recent, urban architecture has not generally been interested in the project of the city as championed by Aldo Rossi, and other thinkers.

Though the latter may amount to a self-perpetuating cycle of cause and effect - architecture's indifference to the city only further reinforcing its diminished potency therein - the former has repercussions that are, in many ways, bigger then architecture itself. Indeed, as seen in chapter 1, the need for architecture to re-assume the city as its project is highlighted by emergent urban realities such as the sub-centre: wherein the comfortable division between architecture and the city (in whichever divergent capacity it now exists) may have propagating and deep-seeded consequences. 


\author{
"The question is not whether one \\ will engage the world, but what \\ the terms of that engagement \\ will be"
}

- Robert Somol, 2012, 110

\subsection{Clawing Back at Lost Territory: re-visiting the city as architecture's project}

This deep-seeded state of neglect between architecture and the city has provoked numerous and varied reactions in recent years, amongst which have emerged proposals to explicitly readdress the city as architecture's project. Echoing the twofold effects of their disengagement outlined above - indeed, for architecture and for the city alike - these proposals are broadly underwritten by a similarly two-fold objective: firstly, to reinstate architecture's role in (and upon) an urban context; and secondly, to reveal the continued existence of the city as political and socio-cultural construct (to uphold the project of the city $)^{45}$. Towards this dual interest, they hold the revision of architecture's political-trajectory and operativity in the city as inextricable from its productive engagement with the city (as an idea). On all counts, the possibility of advancing, catalysing, enacting and influencing the city ${ }^{46}$ is cited as architecture's foremost agenda ${ }^{47}$.

\section{In search of the how (now!)}

This revised agenda has borne an explicit revision of the terms of engagement through which architecture may re-enter, influence and assert its significance in relation to contemporary urban contexts ${ }^{48}$. These do not seek a semiotic framework, nor objective 'rules', but rather mechanisms and operational strategies through which architecture might play a role in shaping not only the city itself, but moreover, an understanding of the city as an idea.

In as much as they respond to a recent state of neglect, these strategies are derived in clear counter to the problematic tendencies outlined earlier in this chapter ${ }^{49}$ - and are by comparison, active, critical and non-curatorial. Yet, they do not simply opt for a return to the principles of their like-minded precursors (Aldo Rossi, Superstudio), despite baring resemblance in objective ${ }^{50}$. Rather, in responding to a contemporary urban condition that is ever more complicated, complex and multidimensional, it is argued that new or revised terms of engagement are necessary. ${ }^{51}$

Given this imperative to 're-invent' the relationship between architecture and the city - and despite the relative consensus over their recent ambivalence toward one another - the means through which a revised engagement might be realised are decidedly varied. Here, as Robert Somol has appropriately remarked, "the question is not whether one will engage the world, but what the terms of that engagement will be $e^{\text {52 }}$. However, three tenets underwrite these proposals more generally, and should premise any further discussion. (figure 32).

\footnotetext{
48 See for example (Castle 2012) (Trummer 2013).

49 Which are seen to have led to the recent disengagement between architecture and the city.

50 That is, to explore and enable architecture's active role in shaping 'the city.

51 This is not to suggest that former methods are redundant, but rather that they must be revised/re-appropriated in today's urban contexts. See (Castle 2012), (Eisenschmidt, Stranger Than Fiction: A Mission See (Castle 2012), 52 (Somol 2012, 113).
} 
Figure 32: Three components to critically re-engage the city. (a) based on the neglect of archifirst half of the chapter, recent first half of the chapter, rece efforts to re-engage the city may be understood to counter these tendencies in three primary ways.
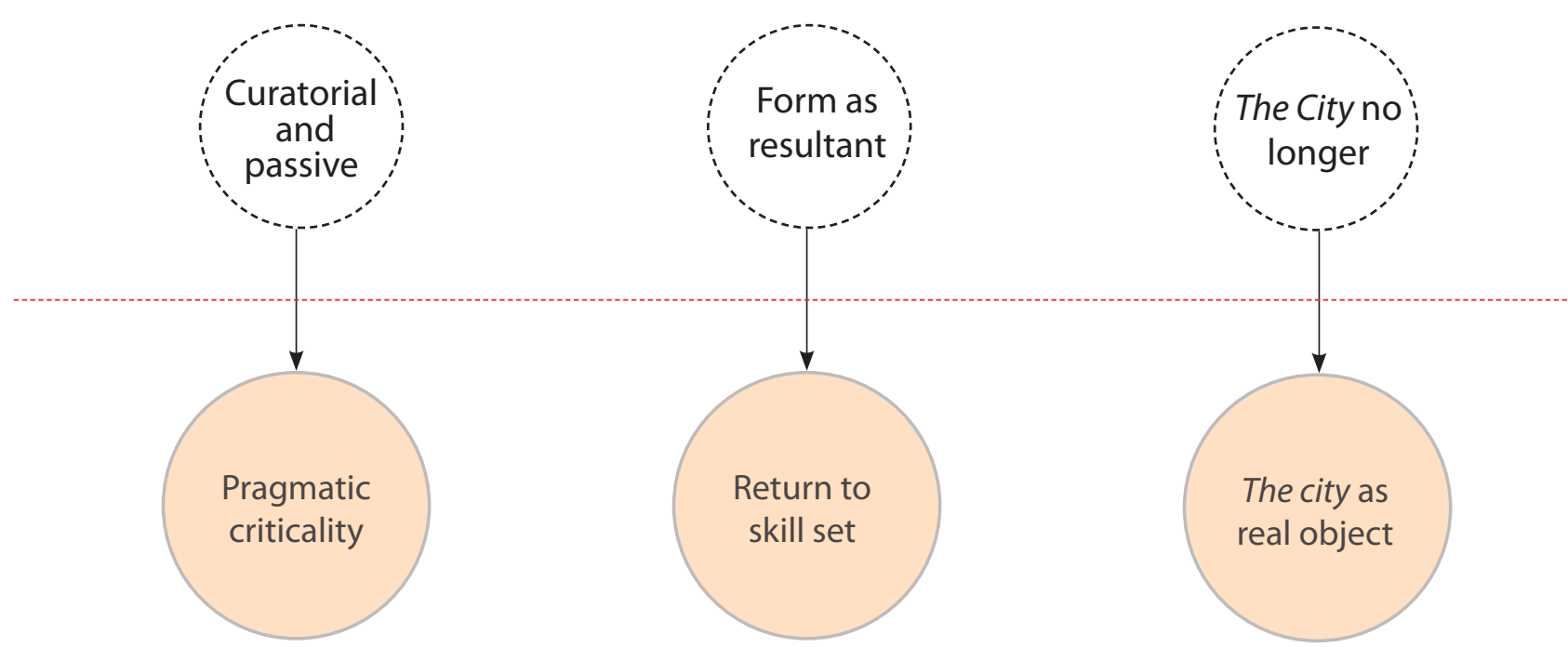

\section{1 - A Critical Pragmatism, in the city}

"The collapse of the grander claims for critical architecture has allowed a space for the return of practical criticism of architectural works, and this, in time, will encourage reflection on how and why we judge some buildings more pleasing than others"

John Macarthur and Naomi Stead, $2012^{53}$

"Criticism may not be agreeable, but it is necessary. It fulfils the same function as pain in the human body. It calls attention to an unhealthy state of things".

-Winston Churchill

The underlying ambition to re-engage architecture and the city in an active, productive dialogue inherently restores a critical agenda for architecture ${ }^{54}$. Here, against the recently-dominant position that architecture cannot be critical ${ }^{55}$ (and should not try), it is seen that architecture can and should 'ask questions' of its context, and, particularly, should engage with larger questions about the city and its transformations ${ }^{56}$. Likewise, whereas these efforts seek re-orientated architectural practices that might actively address or inform radical urban change and a re-politicised urban environment - indeed, not just reflecting, but deflecting urban forces - they are permeated with the

\footnotetext{
53 (135).

54 The potential for architecture to be 'engaged with' the existing is (

1) Moreover, these proposals may be considered fundamentally critical insofar as they actively seek alternatives to dominant practice, on account of its lack of engagement with the city. See (Fischer 2012, 58). 55 Rem Koolhaas' famous statement at the ANY conference in Montreal (1994) that "there is in the deepest motivations of architecture something that cannot be critical" is seen to broadly encapsulate recent. post-critical thought (Fischer 2012, 58)
}

56 (Cupers and Doucet 2009, 1) prospect of architecture's actual urban instrumentality ${ }^{57}$. In plain contrast to a recent emphasis on architecture's facilitation and curation of urban processes, here architecture cites the city as its 'object' -and in so doing, endeavours to act upon something else.

And yet, there is an irrepressible desire for these 'critical' efforts to be rooted in the real, problematic reality from which they emanate - the city; and in this way, an imperative to extract design-based strategies which, in dealing with the built, may indeed allow projects to become truly engaged with this reality ${ }^{58}$. To this effect, and with this external motivation, these proposals might be best explained as a pragmatic criticality ${ }^{59}$ : responding to a need to engage the city, and outlining architecture's agenda accordingly. ${ }^{60}$

In plain contrast to a recent emphasis on architecture's facilitation and curation of urban processes, here architecture cites the city as its 'object' -and in so doing, endeavours to act upon something else.

57 (Eisenschmidt 2012, 14-15)

58 This is significant: whereas, in the 1990s, 'criticality' was understood as synonymous with the 'theory of architecture' itself (Fischer 2012, 57), here it is more synonymous with 'the city.

59 (Macarthur and Stead 2012, 135) discuss a 'flexible', pragmatic criticality, as do (Cupers and Doucet 2009), under the notion of 'agency'. 60 With this overarching urban motivation, architecture's critical agenda is grounded in something external to itself - and must be somewhat pragmatic in pursuing this objective. Rather than subscribing to critical practice associated with internal disciplinary investigations, thes approaches seek'effective' design-tactics: based on what works. 


\section{2 - The City as a Real Object}

"The undermining position taken [on the city as object] over the last two decades has been the primary influence of practice in the city, while the overmining position has been used to theorize out cities. Neither position [...] has considered the object - here, the city - as real"

- Peter Trummer, $2013^{61}$

"[The] city of rampant urbanisation often remains invisible for architecture... [yet] reality for our purposes, has to be understood as the contemporary city"

- Alexander Eisenschmidt, $2012^{62}$

To appropriate Reinhold Martin's oft-cited question upon the aspirations of critical architecture - critical of what ${ }^{63}$ - here, architecture takes the city as the object of its criticality. Whereas recent practice may be seen to have celebrated that the city no longer exists!, these agendas insist upon seeing it as very much existing, and moreover, seek to affirm it as so ${ }^{64}$. This is quite logical: if architecture is to re-establish its engagement with the city, it must understand and address it as a real phenomenon not only as its 'physical domain' or cultural context but as its foremost responsibility ${ }^{65}$ and its project.

Yet, of course, the city does not exist in a one-dimensional or uniform capacity - meaning that understandings of the city (the dimensions of architecture's project) become crucial. Here, the idea of the city, kept largely at bay by the post critical, re-enters architectural discussion with force. As a broad conception of the city as it is, could or should be, it loosely prescribes a reality with which architecture should endeavour to engage ${ }^{66}$. Effectively, the idea of the city becomes architecture's fundamental focal point: eliciting general strategies on the one hand (that is, not specific to a single city); but equally meaning that emerging urban realities, such as the sub-centre, may require different 'terms' of architectural engagement ${ }^{67}$.

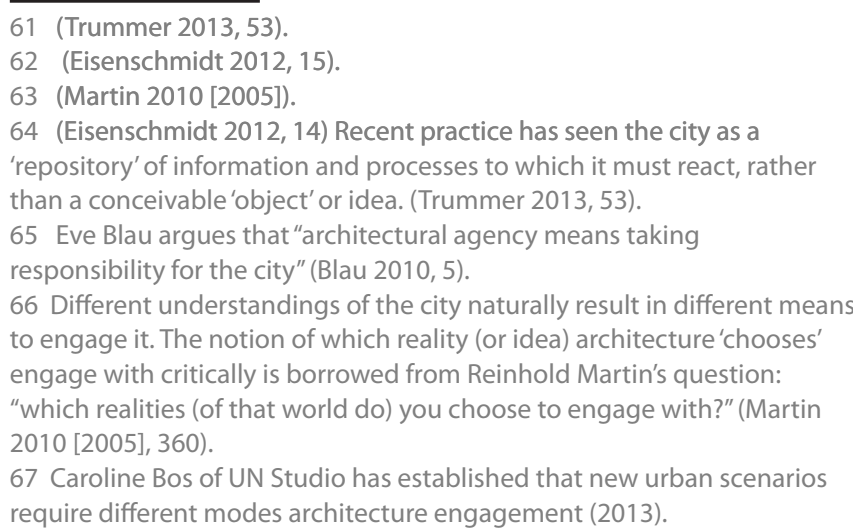

\begin{abstract}
In contrast to the curatorial approaches of recent practice - where architecture's inevitable demand for form may be understood as a'result' of external forces [....] a return to 'conventional skill sets' becomes a strategic device to critically re-engage the city.
\end{abstract}

\section{3 - Re-forming: a return to architecture's conventional skill set}

Robert Somol, formerly one of the post-critical's most fervent proponents, contends that

"if architecture has lost its ability to operate in the world it is not because it has become too self-involved, but because it has not been attentive enough to its own protocols, techniques, and forms of knowledge." ${ }^{68}$

This quote - and its source - not only affirms the perceivedshortcomings of a laissez-faire, a-political approach to 'the city', but in so doing, links architecture's recent urban in-operativity to a lack of disciplinary intentionality. Accordingly, in contrast to the curatorial approaches of recent practice - where architecture's inevitable demand for form may be understood as a 'result' of external forces (or a bottom-up, curatorial approach) - the revision of disciplinary tactics, and often, a return to 'conventional skill sets' becomes a strategic device to critically re-engage the city ${ }^{69}$.

Aligning architecture's critical, political capacities with a revision of its autonomous attributes (internal forms of knowledge) in a way that by no means unprecedented ${ }^{70}$, the intentionality of form, material, tectonics, visual, spatial, aesthetics - deemed 'superficial' by recent practice - here becomes paramount ${ }^{71}$. This amounts firstly, to the position that the architect's primary

\section{$68(2012,113)$}

69 (Castle 2012)(Aureli 2011) (Reiser 2005) (Somol 2012) (Easterling 2011) each posits variations on a renewed formal consciousness in the city

70 Architecture's autonomy has been understood as a pre-condition fo its critical functioning in recent years. See, (Fischer 2012) (Toorn 2005). 71 Neil Leach sees that'visual' tactics have been seldom acknowledged as intentional, in recent practice: but rather, explained according to urban processes. (Leach 2003). 

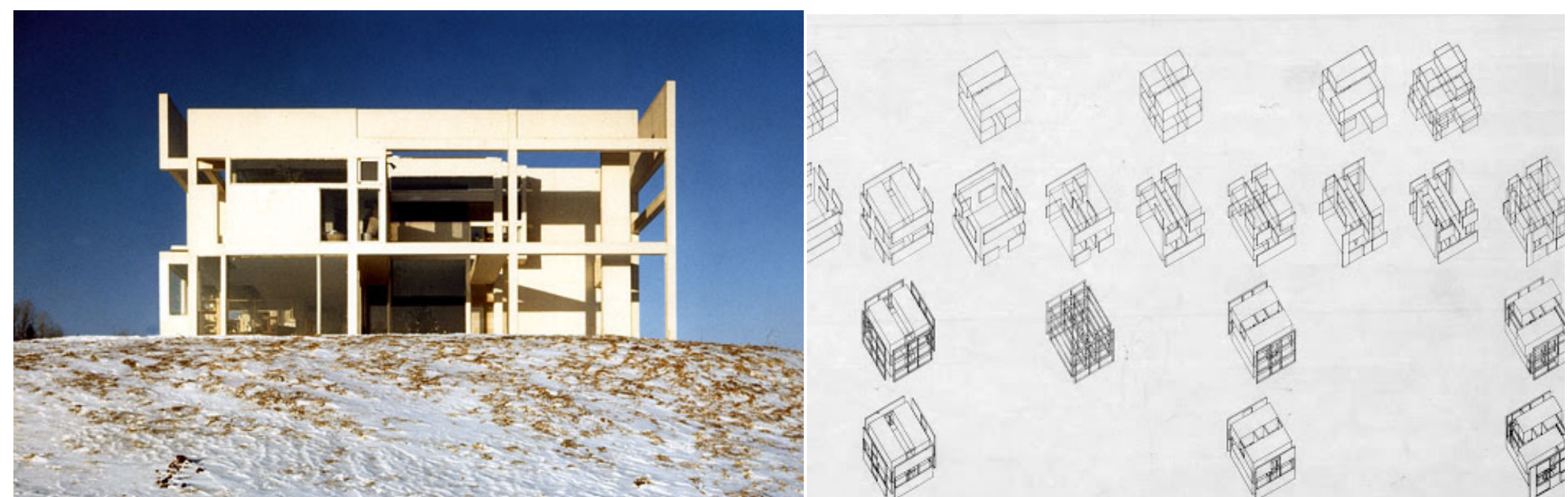

agenda is to investigate the "productive tension between the 21 st century city modern city and architectural form ${ }^{\prime \prime 2}$, and secondly, to the understanding that architecture's urban potency arises outside of its program, and the urban processes it facilitates.

Yet, this renewed formal and material primacy need not signal a return to pure form, restrained aesthetics or the formal 'objectivity' of Architectural Autonomy ${ }^{73}$ (figures 33-34). In fact, rather than looking inwards for its critical capacity, this revised formal agenda looks outwards - to the city - to find its strength (and its activism). In this way, the renewed intentionality and consciousness ascribed to the formal architectural 'object ${ }^{7 / 4}$ is conditioned, achieved and measured by its relationship to the idea of city: and simply, cannot be separated from it. In this regard - and linking back to the first of these three points this renewed formal tendency leans towards the seeminglyparadoxical notion of a purposeful or 'pragmatist aesthetic ${ }^{175}$ : or simply, a formal agenda predicated on an actual, productive relationship to the city.

Figures 33-34: Peter Eisenman, House ii, Hardwick, Vermont, 2005 (and related experiments). Though sharing a simila conviction for architecture's formal potency, Architectural Autonomy was motivated by a disciplinary agenda, and its design strategies were derived accordingly (looking inwards, to the discipline). By comparison, arguments of interest here consider formal strategies in relation to the city.

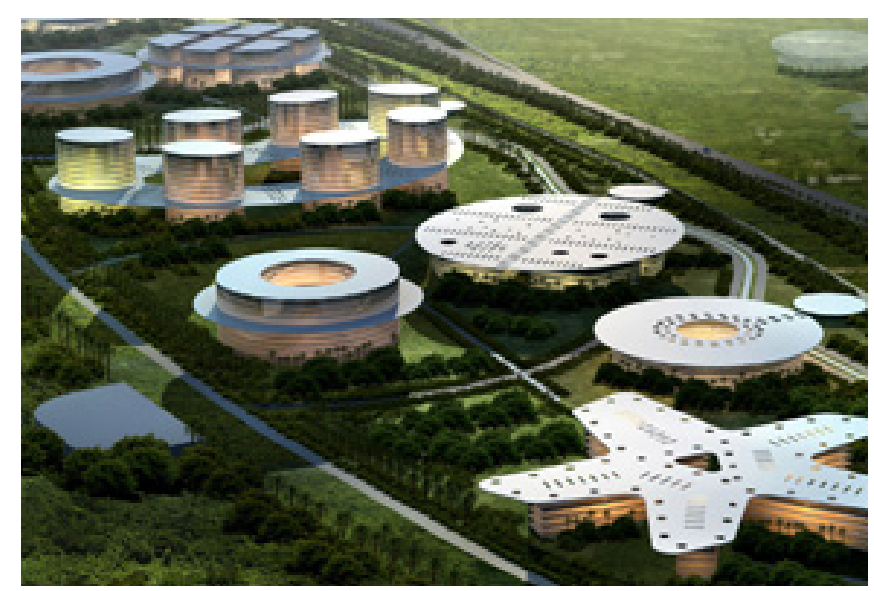

72 (Eisenschmidt, Stranger Than Fiction: A Mission Statement 2012 15). This is notable: whereas in 1999 Rem Koolhaas and Sarah Whiting saw that the task of architecture was to re-invent a'plausible relationship between the formal and the social', and earlier, Michael Hays suggested it was between the formal and the cultural these proposals hold the relationship between the formal and the urban to be of paramount importance. See (Fischer 2012); (Hays 1984).

73 Autonomy holds disciplinary 'interiority' or self-investigation as the only way for architectural to pursue a critical agenda; and is ambivalent about the city as a primary 'project' or motivation for architecture. See about 16. 16) (Eisenman 2011).

74 Recent proposals to rethink 'criticality' following the denunciation of 'critical theory' have re-claimed the agency of the architectural object, countering the decades-long influence of social sciences in architectura production, and pushing intentionality and disciplinarity. For a detailed account see (Cupers and Doucet 2009).

75 John Rajchman challenges the common disparity seen between these terms, and outlines how the 'pragmatist aesthetic' confronts both pure formalism and critical architectural agendas $(2000,115)$.

Figure 35: Bernard Tschumi Architecture, Elliptic City IFCA Masterplan, Santo Domingo, Domincan Republic, 2005. In new urban contexts, the form of the architectural object is 'one concept to start with' as Alexander Eisenschmidt explains (2012,13). These fforts to reengage the city seek a renewed formal potency, fundamentally derived from the city. 

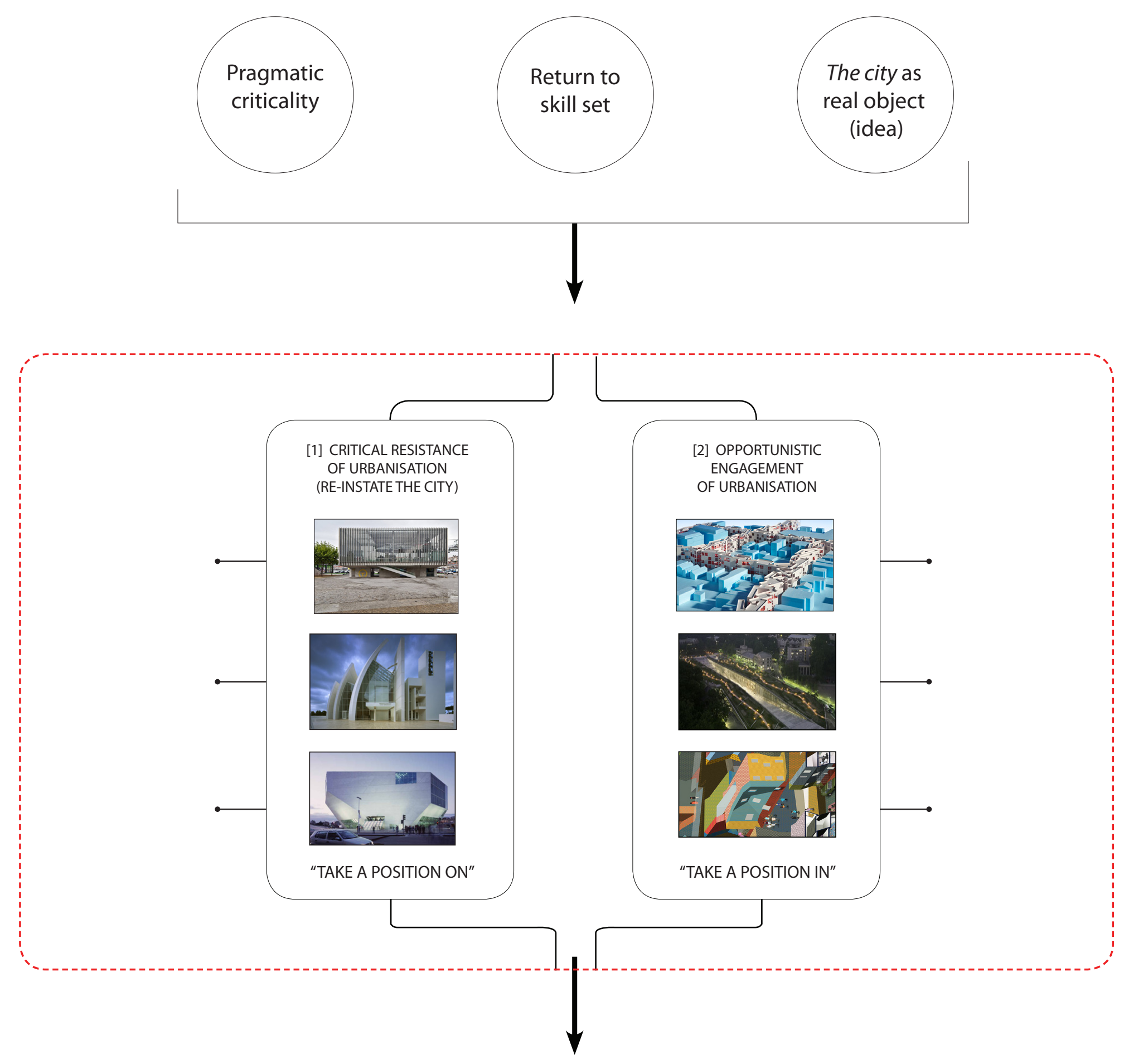

Towards an architecture of the sub-centre: critical opporutnistic
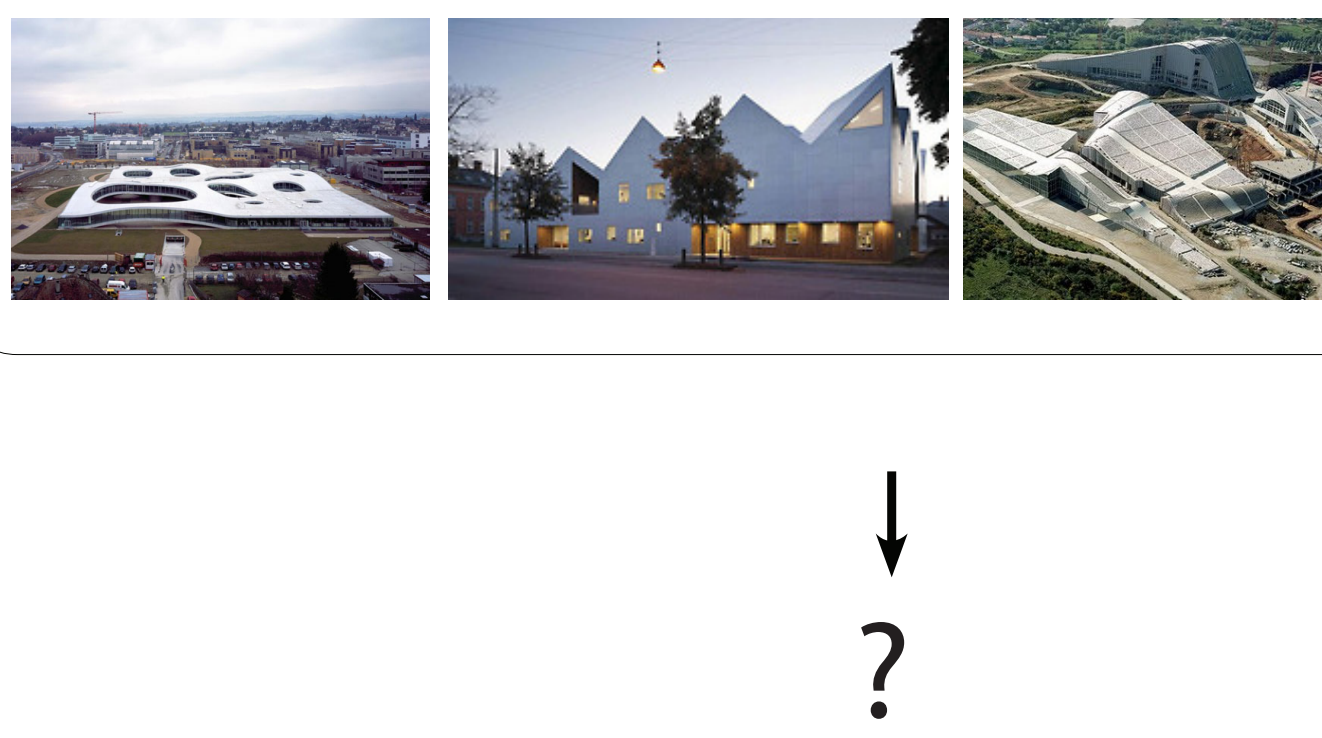

Figure 37: Diagram of Argument. These three main ideas relate to two 'strands' of thought, which can be synthesised in an effort to re-engage the sub-centre as architecture's project. 


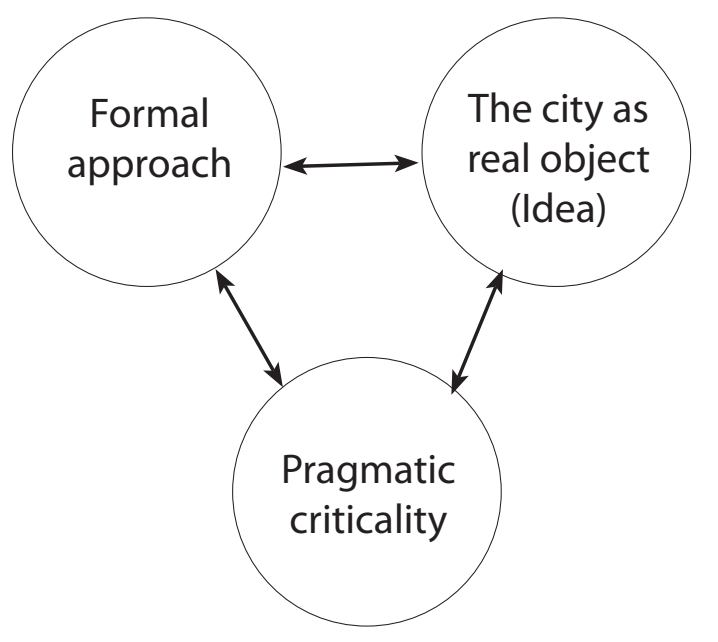

Figure 36: An Architecture city Trialectic. These three terms form the bounds of recent arguments to re-engage the city; and are in constant overlap.

\subsection{Re-igniting the Flame: negotiating a modus operandi}

The persistent overlapping of the three tenets outlined above is not co-incidental. Rather it is acutely representative of recent arguments to let architecture engage the city as its project, wherein each component is, indeed, equally dictating and dictated by the others: architectural form seeking to engage the city; the idea of the city dictating critical imperatives and a critical agenda directing architectural form ${ }^{76}$. (figure 36 ).

This tightly-bound triad provides a framework to discuss two divergent arguments around architecture's engagement with the city: and eventually, their relevance to the emergent subcentre. Despite their aligned agenda to address the city as architecture's project through a renewed formal intentionality, these positions ascribe to differing ideas of the city, and accordingly, procure diverse formal operations to engage it. While neither deals directly with the sub-centre, the disparity between their directives offers a fertile ground on which to negotiate the terms of architecture's productive engagement with this emergent context. (figure 37).

76 It is such that equally interlinked combinations of terms have arisen to explain these arguments: 'Active Form'; 'Pragmatic Aesthetics'. 'City as Political Form' for example.

\subsection{Terms of Engagement 1: re-instating the city through formal resistance}

\section{An Idea of the City: the urban archipelago}

The first of these positions, championed by esteemed academic and practitioner Pier Vittorio Aureli of the Architectural Association, argues to revisit architecture's productive engagement with the city through a formal resistance of urbanisation - here understood as the antithesis of 'the city'. This argument upholds an idea of the city that is fundamentally political - a collage of parts in constant confrontation, and a space fundamentally of public relations and articulated difference. Based loosely on the greek concept of polis, this idea of the city is approximated by the Urban Archipelago: a conceptual model of the city incumbent on principles of separation, the articulation of different parts, countercomposition, and, governed by a series of relations - or, a city of unique heterotopias ${ }^{77}$. (Figure 38)

The notion of Island Urbanism presented by this paradigm equates the intrinsically political nature of the city with its formal character, insofar as the built objects within the city (its form) are seen as a critical device to 'frame' and actively negotiate

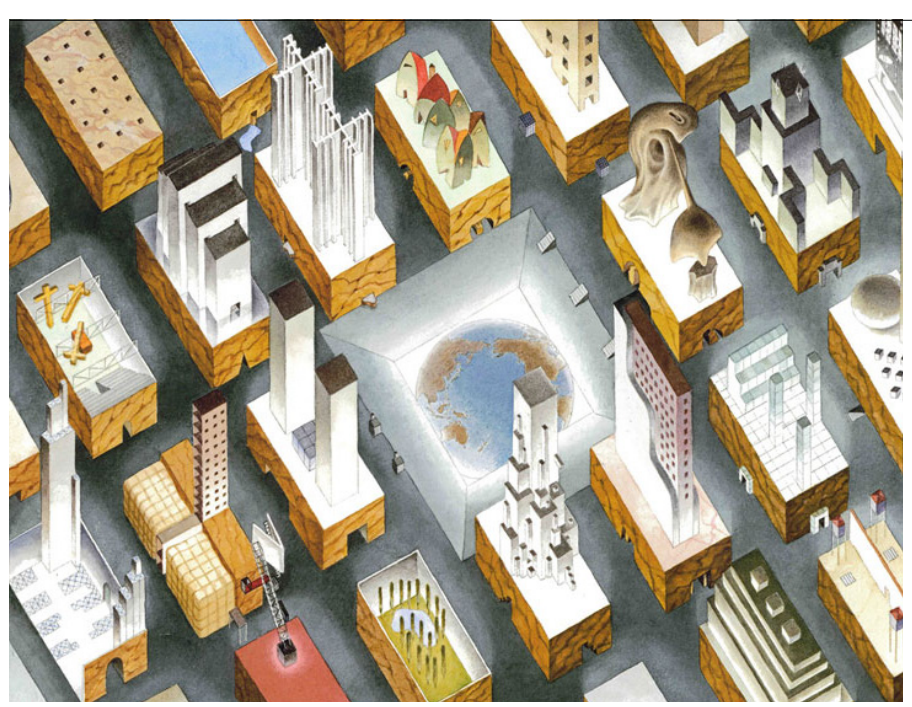

Figure 38: Rem Koolhaas and Madelon Vriesendorp, The City of the Captive Globe Revisited, New York, 1994. The Idea of the City is understood as an Urban Archipelago: made up of different, contrasting parts which negotiate and frame the public space between (and are separated by it). The individual blocks (buildings or islands) confront the'smooth, (buildings or islands) confront the'smooth, formlessness' or urbanisation through their separation and establishment of limits, acting as a 'city within a city'. In this way, the architectural/urban form enacts the idea of the city as a space of political differentiation.

77 Aureli discusses the urban archipelago paradigm in depth. See (Aureli, The Possibility of an Absolute Architecture 2011). 
The possibility of reinstating the idea of the city as a political project - of separation, confrontation and difference - becomes synonymous with actively resisting the amorphous nature of urbanisation: re-establishing the limits it has relinquished.
IF A O (HOUSE) IS LIKE A $\%$

(SMALL CITY/AGGREGATE OF BUILD-

$\quad 00$
INGS), AND A
0000 (THE CITY), IS
000

Like a $\bigcirc$ (Large house/Object),

THEN O (THE HOUSE) CAN BE SEEN

As A 80 (SMALl aggregated

OBJECT) AND THE 000 0.0 (THE

CITY) AS A

OBJECT) ONE.
Figure 39: An indexical relationship between architecture and city allows the building to be understood as an urban microcosm. This is used by the first of these positions, as a means to re-engage architecture with the idea of the city. the open, smooth, space between the different parts of the city - in effect, enacting and exacting conflict, confrontation and 'the political'78. For want of a direct metaphor: the islands and the sea of the archipelago are in necessary and constant negotiation, forming a veritable tension between public and private and attesting to an idea of the city as such. To this end, the archipelago does not represent a literal understanding of the form of the city (as a physical entity), but rather, offers a conceptual understanding - indeed an idea of the city fundamentally based on political, public and confrontational space $^{79}$.

Citing familiar dichotomies of political-economic; publicprivate, modern urbanisation's homogenising, amorphous and privatised economic forces are easily problematic for this idea of the city. Where these forces have engendered formless, sprawling, and limitless cities (both at the scale of urban structure, as well that of built form), they have essentially displaced the fundamental 'city' ideals embodied by the archipelago: differentiation, composition and the city as political, public space ${ }^{80}$. Specifically, as urban form has dissolved 'limits' (physical), it has also dissolved (metaphysical) limits: power relations with the city, for example, by invoking innocuous conditions of 'privatised public', and economic-political - to the extent that the city is characterised by a ubiquitous grey area.

In short, the possibility of reinstating the idea of the city as a political project - of separation, confrontation and difference - becomes synonymous with actively resisting the amorphous nature of urbanisation: re-establishing the limits it has relinquished. ${ }^{81}$

78 The political is understood to arise here not through conflicting parts themselves, but rather, in the space between them. In this way, Aureli argues that the city is the 'most explicit index for power relationships (Aureli, City as Political Form: Four Archetypes of Urban Transformation 2011, 32)

79 These ideas are mainly taken from Pier Vittorio's Aureli's concept of an Absolute Architecture. For an elaborate explanation of these ideas, see (Aureli, The Possibility of an Absolute Architecture 2011). 80 Modern urban dictates are often linked to a lack of political (and cultural) urban identity (Gyurkovich 2011) (Aureli, The Possibility of an Absolute Architecture 2011).

81 This is a dense version of Aureli's argument on the topic. See (Aureli, The Possibility of an Absolute Architecture 2011).

\section{Architecture's Agenda: a city within the city}

Architecture's urban agenda here is configured according to a unitary view of architecture and the city that dates back as far as Alberti's famous dictum the city as house / house as small city. Bound in an 'indexical' relationship to its urban context; architecture is understood as a city-within-the-city ${ }^{82}$ : aspiring to become an urban microcosm by embodying those same qualities identified in the archipelago: separation, counter-position, composition and agonism ${ }^{83}$ (figure 39). Not incidentally, these are qualities that architectural form - in its inherent state - is understood to naturally possess: a composition of parts, a set of relations, an embodied state of difference. Accordingly, this unitary interpretation of form and city becomes a dual basis to argue that a renewed 'Formal' consciousness is a pre-condition for architecture's engagement with the city, as a political project ${ }^{84}$.

Notably, what amounts here to engagement on the one hand, amounts to resistance on the other. Where urbanisation's main function is the"destruction of any limit, boundary, or form that is not the infinite, compulsive repetition of its own reproduction" ${ }^{\prime \prime 5}$, the possibility of confronting it arises in architectural forms' ability to pronounce its inherently 'finite' nature and to enact limits and 'stoppages' that may operate against the smooth, amorphous space of urbanisation ${ }^{86}$. In effect, the architectural object engages the city through formal resistance

\footnotetext{
82 This concept was famously developed by Oswald Mathias Ungers and colleagues at Columbia University, in 1977, and has been highly influential in recent architecture-city thinking. See (Ungers 1977). 83 Interestingly, whereas Architectural Autonomy saw the architectural object as a disciplinary index (triggering geometric progression) here, it is rather an index for the idea of the city (triggering urban progression, effectively). See (Eisenman 2011)

84 This argument unequivocally disregards 'external elements' such as programme, functional, historical; as well as larger scale infrastructural manipulation of the city - all as having a limited potency upon the city; and is the underlying thesis of Aureli's text (The Possibility of an Absolute Architecture 2011).

85 (Aureli, The Possibility of an Absolute Architecture 2011, 16). 86 That is: where urbanisation is seen to have procured an architectural formal separation and confrontation.
} 


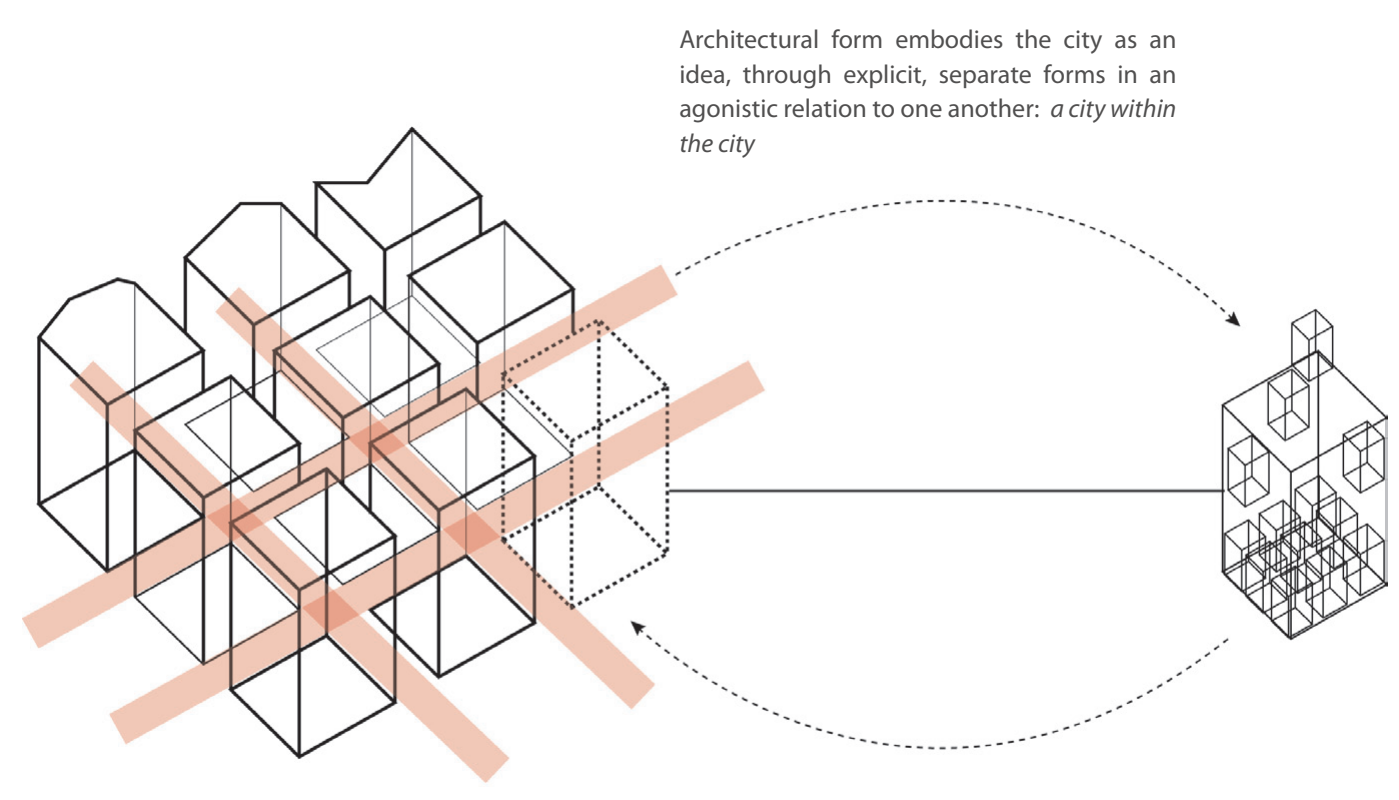

By internalizing or formalizing the city, it becomes a seperate yet integrated part of the city (and thus, reinforces it as a whole).
Figure 40: A Part-to-whole Relationship. Architecture's indexical relationship to the city allows the architectural object to be read as a city-within-the-city. By embodying the qualities of the urban-archipelego, through its form, the architecutural object re-inforces the idea of the city as political space. and by considering itself a "formally distinct and functionally autonomous miniature city" ${ }^{187}$ : simultaneously assuming the values of the archipelago, and asserting itself within it as a formally unique, autonomous and separate entity (figure 40). Pursuing this agenda, architectural constituents in the city may become - hypothetically -'absolute' ${ }^{\prime 88}$.

\section{Towards Formal Operations and Strategies: enabling architecture's political project}

The strategic making of architectural form (or Form) here fundamentally aspires to two things: firstly, to articulate the architectural object as an index for the city, and secondly, as and separate 'piece' of the city. With this agenda, the processbased techniques of recent years, with their propensity for fluid, informal, and a-formal outputs, become immediately problematic - and as such, provide a springboard for response. These urban forms are equated with architecture's urban and political impotency - negating architecture's finite, separate 'formal' nature, and in so doing, eliminating its provision of boundaries and 'limits' which can frame the space of the city. (figure 41) In direct contrast, urban forms and typologies understood as resolute, separate, resistant, singular, formal, geometric and comprised of limits and 'stoppages', are necessitated to re-engage the city as a project.

87 (Eisenschmidt, The City's Architectural Project: from Formless City to Forms of Architecture 2012, 24).

88 This concept of 'Absolute Architecture' is the hypothesis of Aureli's thesis, and refers to the 'possibility' of architectural form becoming constitutive of the idea of the city: interpretable as an index for the city as a whole. Though described by the author as ultimately impossible aspiring towards this possibility may allow architecture both to

understand the idea of the city, and advance it as a project. See (Aureli,

The Possibility of an Absolute Architecture 2011, x).
Several formal operations can be offered towards this goal. Primarily, these allow architectural form to possess - and to articulate - qualities of separation, confrontation and, integration: both internally (between parts of the building) and holistically (in its entirety).

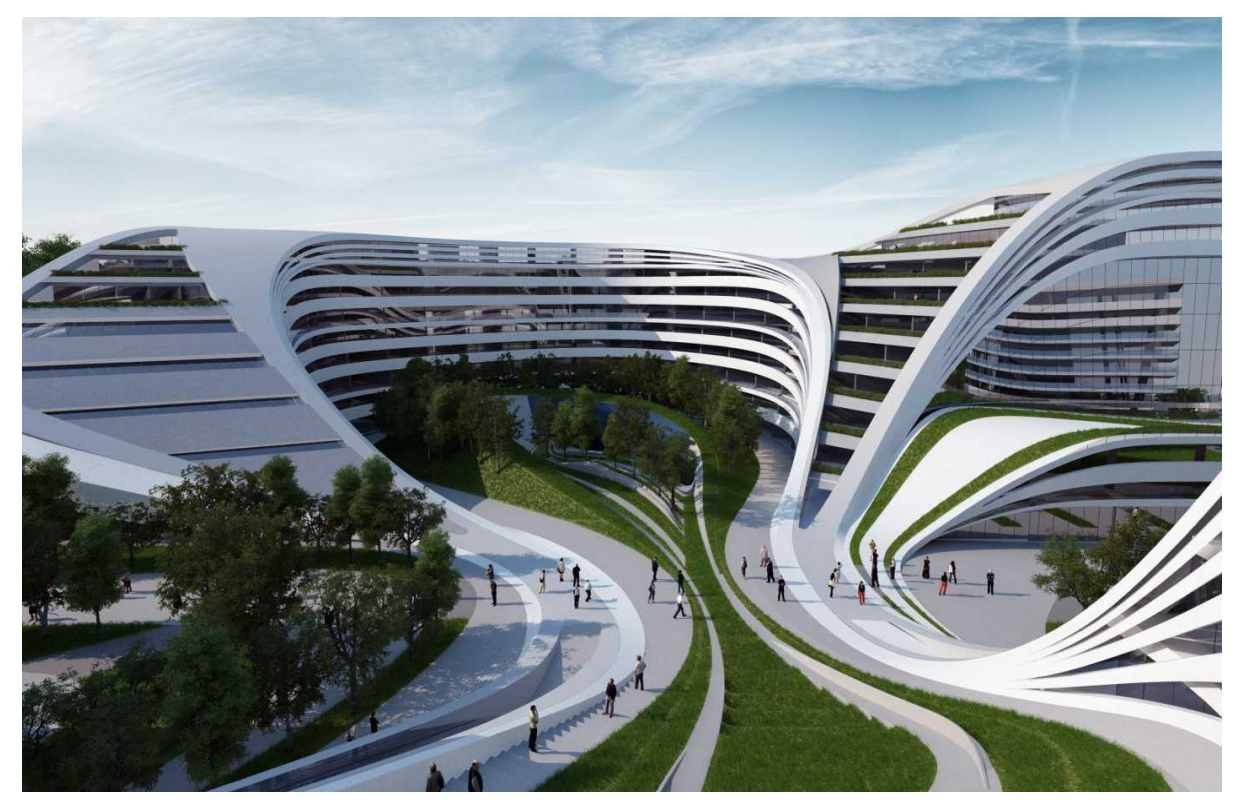

Figure 41: Reinforcing Urbanisation's Smoothness. The fluid forms of recent years are seen to oblige the 'limitless' space of urbanisation. Such forms provide no formal 'stoppages' or boundaries, failing to articulate themselves as a'piece' of the city, or to actively frame the public space around them. Based on a unitary interpretation of architecture and city, interpretation of architecture and city, the city as a political, relational space. 


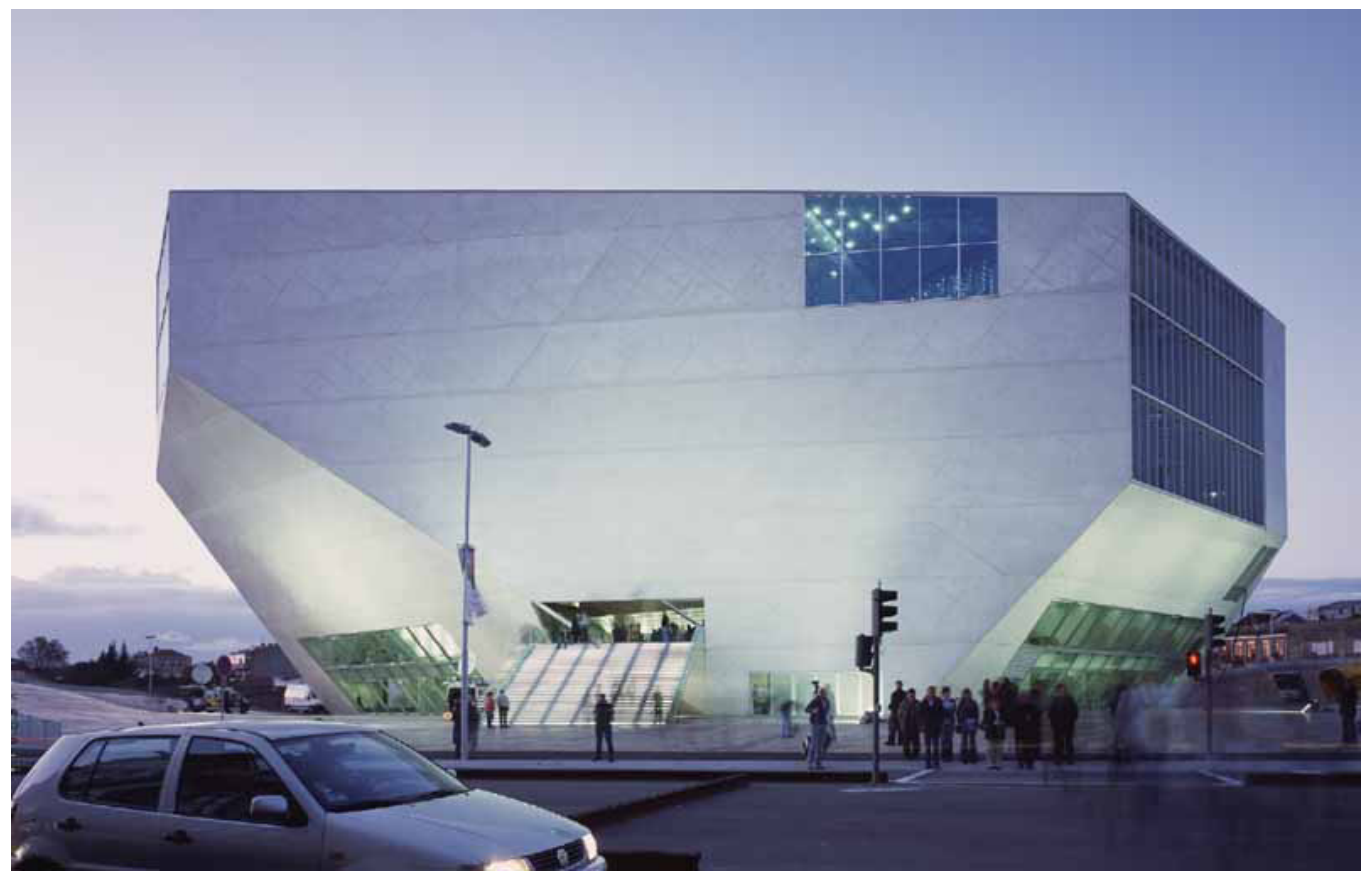

Figure 42: El Casa De Musica (2005) OMA, Porto, Lisbon. The building exemplifies the idea of 'stoppages' and separation: its formal specificity allowing a clear articulation of limits. In its urban context, the building clearly pronounces itself as a resolute, finite object: separated from the space around it, and yet giving form to it as it does so. In this way, the project delivers those qualities upheld as 'resistant' and engages with an upheld as city as political, confrontational space.

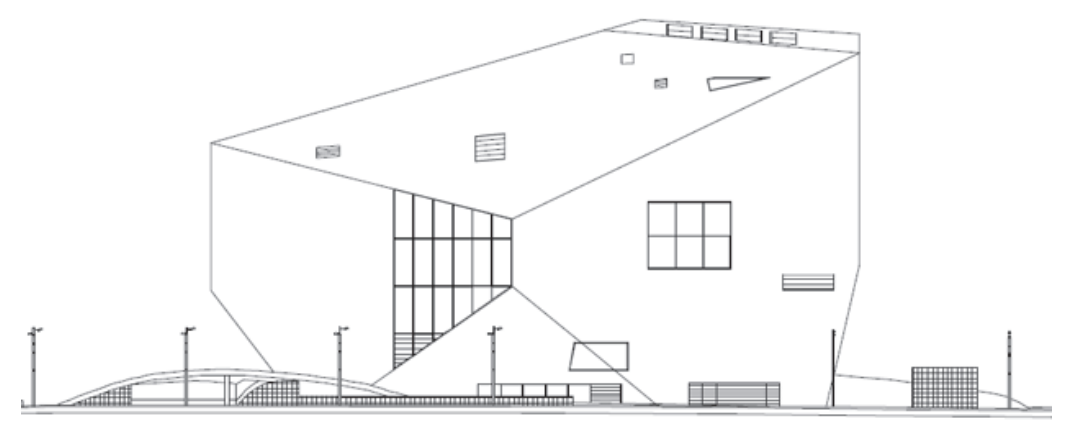

\section{1 - Separateness: articulating limits, stoppages and difference in the infinite field}

"Through its act of separation and being separated, architecture reveals at once the essence of the city and the essence of itself as political form: the city as the composition of separate parts"

Pier Vittorio Aureli, $2011^{89}$

In being resolutely itself, and [...] differentiating itself from its surrounds - the architectural form attests to the logic of the archipelago: indeed, it becomes a part of it.

Figure 43: An elevation showing the articulation of edges and strong geometric language. The building envelope's finite nature allows it to differentiate itself from its surrounds, and sit resolutely as a'piece of the city'.
The articulated separateness of the architectural object-form enables it to both position itself in the city (as an individual, 'singular' piece simultaneously framing and framed by the smooth space around it); and, at a more metaphysical level, to enact the resistance of urbanisation through the articulation of limits or indeed, stoppages ${ }^{90}$. In being 'resolutely itself' ${ }^{\prime 91}$ - differentiating and distinguishing itself from the open space around it - the architectural form attests to the logic of the archipelago, by becoming a discrete part of it. As it reinforces the whole through its state of difference (a uniform condition across the city), the separated, built form constructs (and is constructed by) the space of the city: enabling a dialectical relationship between the open space of the city and the built form: enabling the city's political character.

8915

90 Aureli argues that 'stoppages' expressed in architectural form can resist the flows of urbanisation (Aureli, The Possibility of an Absolute Architecture 2011)

91 Aureli uses this turn of phrase to describe architectural forms that in their individualised state, are separated from their 'other': the space of organization, city, and its government. (Aureli, The Possibility of an Absolute Architecture, 2011, pp. 1-5) 


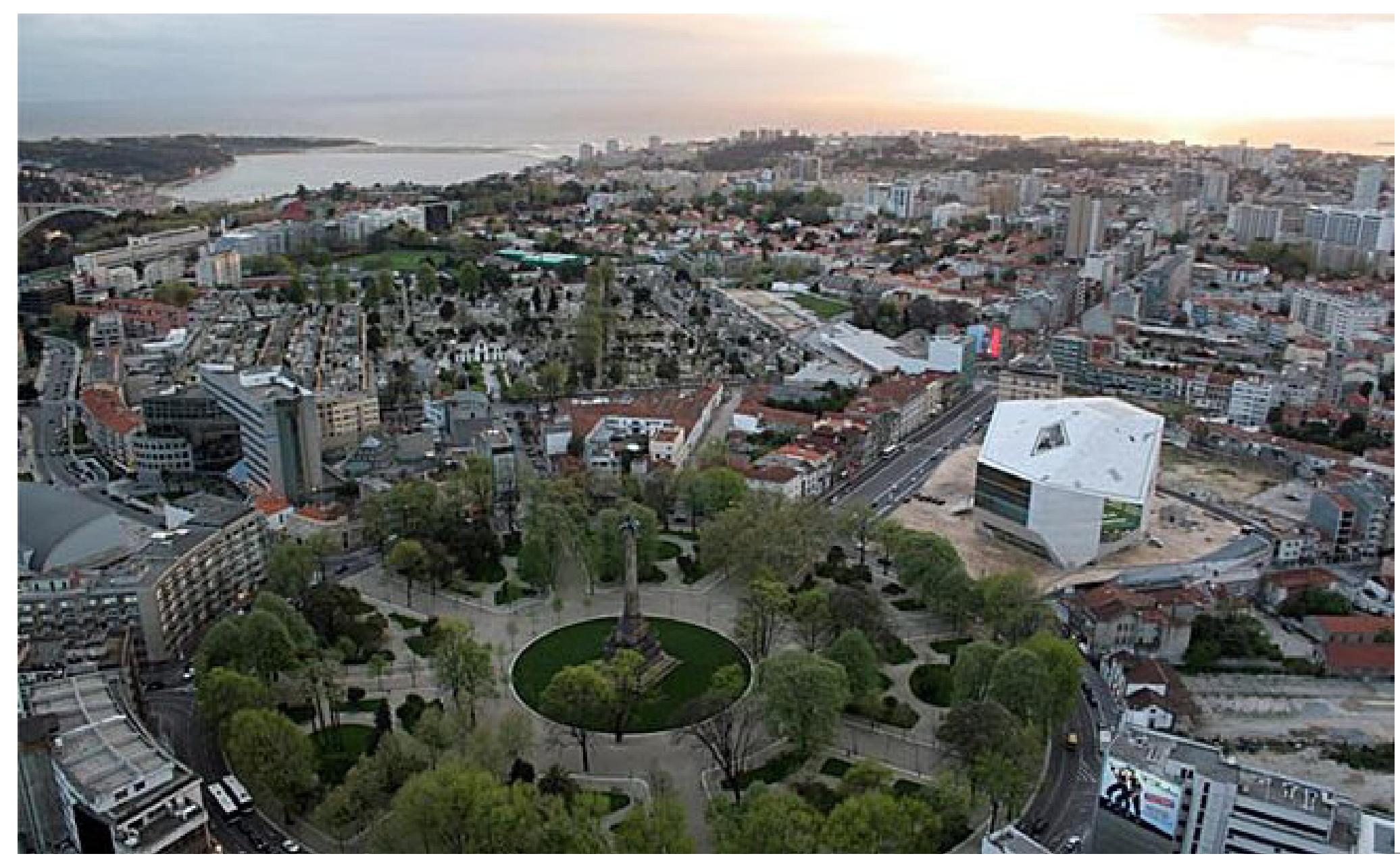

Though it is possible to argue that all buildings are to some degree 'separate', it is the intentional and expressive articulation of separateness, and equally, the dialectical relationship with the city's open space it enables, that is of vital importance here ${ }^{92}$. To return, briefly, to the fluid forms above: (fig--): their unarticulated, innocuous edges indisputably prevent them from being read as resolute and individual architecture objects; and, likewise from ascribing limits or 'form' to the public, city space around them. While these may purport to confront other contextual concerns - they cannot be seen to confront nor ascribe any limits to urbanization, but rather allow a blurring of differences that only facilitates its amorphousness (and its a-political predilections.) (See figures 42-45)

... it is the intentional and expressive articulation of separateness, and equally, the dialectical relationship with the city's open space it enables, that is of vital importance here.

92 In fact, this is intrinsic to this argument: the separateness of architectural form is its 'natural'state of being (and needs to be attended to more rigorously), as Peggy Deamer suggests in her reading of Aureli's text (2011).
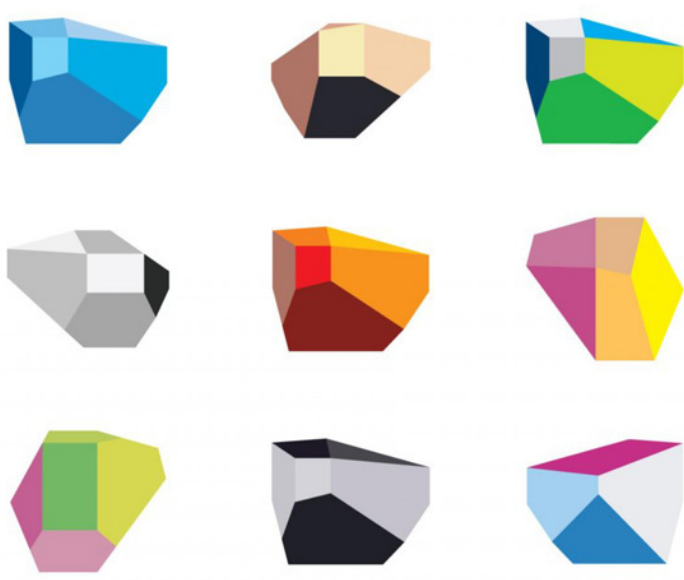

Figure 45: formal

explorations depicting

the building as a seperate

architectural object-form

- establishing limits as a

means to engage the city. 


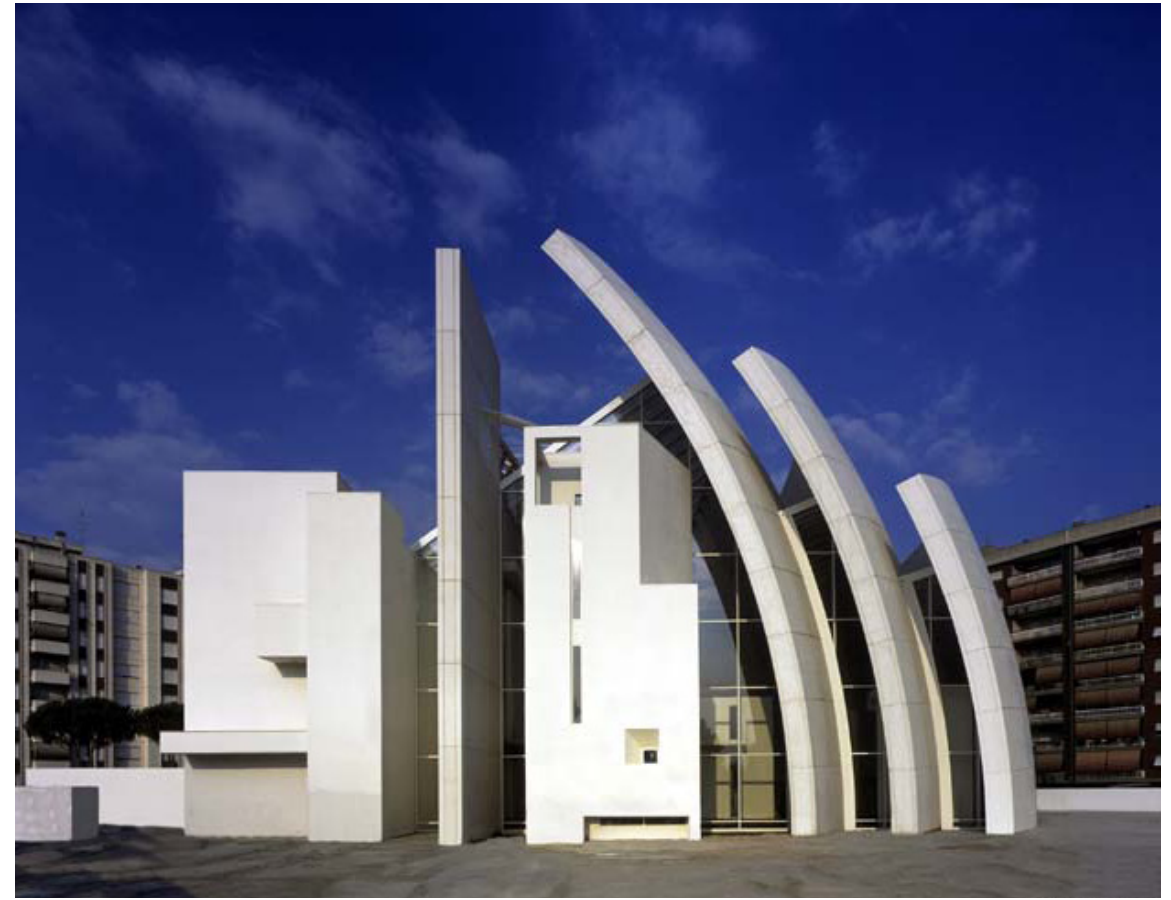

Figure 47: Richard Meier and Partners, 2003, Jubilee Church, Rome, Italy. Located in a suburb outside of central Rome, the build ing has a strong presence through its formal compositioning; that tends indeed towards city-ideas. The three carefullly-proportioned dramatic concrete shells are visible seperated by the glass between them.

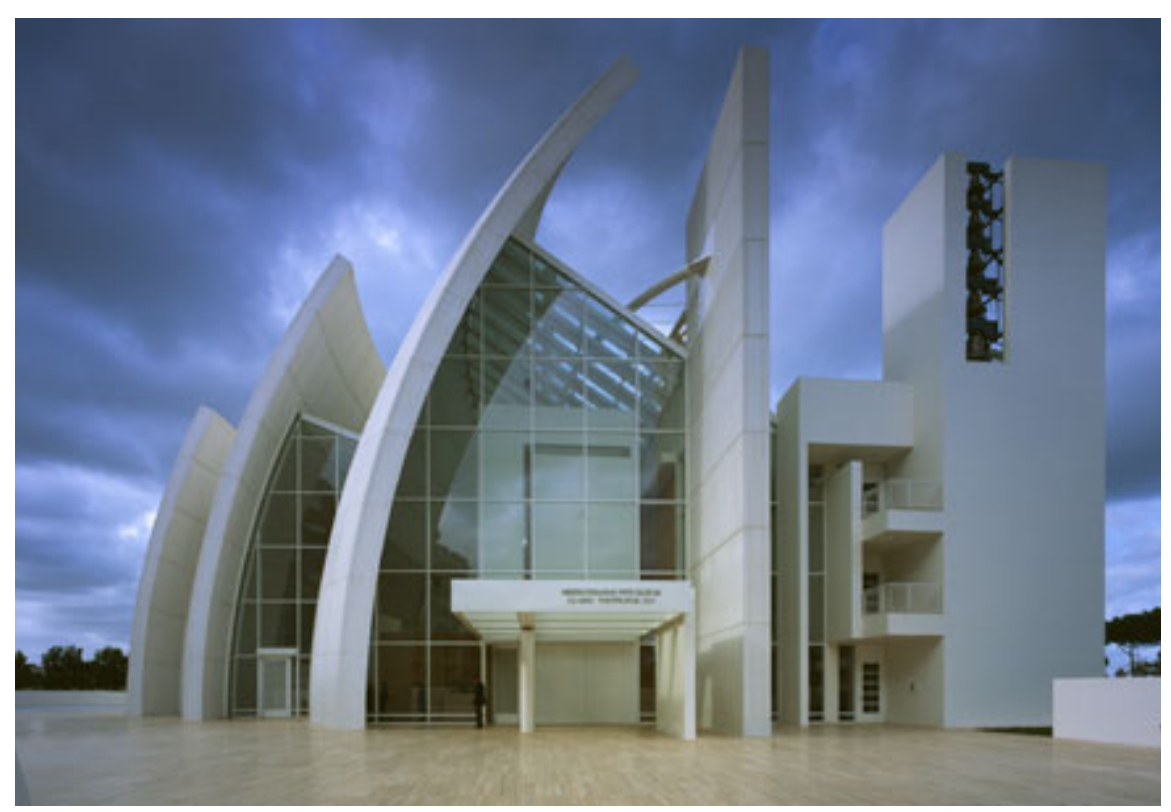

Figures $48+49$ : through its discrete expression of different parts and the agonistic relaitonship between them, the building embodies a compositional tension. While the elements are articulated as seperate, they are also intergrated into the whole through the specificity of the overall composition. Below: elevations.

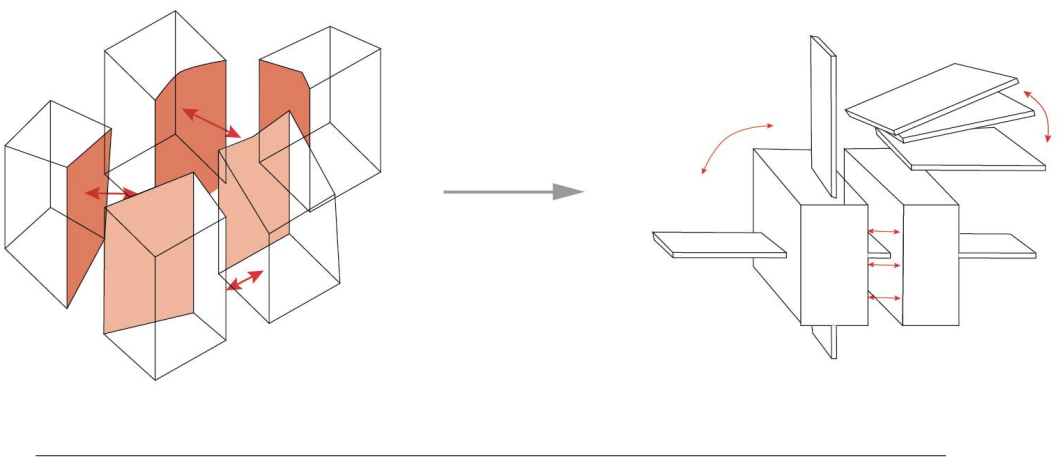

Figure 46: Understanding the idea of the city as a composition of different parts in tension (conceptually and physically) directs a similar compositional tension between different parts of the architectural form

\section{2 - Counter-Composition of [separate] Parts: articulating agonism and a state of difference}

The second strategy alludes directly to indexical, notion of architecture as a city-within-the-city, seeing that architectural form should internalize the agonistic relations that define the city as a political, public, and relational realm: a space of different parts held in dialectical tension. Fundamentally, this requires attentiveness to the formal composition of different parts within the architectural object, and profits a state of a tension, agonism ${ }^{93}$ or counter-compositioning between them. (figure 46).

More specifically, this infers an organisation of architectural volumes (or elements) that is both cohesive and countering; agonistic and conversational - enabling them to be both separate yet integrated within the larger whole ${ }^{94}$. The antithesis of this agonistic condition lies in a certain complacency of different parts (that is, rather than in the absence of different parts entirely). This formal operation comes into play especially when it is confounded with the need for the separateness of the overall object, outlined above; the former requiring discrete elements, the latter a unified, resolute whole: two principles not opposed, but not always easily reconciled. (figures 47-49).

...this requires attentiveness to the formal composition of different parts within the architectural object, and profits a state of a tension, agonism or counter-compositioning between them.

\footnotetext{
93 Importantly, agonism differs from antagonism, in that the former s predicated on something else (does not make sense in isolation), whereas the latter is an internal, independent quality (can happen in isolation)

94 Notably, this is adopting the same principle of separateness (discussed above) on a different scale: reflecting the 'indexical' basis of this argument.
} 


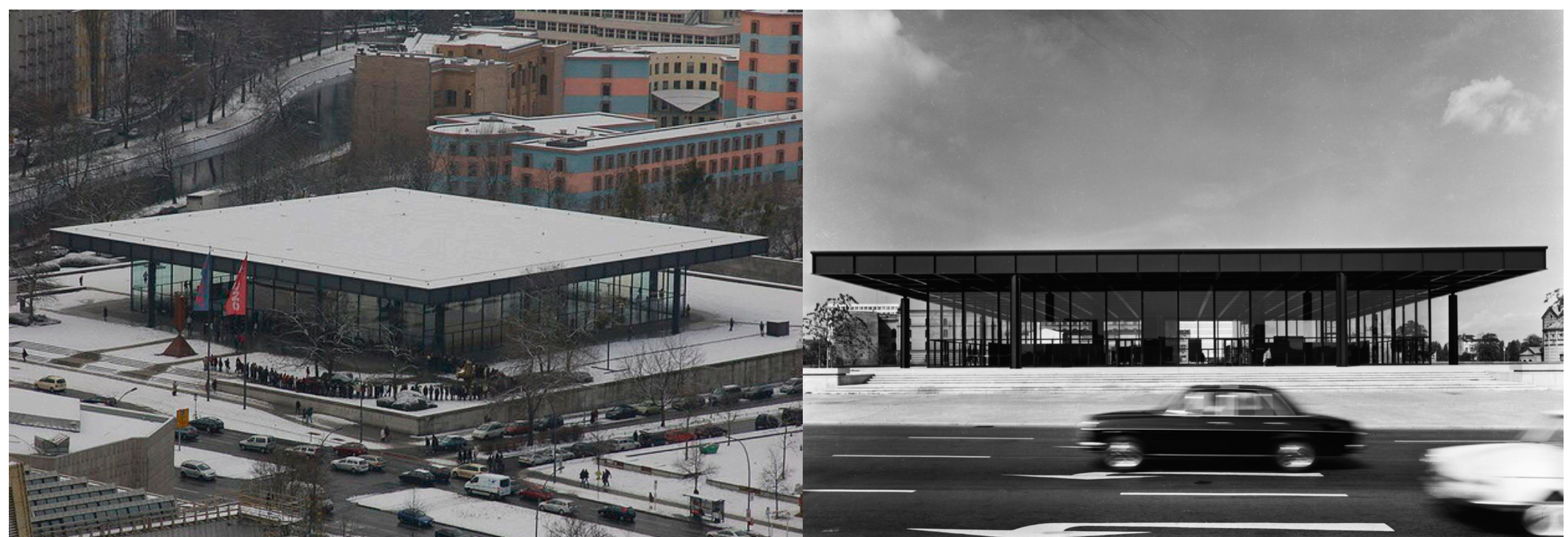

\section{3 - Integration through Typological} Advance:

Where the latter two reflect objectives of separation and confrontation, the third strategy is more concerned with how the built object may reinforce the consistency of the archipelago: as a field of difference, but a field nonetheless. Here, typology is outlined as formal means of assimilating (or integrating) with the city and affording it conceptual unity across any scale $e^{95}$ - while still enabling distinct, seperate parts within it.

Of course, typology has often been central to inquiries into architecture and the city: understood as an historic resource; and a springboard for formal innovation, and a point of continuity, and in this way, has permeated attempts to address the city as a project ${ }^{96}$. Here however, the strategy of typology is primarily seen as a means to enable a degree of (formal) consistency within the city at large, as well as to enable site-specific interventions within it $^{97}$. To this end, it elicits forms that resound with the urban context (somewhat objectively), but also references the continuity of the city at large (as a continuous field). In effect, the variance enabled by typology (that is, different types), is what enables difference within the city on the one hand: and offers coherence and unification on the other.

To this end, typology becomes a means to engage the city, as a direct tactic of formal integration. Mie's Van Der Rohe's frequent use of the plinth is heralded by Aureli to be exemplary here: a typological gesture that serves to articulate the building as an integrated 'piece' of the city (yet, allows it to assert its separateness no less) ${ }^{98}$. However, more generally, typology affords here a level of site-specificity and means of urban continuity, which the previous two principles are less concerned with. (figures 50-2)

\footnotetext{
Aureli does not suggest the 'city' itself has limits - despite the fact that it is comprised inherently of limits (internally). Rather, he sees it as a field of difference that could theoretically be infinite, but must retain those same political qualities nonetheless.

96 The use of typology has often reflected a fascination with the historic city, for example: Aldo Rossi's Architecture in the City is especially relevan in this regard (1982). In a more contemporary setting typology has been widely explored as a platform for formatinnovation, and is widely regarded widely explored as a plaforn for forma as a tactic towards progressing the city as a project (lee and Jacoby, 2011 97 That is, insofar as typology naturally deals with an historically and culturally-inflected relationship to site

98 (Aureli, The Possibility of an Absolute Architecture 2011).
}

Figure $50+51$ : Mies Van Der Rohe, Neue National Gallery, 1968, Berlin. Aureli notes Mie Van Der Rohe's frequent use of the plinth as urban'type' as a me the plinth as urban'type', as a means to both integrate and separate the architecture from the city, and to confront the space of urbanisation.

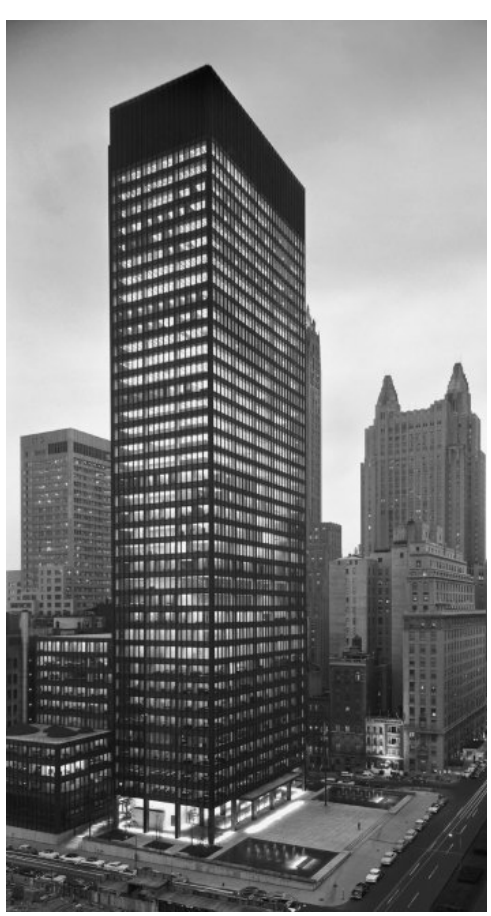

The variance enabled by typology is what enables difference within the city on the one hand [...] and offers coherence and unification on the other
Figure 52 : Mies Van Der Rohe, Seagram Building, 1958, New York. The use of the plinth as an urban type enables a point of formal consistency within the city. The architectural object can assimilate itself with the greater logic of the city as a continuous field of difference effectively becoming a 'piece' of the archipelago.


...while the principles put forward may appear to suggest autonomous forms at times, the critical emphasis on urban integration reflects the fact that these approaches are ultimately concerned with the city as a political, public project - rather than an internal disciplinary investigation

\section{In Summation}

In spite of its theoretical underpinnings, the strategies offered - based on a part to whole embodiment of the city and formal resistance - are quite readily applicable to a design context. Moreover, while the principles put forward may appear to suggest autonomous forms at times, the critical emphasis on urban integration reflects the fact that these approaches are ultimately concerned with the city as a political, public project - rather than an internal disciplinary investigation ${ }^{99}$. Theorist Jeff Diamanti sums this up perceptively:

"The concept's architectural valence is bound between a dialectic of architecture's historically specific struggle for autonomy and its tendency towards urban integration"100

Furthermore, while these formal 'strategies' are in some ways self-evident (indeed, concerns such as composition and separation seeming common considerations in dealing with the architectural object per se), this is in fact the very point of the argument: that a renewed formal consciousness is tantamount to architectures' re-engagement with the city (rather than any further need to re-invent the wheel). In this way, it is the revised attentiveness and intentionality around these formal strategies, and of course the specificity with which they are executed, that may allow architecture to take a position on the city, and in so doing, push back upon it as a deeply political and public idea ${ }^{101}$.

99 This indeed reinforces the difference between this position and that of Architectural Autonomy, which are often confused but are in fact quite distinct. For Aureli, these autonomous forms are a means to an end, effectively.

100 (Diamanti, 2012).

101 That is, intervene, critically, on the city as an idea, and thus advance it as a project. 
Rather than seeing urbanisation and the city as antithetical, this position sees that the city does very much exist - but in a re-configured and ever-urbanised state, and one that often lies unrecognised.

\subsection{Terms of Engagement 2: re-evaluating the city through a critical opportunism}

"New possibilities for experimentation are discovered at the nexus of architecture and the city"

- Helen Castle, $2012^{102}$

"[In the City], one could detect a lot, but more importantly, one can dream up and imagine all sorts of things"

-Walter Benjamin, radio broadcast, 23 February 1930.

"On the one hand an openness towards the city is needed that enables us to see latent possibilities in the most unlikely environments. On the other, we must invent new protocols and operations that incorporate the intelligences of the city so that architecture can effectively work within it"

- Alexander Eisenschmidt, $2012^{103}$
An Idea of the City: a space of latent opportunity

The second on these positions understands both the city, and architecture's means to engage it, quite differently. Rather than seeing urbanisation and the city as antithetical, this position sees that the city does very much exist - but in a re-configured and ever-urbanised state, and one that often lies unrecognised. In short, the idea of the city is here understood as being emphatically, unrecognisably new - rife with latent potential and begging to be exposed as such ${ }^{104}$.

\section{Explicating Architecture's Agenda: taking a position in}

"Rather than holding back, this architecture gets involved"

- Alexander Eisenschmidt, 2012, 15

Architecture's agenda here arises through abetting the city's latent opportunities and engaging it as a space of invention, such as to reveal its unharnessed potentials. As Alexander Eisenschmidt, a key proponent of this position contends:

"the notion of the city as laboratory finds here a new meaning: no longer is it simply the territory for architectural tests, but instead it becomes the very driver of urban invention urbanisation propels urbanism" 105 
Insofar as it relates to the city by exposing those opportunities inherent in its existing state - engaging them whole-heartedly - this position may be termed an opportunistic engagement: neither resistant nor obliging, but critical nonetheless. Rather than taking a position on the city from the outside (as an indexical relationship confers), here, architecture's urban agenda is to take a position $\mathrm{in}^{106}$.

\section{Exploitative and Extrapolative Measures: using the city, to reveal the city}

Here, architecture's inevitable demand for form begins to learn from the city, reconfigures its own workings and injects itself back into the contemporary megalopolis."

- Alexander Eisenschmidt, 2012, 15

With this goal of positioning in and relating, radically, to the city in order to reveal its latency, architectural form adopts exploitative measures: 'celebrating', 'learning from' and confronting transformed urban contexts precisely on their own terms. This may mean incorporating, reworking, distilling, or exaggerating the city, in its urbanised state: through forms, shapes and visual tactics, for example. As Eisenschmidt remarks, this points towards

"an architecture of the city that is no longer against urbanisation [....] but uses [it] to productively get its way..." ${ }^{\prime 107}$

Here, reconfiguring itself strictly in relation to the city, architecture not only reflects back upon it, but moreover, diverts, directs and deflects its forces ${ }^{108}$. Notably, this opportunistic and exploitative approach dismisses formal resistance and the distinctly object-based directives above for those more directly engaged ${ }^{109}$. Simply, here architecture's urban project is drawn from a different idea of the city - as a place of opportunity and new intelligences that are, indeed, begging to be revealed and its strategies to engage are derived accordingly.

106 The agenda here is "to produce an operational architecture capable of connecting and inserting itself" (Eisenschmidt, Stranger Than Fiction: A Mission Statement 2012, 15) See also (Somol 2012)

107 (Eisenschmidt, Stranger Than Fiction: A Mission Statement 2012, $15)$.

108.

109 While these strategies are no less formally-concerned than the approach outlined above, though they may be less autonomous. They are fundamentally critical, however: resisting dominant hegemonies which oblige urbanisation and trying instead to construct and imagine new realities of , and for, the city.

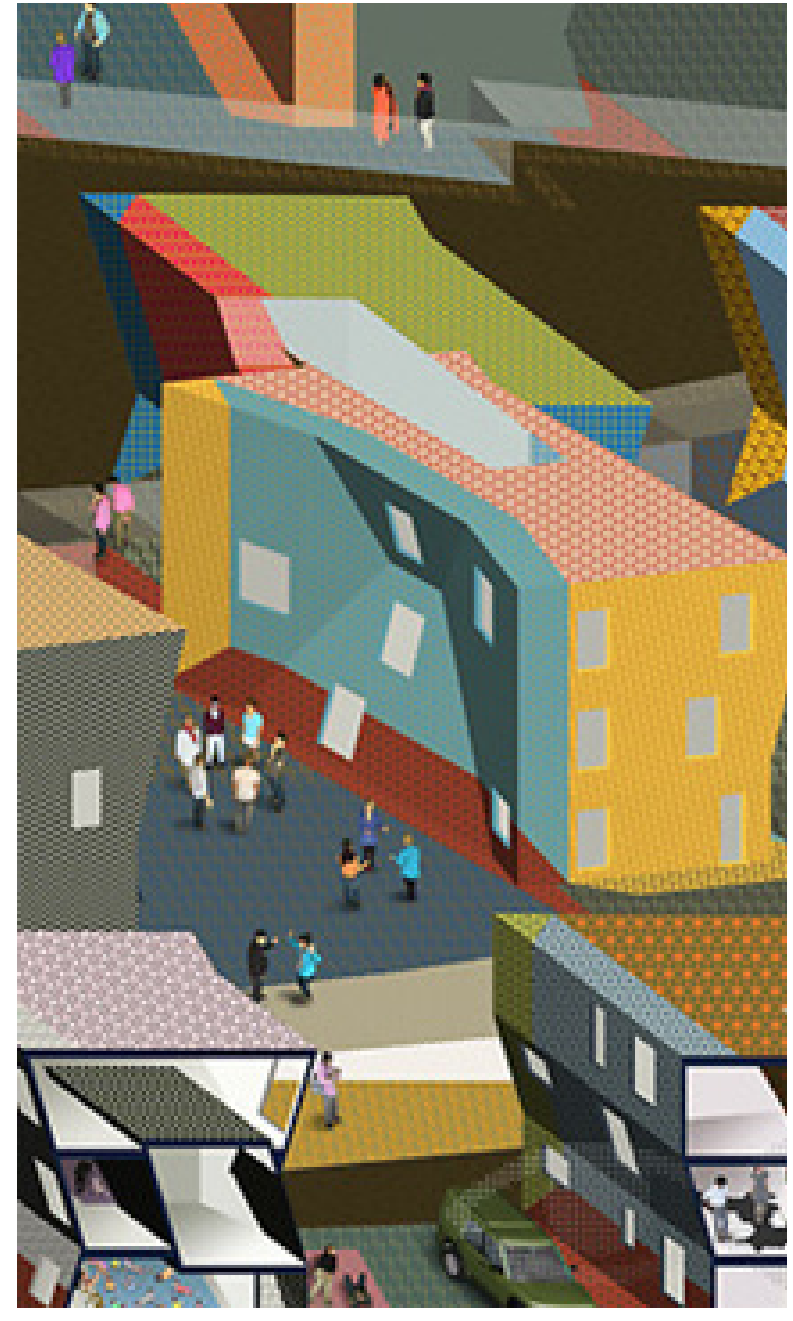

Figure 53: Zago Architecture, Property with Properties, Rialto, California (2012). The project tested a formal response to loosened boundary conditions. The concave forms of the buildings suggest a new socia / public space suggest a nuiding / public space between buildings. In this way th architectural form may be seen to activate new ideas about the city (or in this case, the suburb).

\section{Formal Strategies: relating radically to the city, in the city}

The formal operations conferred by this position seek to engage the multiple, possible conditions of new urban contexts by explicitly relating to them: a 'radical contextualism' per $\mathrm{s}^{110}$. These do not manifest as distinct formal 'strategies' ${ }^{111}$, so much as approaches that might allow urban contexts to become stimulants for critical, formal invention (and engagement). Though, these are incredibly diverse, and indeed this attitude profits 'openness' and experimentation above all as a means to achieve this agenda, here I will outline two approaches which attest to the thinking:

\section{1- Formalizing New Urban Scenarios}

As a first approach, new scenarios presented by emergent urban conditions - uncharted programmatic overlaps, social dialogues, or policy regulations, for example - are understood to present a plain opportunity for formal invention. Exploiting these scenarios as a stimulant to explore tectonics, shapes, and geometries, in an intentional, explicit and imaginative fashion, it is seen that architecture might elucidate and catalyse the city as a beacon of unharnessed opportunities - by enacting those opportunities in a forthright manner. 110 This term is used by a number of writers and practitioners in the
Architectural Design publication City Catalyst (2012).

111 As typology and even formal 'separation' might be, for example. 


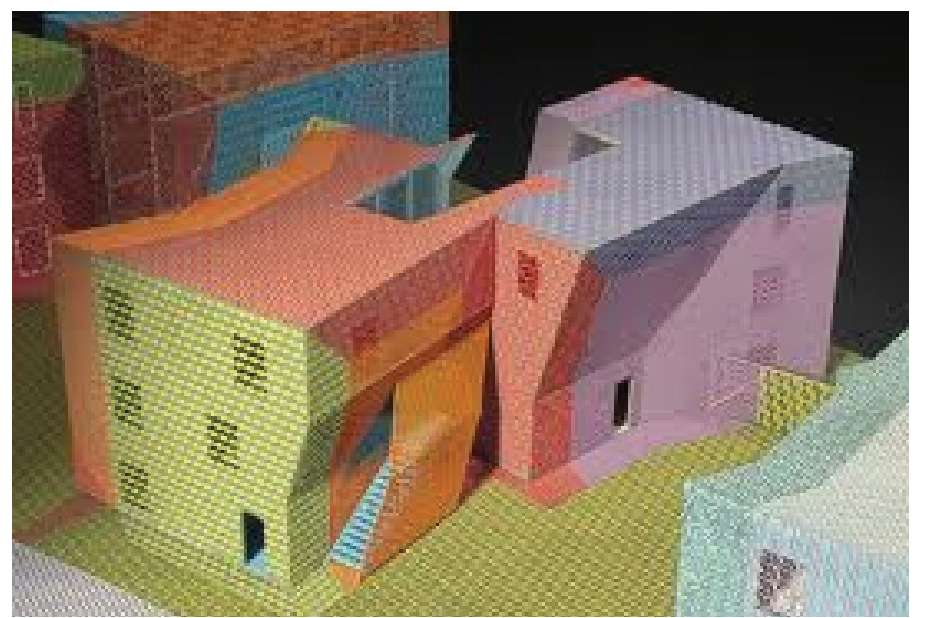

Figure 54: Exploitation of new urban opportunities is enabled through an experimental approach to an experimental approach to

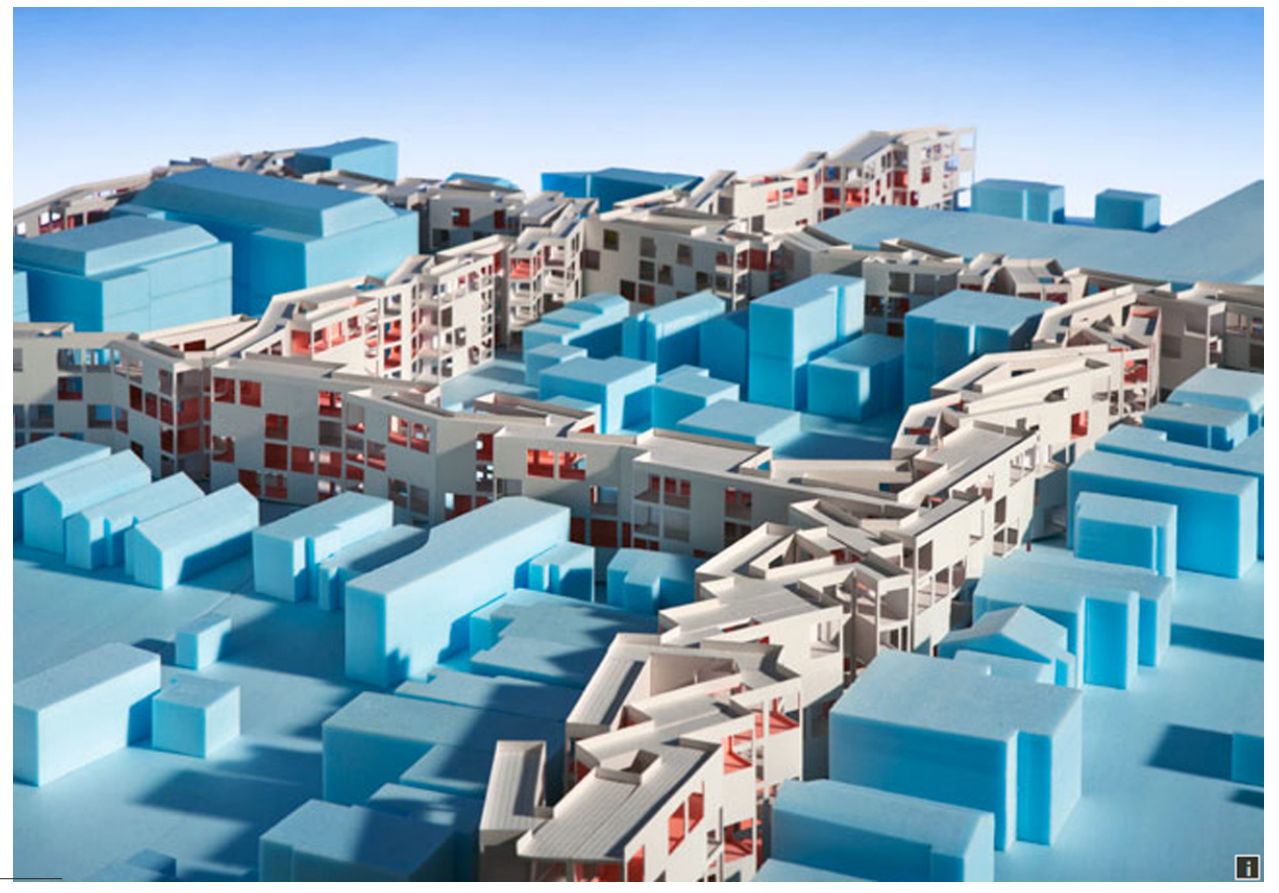

Figure 55: MOS Architects, Thoughts on a Walking City, Orange, New Jersey (2012). The project distilled the collision of living and working programmes into a potent architectural gesture activating new ideas about suburb through distinctly formal means.

Figure 56: Livework distilled as an architectural form. The project proposes a radical architectural occupation of the street - which the architects suggest has becoms sug public space, largely given over to private parking, traffic, generally dangerous for pedestrians. In this way, the building enacts and exaggerates ideas about the street as an essentially and necessarily pubic space.

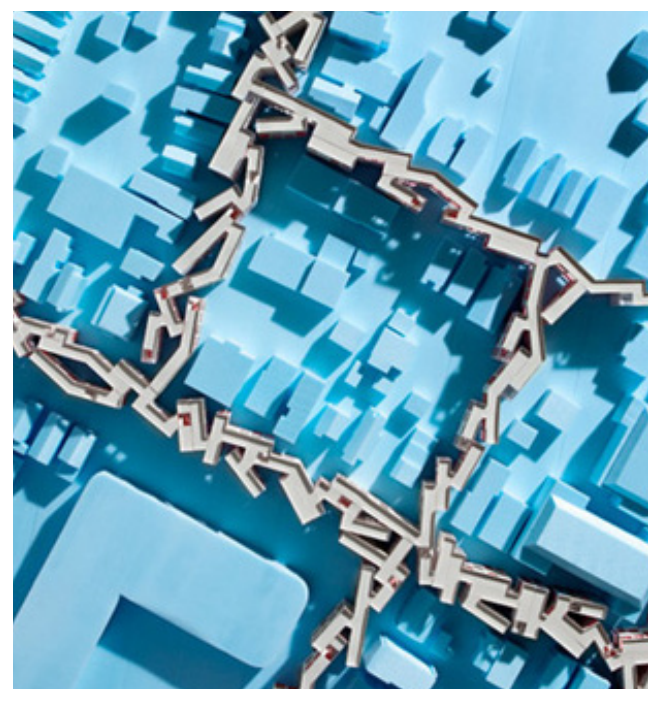

Formal experimentation is treated as a critical device to negotiate the forces of new urban contexts: not to oblige them (as the norm), but to deflect, underwrite or divert them, and in so doing, revealing hidden opportunities: spatial, programmatic, and social in nature.

12 (Eisenschmidt, Stranger Than Fiction: A Mission Statement 2012

15) uses these terms to describe how architecture might engage the city

in such a positive, open fashion (yet still be critical of its forms).

113 (Somol 2012, 110) 


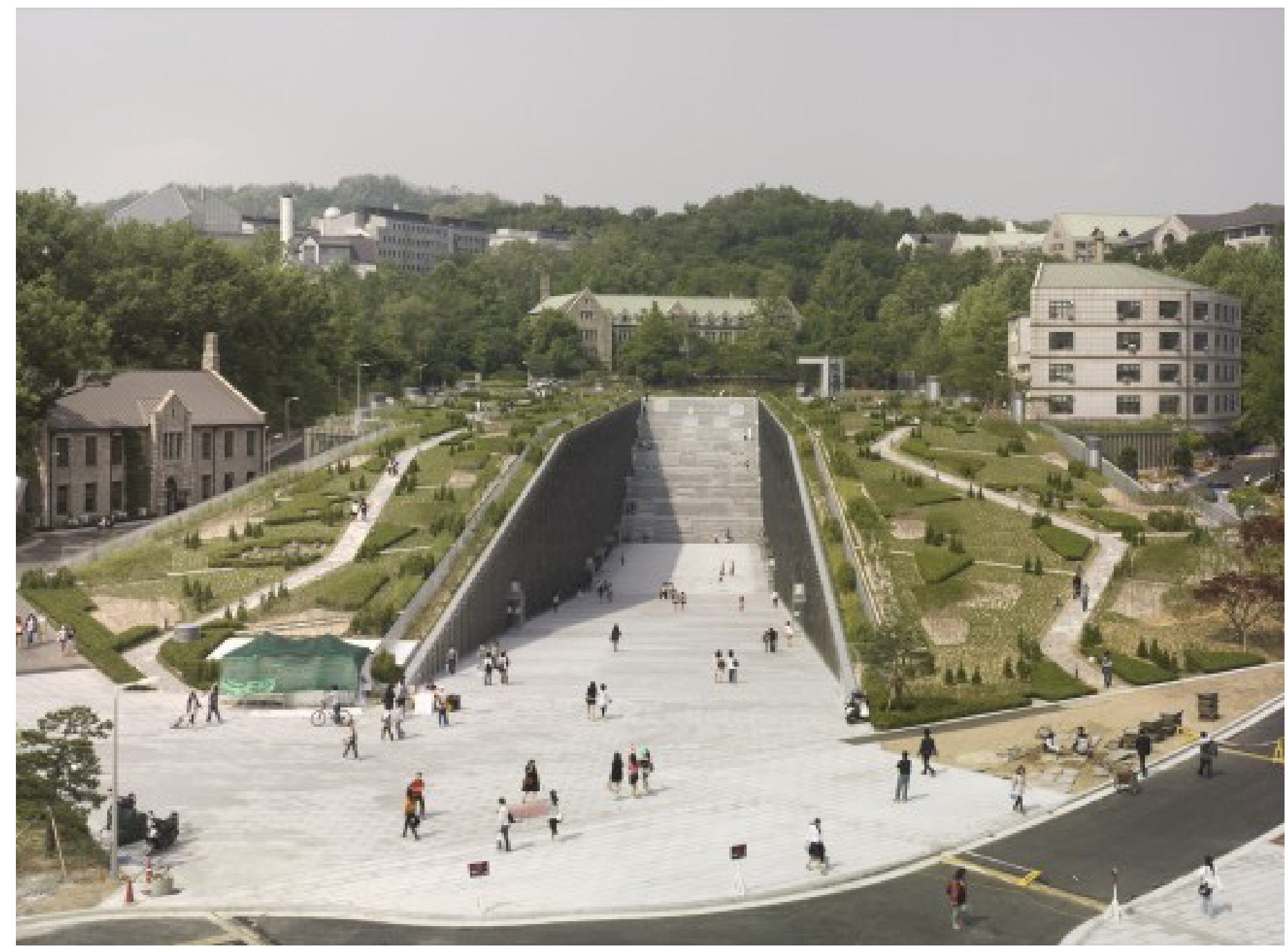

$<>$

Figures 57-60: Dominque Perrault Architecture, EWHA Women's University, Seoul, South Korea (2008). University, Seoul, South Korea (2008).
The dramatic incision into the landscape The dramatic incision into the landscap
interrogates social opportunities of this urban context, cutting a public slice out of the university: an unexpected response. The architectural form imparts upon the urban context the possibility of new relationships, and new ideas of the city. This is not a case of form following function, but rather, deflecting new urban forces as an opportunity to expose new urbanisms, and re-think architecture's means to engage the city.

\section{2- Engagement by Way of Formal In(ter)vention}

With the underlying aspiration of positioning itself in the city (indeed, through form) this approach essentially questions the limits of architectural form itself - and may see it enter into other 'territory' in order to engage. Whereas the previouslyoutlined position insists upon traditional limits of what constitutes architecture (and architectural form) - here, the emphasis on invention and engagement between the formal and the urban gives rise to challenging these limits. Taking exploitative measures, without the need to be discrete or absolute, this radical engagement might indeed manifest as physical intervention, through form. The MOS example above, for example, (fig1) cuts into what may be considered 'urban' territory - the open space of the street - not just in conceptually, but physically and formally. Occupying this territory unequivocally, the architecture imparts a new understanding to the city: not only of live-work cultures in a concentrated and inseparable dialogue, but of pedestrian dominance over the automobile.

A comparable example of this radical urban engagement through formal intervention is seen in the EWHA Women's University by Dominique Perrault. Here, architectural form is by no means resolute nor singular, but rather is radically engagedt with the urban landscape in a way that imparts (and enacts) new possibilities for public / private dialogues: exploiting the latent opportunities of a university and an open, public space in close proximity (figures 57-60).
Not dissimilarly, the Oslo Opera House by Snøhetta formalizes public and social dynamics latent within an urban context, by seamlessly blurring the architectural object-form, public ground space and urban landscape together as a singular formal gesture. Again, here architectural form is not separate, nor absolute: but is rather directly and radically deployed as a means to exploit new urban opportunities - and, indeed enact them. (figure 61).

In these examples, architecture firmly positions itself in its context through an approach to form which, while diverging far from the resolute, singular or separate predilections of the first position, need be no less potent or intentional.

\begin{abstract}
Whereas the previously-outlined position insists upon traditional limits of what constitutes architecture (and architectural form) - here, the emphasis on invention and engagement between the formal and the urban gives rise to challenging these limits.
\end{abstract}

\section{In Summation}

With the objective to engage and catalyse the city by revealing its latent opportunities, these approaches enable architecture firstly, to exploit the urban conditions (social, spatial, programmatic) as a means of formal invention; and secondly, to position itself in the city, such that it may enact these new opportunities tout court. As opposed to the formal-specificity necessitated by the previous 'resistant' approach this position lends itself to a more liberal approach to form, but one that is no less focused on the city as its project. 

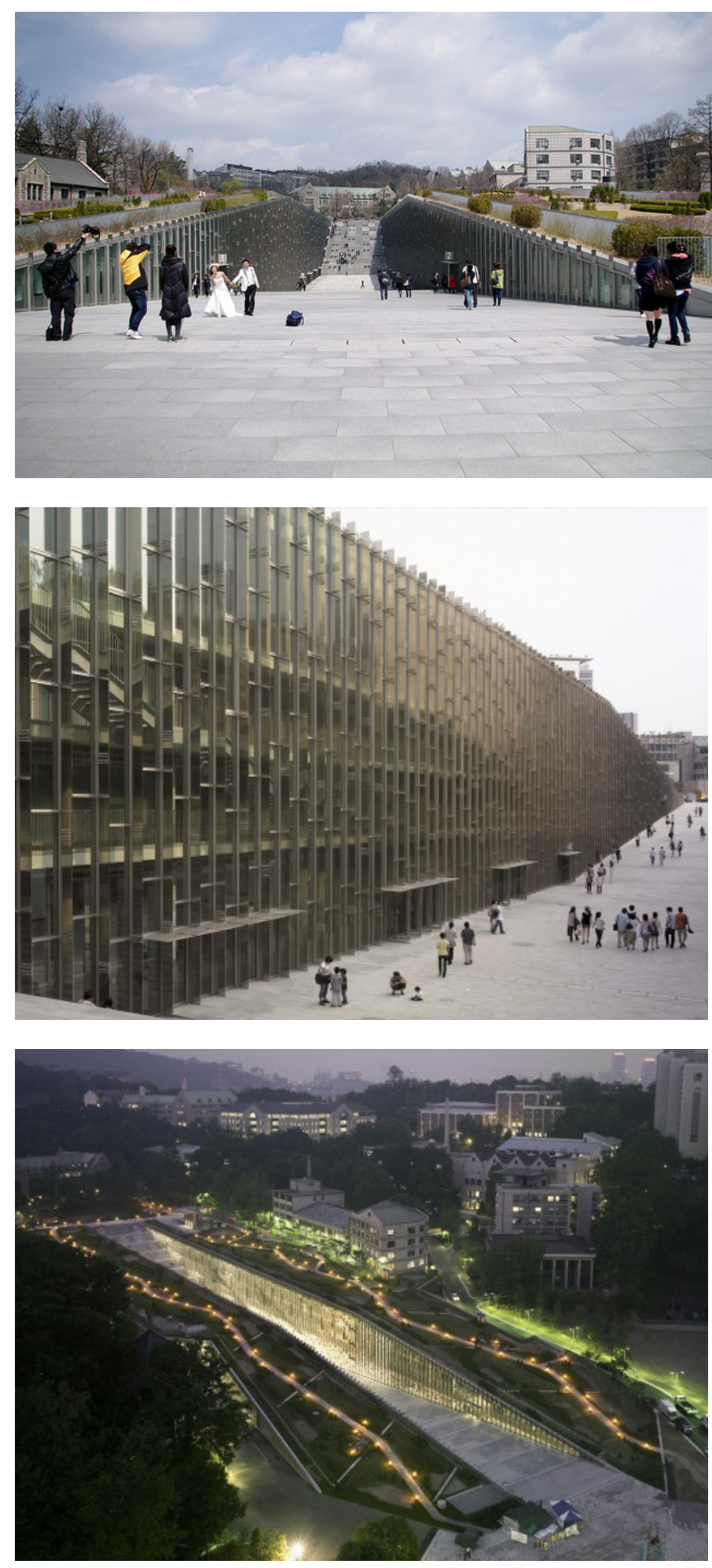

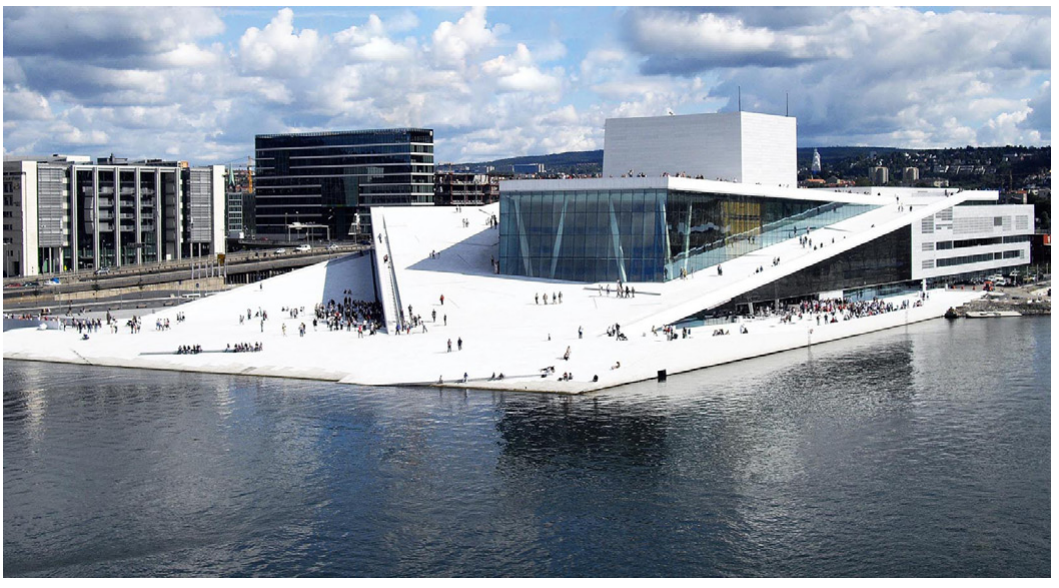

Figure 61: Oslo Opera House, Snøhetta, Oslo, Norway (2007). The manipulation of architectural form and its integration with the exterior environment sees the roof of the opera house to becomes a public plaza hat wraps up and around as a coninuous gesture. As these different spaces and programs fold into one another, new public /social dialogues are actively facilitated by the architectural form. In this way, the architecture engages the city as a space of latent opportunity, and by positioning itself firmly within it, reveals it as such.
Figure 62. A summary of the two ideas discussed: compared their understandings of the city, their agenda for architecture, and the formal operations put forward by each.

\begin{tabular}{|c|c|c|}
\hline & $\begin{array}{l}\text { Terms of Engagement 1: } \\
\text { Critical Formal Resistance }\end{array}$ & $\begin{array}{l}\text { Terms of Engagement 2: } \\
\text { Critical Formal Opportunism }\end{array}$ \\
\hline $\begin{array}{l}\text { IDEA OF THE CITY } \\
\text { (Architecture's project) }\end{array}$ & $\begin{array}{l}\text { Urban Archipelego } \\
\text { (city as political space) }\end{array}$ & $\begin{array}{l}\text { City as reconfigured } \\
\text { by urbanisation: an } \\
\text { unrecognisable state and a } \\
\text { space of opportunity. }\end{array}$ \\
\hline $\begin{array}{l}\text { ARCHITECTURE'S } \\
\text { AGENDA }\end{array}$ & $\begin{array}{l}\text { To reinstate the archipelago } \\
\text { (and resist urbanisation) }\end{array}$ & $\begin{array}{l}\text { To reveal the city in its new } \\
\text { state, and as a space of } \\
\text { opportunites }\end{array}$ \\
\hline $\begin{array}{l}\text { ARCHITECTURE'S } \\
\text { MEANS TO ENGAGE } \\
\text { (Through form) }\end{array}$ & $\begin{array}{l}\text { To 'take a position on' } \\
\text { through an indexical } \\
\text { relationship to the city, } \\
\text { and integration (through } \\
\text { separation) } \\
\text { To take a position on! }\end{array}$ & $\begin{array}{l}\text { To 'take a position in' } \\
\text { through exploitative } \\
\text { measures and a radical, overt, } \\
\text { exagerrated relationship to the } \\
\text { urban context } \\
\text { (to insert itself in!) }\end{array}$ \\
\hline $\begin{array}{l}\text { FORMAL } \\
\text { OPERATIONS }\end{array}$ & $\begin{array}{l}\text { Seperation } \\
\text { Composition } \\
\text { Resoluteness } \\
\text { Typology }\end{array}$ & $\begin{array}{l}\text { Formalization of social events } \\
\text { Formalization of opportunities } \\
\text { Exaggeration } \\
\text { Radical contextualism }\end{array}$ \\
\hline
\end{tabular}




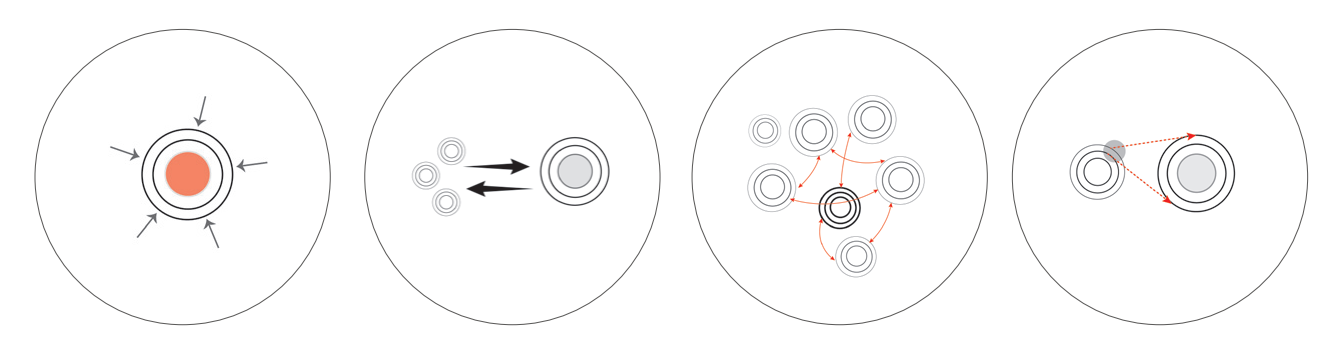

\subsection{Speaking of the Sub-Centre: negotiating a position between on and in}

The two approaches discussed here - each addressing a renewed attentiveness to architectural form as means to engage differing understandings of the city - offer a compelling way forth for the sub-centre as an distinct urban idea: one arising, in fact, quite on account of their disparity.

Essentially, the fundamental point of difference between these two positions arises in their understandings of the city, and the terms of engagement which they accordingly derive: one seeing the city as an inherently public, political space in need of reinstatement; the other seeing it as a beacon of opportunities in need of abetment. Yet, in relation to a sub-centre context, each of these ideas of the city appears to have an indisputable resonance.

This is simply explained. Firstly: inasmuch as the sub-centre may be construed as a holistic alternative to the city-centre - a veritable state of urbanity that is absolutely social, political, cultural, and public in nature - the idea of the city embodied by the first position (and attesting absolutely to these fundamentally 'city' qualities') is quite clearly resonant. Moreover, its underlying understanding that the prevalence of economic imperatives may have a detrimental effect on these essential political and public qualities has an indisputable bearing on the sub-centre - indeed, given the tendency for these aspects to unduly characterise it, as such. Certainly, in an effort to instil ideas of gravity, vitality, and centrality to the subcentre; the former approach seems particularly pertinent.

Yet, the sub-centre as an emergent urban space necessary transcends these fundamental and more traditional 'city' concepts, too: on account of its rapid emergence; its forged urbanity; the inherent paradoxes that plague it; and its critical role as part of a larger urban system - not as the centre but one of many centres. With these opportunities at stake, the latter position - which subscribes to an understanding of the city that is rife with uncharted potential - appears to have a plain bearing on the sub-centre as an idea. Here, the unique opportunities of the sub-centre as a space that is not a citycentre per se, but potentially something quite distinct (and with polyvalent possibilities) might be addressed.

\begin{abstract}
...the fundamental point of difference between these two positions arises in their understandings of the city [...] one seeing [it] as an inherently public, political space in need of reinstatement; the other seeing it as a beacon of opportunities in need of abetment. Yet, in relation to a sub-centre context, each of these ideas of the city appears to have an indisputable resonance.
\end{abstract}

On this simple account, the conciliation of these two differing positions -whether in dialogue, or juxtaposition, or negotiation - may present some fertile opportunities for architecture to engage the sub-centre as an urban context that is ultimately (or ideally) characterised both by qualities of political, cultural and public gravity on the one hand, and latent, uncharted urban opportunity, on the other.

From here, the obvious - and only - way forward is to consider how their terms of engagement might be effectively reconciled: the first seeking to take a position on through formal resistance, the second, to take a position in through formal engagement. So, what are the actual possibilities of bringing the operative strategies offered by each - one of resistance, resoluteness, and formal specificity; the other of exploitation, extrapolation and formal intervention - in dialogue? How can the former's penchant for autonomous, separate forms and the second's for radical formal contextual invention be reconciled and re-appropriated in a sub-centre context? How might the seriousness of the former be juxtaposed with the often-playful liberty of the latter? Negotiating these two approaches critically and carefully, in a dialectical synthesis - rather than crudely assimilating them - a number of fertile modes for architecture to engage the sub-centre as its project may be afforded. 


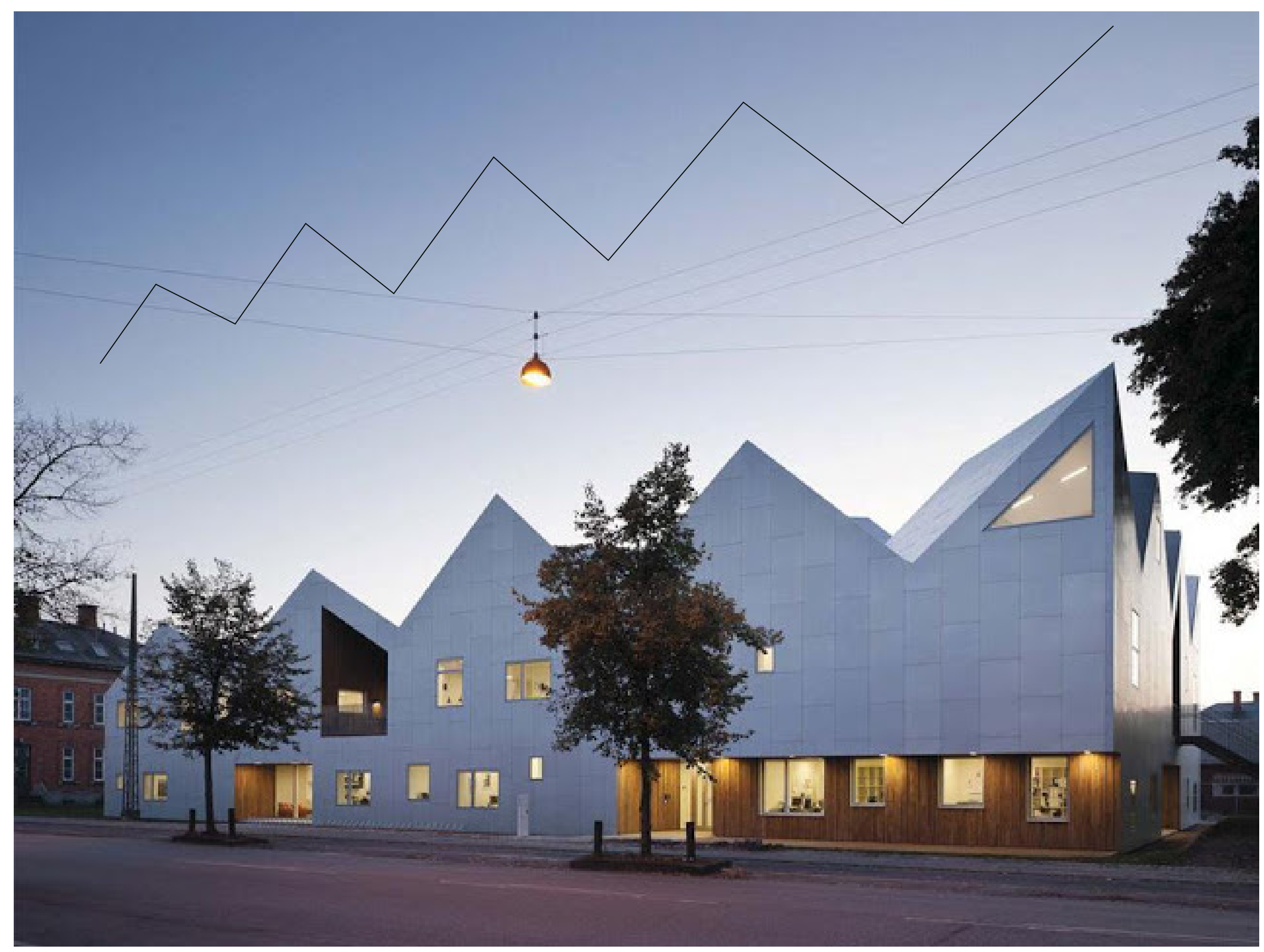

\section{Making Room for the (in)Formal: resolute yet} radical

Firstly, synthesising an insistence on formal specificity on the one hand, and radically-contextual formal derivations on the other, amounts to a number of compelling possibilities. Immediately, this invites the active negotiation of directives for separate, confrontational Form, and form intentionally and expressly derived from the often-chaotic opportunities of transforming urbanisms (indeed, often lending itself to informal outputs). This opportunity is particularly striking given the multiple transitions embodied in the emergent subcentre, whereby urban functions frequently and rapidly collide with residential, for example.

Here, juxtaposing separate resolute object-form with a permeable fertile building edge that enacts new social dialogues; or considering how the agonistic countercomposed parts of a building might be brought into dialogue with a formal enactment of public-private relations, may offer vast possibilities to attest to a political, public space that is inherently different from the city. In these cases, the imperative is not to abruptly amalgamate the two dictates - but to hold them up against one another, testing: contrasting, synthesising as fit - in a critical fashion; and with the sub-centre as a project absolutely in mind. (figure $64+65$, and $66-7$, overleaf)

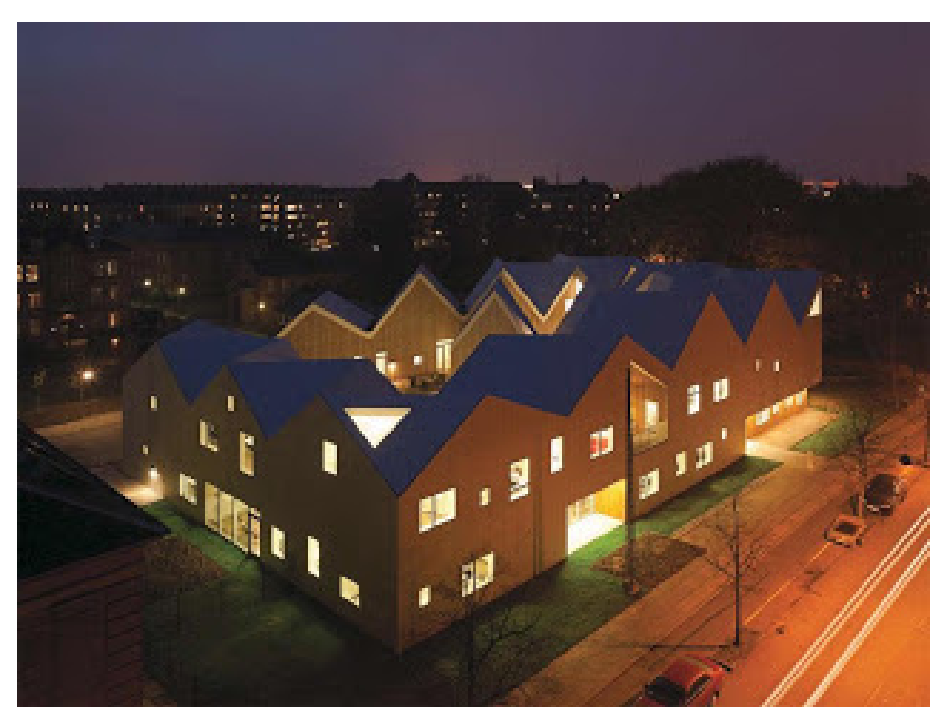

Figures 64 +65: Nord Architects, Healthcare Centre For Cancer Patients, Copenhagen, Denmark (2010). Located in an urbanizing suburb of Copenhagen, this architecture is a clear object-form, negotiating typology as a means to relate to the context (a series of gables and perimeter block); and presenting an individual form whose envelope is clearly articulated and possesses a certain separateness. Yet, with the subtle informality of roof line, and the incisive puncturing of the form to invert its interior - revealing it to the street - the opportunities presented by a hospital in a residential setting are exploited. A public-private barrier is negotiated and new dialogues actively invited through the architecture's form and surface articulation. In this way, the building sits between these two approaches to engage the city: a formally- resolute and resistant object; and an endearing, explicit formal manipulation of the opportunities latent in transforming urban contexts. 

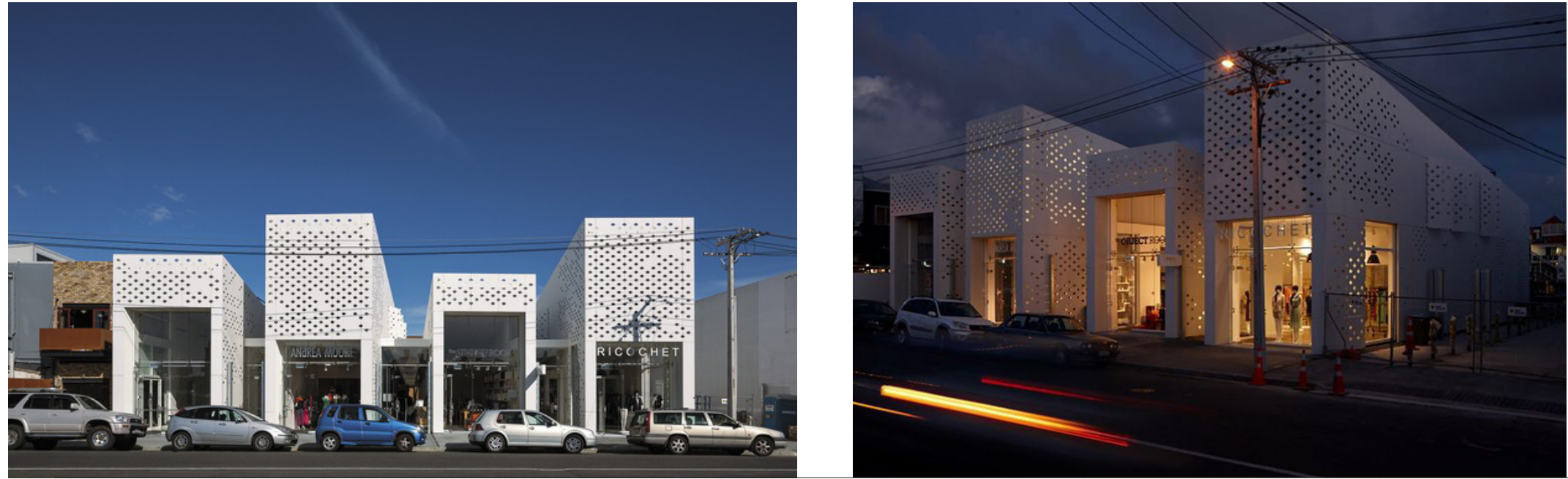

Figure 66+ 67: A Resolute Informal: RTA Studio, Retail Precinct, 2012. This design presents resolute, separate form(s); carefully composed in a part-to-whole relationship. Yet the scale and informality of these different parts allows the building to engage anurthe building to engage an urbanizing suburb as a context that is neither city nor suburb but somewhere between. In this way, the architectural forms induces a certain urbanity, but a moderated one.

\section{Stretching the 'limits' of Architectural Form (but asserting them, no less)}

\section{Separate yet engaged / object yet intervention}

As a second possibility, negotiating these two positions renders the notion of the architectural 'limit' a fertile ground for discussion. Whereas the former insists upon a more traditional understanding of architectural 'form' (as object form), the latter, inherently seeks new ideas about what constitutes architectural 'form' in these contexts (and, fundamentally, the need for this to be explored). In this way, for the former the building's edge constitutes architecture's primary ability to be political and therefore must be consciously articulated as a 'limit'; for the latter it is often an fertile place for enacting new social dialogues ${ }^{114}$. Likewise, where the first stresses the potency of the object-form, the second, with its penchant for formal engagement, often insists that it shifts into other territory.

In dialogue these seem to summon an approach which articulates the limits and separateness of the architectural form (indeed, as if it were an object) yet may also allow it to intervene on urban contexts and sites in an exploitative and engaged manner. This has implications both for the 'limits' of architecture (physically), and the edge of what constitutes architecture (and architectural form) in a more general sense (figure. 68).

Figure 68: Peter Eisenman Architects, City of Culture, Galicia, Spain (2011). On the one hand, the architectural form articulates its edge and 'limits', and allows each element to become distinct and separate (as well as the space between them to be other, it is formally engaged and fundamentally intervening on the landscape, crossing into other 'territory' beyond an understanding of object form. ...for the former the building's edge constitutes architecture's primary ability to be political and therefore must be consciously articulated as a 'limit'; for the latter it is often an fertile place for enacting new social dialogues

114 As seen in the Zago Architecture project, above, for example. 


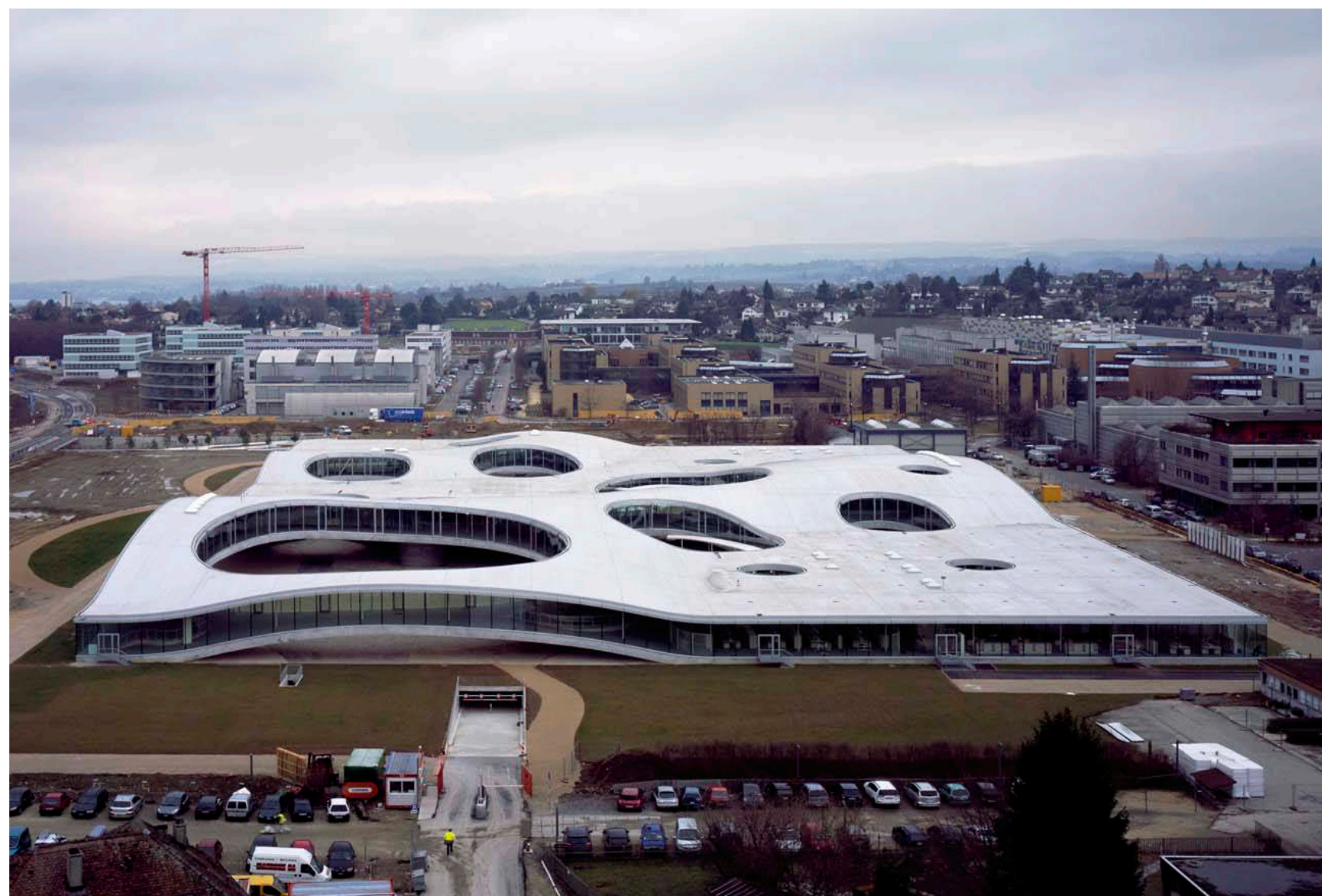

\section{Exploitation of Typology: integration though} invention.

Numerous opportunities arise around the possibility of bringing typology (as a strategy to relate to the city as a whole through a point of consistency) into dialogue with a more inventive, exploratory approach to architectural form - as an effort to distil social dialogues, for example. Indeed, typology has often been used as a platform for a contextual, formal innovation, in a way that does not seem dissimilar to this, in recent years. ${ }^{115}$

Notably, when deploying typology in an emerging sub-centre context, it seems likely that strictly'urban'116 typologies may not always be appropriate. Here, in order to engage the transition inherent in the emergent sub-centre, there may be scope to address sub-urban or peripheral urban typologies (whether it be terraces or even semi-detached housing) in a comparable manner: that is, as a means of site specificity and a point of continuity, that may allow formal differentiation within the wider setting. (figure 69).

15 See, for example Lee and Jacoby (2011)

116 That is, for example, the plinth as discussed earlier; tower blocks; or higher-density typologies common to urbanized contexts but not necessary peripheral locations.
Numerous opportunities arise around the possibility of bringing typology (as a strategy to relate to the city as a whole through a point of consistency) into dialogue with a more inventive, exploratory approach to architectural form.

\section{Figure 69: SAANA, Rolex Learning Centre, Lausanne, Switzerland (2009). This project deploys the Matt building typology as a way of asserting itself as a separate object within its context, and defining the space around it with its square geometry in plan (enabling a clearly expressed building edge). Yet, with voids clearly puncturing through the form, public spaces more typically confined to the exterior are brought into the building, sponsoring new programmatic dialogues and overlaps.}

Moreover, with its fluid undulating ground plane, the architectural form tests the interior space (a university library) as a continuation of the urban landscape - a highly imaginative approach which attests to new urban possibilities, by formalizing them. 


\section{Composing Densities: toward an agonistic opportunism}

As a final possibility (though there are many more not to be discussed here) the effective synthesis of these approaches, to engage the emergent sub-centre, present opportunities to bring together an emphasis on carefully articulated part-towhole composition with the exploitation of higher densities as a formal opportunity. This might suggest, for example, the composition of parts of the building in a way that they may both embody notions of difference, conflict and agonism, yet allow new social dialogues and programmatic overlaps to be enacted (and formalized). This seems particularly relevant given the likely influx of medium and higher density housing to these densifying environments. (figure. 70).

\section{In-forming a conclusion}

\section{Radical and Resolute: an architecture absolutely engaged?}

Despite their differing predilections (and predications) the two positions discussed here need not be irreconcilable. Rather, their juxtaposition affords a particularly fertile ground for formal exploration, and one that resounds with the subcentre par excellence - as an urban idea characterised equally by centrality and urbanity as opportunity, differentiation and paradox. Though the opportunities have only been outlined briefly, it seems that collapsing the critical, resistant and absolute directives of one approach with the radical, opportunistic engagement of the other, may indeed offer an effective means for architecture to absolutely-engage the subcentre as its project.

Of course there are numerous complications which arise in relation to these primarily formal architectural 'findings', and which are as general and specific as the findings themselves. Firstly, the underlying endeavor here for architecture to be both critical and engaged (or indeed, critically-engaged) belies a familiar, broader dilemma for the discipline: negotiating between architecture as critical mass on the one hand, and a cultural-social instrument on the other ${ }^{117}$. Indeed, theory and practice alike have perpetually struggled between these two agendas for (or understandings of) architecture - and

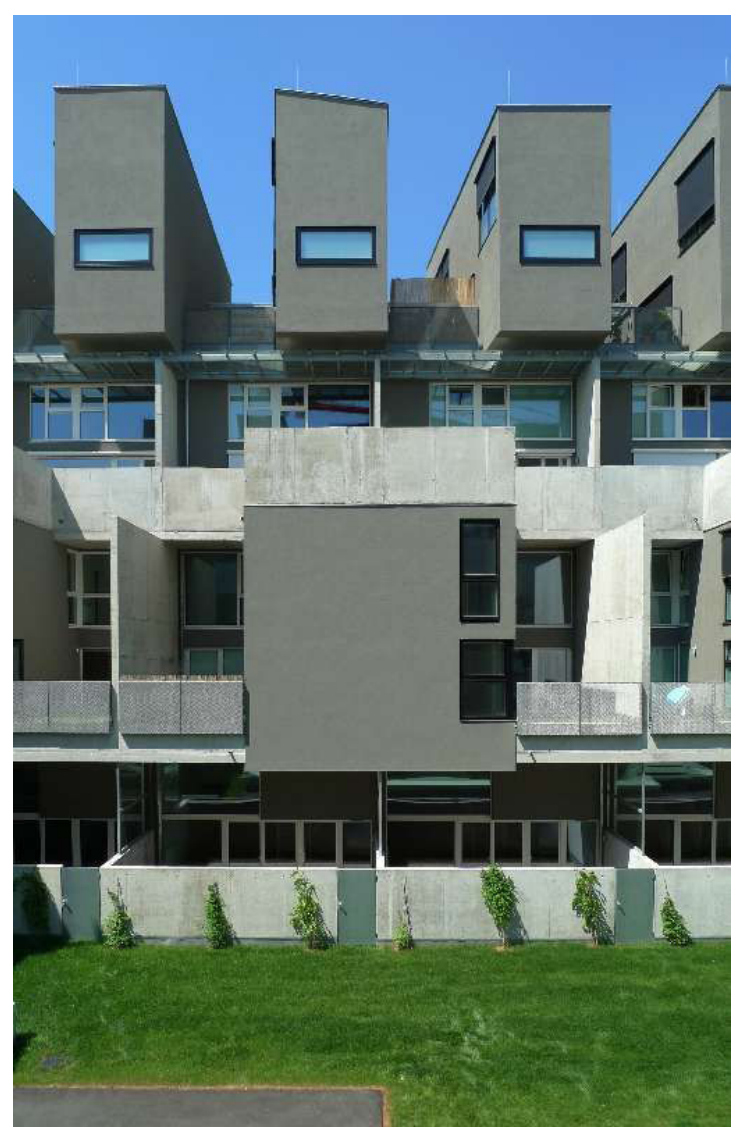

Figure 70: ARTEC Architects with Neues Lebel housing association, Die Bremer Stadtmusikanten, Vienna, 2009. This housing project articulates its sep. This housing project articulate elements in a resolute fashion, allowing a certain tension to arise between them compositionally. Yet, there is also a dynamic and even 'informal' manner to the arranagement of forms, which may be seen to deliver ideas about social dialogues.

continues to do so - with ever-polarising results. Withou claiming to resolve this tension by any means, this approach somewhat circumvents it: firstly, by limiting architecture's instrumental 'agenda' to the sub-centre (as an urban idea), and secondly, by negotiating the possibility of this instrumentality directly and explicitly through architectural form. In this way, offering a formal strategy whereby architecture might take a position in (engage) and on (critique) a distinct urban context this approach essentially upholds an architecture as a critical urban instrument.

The effective'contingency'of other concerns in this way presents a second - and more pragmatic - complication in relation to this argument. There are, of course, inherent difficulties in letting these 'synthesised' terms of engagement be played out effectively in practice, given that their overarching focus on architectural form is inevitably to be confounded by further concerns that will invariably arise in a real context. The subcentre (as any urban condition) is in practice, rife with nuanced social and cultural anomalies, policies, regulations, political constructs, and distinct physical and spatial conditions - all of which are likely to inflect a resistant-opportunistic formal agenda for architecture therein. To this end, this strategic approach to engage the sub-centre must be brought into careful, critical dialogue with these concerns if it is to be effectual: a matter which can only truly be tested against actual design contexts (and will be discussed in the exegesis, presently). 
...[the juxtaposition of these two approaches] affords a particularly fertile ground for formal exploration, and one that resounds with the sub-centre [...] as an urban idea characterised equally by centrality and urbanity as opportunity, differentiation and paradox.

\section{On a Problem and an Opportunity}

Still, ultimately this formal approach - and the opportunity it may present for architecture - should not be assessed on its own accord per se, but must be held up precisely against the problem that propelled it. This is a broad urban phenomenon whose current image, objectives and generative strategies seem to afford it little chance to liberate itself from the economic and functional motivations guiding its emergence; and moreover, a limited means to evolve as a veritable centre and an opportunity-ridden space within the polycentric region it is quickly coming to characterize. To this end, it is one that appears to be desperately seeking architecture's renewed, critical attention. Taking this urban provocation seriously, this radical-resolute formal approach may allow architecture in this context to do what both the shopping mall (in its complacency) and contemporary fluid forms (in its denial) broadly fail to: attest to an idea of the sub-centre that is indeed paradoxical, transitional; central, urban, political and essentially inextricable from a larger, multi-centered urban structure.

In this capacity, architecture may operate as sub-centre medium - instrumental on this urban context by actively taking a position on it, within it. As such, it may serve to catalyze the emergence of the sub-centre as an holistic urban place in the polycentric city - and, in so doing, afford architecture a revised agenda therein.

Fundamentally then, the urban problem and architectural opportunity at stake here cannot be separated - but must be understood as plainly and necessarily inextricable. Only by considering them as such, and probing questions for each accordingly, can architecture and the city (in its ever-varied manifestations of which the sub-centre is but one) hope to become productively, actively and effectively engaged - and, can architecture expect to address the city as its essential project.

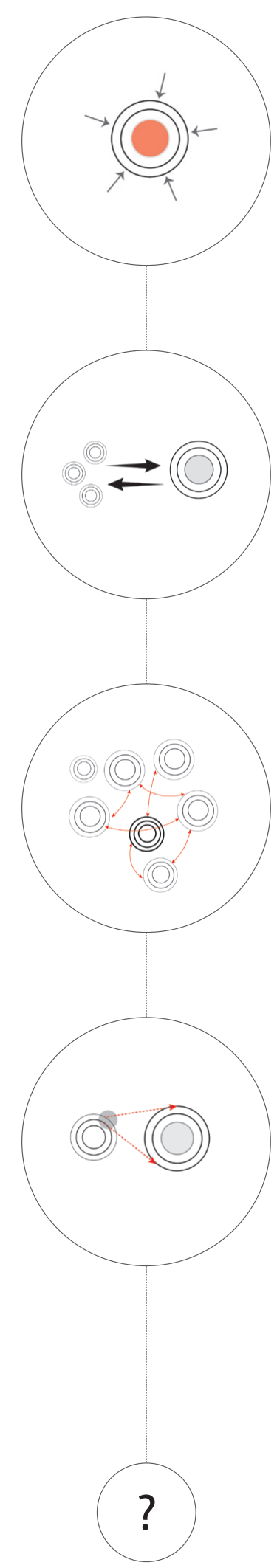

Figure 71: The Sub-centre as Architecture's Project. Architecture might engage the sub-centre as its project, through a radical, resolute approach to architectural form. 


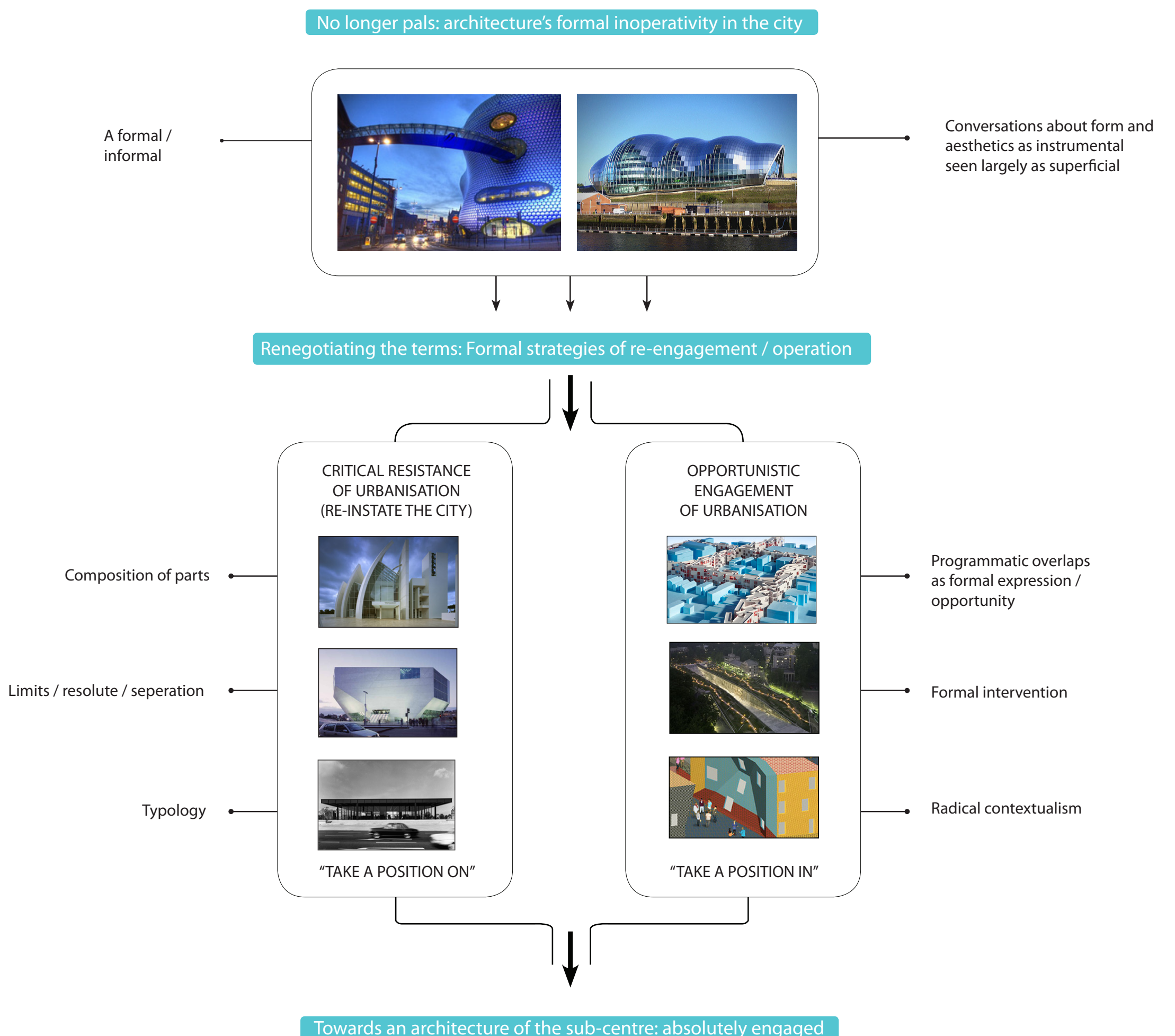

Towards an architecture of the sub-centre: absolutely engaged
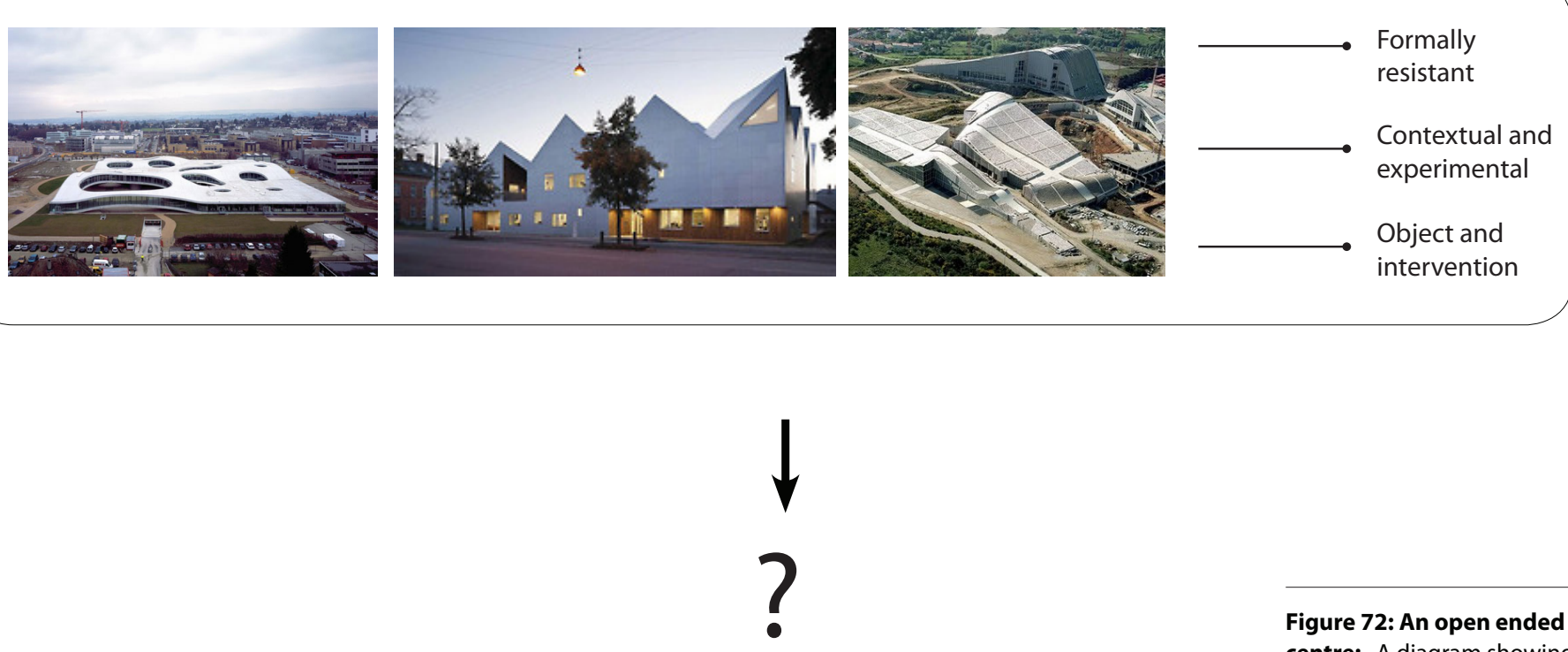

Figure 72: An open ended conclusion for the subcentre: A diagram showing the basic argument underlying this chapter. 


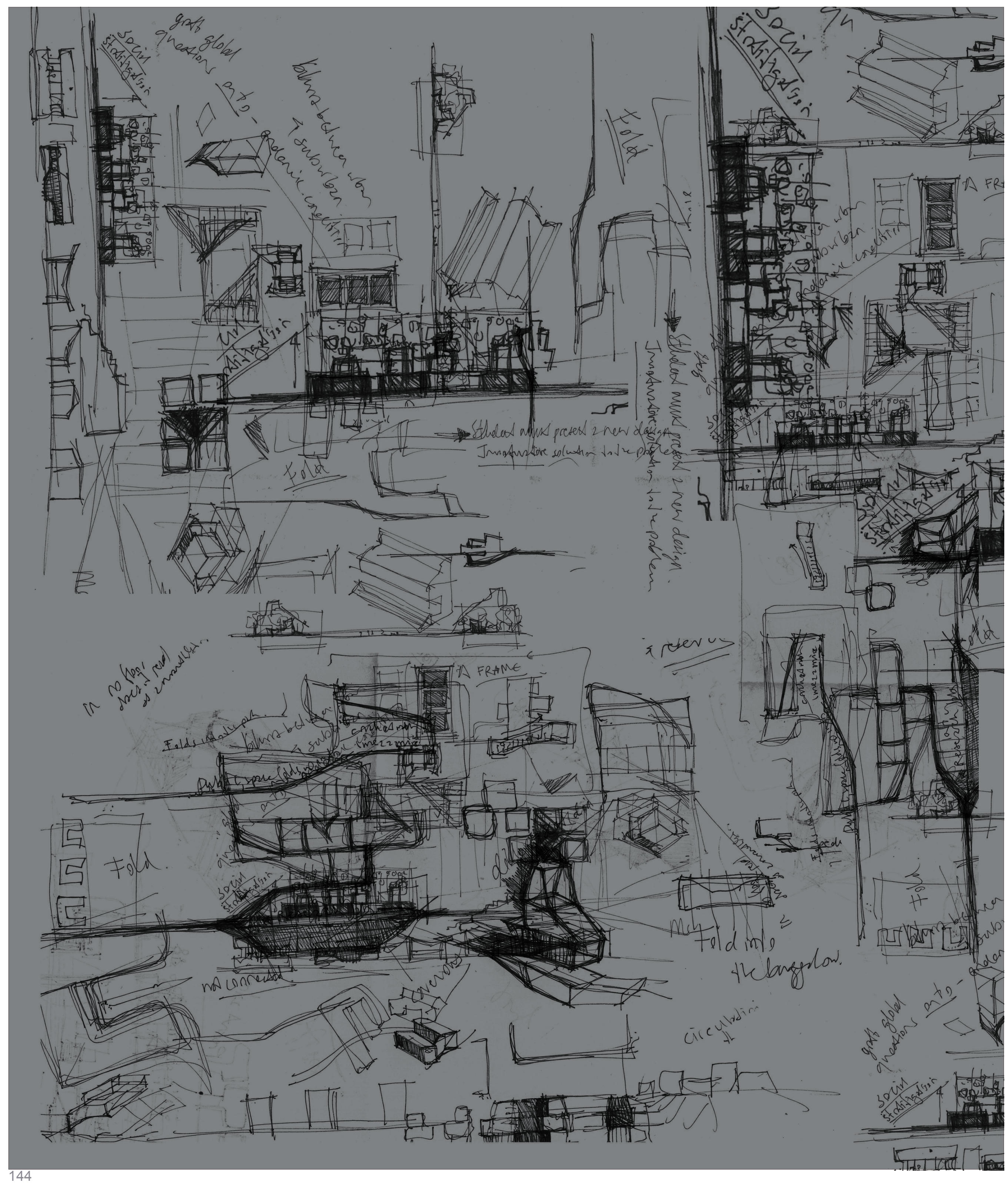


舟

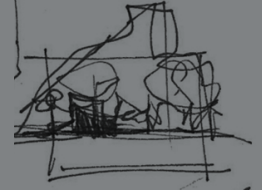

IA FRTME

$\sqrt{\frac{10}{5}}$

F 1

$\Rightarrow$ INA

tor

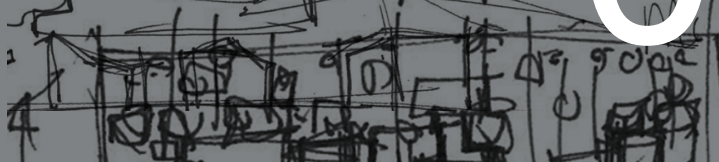

4 (1)

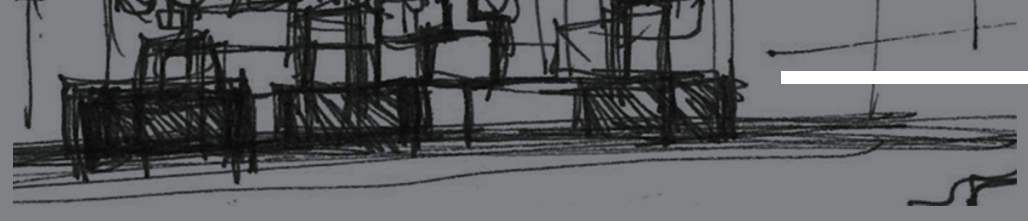

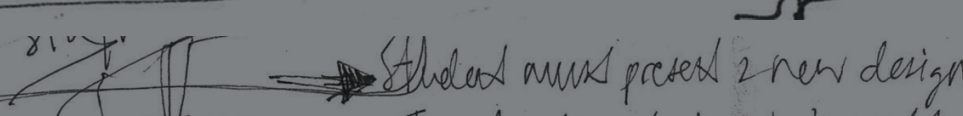

L Reflection ongal

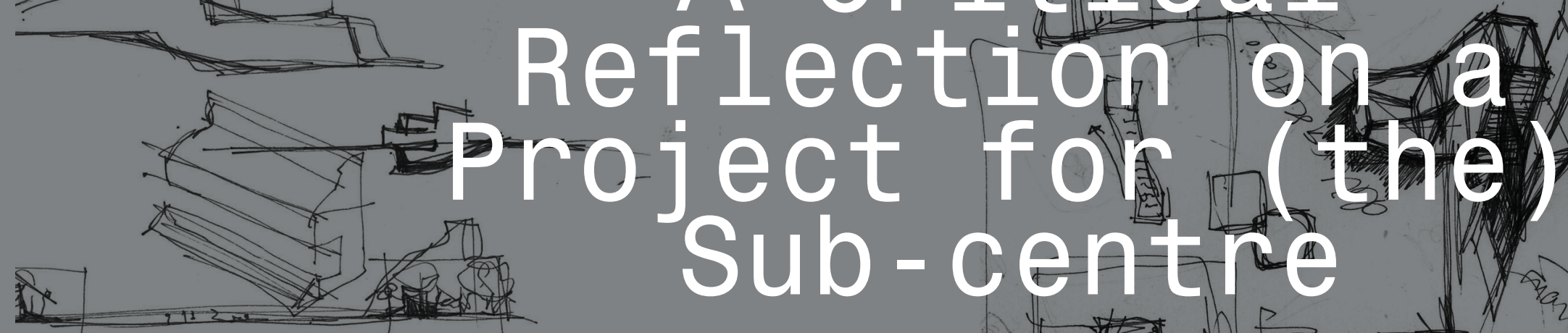

(nin o o

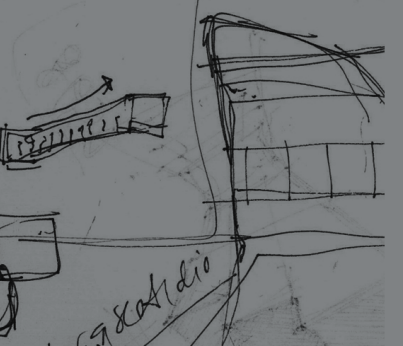

If

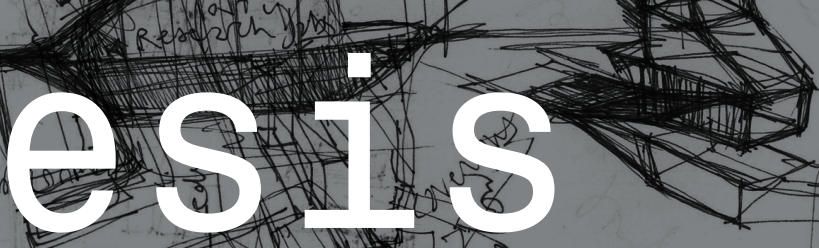

4

$\cos _{2} \sin ^{2}$

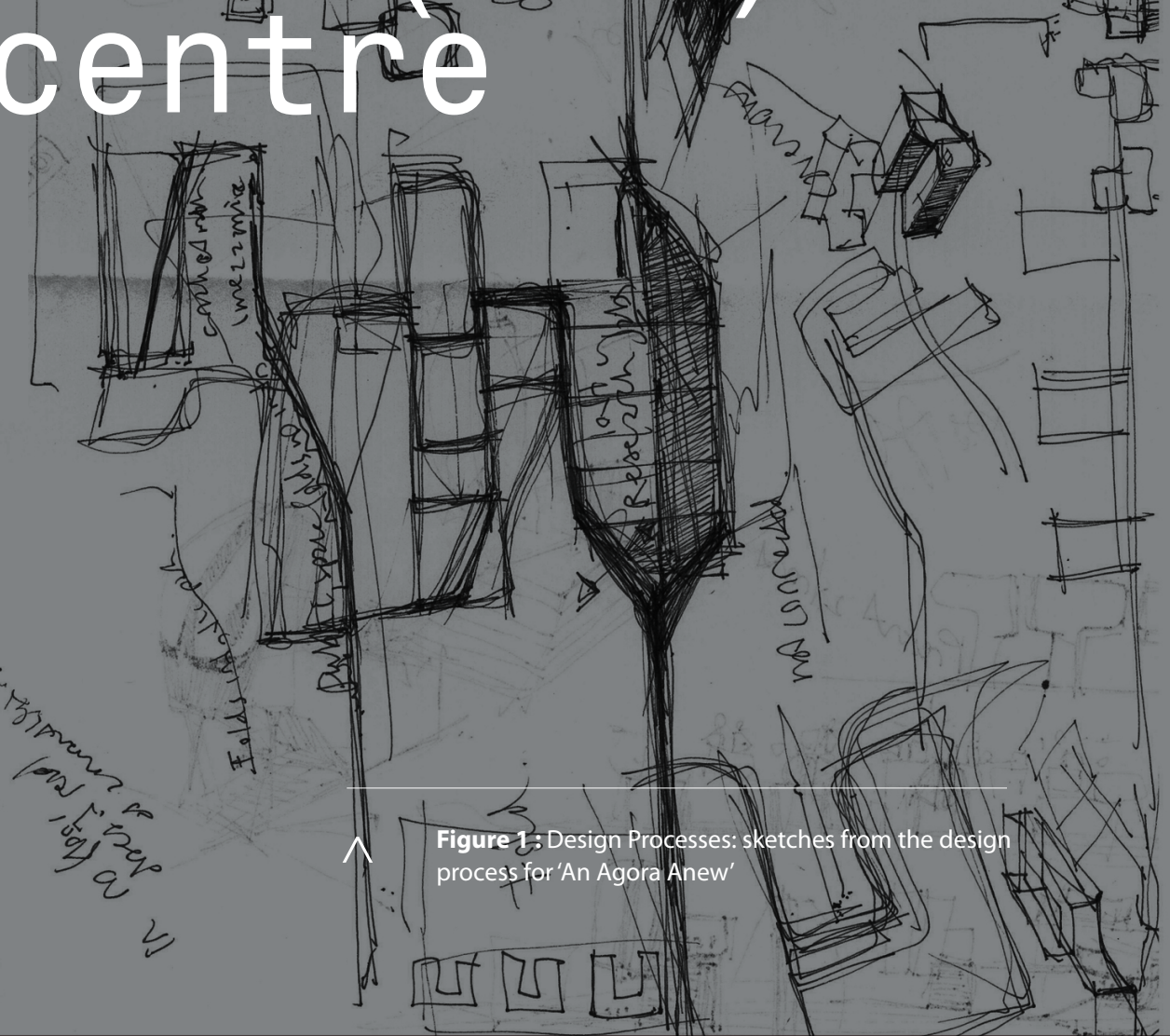

4 पाए।

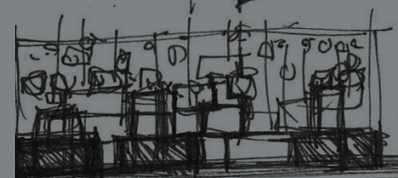

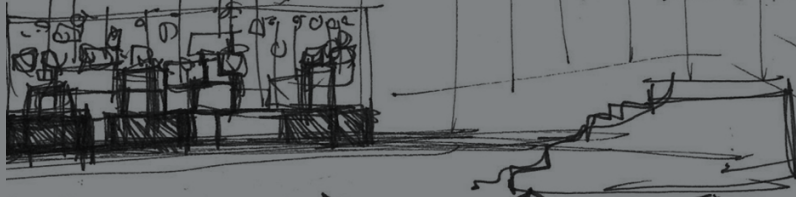

and as 


\section{In-forming: reflecting upon strategies towards a sub-centre architecture}

This section is a visual and written discussion of the design project - An Agora Anew - as a project for the sub-centre. This is enabled both in light of the argument put forward in the previous chapter, and the underlying objective of the design to explore an architectural response to an emergent sub-centre context. Notably, the intention here is not a postrationalization, self-affirmation, nor even an attempt to expose its limitations per se. Rather, it is meant as a critical and informed understanding of the project (somewhat objectively).

Initially, a series of formal strategies deployed throughout the design process are briefly discussed - organized not chronologically, but in terms of their salient bearing on the final outcome. Notably, these strategies were not preconceived or premeditated - but rather evolved with the design project itself (as well as in relation to the broader research, most probably, though not explicitly). Given the absence of program or other 'constraints' in the early stages of the design, however, these formal strategies played as significant a role in the final output as anything else - and to this end, afford an understanding of what was done. (see figure 2)

Identifying these strategies then gives way to a critical interpretation of the design: firstly, through four ways in which it may been seen to engage its sub-centre context, and then through a broader discussion of An Agora Anew (holistically) as a project for the sub-centre. 


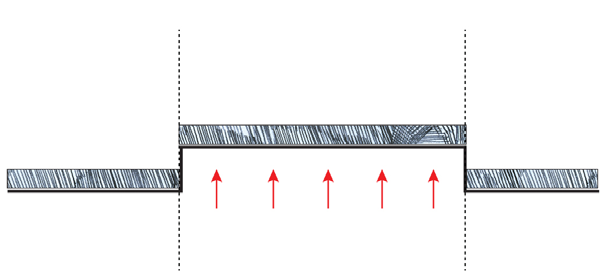

ELEVATION OF PUBLIC GROUND PLANE

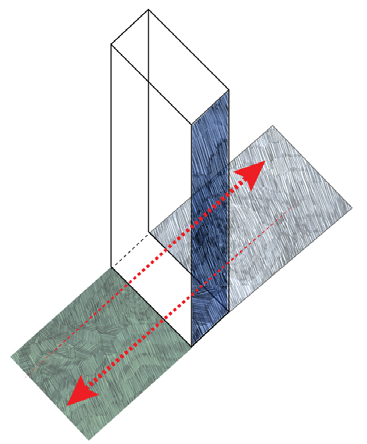

TRANSITIONING BETWEEN PARK AND STREET (TWO FRONTS)

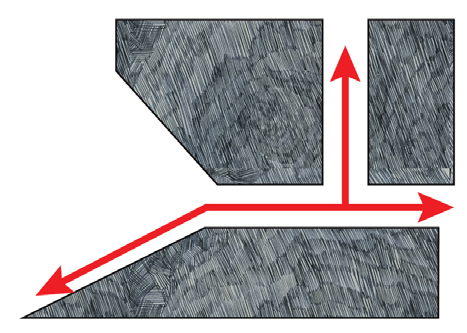

ACTIVATION OF INTERSTITIAL SPACE

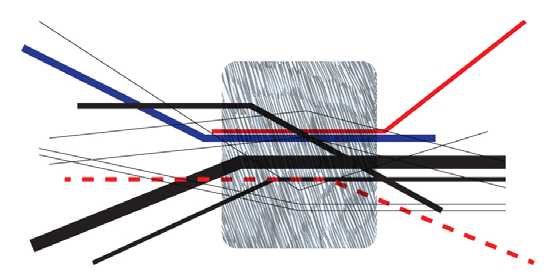

TECTONIC POSSIBILITIES OF PROGRAMMATIC OVERLAPS

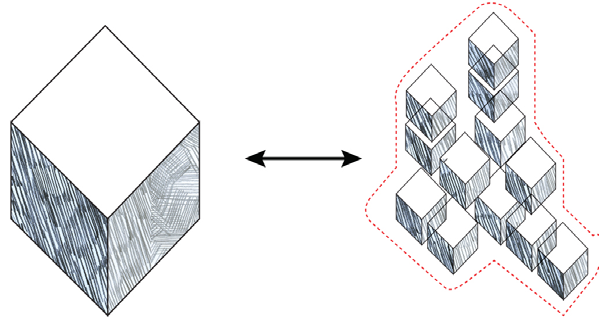

INDIVIDUAL VERSUS COLLECTIVE FORM

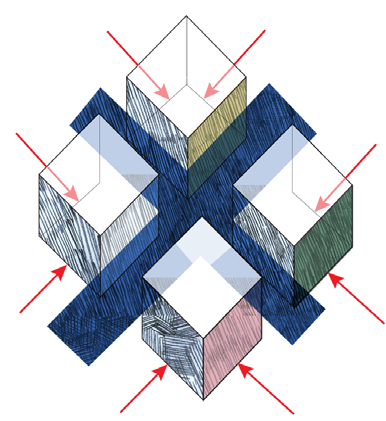

COMPRESSION OF DIFFERENT TYPES

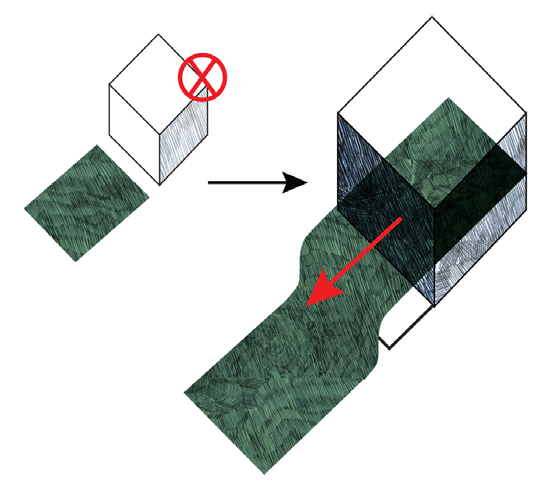

FORMAL ENGAGEMENT WITH LANDSCAPE 


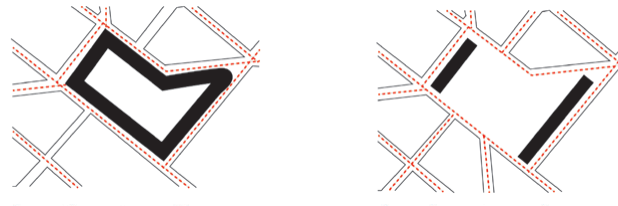

4
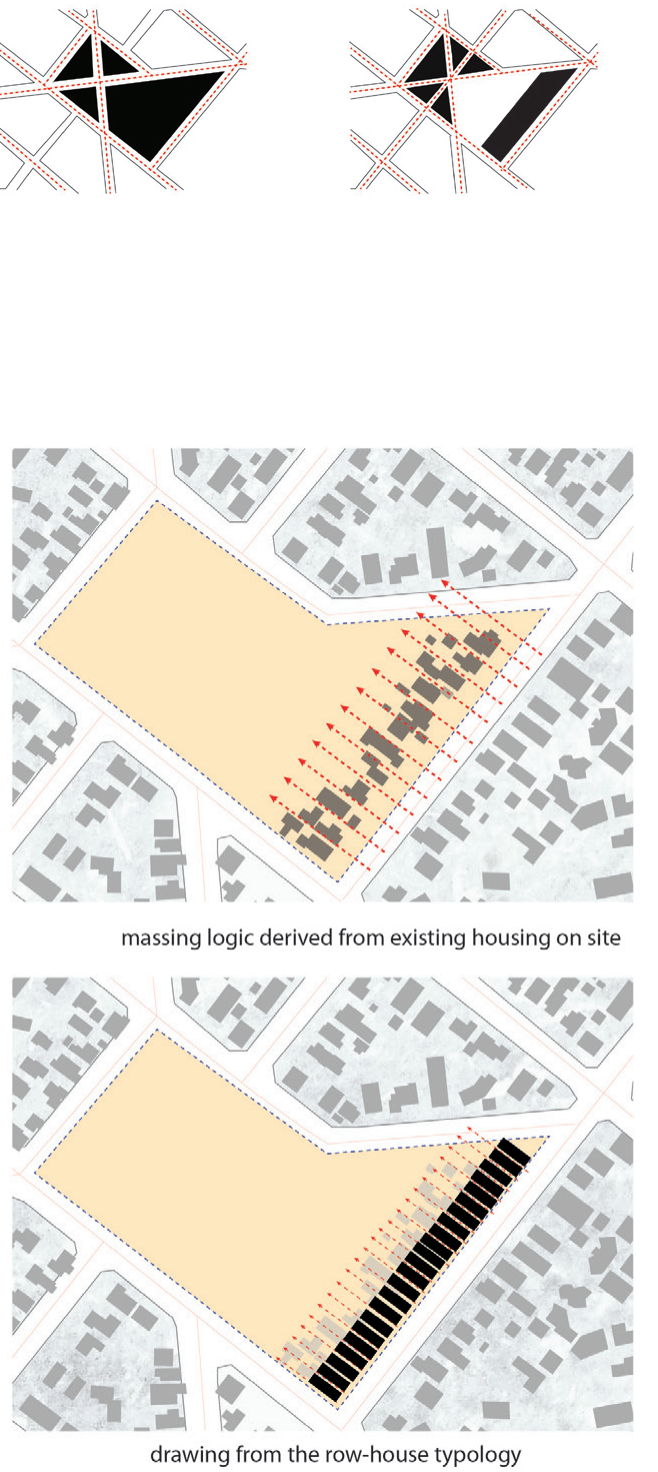

$\wedge>$

Figure 4 : Part to Whole: figure-ground studies in the early design stages experimented with individual versus collective form. These was informed by the immediate context (the building grain) on the one hand, but was also a means of fragmenting of building form (as a gesture) - to engage the project site and wider context in a certain fashion. Divisions between different elements as seen here were enabled here through slicing, striating and shifting along the site's edge, for example.
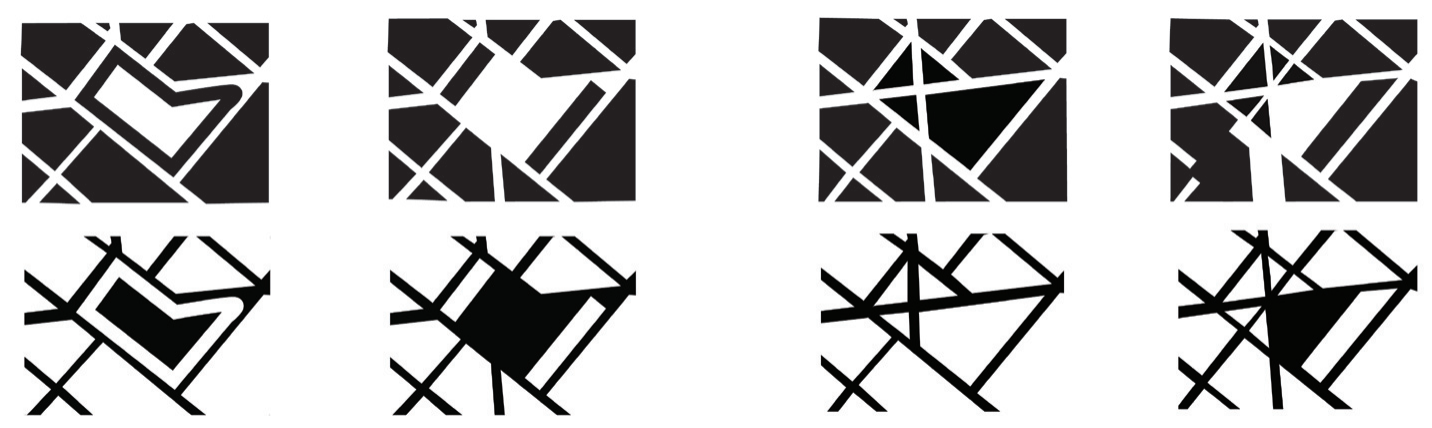

र
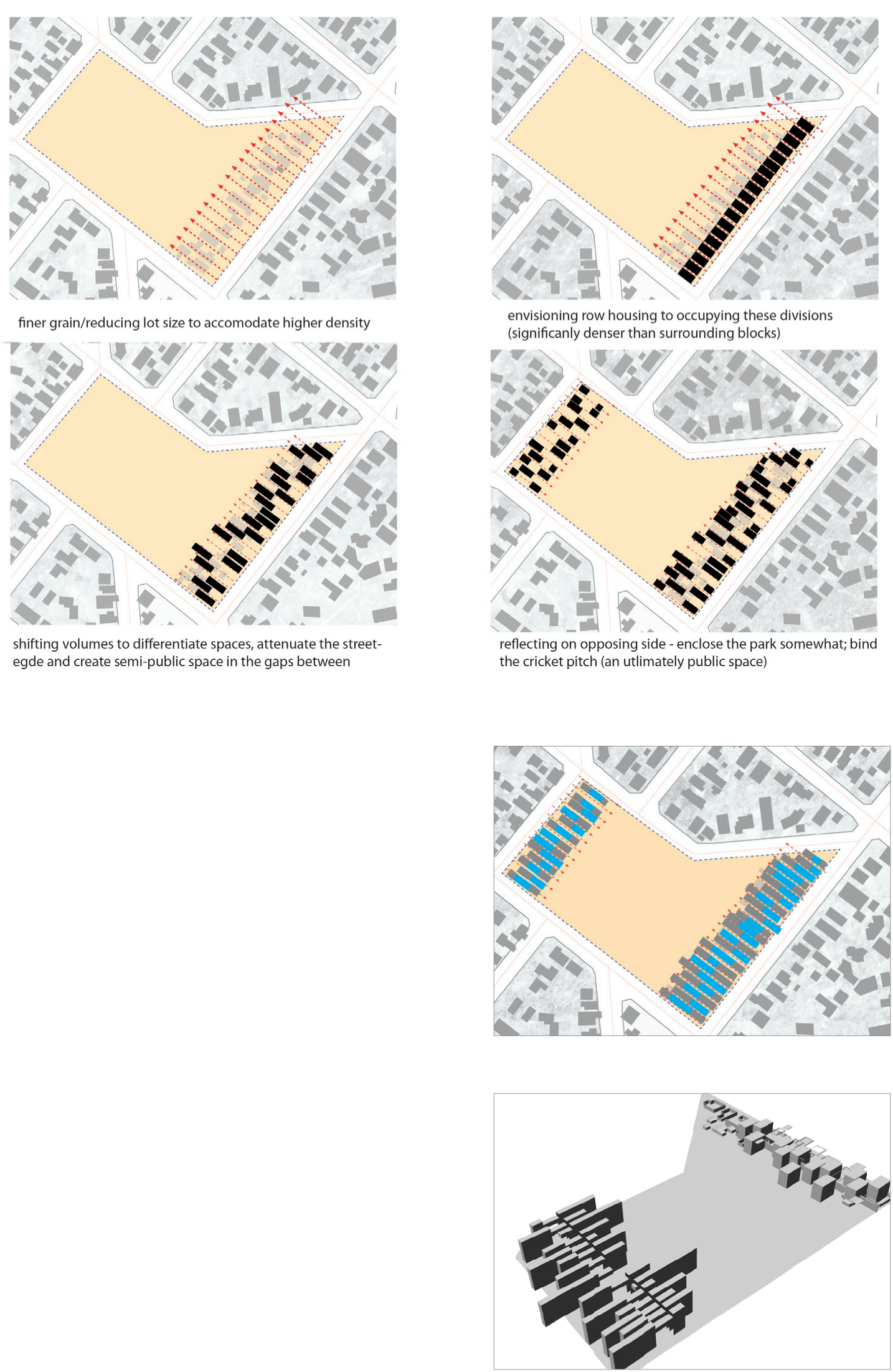

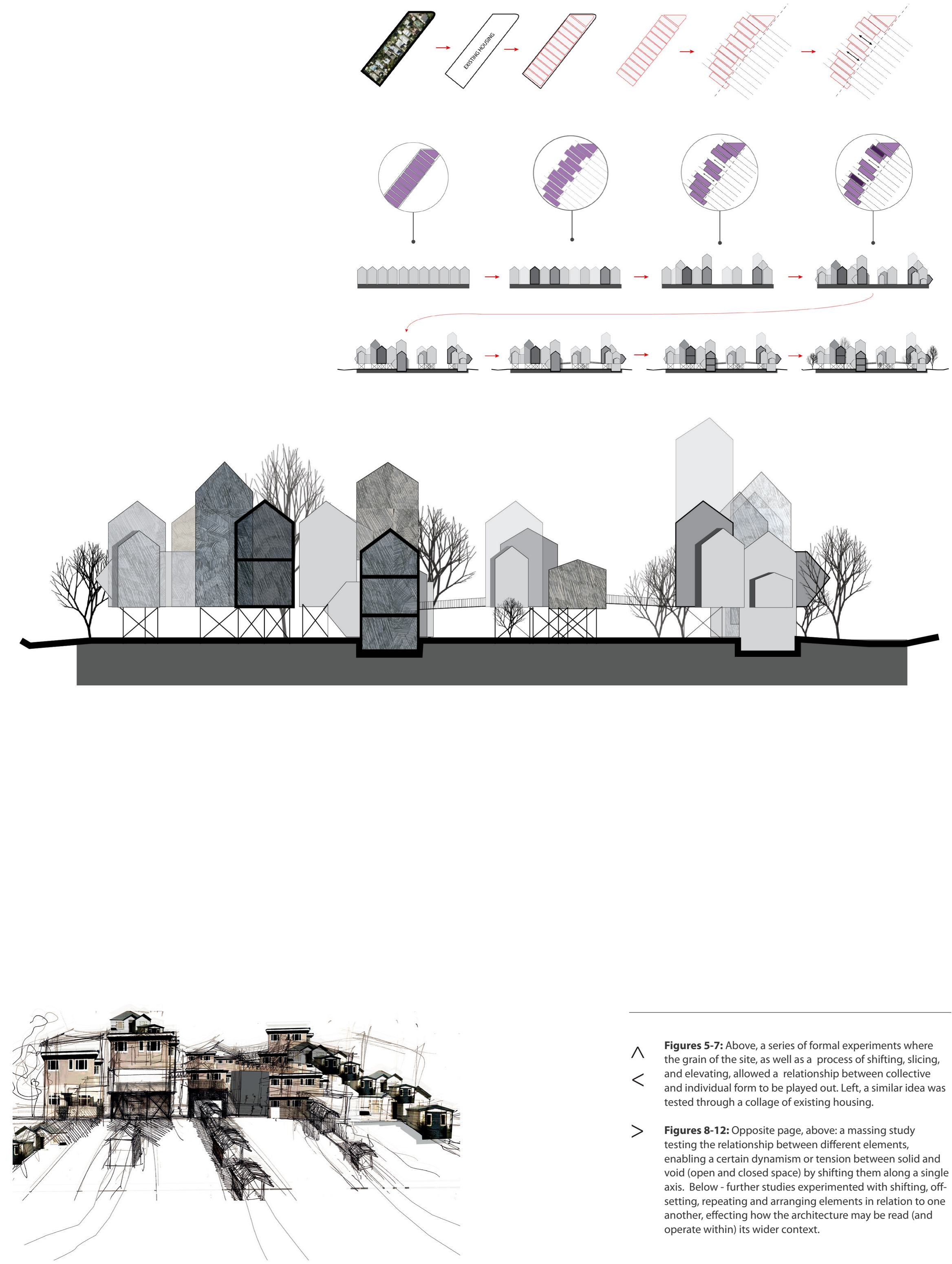

$\wedge$ Figures 5-7: Above, a series of formal experiments where the grain of the site, as well as a process of shifting, slicing and elevating, allowed a relationship between collective and individual form to be played out. Left, a similar idea was tested through a collage of existing housing.

$>\quad$ Figures 8-12: Opposite page, above: a massing study testing the relationship between different elements, enabling a certain dynamism or tension between solid and void (open and closed space) by shifting them along a single axis. Below - further studies experimented with shifting, offsetting, repeating and arranging elements in relation to one another, effecting how the architecture may be read (and operate within) its wider context. 


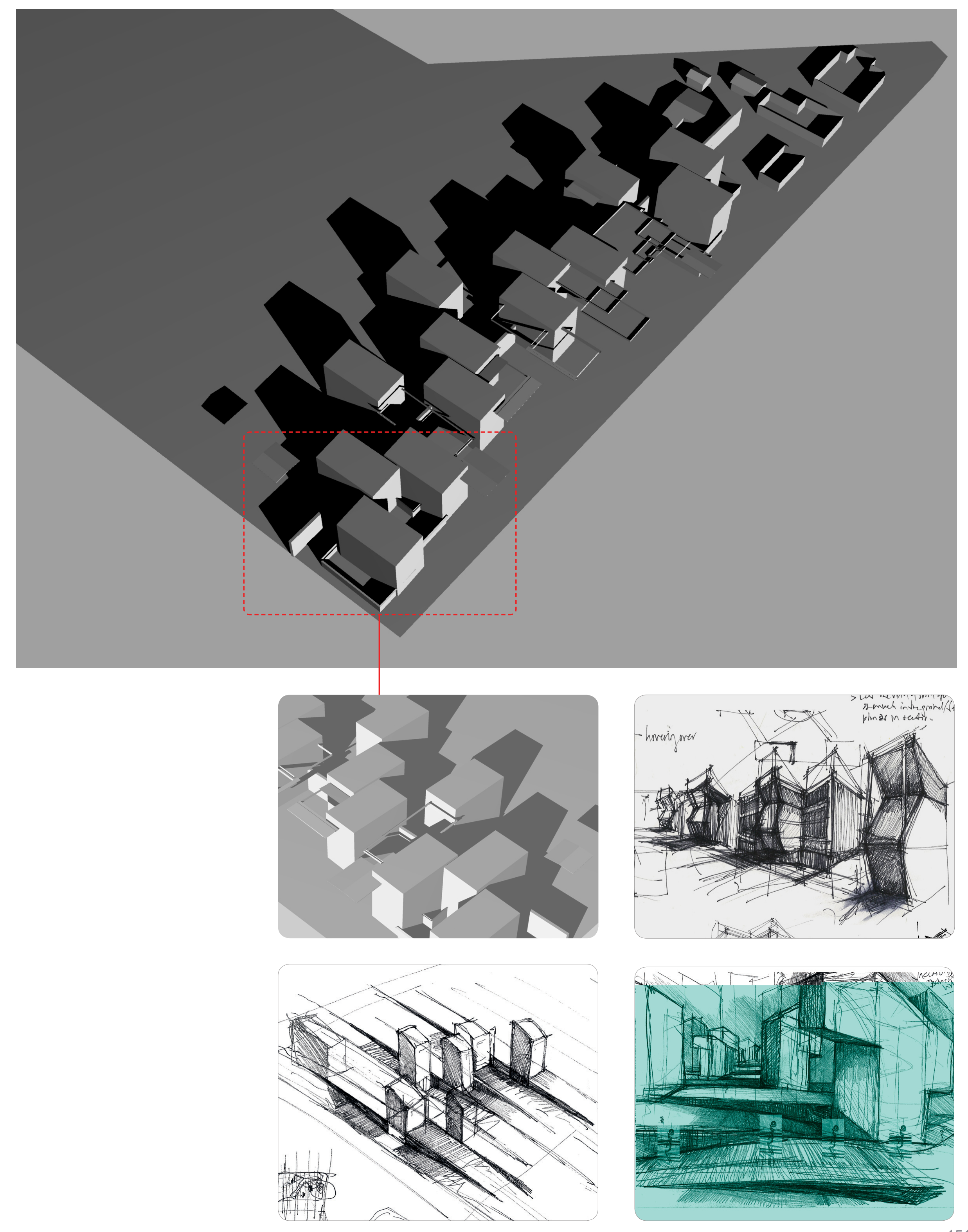




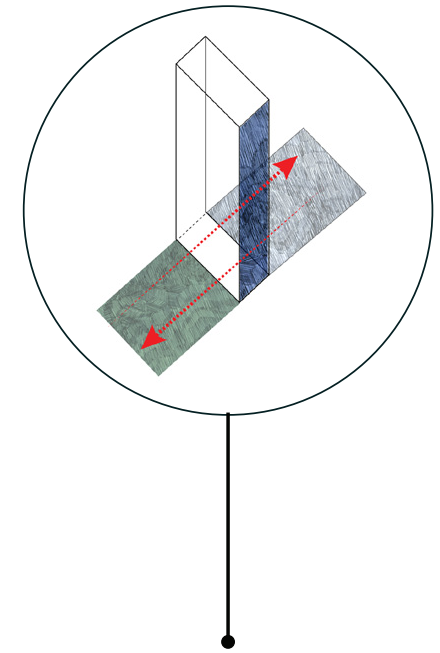

FORMAL MOVE 2

\section{TRANSITIONING}

BETWEEN STREET AND PARK

As a crucial formal gesture, the design processes explored how the architecture could engage both 'street' and 'park' with equal conviction and effectively operate as a medium between them. The fact that these two conditions were understood to necessitate differing architectural responses (on account of their distinct social and cultural connotations as well as their more physical character) was treated as a platform to investigate a fundamentally two-sided formal gesture.

This was tested through massing studies which sought to present each side with a different formal condition, yet allow them to overlap, intersect and relate to one another. Incisions (voids) through the building experimented with how it might attenuate the edge (rather than present a boundary) - and thus act as a formal, spatial and visual negotiation between the two contexts. (see figures 13-16) Notably, this gesture largely gave way to programmatic ideas: rather than being dictated by them.

\section{PAPAN Arrin}

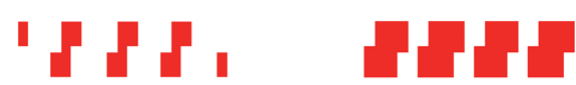

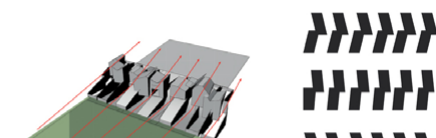

Anthy

cristin

HFFHH

thint
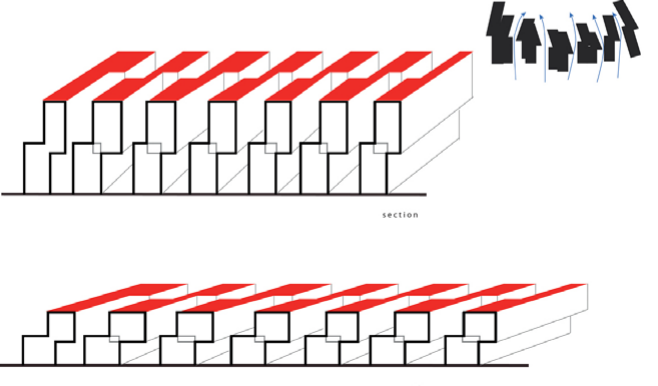

$\bigwedge$ Figure 13 : Above: a figure-ground and basic massing study which tested how park and street conditions might become linked through a series of off-set open spaces, off-set $>$ two sides to distinguish them.

Figures 14-16: Along the top and down the left hand side, early formal studies tested how the building might address park and street with different gestures: yet operate as a medium between the two. The possibility of linking through (with circulation) was tested as a formal/visual gesture shown in grey in these images. Right, treated as a forma opportunity, the need to engage both conditions led to experimentation with incisions and voids: cutting into the ground plane, and essentially through the built form. 

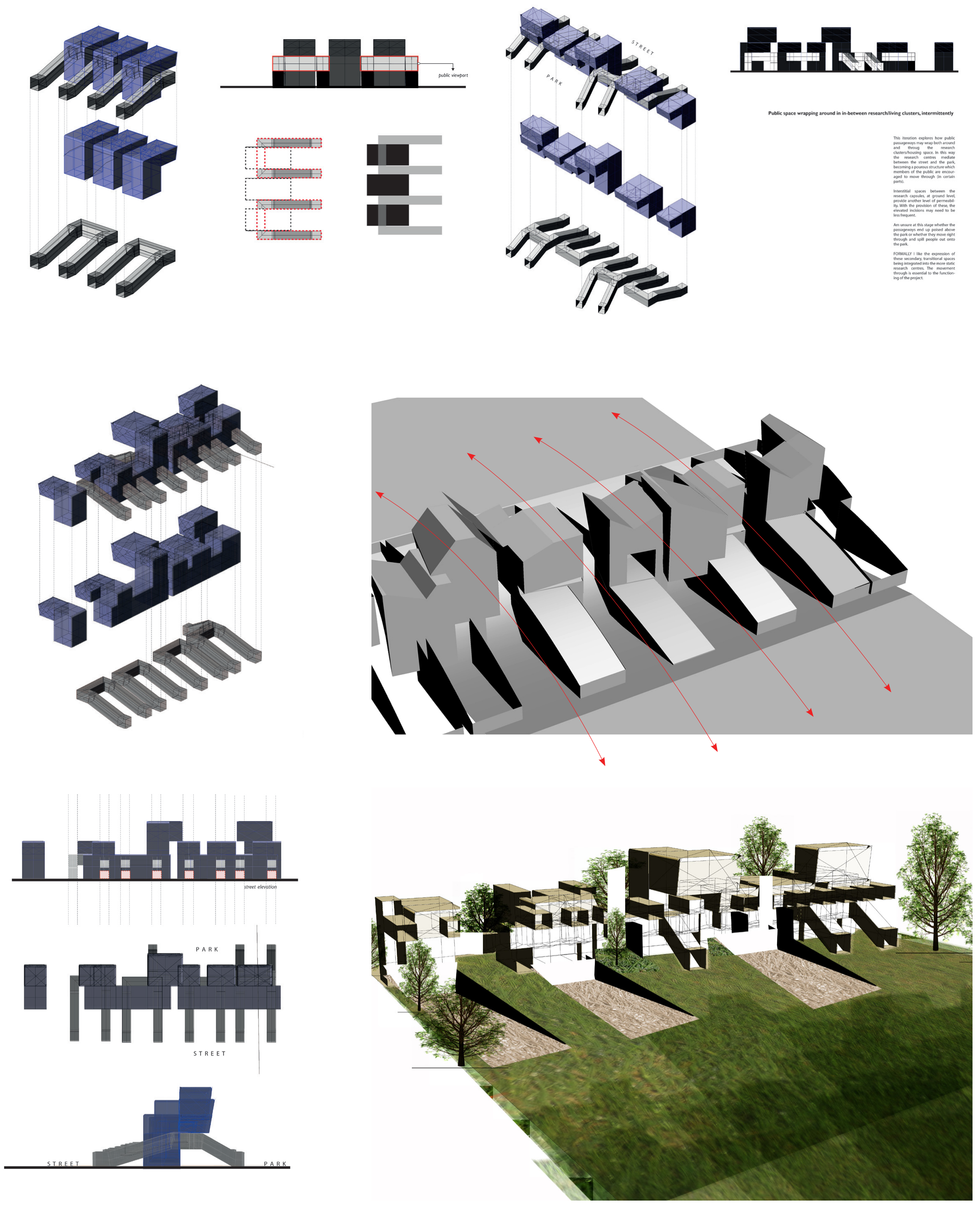

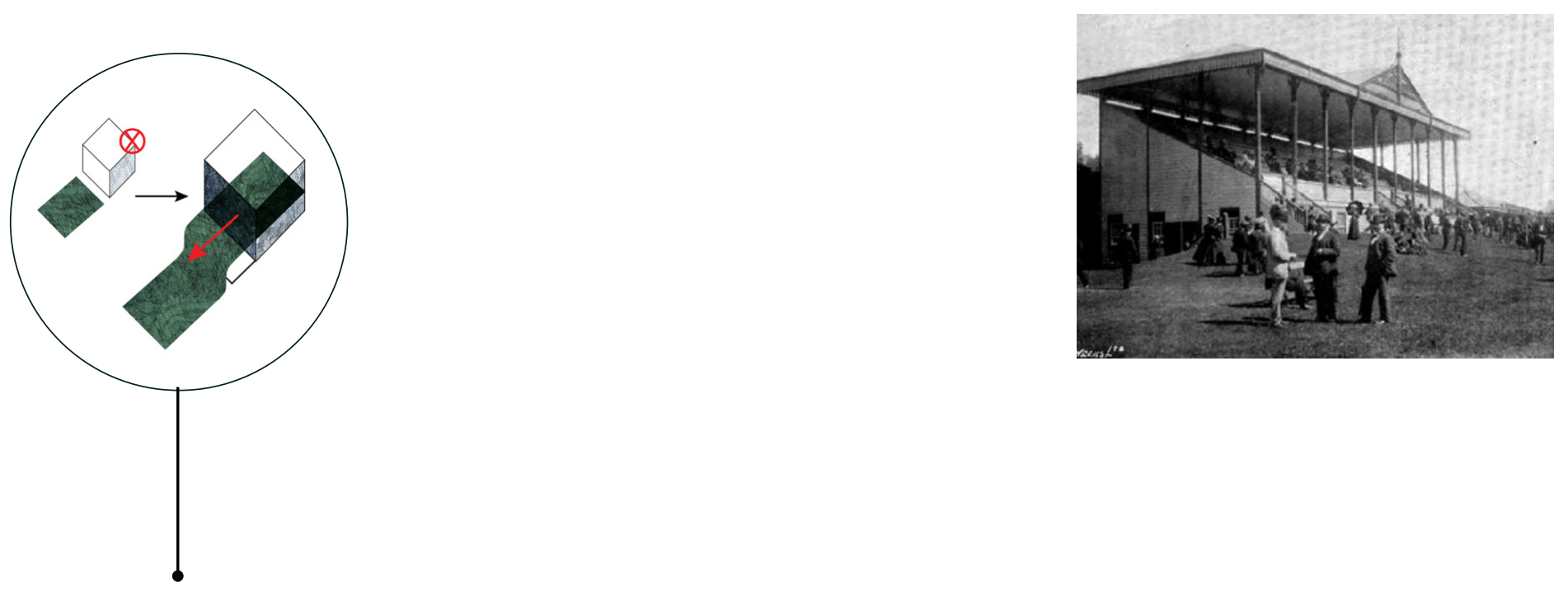

\section{FORMAL MOVE 3}

\section{FORMAL ENGAGEMENT WITH LANDSCAPE: THE USE OF TYPOLOGY}

As a means to formally engage the park, typological studies of the grandstand/pavilion were undertaken. These firstly studied a number of examples from around New Zealand, and considered their defining formal attributes: a clearly defined front and back; terraced on one side to meet the ground plane, overhung by an articulated roof structure; an abstract geometric profile; symmetry on the front elevation; solid-void relationships in section that are typically extruded along one axis, for example. (see figure 17)

These observations informed a series of sectional, elevational and massing studies: explorations to 'advance' the typology. These investigated how the formal attributes of the grandstand type might be brought to bear and manipulated on the site; and moreover, how the uni-directionality of this type might be challenged - in order to formally and spatially engage the street as well as the park. Furthermore, these explorations tested the interiorisation of the grandstand - given that it largely operates as an external structure. (see figures 18-20, overleaf)
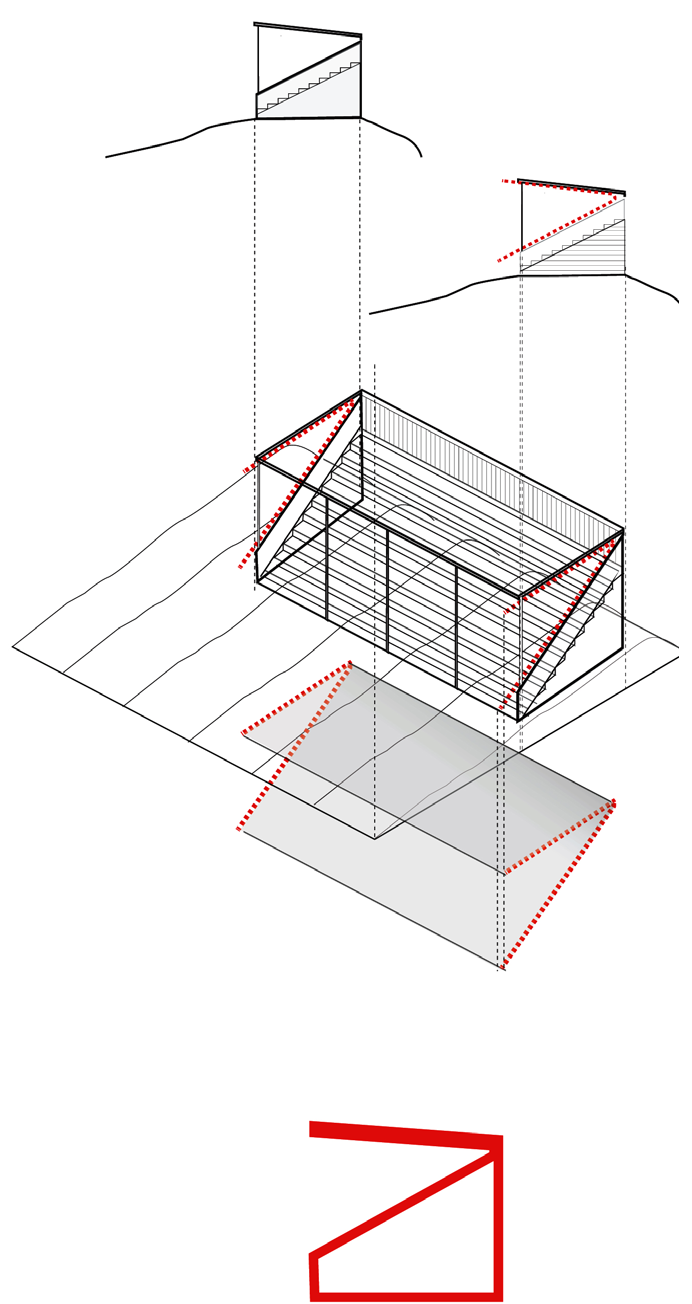

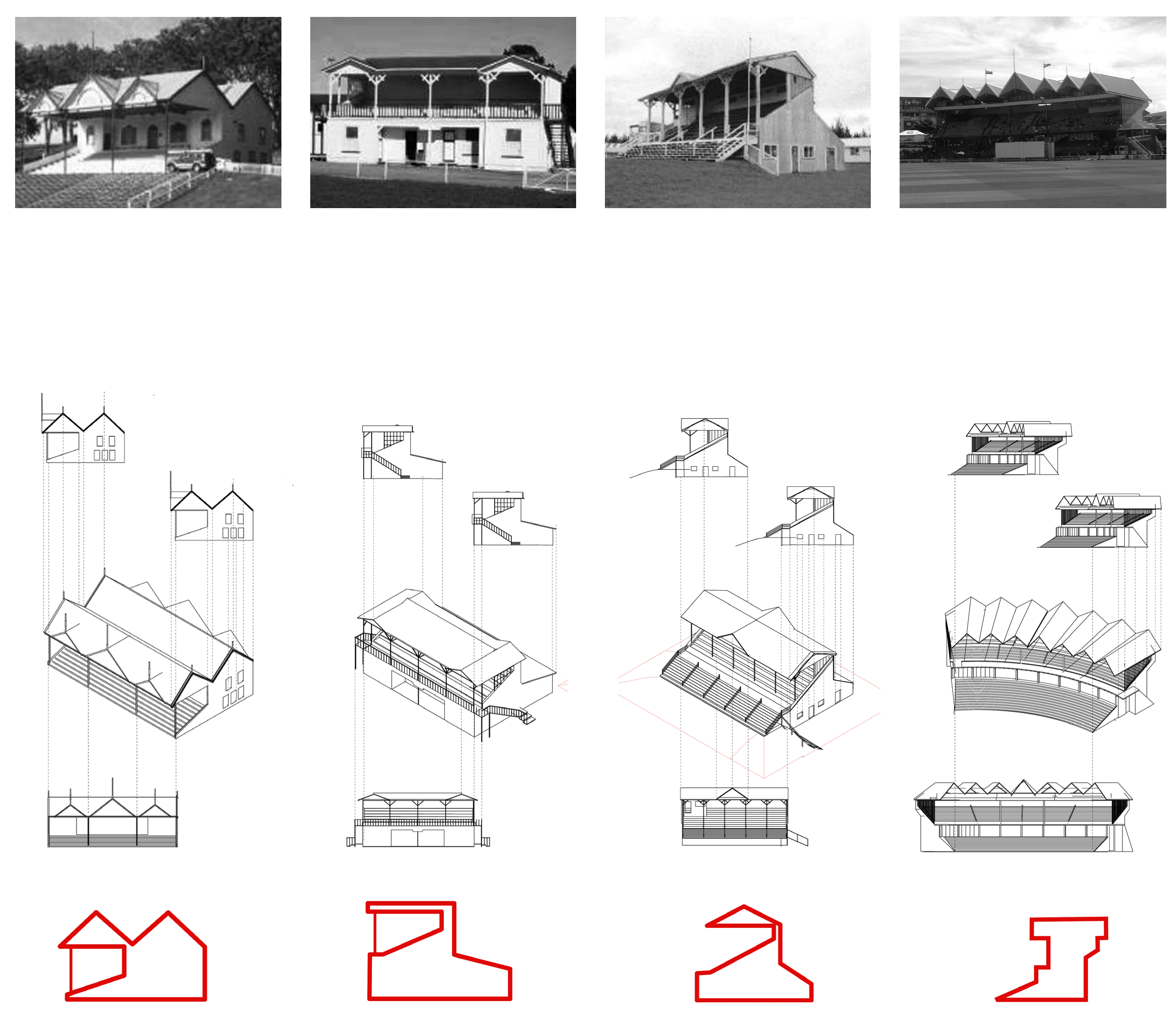

Figure 17 : Studies of the grandstand type: from left: Napier Figure 17: Studies of the grandstand type: from left: Napier Pavilion (Auckland 1898), Eketahuna Grandstand (ManawatuWanganui 1913), Kumara Racecourse grandstand (18871901), RA Vance Stand, Basin Reserve (1979). The abstracted elevations (bottom row) start to reveal the massing of this building type as a solid with a void subtracted. Each of these profiles presents a strange geometric language and a clear asymmetry (in contrast to the symmetry of the front elevation.) 

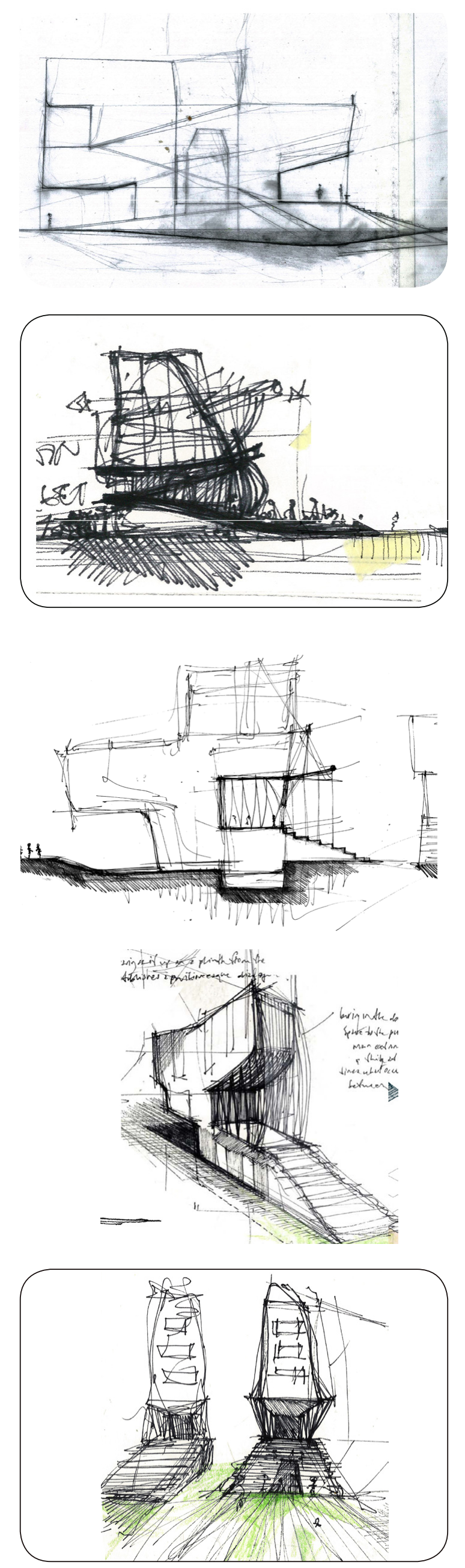
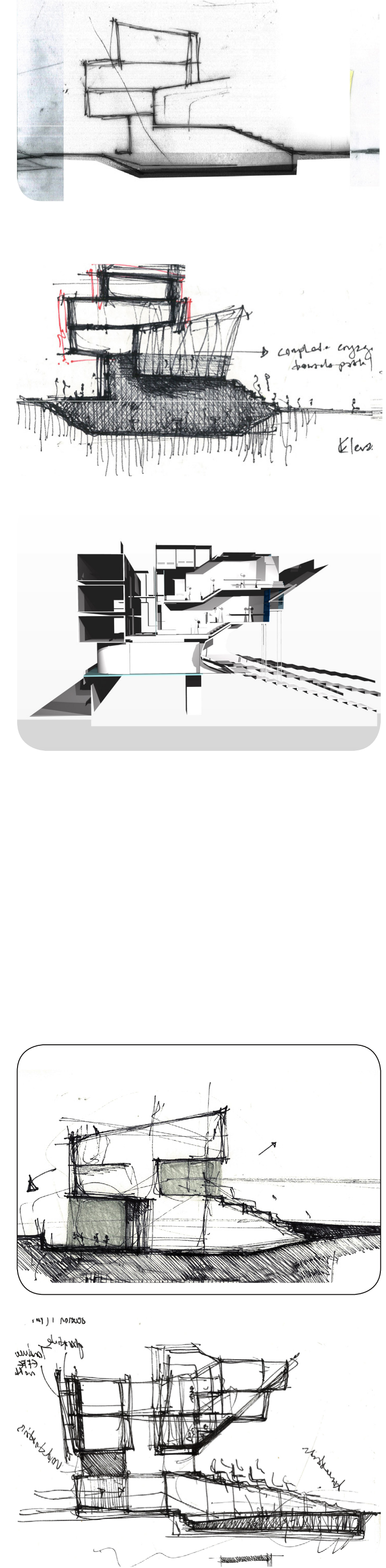
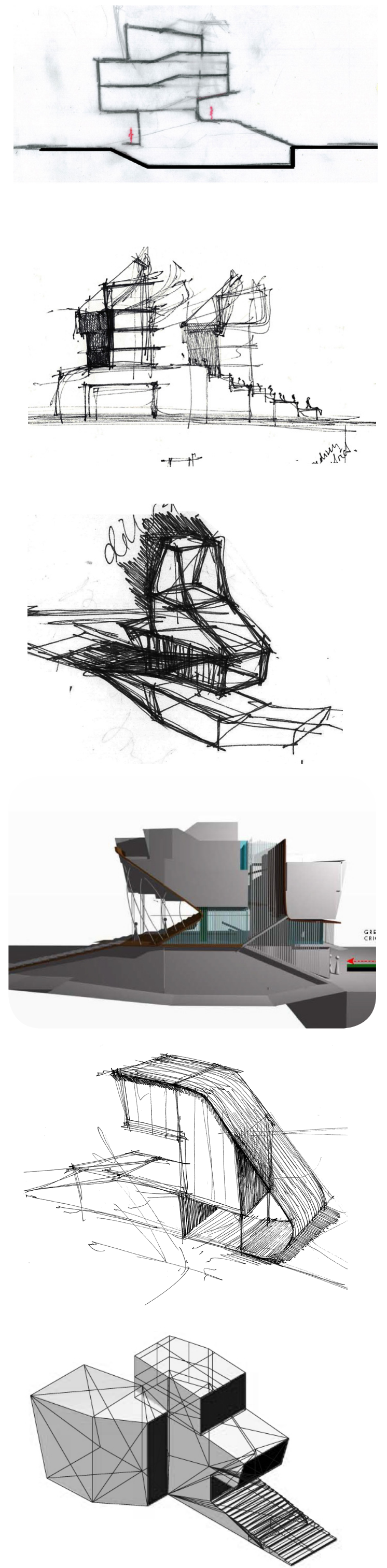

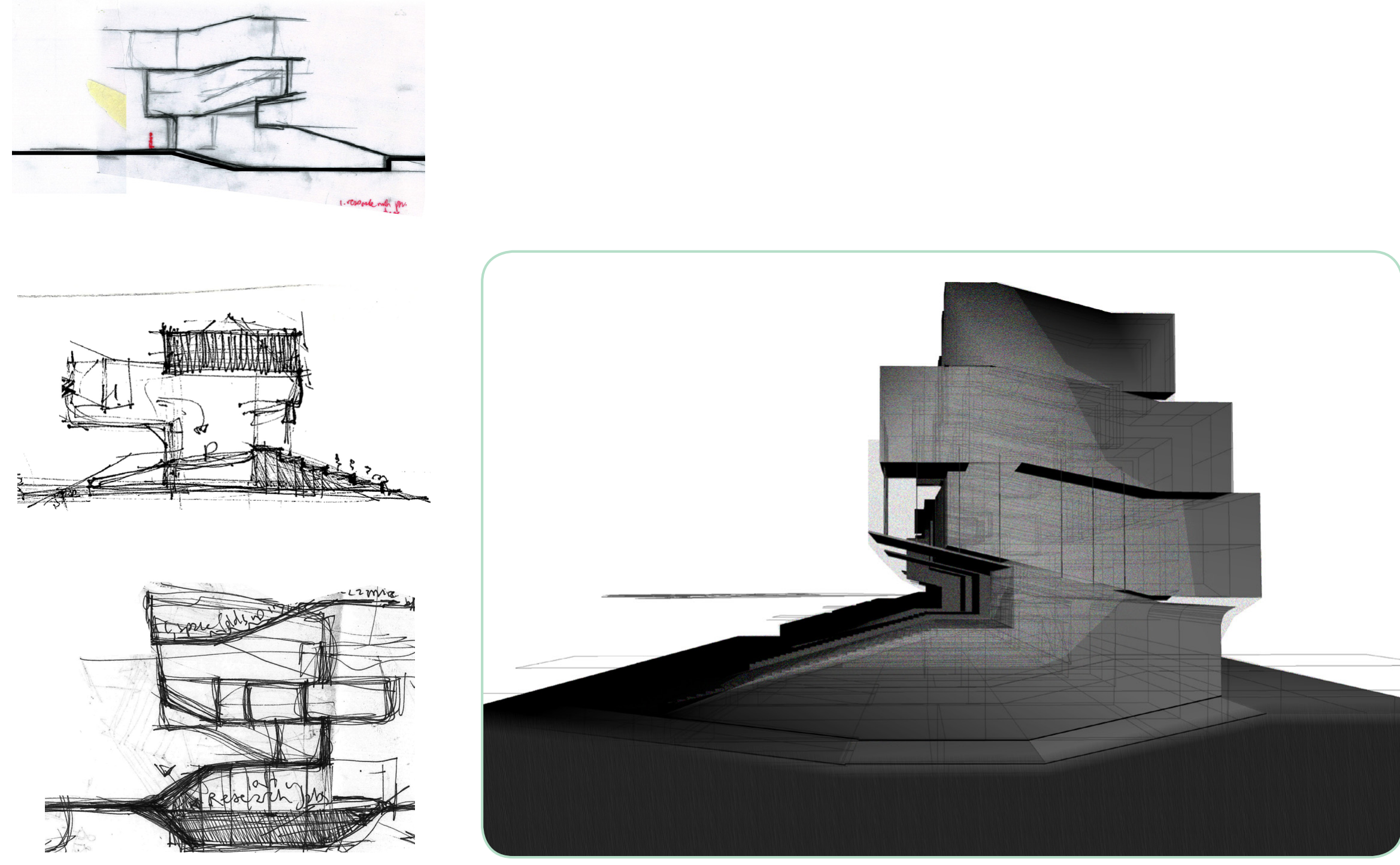

$<$ Figure 18: Left (all) Sectional, elevational and perspective studies explored derivations of the grandstand type, voiding out a mass, a terraced form to meet the ground plane, dramatic overhangs; and the interiorisation of the grandstand (whereby the overhanging roof structure may become a cantilevered mass, or a layering of volumes for example). These explorations tested the typically uni-directional nature of the grandstand: considering how it might directly engage the street (not just as a backside).

$\bigwedge$ Figures $19+20$ : Above: a layering of forms enables a transitional gesture between park and street to emerge; with terraced forms meeting the park. Below, the typological studies were explored in relation to smaller scale masses: separated and repeated along the site edge, as individual pavilions.
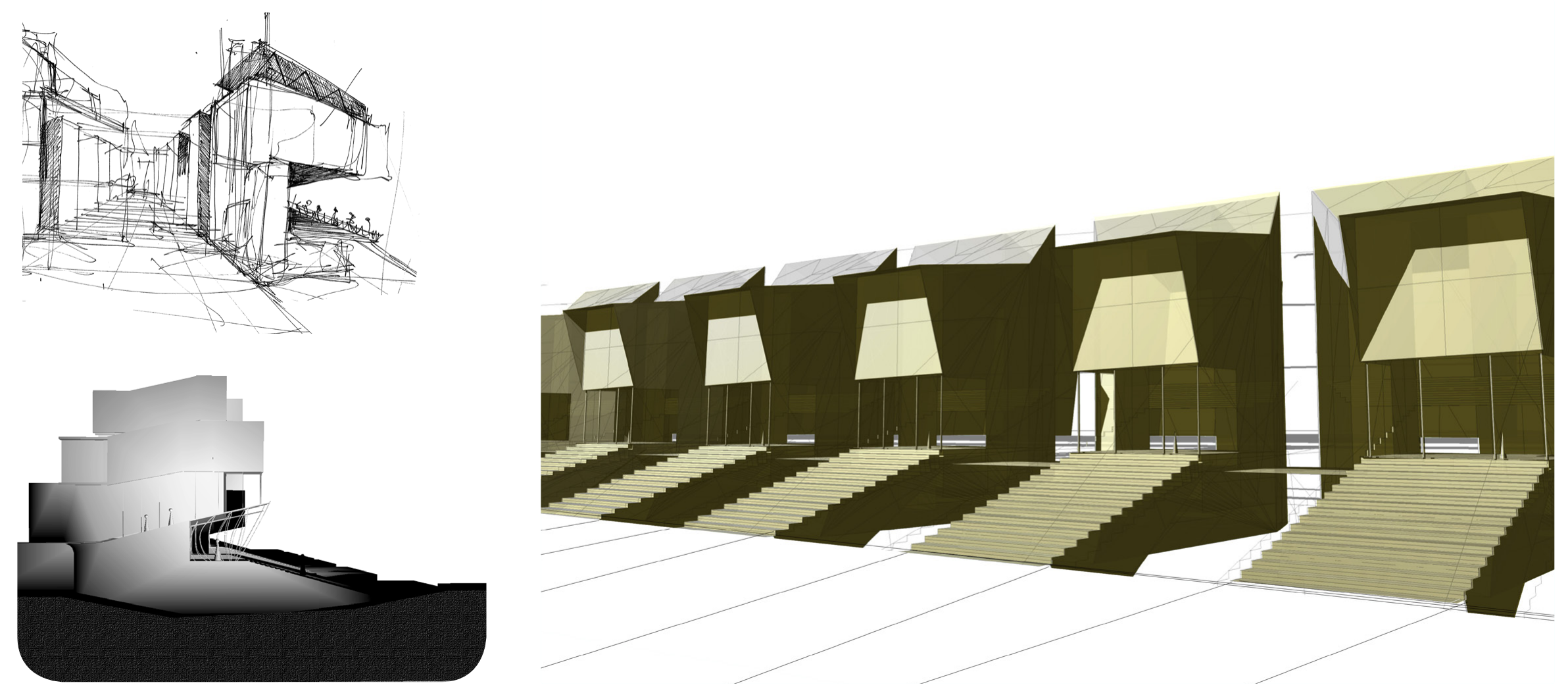

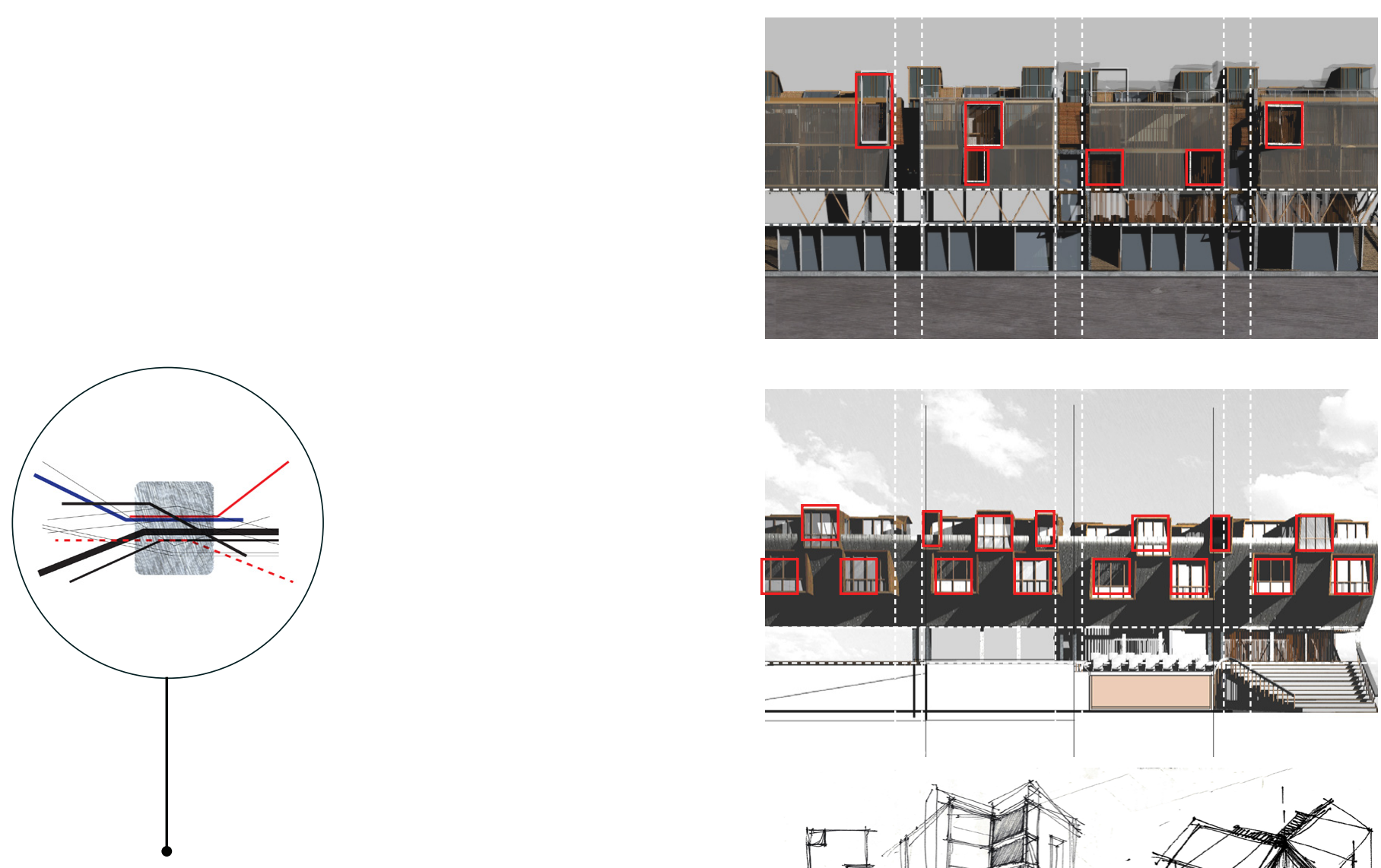

FORMAL MOVE

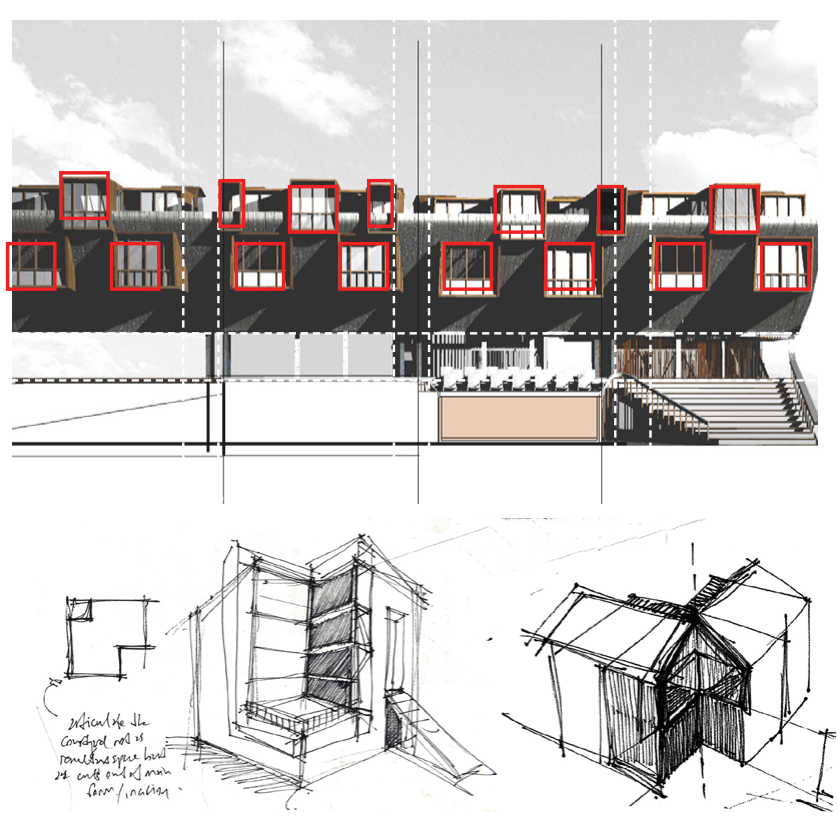

\section{TECTONIC POSSIBILITIES OF PROGRAMMATIC OVERLAPS}

The design processes considered the proximity of 'living', 'working' and 'playing' programs (or cultures) within an emerging sub-centre context as an explicit formal opportunity: testing how these might lead to a distinct tectonic, spatial and visual articulation and expression. Strategies experimented with how different scales, proportions, and configurations of volumes and masses might relate to one another visually; what sort of spaces may result between them; and what this could mean for the building in its entirety. Particularly these a worked with a more 'densified' architectural language as a certain formal gesture, where differing elements may be composed and held in relational tension. (see figures 21-25)

Figure 21 : Elevational studies tested a visual /formal expression that may bring together public/private/dense/ rarefied programmes, in a unified gesture. These voided out spaces from a larger whole; tested different proportions and scales of masses in relation to one another (and how they might be articulated as separate), and played with the repetition of elements as a compositional gesture: reflecting ideas about new modes of habitation/ shared-living. Below: articulating interfaces as a subtraction of forms.

Figures $22-25$ : Design processes experimented with bringing together a densified architectural language (smaller elements, for example), with large scale public volumes -and the relational tension arising between these - articulated formally. The upper image shows a textured continuation of different sized forms stacked on top of another to a certain visual and formal effect. The middle elevation considers how 'live' work and play might inform an arrangement of spaces across the entire site: the large open space in the middle juxtaposed with the densified edges, for example. 

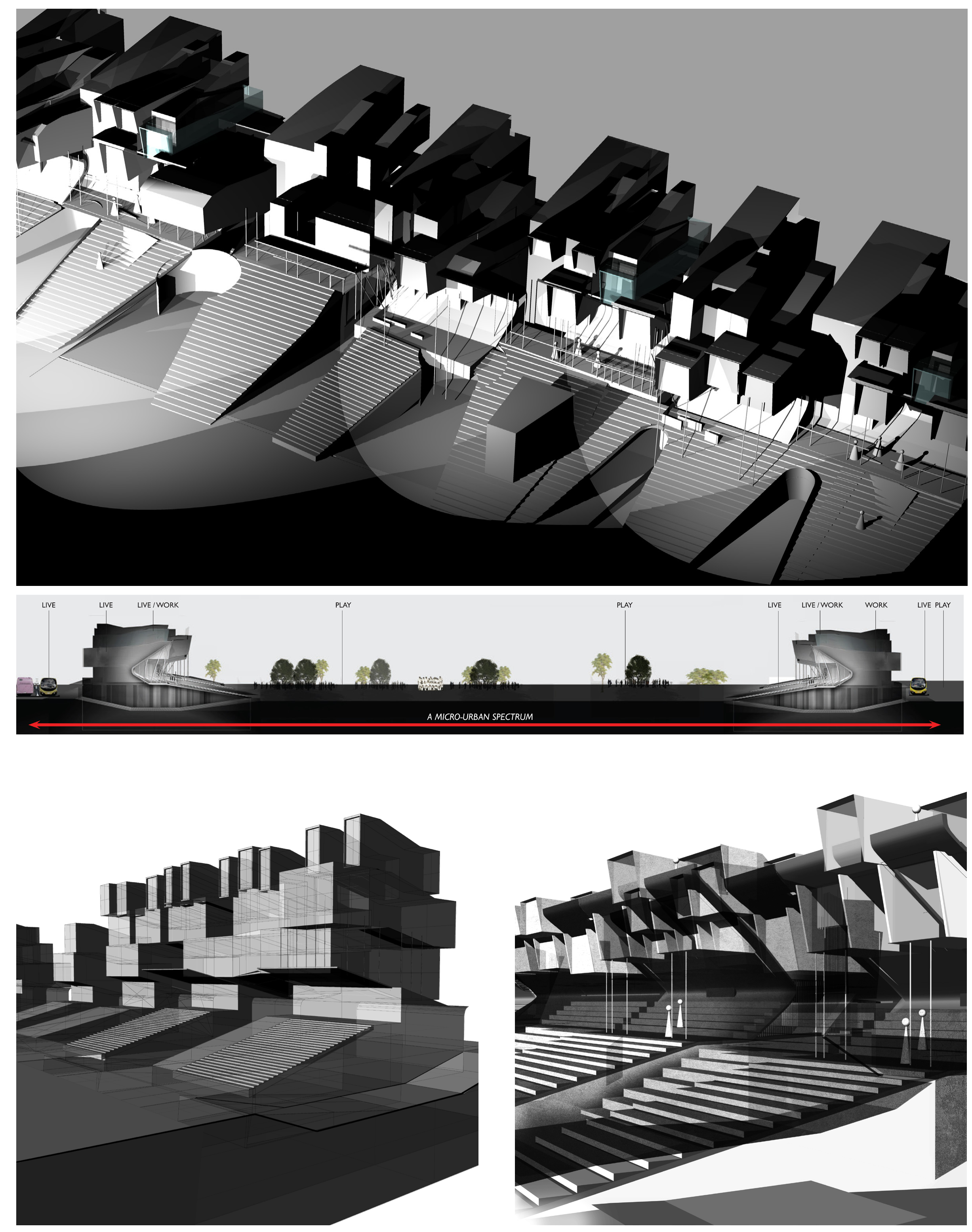

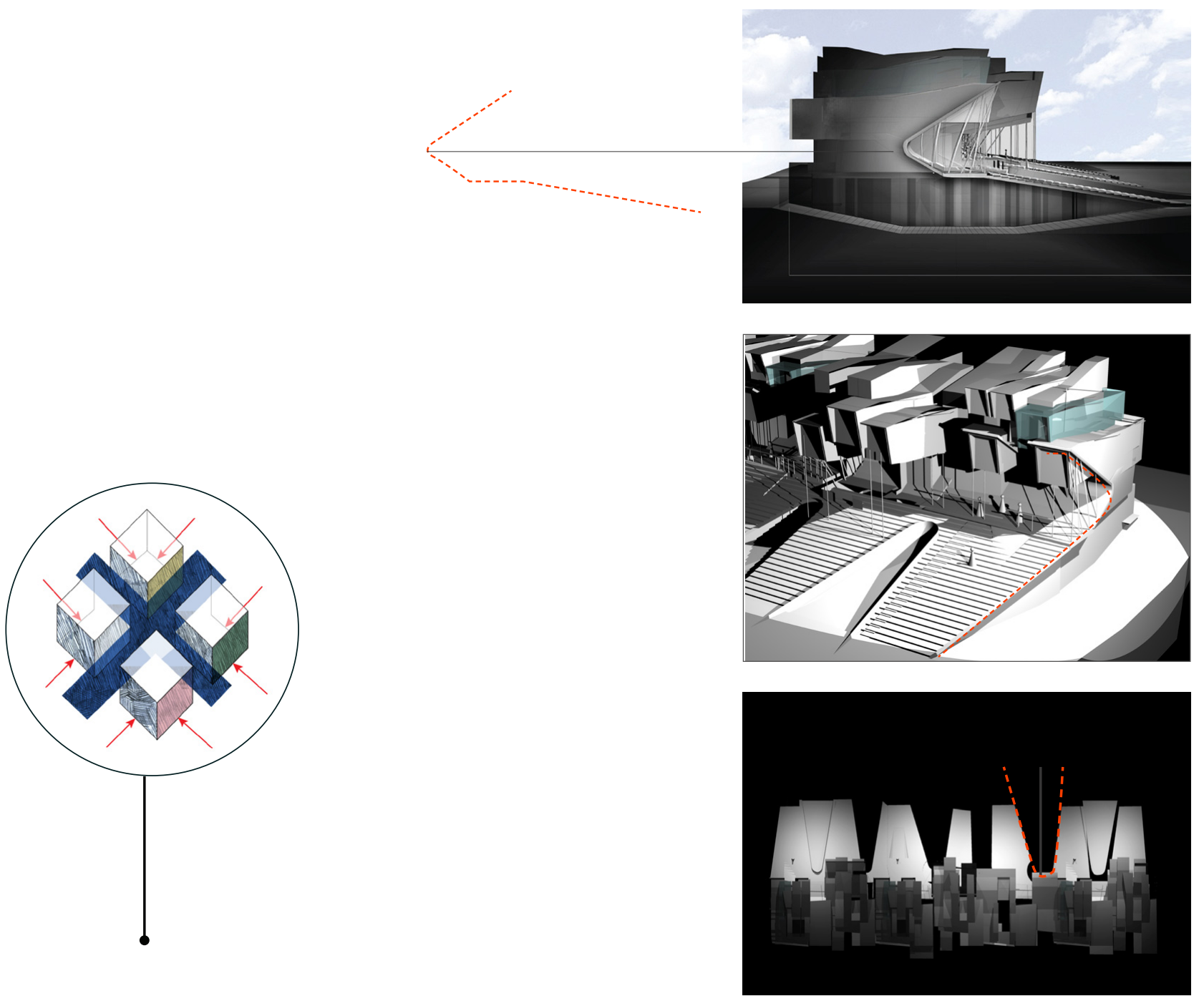

\section{FORMAL MOVE 5}

\section{COMPRESSION OF DIFFERENT TYPES}

The design processes 'compressed'and interfaced the grandstand type with other architectural languages: particular that of medium density housing. This involved juxtaposing these different languages and forms, cutting one into the other - such that they might intersect and read as a unified formal gesture, yet one composed of differing parts. This effectively introduced a more informal densified and urban language to the typically more uniform front-elevation of the grandstand. Bringing these types together also had the effect of 'spatialising' the grandstand: enabling it to be tested further as a means to negotiate between street and park spatially and formally. (see figures 26-29)

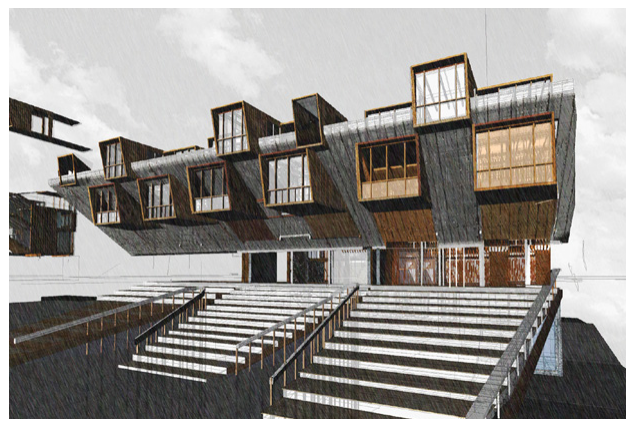

Figure 26: Compressing and interfacing the grandstan type with divisions derived from the street, as well as with a more densified urban architectural language, was explored as a formal gesture.

Figure 27 - 29 : Explorations into how one type might be 'cut' into the other as a clear visual formal and spatial expression (perspective); and as a means to 'densify' the grandstand for example (elevation). The section compresses multiple types into a singular gesture: of multiple stories, a rich interior and a modulated facade sunk below the datum. This enabled further a transition between street and park and challenged the unidirectionality of the grandstand 

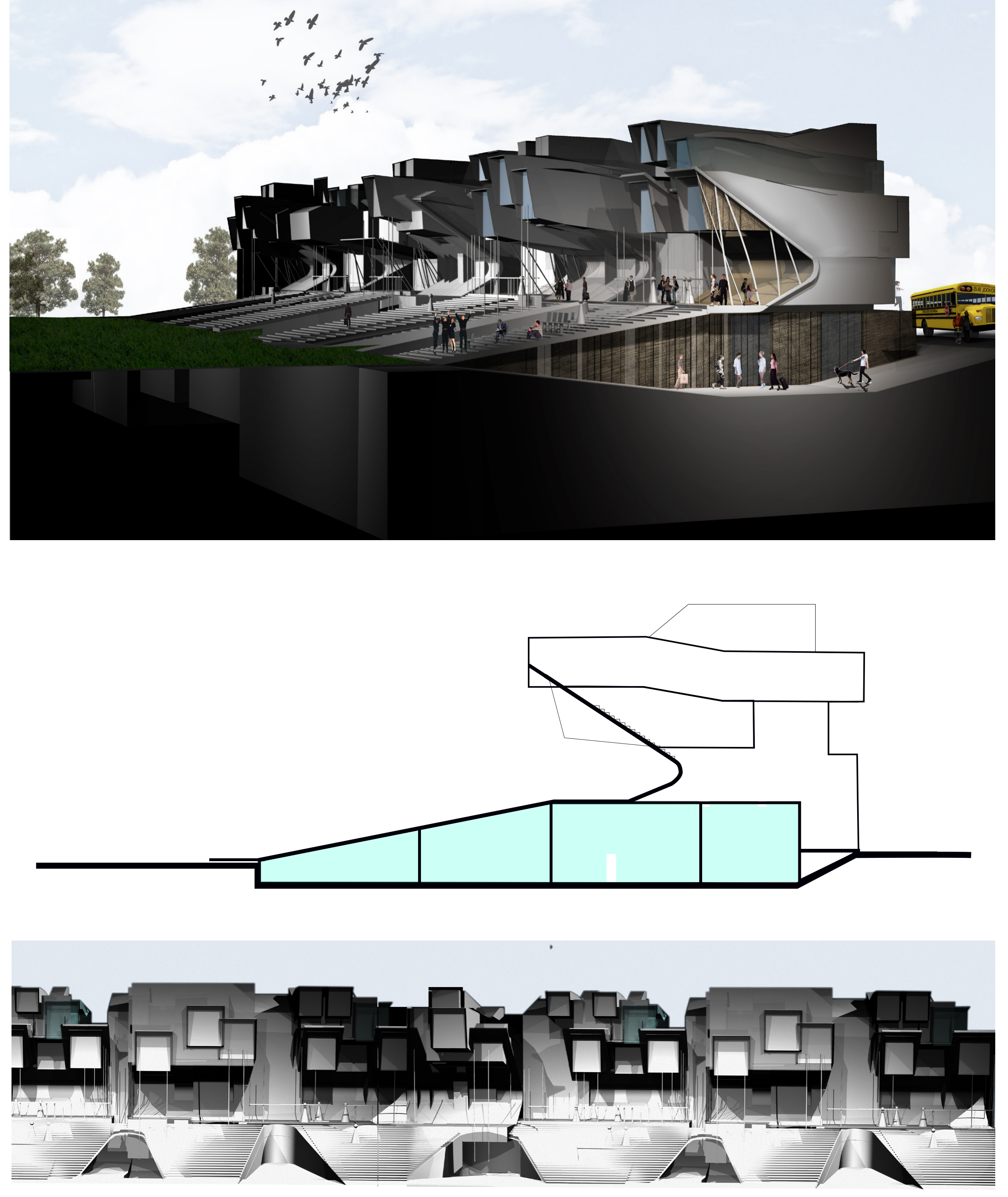


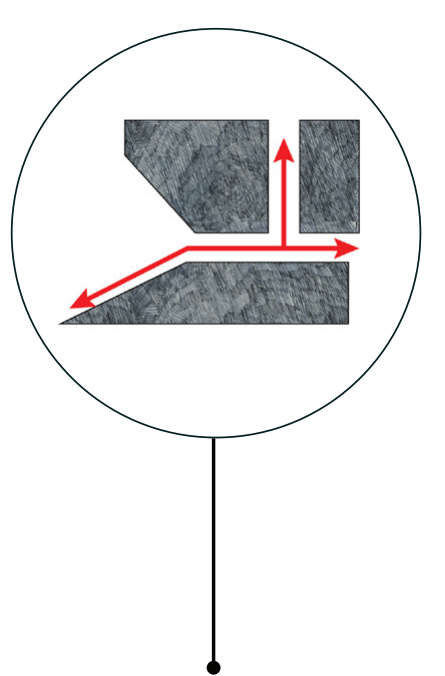

FORMAL MOVE 6

ARTICULATION OF INTERSTITIAL SPACE: SEPARATION OF ELEMENTS

The separation of different elements (and the articulation of the space between them) was explored in section, elevation and perspectiveand spatial configuration/organisation. Essentially treating the building into three primary masses, the activation and expression of this interstitial space allowed the exterior space of the park to enter into the architecture: visually, spatially and formally re-configuring the grandstand gesture, into a fully-interiorised, inhabitable and 'spatialised' architecture. In elevation, vertical interstitial space (related to the street divisions, and circulation) was also considered as a formal/ visual expression of separation.

The tension between masses and the resultant immediate space was explored in many different aspects - and to differing degrees - but came to effect the design in its entirety. (see figures 3033 )
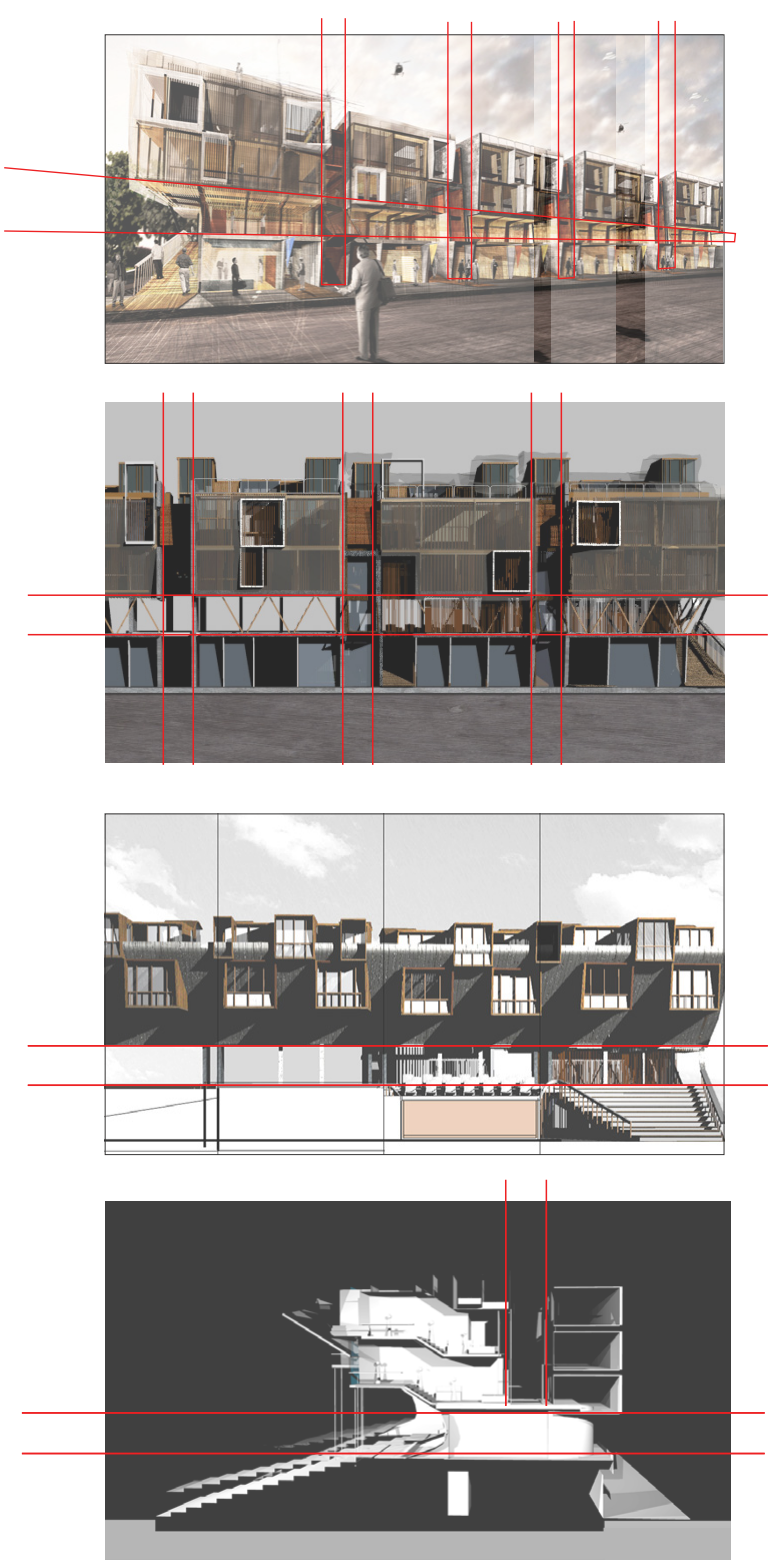

Figure 30 : Articulating the interstitial space (separation between elements) as a formal and visual gesture was explored in both street and park elevation - serving to unify the two sides; as well as in section.

$>$ Figures 31 - 33 : Above: a massing model where the building was considered as three main masses, separated by an interstitial space. This division was heavily explored in section, especially informing the configuration of the interior spaces (far right). It was also articulated the elevations; reading as 'carved out' space from most aspects (bottom left). 


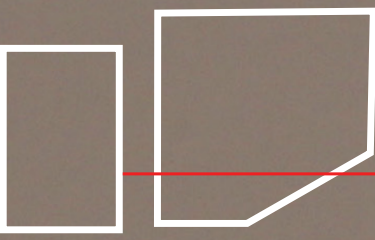

live

work
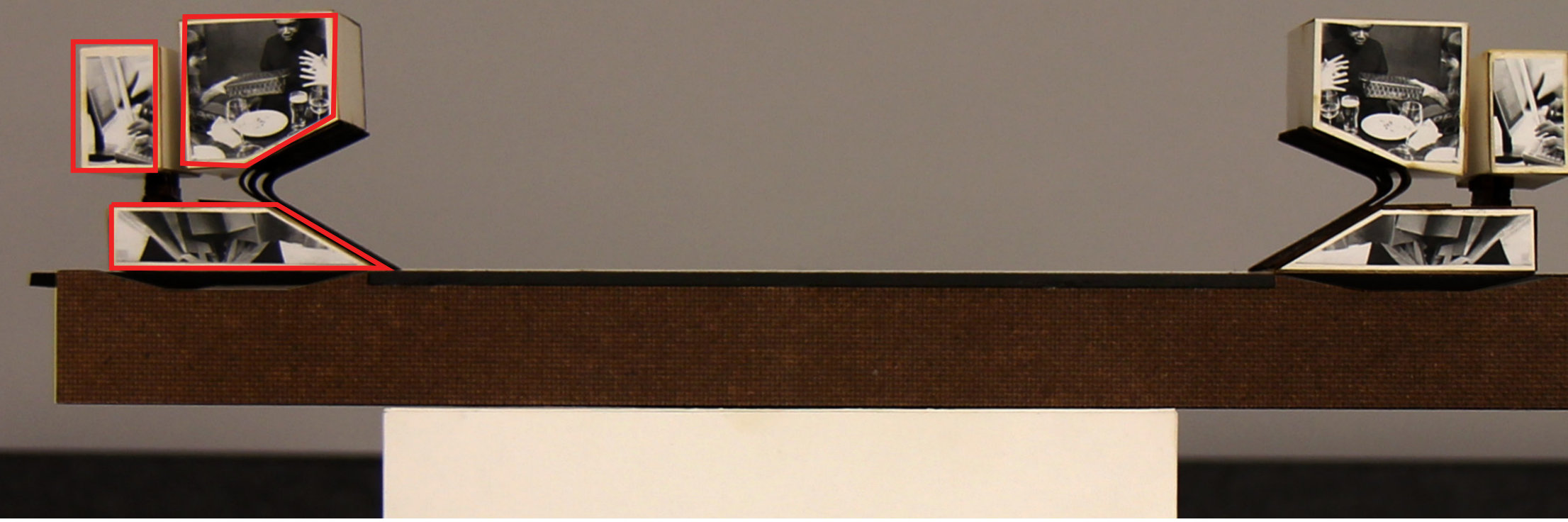

N. $=1$
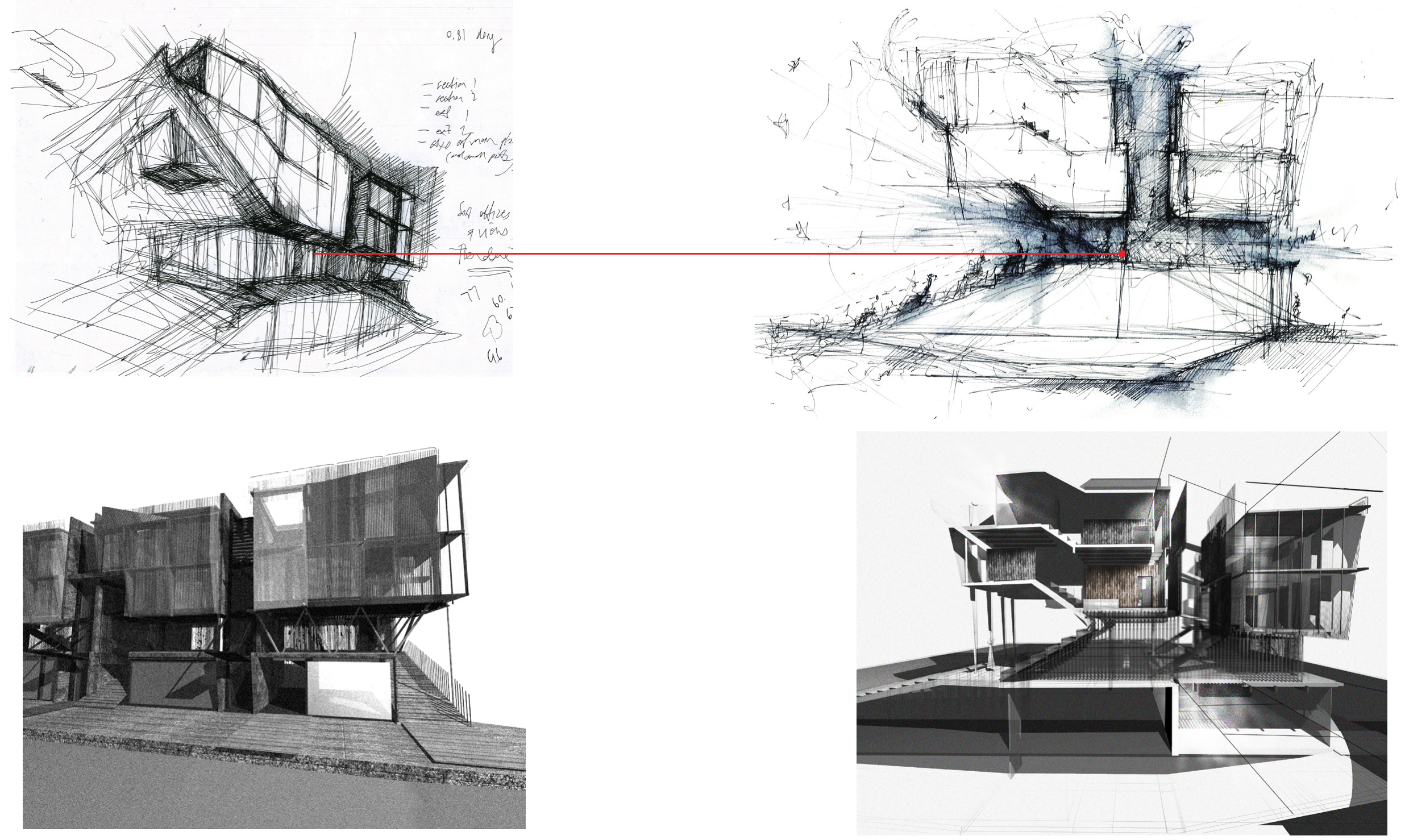


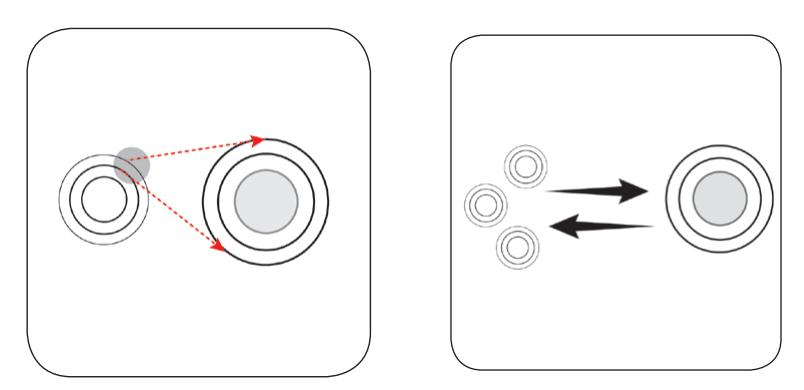

\section{A Radical, Contextual In- tervention [upon site]}

The project's engagement with site is enabled largely though the typological gesture (the grandstand and occupation of two opposing edges); the fundamentally two-sided address; and the part-to-whole explorations (responding to existing grain). Where the basic typological gesture permits a historical, cultural and social resonance with the site, it enables a contextual or even 'appropriate' intervention. Yet, injected with a more fragmented architectural language; divisions fundamentally derived from a street context; and a collection of varied public spaces at its base, this 'appropriate' gesture is radicalised somewhat: the grandstand permeated, punctured and reconfigured with an pervasive informality as it encroaches onto the park. Likewise, as it is staggered and pressed up against the street - unequivocally given a second side this more 'typical' relationship to site is departed from.

The formal intervention that results from this juxtaposition is both deeply contextual on the one hand- and yet radical and subversive on the other. Engaged as such, the park may considered as much a recreational or athletic space as one public or political; as suburban as it may be urban: indeed, neither village green nor town square but perhaps somewhere between the two - if not entirely different from both. (see figures 34-35)

In this way, the project might be seen to engage the urbanizing suburb as a place of inherent transition: distilling the tension between its former and emergent state (as suburb and urban-centre, effectively) within a single, engaged intervention on site.

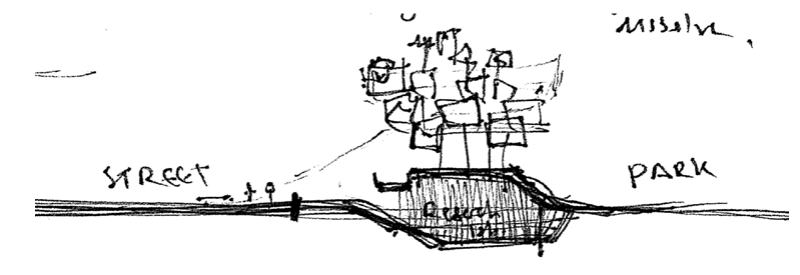

Figure 34 : Formalizing the Transition: By exploiting and enacting the tension between park and street as a formal gesture; the architecture is able to engage with the context more broadly; imparting idea about its transition from suburb to urban centre.

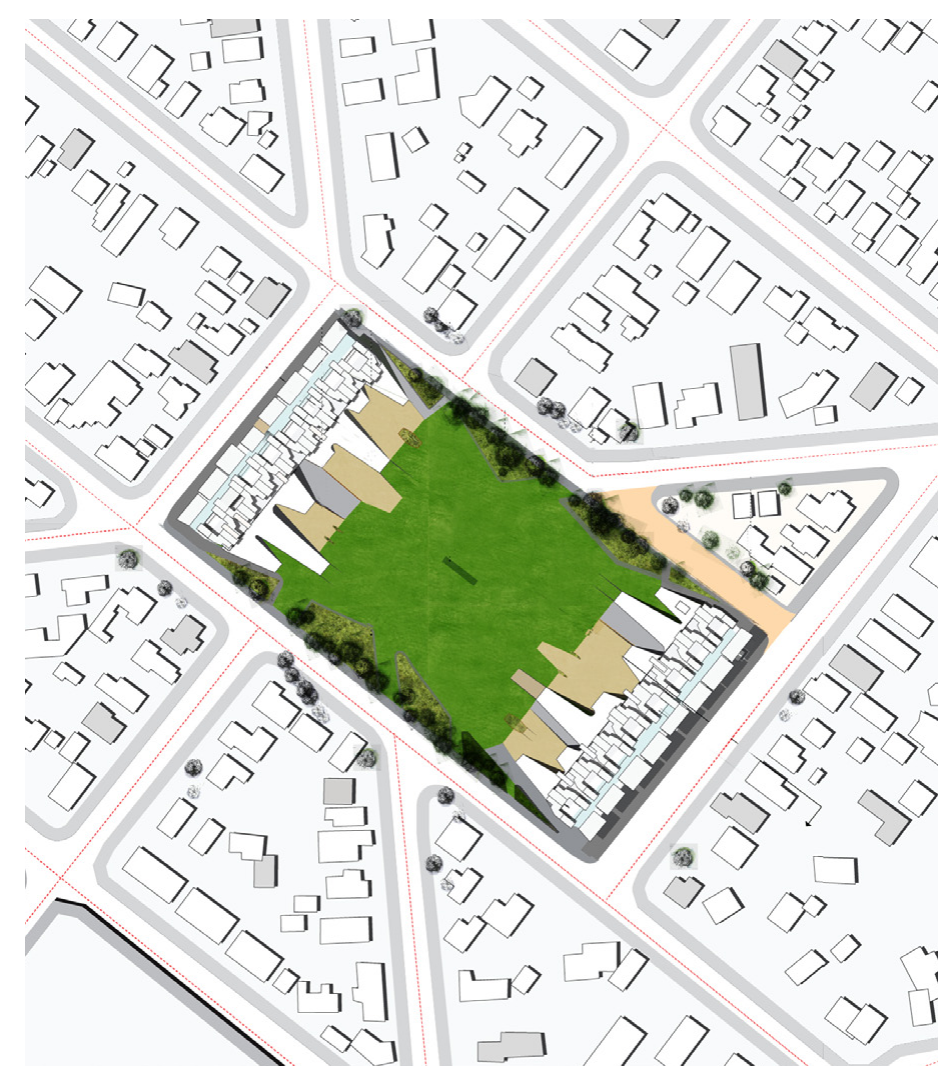

Figure 35 : A Forged Familiarity. Occupying the site as a deeply contextual and yet radical intervention - through its typological gesture injected with a fragmented; formal language and staggered street front - the project a fragmented; formal language and staggered street front - the project 


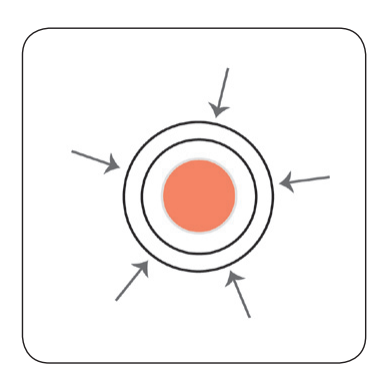

\section{A Resolute Fabric [constructing public space]}

By clearly expressing and articulating its constituent elements, the built form is afforded a certain confrontational, formal presence (and potency) in its context. This results especially from the interfacing of different types and architectural languages; the proximity of different programmes as formal and spatial expression; and the explorations into individual versus collective form. These strategies imbue the building with a state of compositional tension between its parts - as the upper mass is suspended over the lower and the smaller volumes puncture through the larger mass, for example.

At a more holistic scale, the 'separate' or 'resolute' nature of the architecture (as object) is somewhat down-played by its immediate setting - as the formal engagement with the park dissolves the building's 'limits'. Likewise, on the street side, the architecture operates more a resolute 'fabric' than object - with its constantly permeable, punctured and modulated facade. In these ways, the building opens itself up and engages its physical context, rather then asserting its separation from it. (see figure 37,38 )

Yet, while the building may not read as 'discrete' or separate 'object form' per se, neither does it dissolve passively into the space around it. Instead, it actively frames and gives form to the space it affronts negotiating public/private boundaries through its formal articulation. Even as the open space enters between the building's primary masses, the public/ private realms remain articulated as separate, as the interstitial space is clearly expressed as a void. (see figure 36 )

Pronouncing its formal nature in this way, the architecture is both separate and engaged; composed and casual; object and fabric; unified and agonistic with notions of 'difference' embodied and public space articulated. To this effect, the architecture forges and negotiates Sumner's emergence as an urban centre - not civic or economic, but necessarily 'public', and imbued with a certain socio-cultural potency.

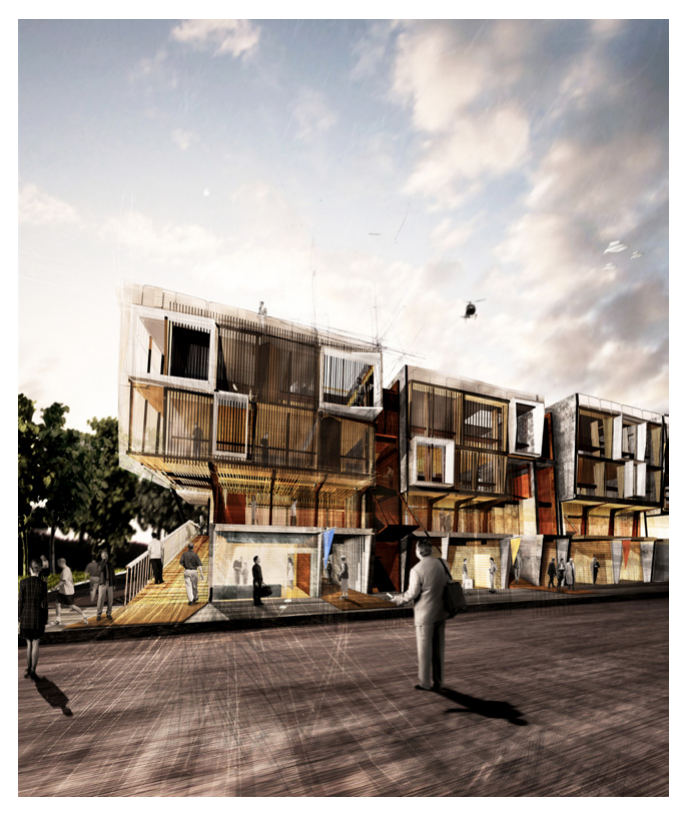

Figure 38 : A Resolute Fabric:. The buliding embodies ideas of seperation in and difference in its compositon: but as a whole it ference in its compositon: but as a whole it
works more as a resolute 'fabric' than object. Figure 36 : Negotiating Public Space. Through the seperation of
elements (at the building scale) and juxtaposition of rarefied central space with the dense built forms that bound it (at the site scale), the architecture frames and actively'constructs' public space. By letting this open space enter into the building, between the primary masses, ideas about the sub-centre as a public realm are provoked.

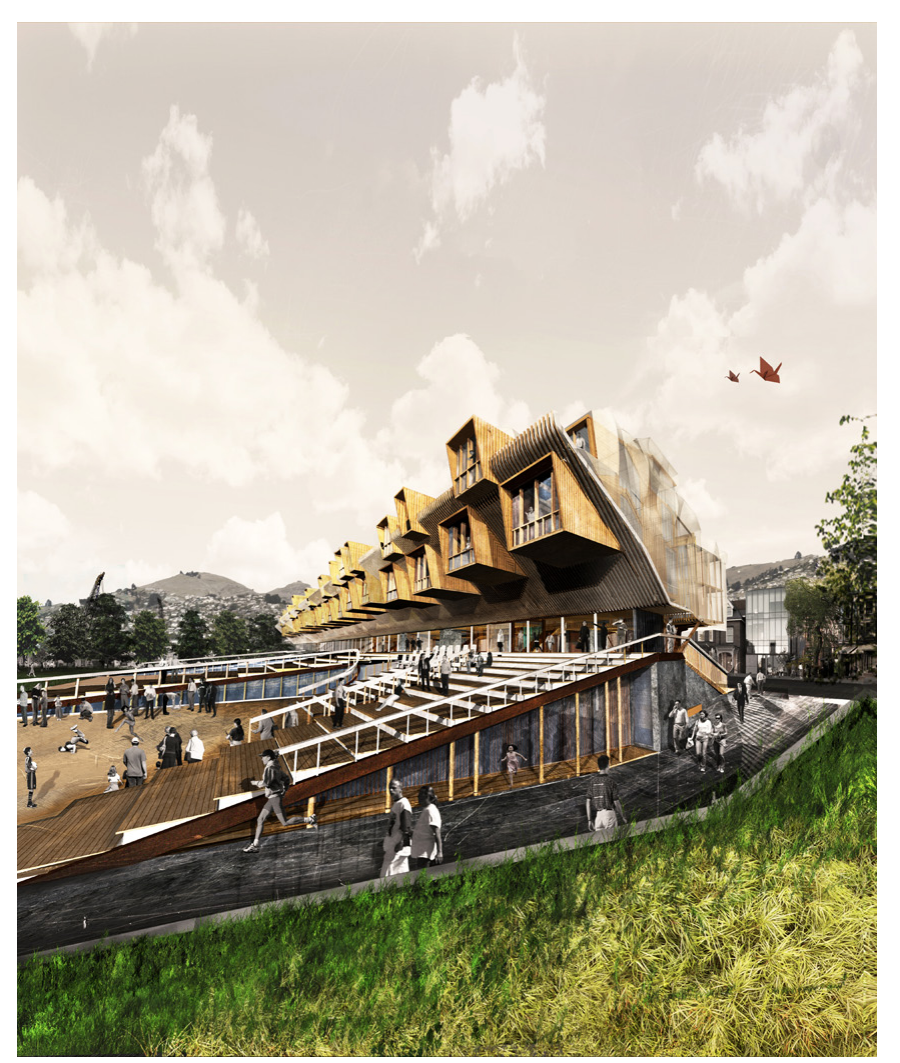

Figure 37 : An Embodied Tension. The compositional tension between elements (the upper and lower masses; the smaller units above, for example) imbues the building with a certain state of difference. Holistically, the building pronounces itself as a resolute and seperate 'object' in some ways (as it carves itself out of the ground plane, for example); yet is completely engaged with its phsyical context in others (as a contionous fabric). By articulating its form in this way - the architecture forges ideas of centrality; and a tension between public and private. 


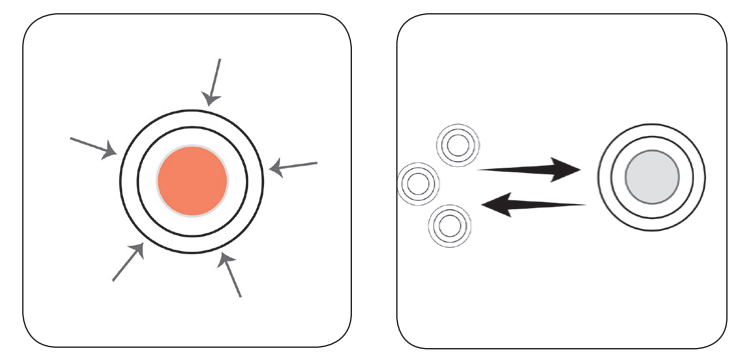

\section{An Exploitation of New Dialogues}

Though its formal and spatial configuration, the architecture exploits and engages new dialogues and tensions that may arise as the wider urban context transforms.

Between living and working, a relationship is forged that is not just functional but indeed spatial, formal and visual: embodied in the architectural response as the live-work chasm becomes a fundamental aspect. Relationships between public and private are tested and formalized through the separation of elements: where public space is physically compressed between the upper and lower masses; brought into dialogue new activities at it wraps up and through the building Likewise, new modes of habitation - densified and communal, individualized and collective - are extrapolated in the formal response. In this way, the pavilion gesture is formally reconfigured by its uncanny amalgamation with housing units. (see figures 39-41)

Engaging and extrapolating what these live-work dialogues; new modes of habitation; and new 'public' agendas might mean for this transforming urban context the architecture must be seen to depart from a 'form-follows-function' approach: rather the form somewhat deflects and directs these new functional, social, cultural opportunities: spatializing, formalizing and enacting them in a more operative and active manner.

In this way, a resonance is enabled between the form of the building and the social, cultural and programmatic context of the sub-centre more generally - as an urbanizing condition rife with new conditions.

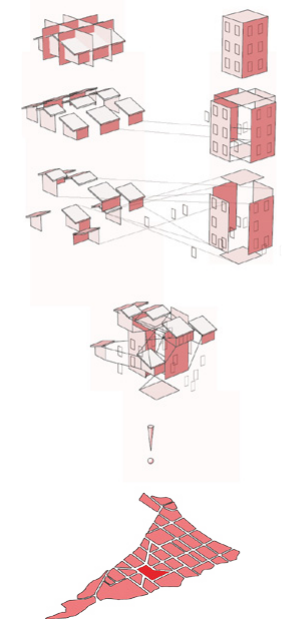

Figure 39 : Live-work as Architectural Potential. The bringing together of living and working cultures is exploited and extrapolated as an architectural gesture.

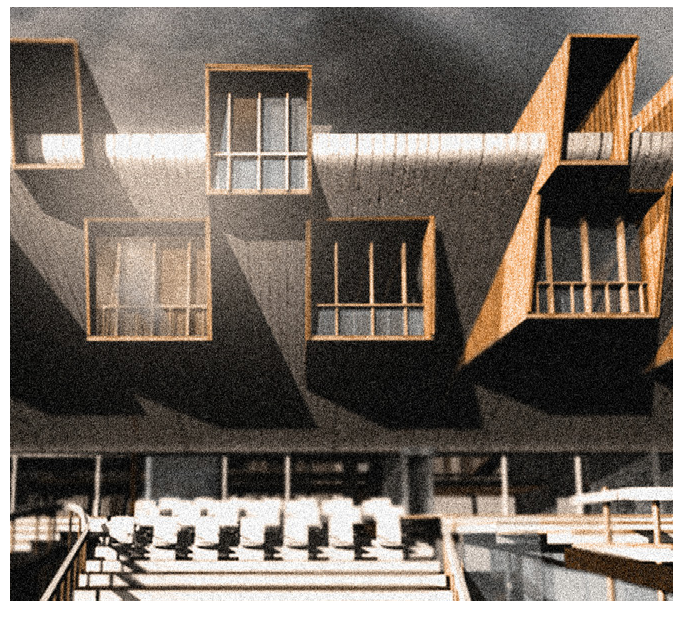

Figure 40 : Formalizing New Habitations. The amalgamation of housing units with the pavilion gesture foregrounds (enacts) ideas about new social and cultural dialogues.

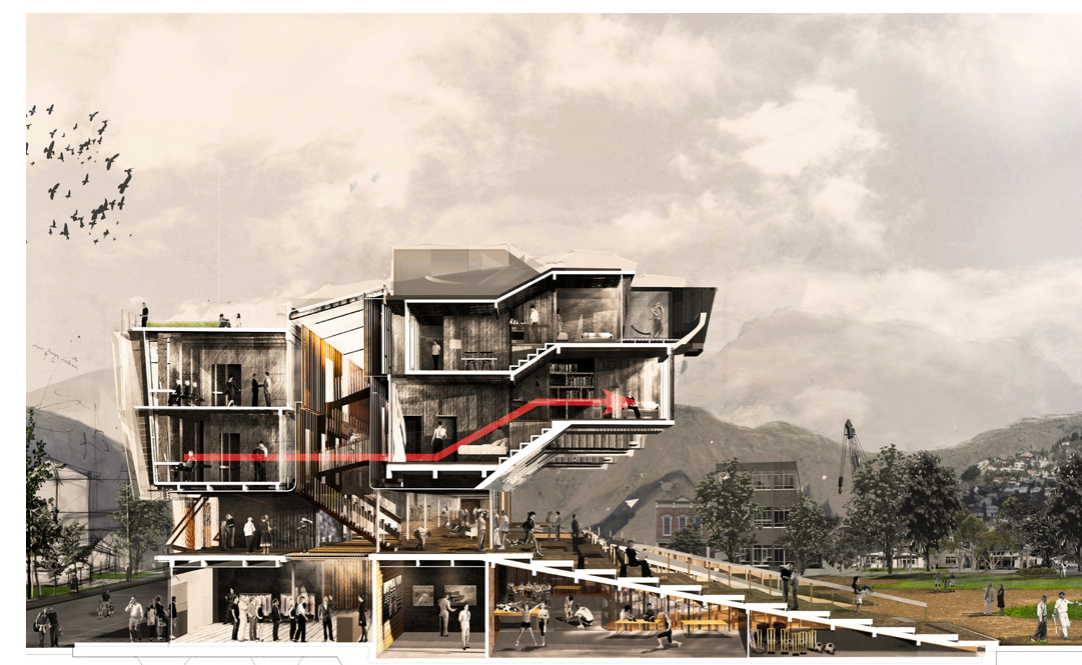

Figure 41 : Exploiting Live-work Dialogues. The dialogue and proximity of living and working is exploited and enacted in the formal and spatial gesture - though the organisation of spaces around a central live-work chasm. This is not as a case form follows function, but rather form deflecting, directing and catlysing new programmatic opportunities - enabling a resonance between the architecture and the sub-centre as a densifying urban environment. 

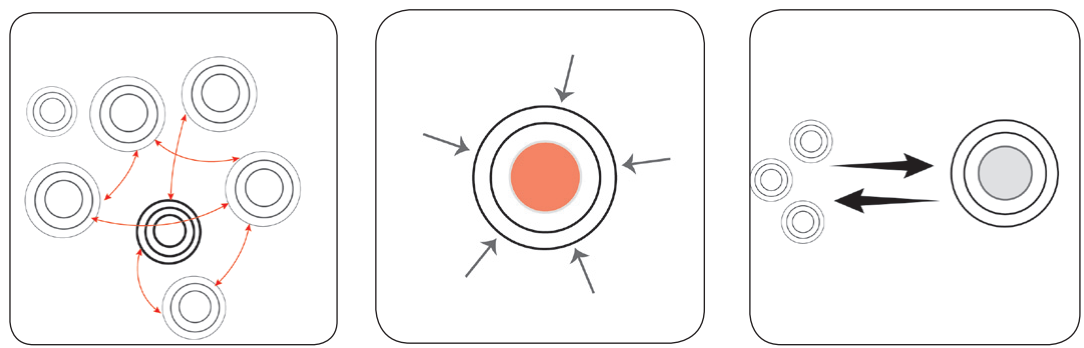

\section{A Fresh Face [an embodied opportunity]}

The intentional and experimental approach to architectural form delivered through the strategies above allows an architectural intervention that certifiably departs from the regular: in concept as much as form or aesthetic.

This is not to suggest the building becomes an 'icon' for Sumner, so much as that its imaginative and testing approach engages the opportunities of this transforming context somewhat holistically: exposing Sumner as unique urban context; harnessing what it might mean to be $a$ centre but not the centre, and presenting a fresh face to the urban(izing) context that may sponsor these opportunities.

This understanding of the project is somewhat intrinsic and ingrained in the previous discussions: the living and working spaces fundamentally in dialogue; the characterising transition between street and park; the tension between object and fabric; the notion of a housing pavilion; the reconfiguring of an endemic suburban site, the interfacing of different architectural types, and the unapologetic articulation of public space. Collectively, these impress upon the idea that this urban context both invites and requires new responses and engagements - in order to abet its necessary and effective transformation; and to catalyse its emergence as an identifiable place within a larger urban system. (see figure 42)

While this is of course a more general mode of relating to this urban context, it is perhaps also the most important manner in which the project may be seen to engage it.

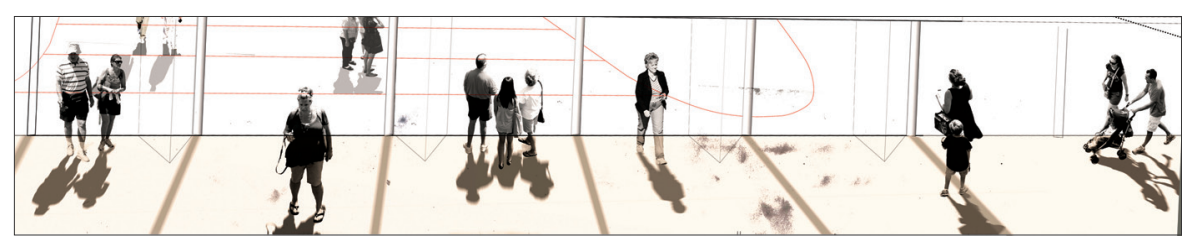

Figure 43: A Public Porch. The architecture activates new

notions of public (and private) space in the sub-centre through its formal experimentation.

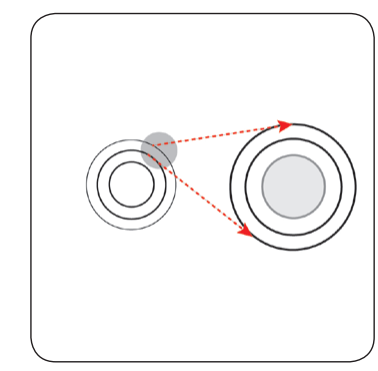

"What we're good at is the really weird stuff"

Sir Paul Callaghan, on the need to sponsor New Zealand's innovation economy (2011)

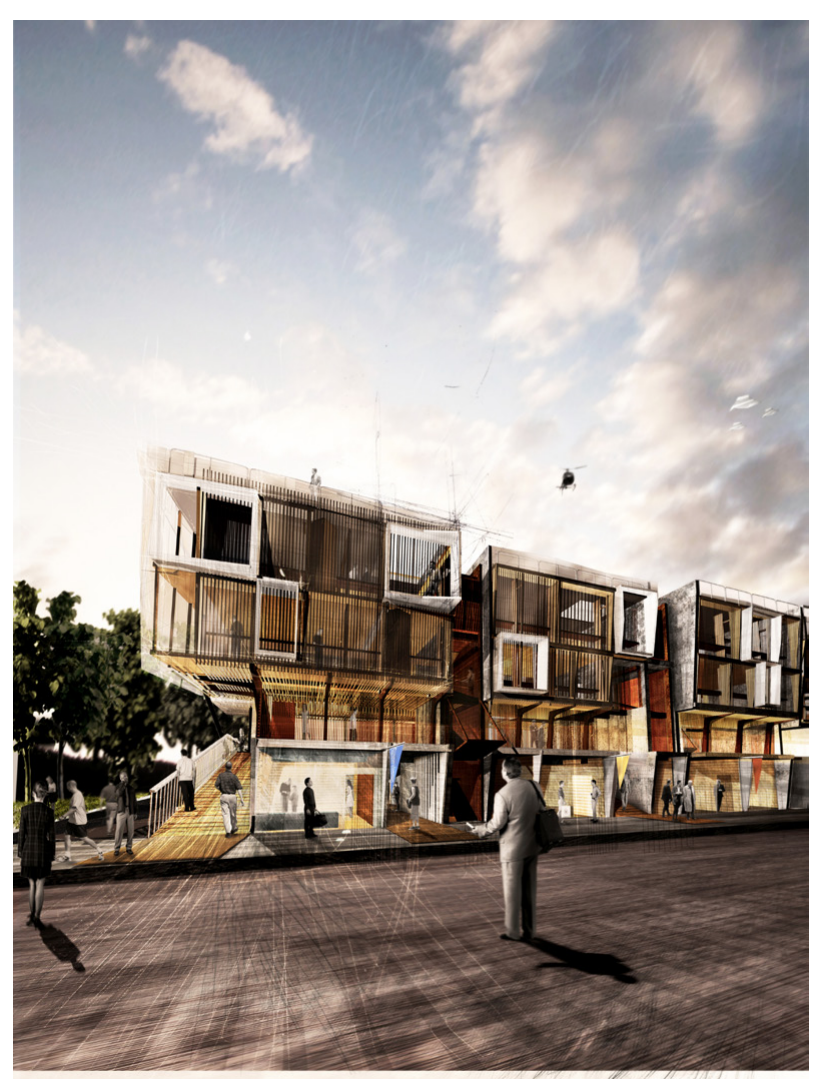

Figure 42 : A New Response. With its expressive and informed architectural tectonic, the project departs from a typical or normative response - attesting to the need for new opportunistic approaches, in order to catalyse the sub-centre's transition into a independent, social, cultural centre. 


\section{A Catylytic Intervention?}

Inasmuch as this design project was able to set its own constraints, its broadest objective - to respond explicitly to the emergent sub-centre - could be extensively explored, and was done so particularly through tectonic, visual and spatial means (here considered 'formal').

Having identified and understood these strategies retrospectively, it is clear that the project's immediate site had a lucid impact: indeed, the characteristic conditions of park and street may be seen to have significantly influenced the design outcome - in way that is to some degree only to be expected. Yet, somewhat more generally, the formal gestures and strategies employed may also be seen to transcend the immediate site conditions: the typological advance; the expression of density; the composition and separation of elements; the formal engagement with 'landscape'; the relationships between part and whole and the articulation of interstitial space, for example. Without suggesting the two are extricable, these latter gestures operate as much in relation to the wider urban conditions (cultural, social, programmatic) as the immediate site itself.

These formal strategies must be seen to have had some effect. Indeed, through its extensive exploration, the project has been able to engage not only its immediate site (St Leonard's Square), but to some degree, the emergent sub-centre forming its wider cultural, social and political context: relating to it as a densifying suburb; an urbanizing periphery; a space of transition and opportunity; a emergent public realm and a locus of new social and functional dialogues. As discussed on previous pages, these notions are variously reflected, embodied, enacted or attested to in a project that is as radical as it is contextual; as opportunistic as it is critical; and as exploitative as it is responsive.

This is not however to suggest that An Agora Anew is by an means a finite result or a perfect embodiment of the sub-centre (if such concept were indeed to exist): and a number of limitations of the project in relation to its objective may be duly pointed out here. Firstly, the essential 'freedom' of the design project may be considered its downfall. With further constraints and limitations the formal liberties would likely have been restrained somewhat more than they were - and the architectural response invariably inflected by other concerns. A more rigorous attentiveness to other'pragmatic' aspects such as program and scale, for example (rather then allowing them to be dictated ad lib) would have likely provided further challenges and opportunities against which to test a formal approach, and may have procured a more critical and informed outcome.

Secondly, the physical conditions of the site probably had a more salient influence of the final result than conceded here: the sub-conscious desire to relate materially and aesthetically to the existing street context, and indeed, the potent influence of 'the park' on the project (as discussed). While these contextual derivations may give the design its strength (or appropriateness), they may be seen to have comprised the potency of the design , as a project explicitly engaging it wider urban context (in a more embodied sense). In this way, it may be interesting to consider how those same strategies discussed would manifest on a different site: one less open and slightly more 'typical' - which may present further constraints, as well.

Thirdly, my own personal callings as a designer certainly had a vivid role to play in the design outcome - and I would not be so naive to suggest that it is 'objectively' related to its urban condition, by any means.

\section{A Project(ion) on the Sub-centre?}

Still, notwithstanding these limitations (or rather, inclusive of them), An Agora Anew - in both process and outcome - exposes more broadly the possibilities of an architectural response predicated on the emergent sub-centre as a specific urban condition. As it divulges these possibilities through a speculative, formal and site-specific response, the project presents but one example of what the sub-centre might mean for architecture; and conversely, what architecture might do in the sub-centre: Sumner, or otherwise.

To this end, conceivably, engaging the sub-centre need not be an ambition overlooked. Rather, by taking a position on the this urban context as much as it takes a position in it (and avoiding passivity on all counts), architecture may perhaps have a catalytic role to play within this transforming urban space, and as such, may enable Christchurch's peripheral suburbs to emerge as veritable urban centres, within a larger polycentric whole. 

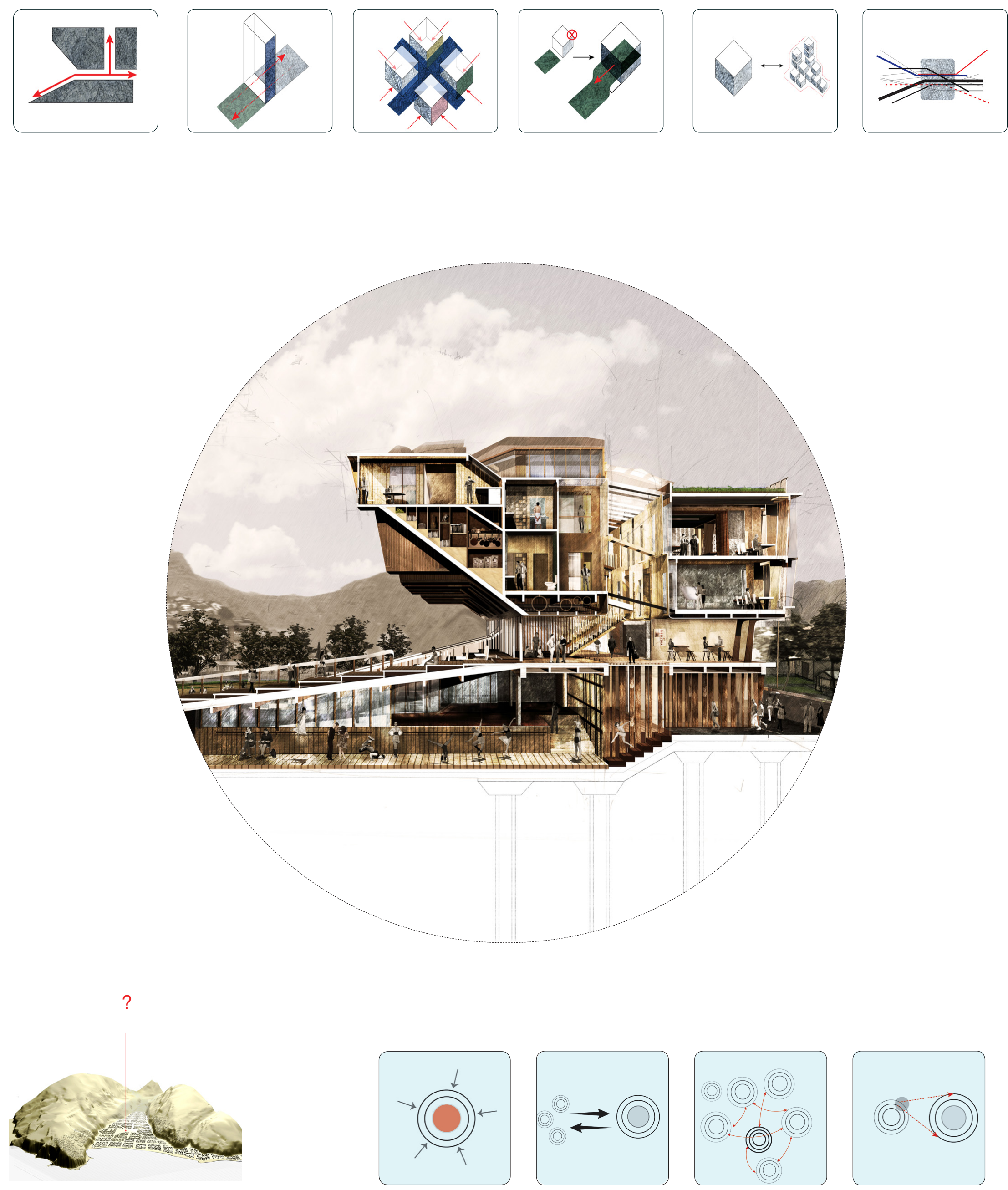


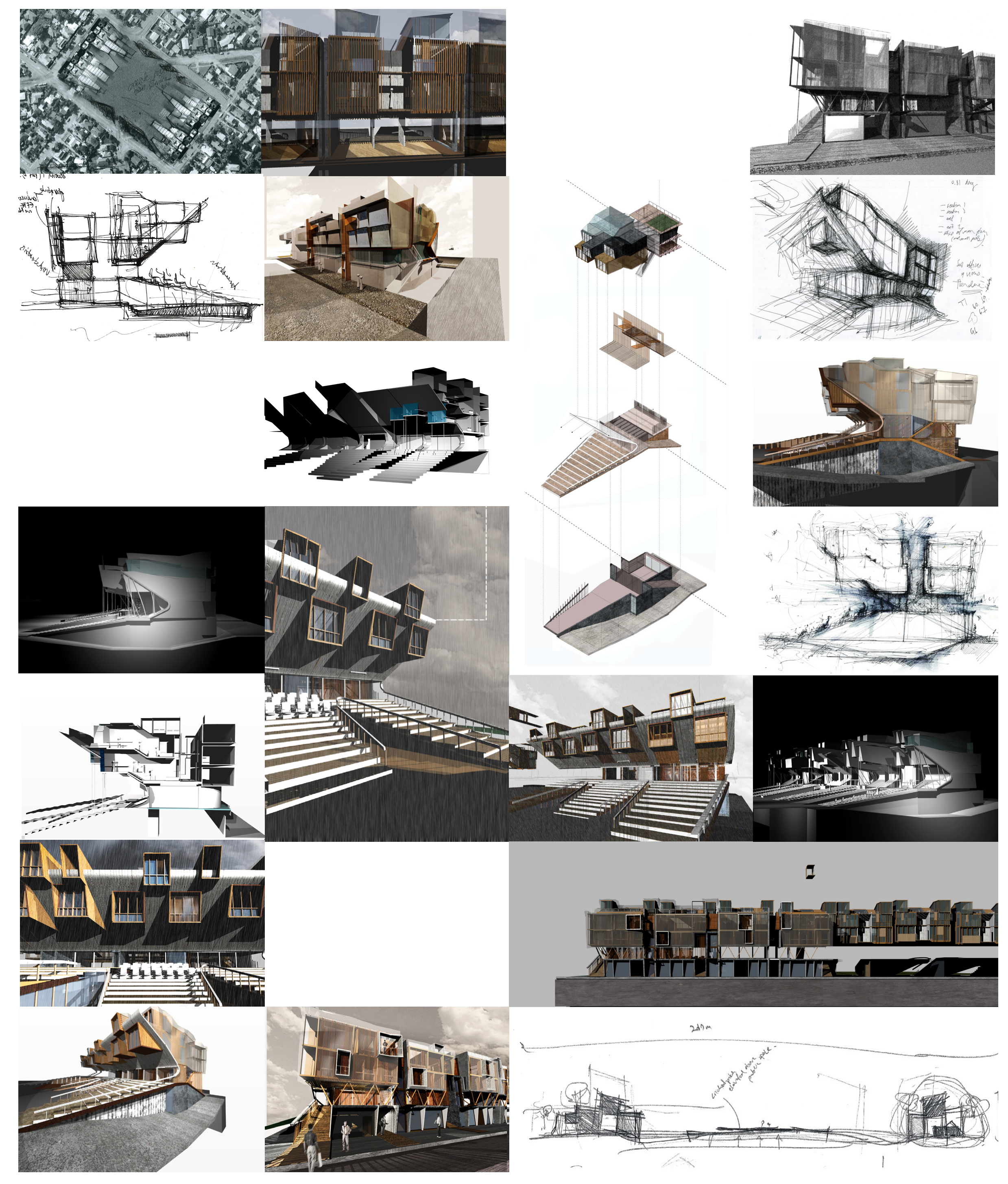



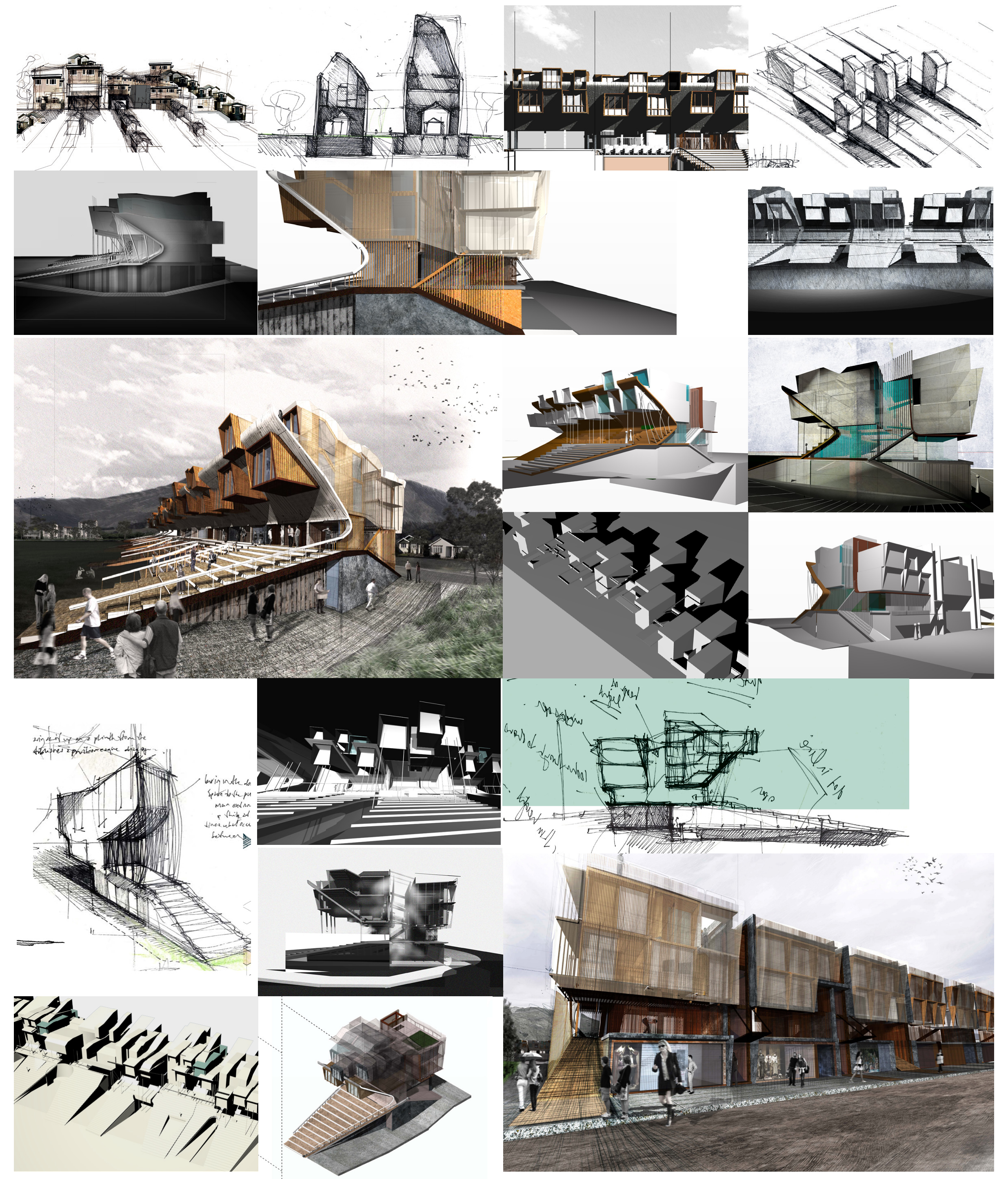

$\wedge$ Figure 45 : A Design Project on the Sub-centre:
A matrix of process images from throughout the design project. 


\section{Upon the Prospect of Suburban Lights: widening the gap for}

Toward a conclusion I will return to the initial provocation of this thesis: the paradoxical notion of suburban lights, in the wake of an earthquake-ridden Christchurch. The uncanny ring to this phrase raised multiple questions, which may be seen to broadly relate to two categories: firstly, about what Christchurch's latent polycentric condition (and its emerging sub-centres) might mean for architecture; and secondly, about what architecture might do in response. More broadly, these questions were underwritten by a larger one about architecture's urban operativity: simply, what can architecture do in the city?

\section{Looking Back: recounting a project for the sub-centre}

The design-led research methodology deployed in this thesis has been able to expand on each of these provocations in equal measure - and ultimately, in relation to one another. To the first; it has considered and framed, the emergent sub-centre as presenting both a need and an opportunity for architecture. To the second; it has divulged the possibilities for architecture to respond, and to enable its operative role within a sub-centre context.
The first section of this thesis, 'A Project for the Sub-centre', presented a site-specific speculative design located in Sumner Christchurch. The housing pavilion, live-work precinct, or new Agora, explored architecture's role in a specific emergent subcentre context: an exploration as evident in the conception of the project as the design output itself. Construed as a catalyst for Sumner's effective transformation into a veritable urban-centre, this project was fundamentally responsive to a Christchurch condition.

The second section, 'Towards the Sub-Centre as Architecture's Project', expanded on the research questions in a broader discursive context, employing a basic 'problem-opportunity' format. Firstly, it cast light on widely-observed problematic tendencies surrounding the generation of the urban sub-centre, and argued that architecture's urban instrumentality in this context must be actively addressed. Secondly, it negotiated a means through which architecture might directly engage the sub-centre as its 'project', discussing contemporary ideas around architecture's active relationship to the city, and eventually deploying these in a sub-centre context. Particularly, it argued that a formal approach described as critical-opportunistic or radical-resolute may allow architecture to operate as a critical sub-centre medium.

Significantly, the effective distinction between these two research components enabled the initial provocations to be doubly examined: the design project and discursive argument each useful in different ways; and indeed, each offering something the other could not. Particularly, where the design 
presented a specific response to the research questions, in relation to an holistic set of concerns (programme, structure, organization, site, context); the discursive argument enabled a more holistic exploration of something more specific: considering architectural form in relation to the idea of the subcentre.

Most importantly however: the latter (argument) allowed the former (design) to be interpreted and understood as a project for the sub-centre - as discussed in the exegesis. Given the limited ability to 'test' the design through alternate means, framing the project against the broader ideas developed in the paper allowed the successes and limitations of the design to be considered somewhat objectively: effectively enabling a critical reflection on a critical design.

\section{Looking Forward: towards two possible implications}

Considering each of these three sections - the design, the paper, and the exegesis - as a single unified finding, the implications of the design-research may be seen as twofold:

\section{[1] For the Sub-centre as a Necessary Project for Architecture}

The implications here are - quite plainly - for the architecture in the sub-centre at large. By firstly bringing attention to the clear need for architecture to actively engage this urban context; and secondly, identifying a means (and example) through which it might do so, the research can be seen to have broad implications: both in relation to a Christchurch context, where the need to address this imminent polycentric reality is transparent; as well as in relation to emergent sub-centres more generally - indeed, understood to bear similarities across a broader scale.

Specifically, the ideas about architecture's resistant-opportunistic engagement with the sub-centre through form, as well as those more specific ideas divulged in the exegesis, may find some use: deployed in a design context alongside other concerns, for example. Considering how these formal approaches may reveal themselves within projects for different sites (in Sumner); different sub-centre contexts; or different polycentric cities altogether, may raise some interesting questions in this regard - whether and how they could be translated, and to what degree? What opportunities could this present for design in such contexts - and, indeed, to what end for the sub-centre itself?

However, where this formal design approach (or ethos) may not be 'applied' in actuality per se (and indeed, was never intended to be 'prescriptive' so much as investigative), the argument that architecture should endeavor to actively address the subcentre (through whichever means it can) has a wider bearing. In other words: understanding the sub-centre as a distinct problem for architecture is as least as important as the specific mode of architectural response this may invite. (That said, however insofar as a 'critical' response is necessitated, a renewed formal consciousness is likely to be invoked, in any case).

Ultimately, the possibility that architecture may come to assume a more productive and catalytic role in relation to these emergent urban centres, has an irrefutable importance: not just for the subcentre itself, but for the connected, cohesive polycentric cities of which they are a part. Certainly this has a distinct relevance for Christchurch, given the fact that its CBD has only recently been opened up for public 'visitation' and is decades from 'completion'. Yet, given the tendency for this ubiquitous urban model to sport common characteristics (and problems), the possibility of a more productive architectural engagement with the sub-centre has a more transcendent significance.

Ultimately, the possibility that architecture may come to assume a more productive and catalytic role in relation to these emergent urban centres, has an irrefutable importance: not just for the sub-centre itself, but for the connected, cohesive polycentric cities of which they are a part.

On a final note regarding architecture in (or of) the sub-centre it has become evident that there is both scope and need to extend architectural discourse around this topic - indeed, given its indisputable presence as a $21^{\text {st }}$ century urban phenomenon. While here I have conducted but one line of research - and certainly not without its limitations - further and more diverse investigations into what this urban condition and architecture (as an urban medium) may mean for one another, are quite necessary. To this end, the design and discursive ideas explored in this thesis may serve as a platform for further research. 
[2] For Architecture in, of, and upon The City

Secondly, the implications of this thesis must be seen in relation to the wider question what can architecture do in the city? While this design-led thesis addresses a specific urban condition (the sub-centre), it nevertheless reveals the importance of thinking critically and directly about architecture's role in the city - the need to continually probe how architecture may act as an urban catalyst; and the opportunities that may arise for design, research, and indeed design-led research in this regard. This thesis inherently suggests that in order to truly gauge how architecture might operate on its urban contexts from the insideout, it is as important to ask why architecture? (to investigate the city as a distinct problem for architecture) as how architecture? (to consider modes of operation through which architecture may become engaged): and as to interrogate and test these possibilities within actual design contexts.

This thesis inherently suggests that in order to truly gauge how architecture might operate on its urban contexts from the inside-out, it is as important to ask why architecture? [...] as how architecture? [...] as to interrogate and test these possibilities within actual design contexts.

In a century where urban contexts are undergoing morphological and conceptual change in a virulent measure, engaging these types of questions in both design and discourse is of unparalleled importance - not just for architecture to prove that it still means something in the city; but moreover, for the city itself as the locus of social, cultural, political, economic and public activity.

It seems then that a more appropriate question for a discipline equally critical and creative - within urban contexts requiring and eliciting those actions in equal measure - is not what can architecture do? but what can it not? 


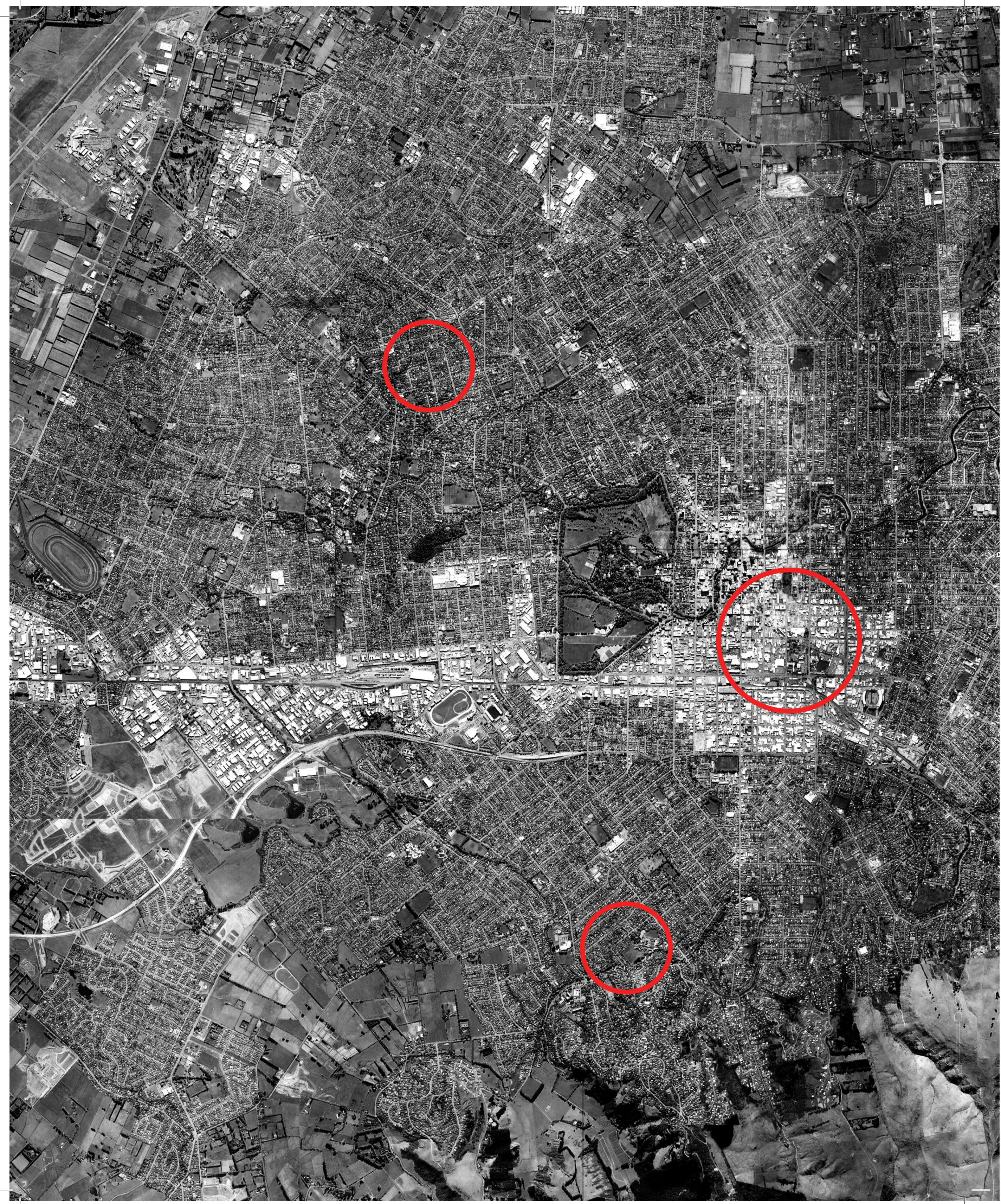




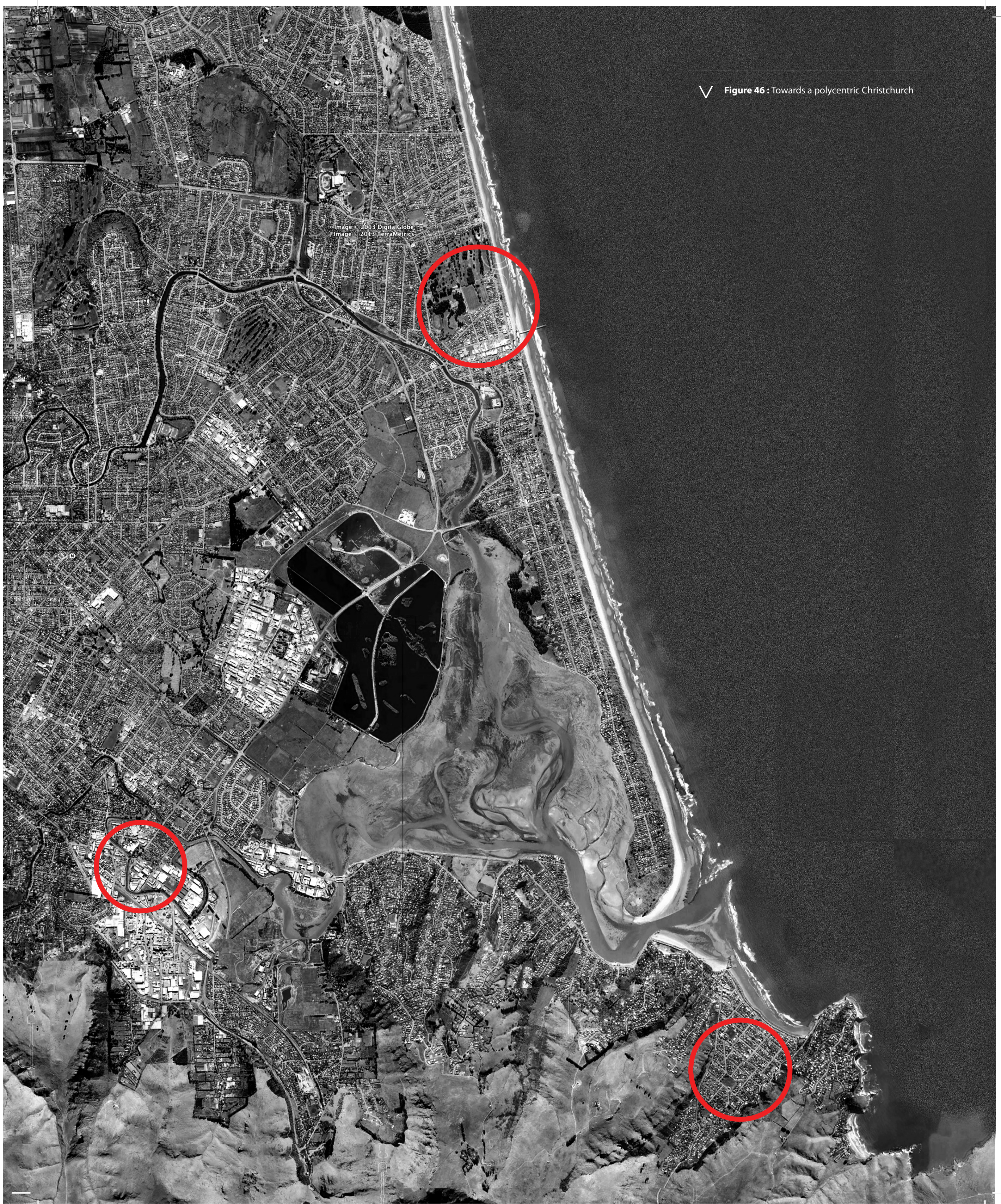


"It's all about the Discipline"

- A. Earl \& G. Mills, 2013. 


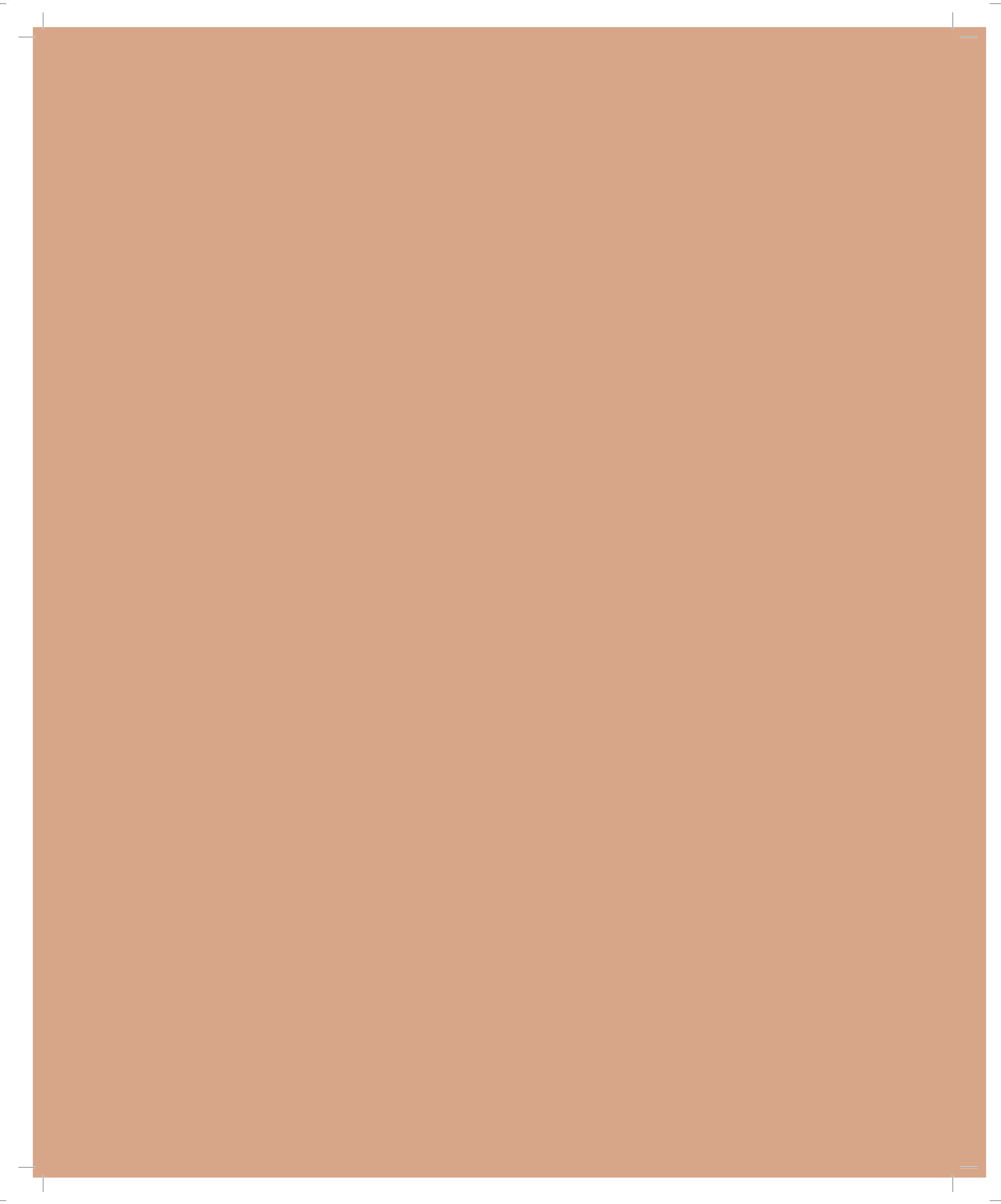




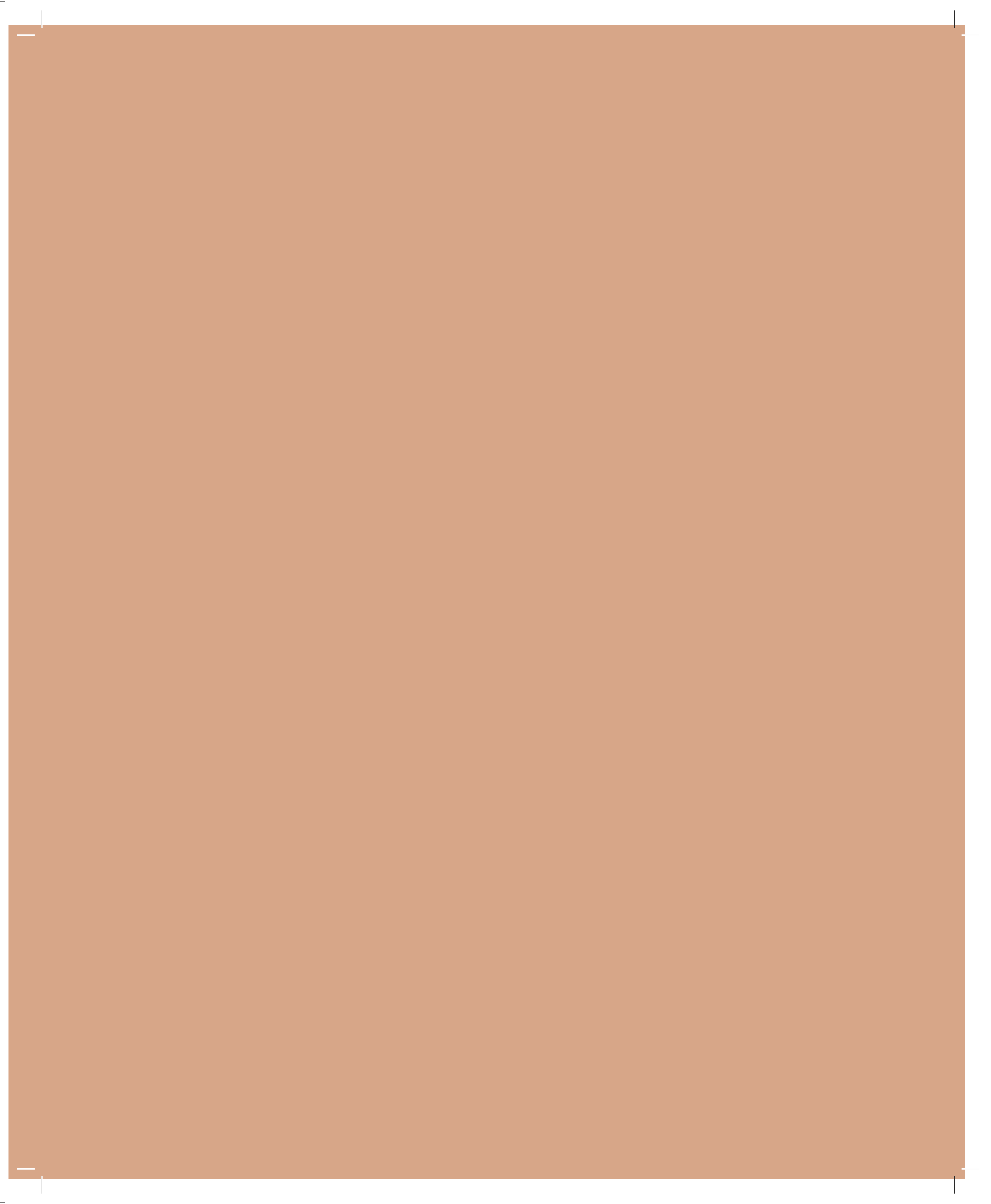




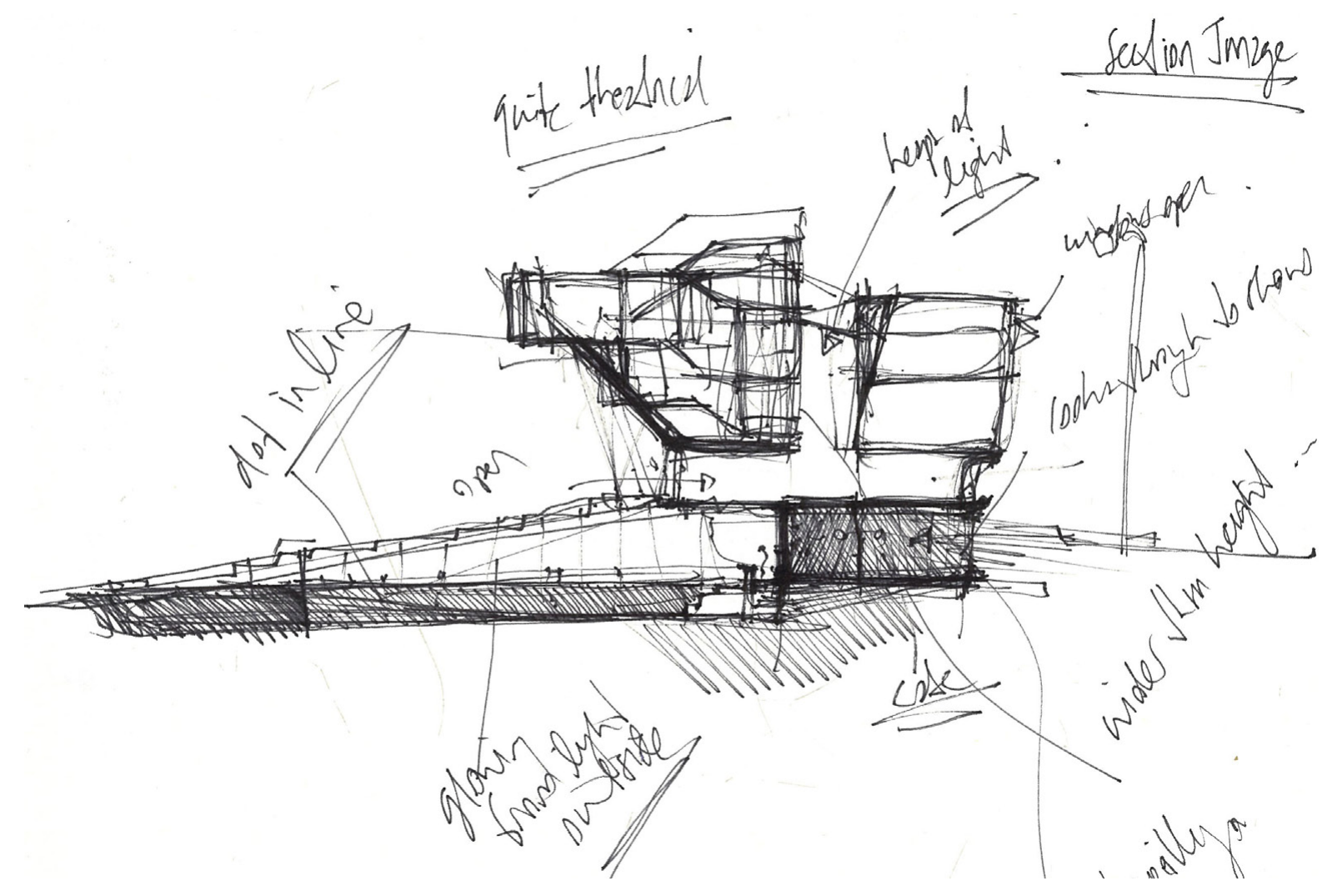

$\wedge \quad$ Figure 47 : Sectional sketch. 


\section{Bibliography}

Abrahamson, Michael. Book Review: The Possibility of An Absolute Architecture. 6 June 2011. http:// criticundertheinfluence.wordpress.com/2011/06/06/ book-review-the-possibility-of-an-absolutearchitecture/ (accessed February 20, 2013).

Actar. Total Housing: Alternatives to Urban Sprawl. Barcelona: Actar, 2010

Arup. "Chongqing Xiyong integrated planning." Arup website. 30 April 2012. www.arup.com/Home/Projects/Integrated_ planning_Chongqing_Xiyong_SubCentre.aspx.

Aureli, Pier Vittorio. "City as Political Form: Four Archetypes of Urban Transformation." Architectural Design: Special Issue: Typological Urbanism, January/Februrary 2011: 32-37.

-. The Possibility of an Absolute Architecture. Cambridge: MIT Press, 2011.

-. The Project of Autonomy. New York: Princeton Architecture Press, 2008

- "The Theology of Tabula Rasa: Walter Benjamin And Architecture in the Age of Precarity." Log 27, 2013: 10-15.

Bergdoll, Barry, and Reinhold Martin. Foreclosed: Rehousing the American Dream. New York: Museum of Modern Art, 2012

Berschaffel, Bart. "Art in (and of) Architecture: Autonomy and Medium." In The Sage Handbook of Architecture Theory, by C.Greig Crysler, Stephen Cairns and Hilde (Eds) Heynen, 165-176. London: Sage Publications Ltd, 2012.
Blau, Eve. "Reinventing Practice: Architecture and the City After Socialism." In A Peripheral Moment: Experiments in Architectural Agency, Croatia 1999-2010, by Ivan Rupnik, 4-5. Barcelona: Actar, 2010.

Bolling, Lars. "Creating "Spatial Identity": A way to improve the periphery?" In City Images and Urban Regeneration, by Frank Eckardt and Peter (eds.) Kreisl, 225-243. Frankfurt: Peter Lang Publishing, 2004.

Brook, Kip. “Christchurch CBD needs rethink." The Big Idea. 25 April 2011. http://www.thebigidea.co.nz/news/ industry-news/2011/apr/85081-christchurch-cbdneeds-rethink.

Brugmans, George, and Jan Willem (Eds.) Petersen. Making City: 5th LABR 2012. Rotterdam: International Architecture Biennale Rotterdam, 2012.

Bureau Stedelijke Planning: General Information. 15 September 2012. http://www.stedplan.nl/ (accessed January 28, 2013).

Canterbury Earthquake Recovery Authority. The Recovery Plan 30 July 2012. http://ccdu.govt.nz/the-plan (accessed June 15, 2013).

Castle, Helen. “Editorial.” Architectural Design: City Catylst, 2012: 5.

Chung, Judy Chuihua, Jeffrey Inaba, Rem Koolhaas, and Sze Tsung Leong. Great Leap Forward / Harvard Design School Project on the City. Cambridge, MA: Taschen, 2002. 
Cladera, Josep Roca, Carlos R. Marmolego Duarte, and Montserrat Moix. "Urban Structure and Polycentrism: Towards a Redefition of the Sub-centre Concept." Urban Studies Journal, 2009: 2841-2868.

Cormier, Brendan. "The Cult of the Centre." Volume \#32: Centres Adrift, 2012: 4-7.

Cox, Katharine. "Grandstand: 89 Alfredton Road, EKETAHUNA." New Zealand Historic Places Trust Web site. 15 December 2010. http://www.historic.org.nz/ TheRegister/RegisterSearch/RegisterResults. aspx?RID=3971\&m=advanced (accessed July 17, 2012).

Cuff, Dana, and Roger Sherman. "Introduction." In Fast-Forward Urbanism: Re-thinking Architecture's Engagement with the City, by Dana Cuff and Roger (Eds) Sherman, 11-33. New York: Princeton Architectural Press, 2011.

Cupers, Kenny, and Isabelle Doucet. "Agency in Architecture: Reframing Criticality in Theory and Practice." Footprint: Delft School of Design Journal 4 (2009): 1-6.

Curtis, Carey, John L Renne, and Luca Bertolini. Transit Oriented Development: Making it happen. Surrey: Ashgate Publishing, 2009

Deamer, Peggy. Review of K.Michael Hays, Architecture's Desire: Reading the Late Avant-Garde and Pier Vittorio Aureli, The Possibility of an Absolute Architecture. Yale, n.d.

Dear, Michael. "Cities without Centers and Edges." In FastForward Urbanism: Rethinking Architecture's Engagement with the City, by Dana Cuff and Roger (Eds.) Sherman, 226-241. New York: Princeton Architectural Press, 2011.

Dehaene, Michiel, and Lieven (eds) De Cauter. Heterotopia and the City: Public space in a postcivil societyl. New York: Routledge, 2008

Diamanti, Jeff. "Architectural Positions." Reviews in Culture. 3 January 2012. http://www.reviewsinculture.com/?r=76.

Dictionary.com. Agora. 20 May 2013. http://dictionary.reference. com/browse/agora.

Easterling, Keller. "Active Forms." In Fast-Forward Urbanism: Rethinking Architecture's Engagement with the City, by Dana Cuff and Roger (Eds.) Sherman, 210-225. New York: Princeton Architectural Press, 2011.

Easterling, Keller. "We Will Be Making Active Form." Architectural Design, 2012: 58-63.
Eckardt, Frank, and Peter Kreisl. “City Images and Urban Regeneration (Introduction)." In City Images and Urban Regeneration, by Frank Eckardt and Peter (eds.) Kreisl, 13-22. Frankfurt: Peter Lang, 2004.

Eisenman, Peter, interview by Carlos Brillembourg. Peter Eisenman Interview Bombsite, (Fall 2011).

Eisenschmidt, Alexander. "Stranger Than Fiction: A Mission Statement." Architectural Design: City Catylst, no. 5 (2012): 14-17.

Eisenschmidt, Alexander. "The City's Architectural Project: from Formless City to Forms of Architecture." Architectural Design 5 (September/October 2012): 18-25.

Fischer, Ole W. "Architecture, Capitalism and Criticality." In The SAGE Handbook of Architectural Theory, by C. Greig Crysler, Stephen Cairns and Hilde (eds) Heynen, 56-69. London: Sage Publications Ltd, 2012.

Frei, Hans. "What is Architecture?" El Croquis, 2009: 20-27.

Gyurkovich, Mateus. "In Search of the Urban Composition of Sub-Centres in Polycentric European Metropolises." ACE: Architecture, City and Environment, 18 February 2011: 251-264.

Hall, Peter, and Kathy Pain. The Polycentric Metropolis: learning from mega-city regions in Europe. London: Earthscan, 2006.

Hays, Michael K. Architecture's Desire. New York: Princeton Architecture Press. 2010

Hays, Michael K. “Critical Architecture: Between Culture and Form." Perspecta 21 Fall (1984): 15-28.

Hilberseimer, Ludwig. Metropolisarchitecture and Selected Essays. New York: GSAPP Books, 2013.

Hinson, Mary Alice Dixon. "Forethoughts." In Type and the (Im) Possiblities of Convention: Midgard Monograph 2, by Garth (Ed.) Rockcastle, 5-6. New York: University of Minnesota College of Architecture and Landscape Architecture, 1991.

Hoare, Charlotte, and Matthew Webby. "Taking the City to the Suburbs." Architecture New Zealand, February 2012: 31-33. 
Houston, Alecia. The Co-operative Spirit: Bridging Social Captial in Mixed-income Housing Development Through Resident Empowerment, Invovement and Cooperation. Virginia, Virginia, 10 May 2012

Hutton, Thomas A. "Trajectories of the New Economy: Regeneration and Dislocation in the Inner City." Sagepub Web site. 16 April 2009. http://www.usj. sagepub.com/content/46/5-6/987 (accessed May 15, 2012).

Jacobs, Jane. The Death and Life of Great American Cities. New York: Random House, 1969.

Jameson, Fredric. The Cultural Turn: Selected Writings on the Postmodern, 1983-1998. London: Verso, 1998.

Jaume, Masip Tresserra. Polycentrism and emerging sub-centres in the restructuring of metropolitan systems. The Case of Barcelona Metropolitan Region (RMB). Miami, Miami, 9-12 November 2011.

Jaume, Masip Tresserra. Towards a Methodology to Identify and Characterize Urban Sub-centres: Employment Entropy Information versus Employment Density. Barcelona, Catalonia, 16 May 2012.

Kaji-O'Grady, Sandra. "Formalism and Forms of Practice." In The Sage Handbook of Architectural Theory, by C.Greg Crysler, Stephen Cairns and Hilde (Eds) Heynen, 152164. London: Sage Publications, 2012.

Kloosterman, R.C. and S Musterd. "The Polycentric Urban Region: Towards a Research Agenda." Urban Studies, 2001: 623633.

Koolhaas, Rem. Delirious New York: A Retroactive Manifesto for Manhattan. Reprint. New York: Monacelli Press, 1997.

Koolhaas, Rem, Bruce Mau, and Hans Werlemenn. S,M,L,XL. New York: Monacelli Press, 2002.

Krauss, Rosalind. “Death of a Hermeneutic Phantom: materialization of the sign in the work of Peter Eisenmann." In The Light Construction Reader, by Todd (Ed.) Gannon, 157-170. New York: The Monacelli Press, 2002.

Lange, Alexandra. Writing About Architecture: Mastering the language of buildings of cities. New York: Princeton Architectural Press, 2012.
Leach, Neil. "C<amo> uflage." In Considering Rem Hoolhaas and the Office for the Metropolitan Architecture: what is $O M A$, by Veronique Patteeuw, 89-100. Rotterdam: NAi Publishers, 2003.

Lee, Christopher C.M. "The City as a Project." The City as a Project. 16 August 2011. http://thecityasaproject.org/2011/08/ type/.

Lee, Christopher CM, and Sam Jacoby. "Typological Urbanism and the Idea of the City." Architectural Design, January/ February 2011: 14-23.

Lefaivre, Liane, and Alexander Tzonis. "The Question of Autonomy and Architecture." Tzonis. Unknown Unknown 1984. http://tzonis.com/dks/dks/ publications/online\%20publications/1984-HAR-The\%20 Question\%20of\%20Autonomy.htm (accessed March 23, 2013)

Lehmann, Steffen. “Lessons from Europe for China: New Urban Sub-Centres for a Polycentric Network City." Journal of Green Building 5, no. 1 (2010): 88-100.

Lehmann, Steffen. "Low-to-no carbo city: Lessons from western urban projects for the rapid transformation of Shanghai." Habitat International 37 (2013): 61-69.

Lindsay, Grehg. "Port-au-Prince 2.0: A City of Urban Villages?" Fast Company. 26 January 2011. http://www.fastcompany. com/1720799/port-au-prince-20-city-urban-villages (accessed December 12, 2012).

Lucas, Di. Un-Doctored. 21 April 2011. http://www. nzdoctor.co.nz/un-doctored/2011/april-2011/21/ christchurch\%E2\%80\%99s-cbd-should-not-be-fullyrebuilt-to-what-it-was-\%E2\%80\%93-landscapearchitect-expert.aspx (accessed May 20, 2013).

Lynch, Kevin. The Image of the City. Massachusetts: MIT Press, 1960.

Lyster, Clare, and Judith K De Jong. "Housing Urbanism." Journal of Architectural Education 65, no. 1 (2011): 7-12

Macarthur, John, and Naomi Stead. “Introduction: Architecture and Aesthetics." In The Sage Handbook of Architectural Theory, by C.Greig Crysler, Stephen Cairns and Hilde (Eds) Heynen, 123-1365. London: Sage Publications Ltd, 2012 
Manhire, Toby. "Darkness at the heart of Christchurch, one year after the quake." The Guardian Website. 19 February 2012. http://www.guardian.co.uk/world/2012/feb/19/ christchurch-earthquake-one-year-on.

Martin, Louis. "Frederic Jameson and critical architecture." In Political Unconscious of Architecture, by Louis Martin. Surrey, England: Ashgate, 2011.

Martin, Reinhold. "Critical of What? Toward a Utopian Realism." In Constructing a New Agenda: Architectural Theory 1993-2009, by Krist Sykes, 348-361. New York: Princeton Architectural Press, 2010 [2005].

McGrath, B., \& Shane, G. “Introduction: Metropolis, megapolis and metacity." In The SAGE handbook of Architectural Theory, by S. Cairns, \& H, Heynen (Eds.) C. Crysler, 641657. London: SAGE Publications, 2012

Muñiz, Ivan, Miquel Àngel Garcia-López, and Anna Galindo. "The Effect of Employment Sub-centres on Population Density in Barcelona." Urban Studies 45, no. 3 (2008): 627-649.

Myerson, Jeremy, Jo-Anne Bichard, and Alma Erlich. New Demographics New Workspace. Surrey: Gower Publishing Limited, 2010.

Oosterman, Arjen. "Adrift." Volume 32, 2012: 2-3.

Ostwald, Michael J. "Ranciere and the Metapolitical Framing of Architecture: Reconstructing Brodsky and Utkin's Voyage." Interstices, 2008: 9-20.

Rajchman, John. "Introduction." In The Pragmatist Imagination, by John (Ed.) Ockman, 114-115. New York: Princeton Architecture Press, 2000.

Reiser, Jesse. Atlas of Novel Tectonics/Reiser + Umemoto. New York: Princeton Architectural Press, 2005.

Rice, Charles. "Critical Post-Critical." In Critical Architectre, by Jane Rendell, Jonathan Hill, Mark Dorrian and Murray Fraser, 261-268. London: Routledge, 2007.

Ring, Trudy, Robert Salkin, and Sharon La Boda. International Dictionary of Historic Places: Southern Europe. Routledge, 1996.
Robinson, Julia W. “Premise, Premises: Architecture as Cultural Medium." In Type and the (Im)Possibilities of Convention: Midgard Monograph 2, by Garth Rockcastle (Ed.), edited by Garth Rockcastle, 157-164. New York: University of Minnesota College of Architecture and Landscape Architecture, 1991.

Rockcastle, Garth. "The Value of Type: A Debate." In Type and the (Im)Possibilities of Convention: Midgard Monograph 2, by Garth (Ed) Rockcastle, 7-10. New York: University of Minnesota College of Architecture and Landscape Architecture 1991.

Rossi, Aldo. Architecture and the City. 8. Edited by Aldo Rossi and Peter Eisenman. Translated by Diane Ghirardo. Cambridge, Mass: The MIT Press, 1982.

Rupnik, Ivan. A Peripheral Moment: Experiments in Architectural Agency. Barcelona: Actar, 2010.

Somol, R.E. "Shape and the City." Architectural Design: City Catylst, 2012: 108-113.

Tafuri, Manfredo. “'A'Architecture dans le Boudoir, The Language of Criticism and the Criticism of Language'." Oppositions, 3, 1974: 37-62.

Thomas Dolan Architecture. Live-Work. 15 May 2012. http://livework.com (accessed May 15, 2012).

Thun, Geoffry, Kathy Velikov, and Colin Ripley. “Re-Centering the Periphery." Volume: Centers Adrift (Archis) 32 (2012): 30-37.

Toorn, Roemer van. "Aesthetics as a Form of Politics." roemervantoorn. 2005. http://www.roemervantoorn. nl/Resources/Toorn,\%20Van_Aesthetics\%20as\%20 a\%20Form\%20of\%20Politics.pdf (accessed February 14, 2013).

Trummer, Peter. "The City as an Object:Thoughts on the Form of the City." $\log 27,2013:$ 51-57.

Tschumi, Bernard. Event-Cities. Cambridge, MA: MIT Press, 1994.

Tschumi, Bernard, interview by Alexander Eisenschmidt. Importing the City into Architecture: An Interview with Bernard SchumiEdited by Alexander Eisenschmidt. Architectural Design, (Unknown September 2012): 130-135. 
Tung, Greg, and Ellen Greenberg. "The Polycentric City." Places 17, no. 2 (2005): 81-83.

Ungers, Oswald Mathias. Die Stadt in der Stadt: Berlin das Grune Stadtarchiepel. Berlin and Cologne: Studioverlag fur Architektur, 1977

Vasanen, Antti. “Functional Polycentricity: Examining Metropolitan Spatial Structure through the Connectivity of Urban Sub-centres." Urban Studies (Sage) 49, no. 16 (2012): 3627-3644.

Vidler, Anthony. "The Third Typology." In Oppositions Reader: Selected Essays 1973-1984, by Michael (Ed.) Hays, edited by 1, 13-16. New York: Princeton Architectural Press, 1998.

Waldheim, Charles. The Landscape Urbanism Reader. Princeton: Princeton Architectural Press, 2006.

Walsh, Kelvin. "(re)Presenting Suburbia: image and identity at the city's edge." WALK 21 - VI Everyday Walking Culture. Zurich, 2005. 1-7.

Wigley, Mark. Constant's New Babylon: The Hyper-Architecture of Desire. Rotterdam 0: 010 Publishers, 1998.

Witte, Ron. "The Perfect Storm: Urbanism and Architecture." Edited by Alexander Eisenschmidt. Architectural Design, September 2012: 74-79. 


\section{List of figures}

\section{Introductory Section and Part One: A Project for a Sub-Centre}

Figure 1: Christchurch CBD after 2011 Earthquake with highlights. Author's own image.

Figure 2: Christchurch Earthquake Aerial Photograph. Source: kete.digitalnz.org. Web. 19 May 2013.

Figure 3: Christchurch CBD Photograph. Source: "65 Dead in Devastating Christchurch Quake". Stuff. Web. 21 April 2013.

Figure 4: Photograph of Damaged Christchurch Street. Source: "Massive earthquake strikes Christchurch", Yahoo News. Web. 27 January 2012.

Figure 5: Parti Diagram. Author's own image.

Figure 6: Diagram of Thesis Questions. Author's own image.

Figure 7: Diagram of Thesis Structure. Author's own image.

Figure 8: Render of porch (title page). Author's own image.

Figure 9: Matrix of Sketches. Author's own image.

Figure 10: Destructed CBD. Source: www.stuff.co.nz, accessed 10 April 2013.

Figure 11: Diagram of Decentralized and Polycentric Urban Structures. Author's own image.
Figure 12: Christchurch changing urban structure. Adapted by author from Architecture New Zealand, January 2012. p 31.

Figure 13: Wider Christchurch region showing CBD and Sumner (Aerial Photograph). Adapted from: Map, Koordinates. Web. 20 February 2013.

Figure 14:"A group of young bathers in bathing costumes in the surf at Sumner beach on the Anniversary Day of Christchurch [16 Dec. 1918.]". Photographer Unknown. Source: Christchurch City Libraries Heritage

Figure 15: Comparison of Sumner and Wellington. Author's own image.

Figure 16: Aerial photograph of Sumner. Adapted from: Map, Koordinates. Web. 20 February 2013.

Figure 17: Sumner main road lined with containers. Photograph by author.

Figure 18: Giant boulders and landslides in Sumner. Photograph by Hannah Johnson. Source: "Earthquake in New Zealand", The Atlantic. Web. 25 March 2013.

Figure 19: House hanging off cliff in Sumner. Photograph by author

Figure 20: St Leonard's Square Aerial Photograph. Adapted from: Map, Koordinates. Web. 20 February 2013.

Figure 21: Cricket Pitch. Author's own image. 
Figure 22: Model of Sumner showing site and town village. Author's own image.

Figure 23: Site analysis. Author's own image.

Figure 24: Photograph of Typical Sumner Street. Photograph by author.

Figure 25: Composite image of threefold context of design. Author's own image.

Figure 26: Longitudinal section. Author's own image.

Figure 27: Distribution of built form. Author's own image.

Flgure 28: A two-sided gesture. Author's own image.

Figure 29: Integration into street network. Author's own image.

Figure 30: Street-park condition addressed by built form. Author's own image.

Figure 31: Street park condition sketch. Author's own image.

Figure 32: Site Plan. Author's own image.

Figure 33: Section through Eastern Pavilion. Author's own image.

Figure 34: Three diagrams of formal/spatial logic. Author's own image.

Figure 35: Section through site. Author's own image.

Figure 36: Section through Western Pavilion. Author's own image.

Figure 37: Diagram of circulation from park and street. Author's own image.

Figure 38: Location of main divisions and circulation in plan. Author's own image.

Figure 39. Ground floor plan. Author's own image.

Figure 40: First floor plan. Author's own image.

Figure 41: Second floor plan. Author's own image.

Figure 42: Third floor plan. Author's own image.

Figure 43: Fourth floor plan. Author's own image.

Figure 44: Park elevation shown from street. Author's own image.
Figure 45: Sketch of park perspective. Author's own image.

Figure 46: Street elevation perspective. Author's own image.

Flgure 47: Live-work-play drawings. By author.

Figure 48: Plan showing live-work dialogues. Author's own image.

Figure 49: Six sectional diagrams. Author's own image.

Figure 50: Private spaces shown in section. Author's own image.

Figure 51: Public spaces shown in section. Author's own image.

Figure 52: Section through Western pavilion (labelled) Author's own image.

Figure 53: Section through Eastern pavilion (labelled). Author's own image.

Flgure 54: Night view from park. Author's own image.

Figure 55: Exterior view from public forecourt. Author's own image.

Figure 56: Exterior perspective from park in the rain. Author's own image.

Figure 57: Access way to first floor from street. Author's own image.

Figure 58: Inside the live-work chasm. Author's own image.

Figure 59: Section through the live-work chasm in each pavilion. Author's own image.

Figure 60: Interior perspective of lie-work chasm. Author's own image.

Figure 61: Interior perspective looking across live-work chasm. Author's own image

Figure 62: Three plans of one housing cluster. Author's own image.

Flgure 63: A housing 'cluster' with accompanying diagrams. Author's own image.

Figure 64: Partial section through one housing cluster and floors below. Author's own image.

Figure 65: Sketch of section. Author's own image. 
Figure 66: A bed-sit studio apartment, perspective. Author's own image.

Figure 67: Looking out from shared lounge. Author's own image.

Figure 68: Longitudinal section with circle labels. Author's own image.

\section{Part Two: Towards the Sub-centre as Architecture's Project}

Figure 1: Design iteration, perspective from above (title page). Author's own image.

Figure 2: Christchurch CBD Aerial photograph (title page). Adapted from kete.digitalnz.org. Web. 19 May 2013.

Figure 3: Pearl River Delta Urban Agglomeration. Source: Global Metropolitan Observatory. Web. 20 May 2013.

Figure 4: Sub-centre forces diagram. Author's own image.

Figure 5: La Defense Sub-centre. Paris, France. Photograph. Source: Landscape Architecture Study Tour with Professor Jack Ahem. Web. 19 May 2013

Figure 6: Potsdamer Platz, Berlin, Germany. Aerial Photograph. Source:"Low-to-no carbon City: Lessons from western urban projects for the rapid transformation of Shanghai", Habitat International, 37. Web. 20 May 2013.

Figure 7: Zhenru Sub-centre, Shanghai, China (artist's impression). Source: "Low-to-no carbon City: Lessons from western urban projects for the rapid transformation of Shanghai", Habitat International, 37. Web. 20 May 2013.

Figure 8: Plan Voisin, Paris, France. Le Corbusier (1925). Source: Fondation Le Corbusier. Web. 25 May 2013.

Figure 9: Potsdamer Platz, Berlin, Germany. Photograph by Rick Steves. Source: Rick Steves Europe. Web. 25 June 2013.

Figure 10: Chongqing Sub-centre, Shanghai, China. Aerial perspective render. Source: "Chongqing Xiyong integrated planning". Arup Website. Web. 20 May 2013.
Figure 11: Chongqing Sub-centre, Shanghai, China. Perspective render. Source: "Chongqing Xiyong integrated planning". Arup Website. Web. 20 May 2013.

Figure 12: Diagram of Centrality. Author's own image.

Figure 13: Diagram of embodied paradox. Author's own image.

Figure 14: Diagram of many centres. Author's own image.

Flgure 15: Diagram of sub-centre as paradox. Author's own image.

Figure 16: Potsdamer Platz, Berlin, Germany. Aerial Photograph. Source: "Low-to-no carbon City: Lessons from western urban projects for the rapid transformation of Shanghai", Habitat International, 37. Web. 20 May 2013.

Figure 17: Potsdamer Platz, Berlin, Germany. Photograph copyrighted Muhs/Elsen. Source: "'Potsdamer Platz" Stadtentwicklung Berlin. Web. 19 May 2013.

Figure 18: Westfield Shopping Mall, Lower Hutt, Wellington. Photograph by author.

Figure 19: Los Angeles Highway. Photograph. Adapted from: Good Food Preservation. Web. 20 May 2013.

Figure 20: "High-Rise City" by Ludwig Hilberseimer (1924). Drawing. Source: "Experimental practices in architectural, urban, and landscape history, theory, and criticism", Htc Experiments. Web. 25 May 2013.

Figure 21: "The Continuous Monument: New New York" by Superstudio (1969). Collage. Source: Jesse Van Winden Wordpress. Web. 25 June 2013.

Figure 22: San Cataldo Cemetary by Aldo Rossi (1972). Modena, Italy. Photograph. Source: "San Cataldo Cemetery". Mi Modern Architecture. Web. 30 June 2013.

Figure 23: "Delirious New York" (Cover Page), by Rem Koolhaas. Drawing. Source: "Delirious New York - 35 years on". Nyc Architecture, <www. nyc-architecture.com/?p=2152>. Web. 25 June 2013. 
Figure 24: Nagakin Capsule Tower by Kisho Kurokawa (1972). Tokyo, Japan. Photograph. Source: Architectural Moleskin Blogspot. <www. architecturalmoleskine.blogspot.com>. Web. 25 June 2013.

Figure 25: New Babylon by Constant Nieuwenhuys (195974). Drawing. Source: "Constant's New Babylon", <www.benandjess.wordpress com/2008/12/02>. Web. 30 June 2013.

Figure 26: City of Tokyo. Photograph by A. Maslennikov/ Azote. Source: Stockholm Resilience Centre. $<$ http://www.stockholmresilience.org/21/ research/research-news $>$. Web. 26 June 2013.

Figure 27: E.U.R . Rome, Italy. Photograph. Source: Dartmouth, http://www.dartmouth.edu/. Web. 26 June 2013.

Figure 28: Lower Hutt Town Hall by Keith Cook (1957). Wellington, New Zealand. Photograph by author.

Figure 29: Boston City Hall by Gerhard Kallmann (1968). Boston, USA. Photograph. Source: Wikimedia Common,.https://commons.wikimedia.org/ wiki/File:BostonCityHall2.JPG. Web. 26 June 2013.

Figure 30: The Sage, Gateshead, UK. Photograph by Alephnaught. Source: Flickr Commons. www.flickr.com/photos/ portaldeltag/5399314438.>. Web. 26 June 2013.

Figure 31: Selfridges by Daniel Burnham (2003). Birmingham, UK. Photograph by Wojtek Gurak. Source: "Blobitecture - The Rise of Organic Architecture. Kuriositas. http://www. kuriositas.com/2011/01/blobitecture-rise-oforganic.html.>. Web. 27 June 2013.

Figure 32: Diagram of three components of a critical engagement with the city. Author's own image.

Figure 33: House ii by Peter Eisenman (1969-70). Hardwick, Vermont. Photograph. Source: McGill Architecture Website. Web. 27 June 2013.
Figure 34: Peter Eisenman, axonometric sequence of diagram. Source: Architect. http://archinect. com/features/article/2875457/5-projectsinterview-5-alexander-maymind.>. Web. 26 June 2013.

Figure 35: Elliptic City IFCA Masterplan by Bernard Tschumi Architecture (2005). Santo Domingo, Domincan Republic. Source: Bernard Tschumi Architecture, <http://www. tschumi.com/media/files/02038.jpg>. Web. 26 June 2013

Figure 36: An Architecture-city Trialectic. Author's own image.

Figure 37: Diagram of argument. Author's own image.

Figure 38: The City of the Captive Globe Revisited by Rem Koolhaas and Madelon Vriesendorp (1994). New York. Source: <http://3.bp.blogspot. com/-m1GZxfWhUol/T3LL3sCbqMI/ AAAAAAAACXE/URNOY7vNgZ4/ s1600/Koolhaas+\&+Vriesendorp_ Captive+Globe+Revisited_1994_ H5026_72dpi.jpg>. Web. 28 June 2013.

Figure 49: Architecture as Small City drawing. Source: Trummer, Peter. "The City as an Object: Thoughts on the Form of the City." Log 27, 2013: 51.

Figure 40: A Part-to-whole Relationship. Author's own image.

Figure 41: Beko Building by Zaha Hadid Architects (2012). Belgrade, Serbia. Image by Zaha Hadid Architects. Source: Business Insider. http:// au.businessinsider.com/zaha-hadids-bekobuilding-in-belgrade-2012-11.>. Web. 26 June 2013.

Figure 42: El Casa De Musica by OMA (2005). Porto, Lisbon. Photograph. Source: E-Architect., <http://www.e-architect.co.uk/portugal/ casa_da_musica.html.>. Web. 26 June 2013.

Figure 43: El Casa De Musica by OMA (2005). Porto, Lisbon. Elevation. Source: "Casa de Musica", Open Bulidings. <http://openbuildings.com/ buildings/casa-da-musica-profile-1320/ media?group=image >. Web. 27 June 2013. 
Figure 44: El Casa De Musica by OMA (2005). Porto, Lisbon. Photograph of wider urban context. Source: Architecture Page, http://www.architecturepage.com/go/projects/casa-da-musica_all.>. Web. 26 June 2013

Figure 45: Diagrams Studies of Casa de Musica as objectform. Source: < http://nevolution.typepad. com/theories/architexture/>. Web. 27 June 2013.

Figure 46: Diagram of Compositional Tension. Author's own image

Figure 47: Jubilee Churc by, Richard Meier and Partners (2003). Rome, Italy. Photograph. Source: Arch News Now, <http://www.archnewsnow.com/ features/images/Feature0123_02x>. Web. 20 June 2013.

Figure 48: Jubilee Church by Richard Meier and Partners (2003). Rome, Italy. Photogragh of elevation. Source: Esquire, <http://www.esquire.com/ $\mathrm{cm}$ /esquire/images/jubilee-church-meier0908-lg >. Web. 20 June 2013.

Figure 49: Jubilee Church by Richard Meier and Partners (2003). Rome, Italy. Elevations. Source: Arch Daily. <http://www.archdaily.com/20105/ church-of-2000-richard-meier/>. Web. 20 June 2013.

Figure 50: Neue National Gallery by Mies Van Der Rohe (1968). Berlin. Photograph from above. Source: Arch Daily, <www.archdaily.com/3869/neuenational-gallery-in-berlin-mies-van-derrohe/.>. Web. 20 June 2013.

Figure 51: Neue National Gallery by Mies Van Der Rohe (1968). Berlin. Photograph from street. Source: Arch Daily, <www.archdaily.com/3869/neuenational-gallery-in-berlin-mies-van-derrohe/.>. Web. 20 June 2013.

Figure 52: Seagram Building by Mies Van Der Rohe (1958). New York. Source: Tumblr, <http:// www.tumblr.com/tagged/seagram $\% 20$ building?before=74>. Web. 20 June 2013.

Figure 53: Property with Properties by Zago Architecture (2012). Rialto, California. Perspective from above. Source: Domus, <http://www. domusweb.it/content/domusweb/en/ architecture/2012/02/15/-sub-urban-realities. html>. Web 25 May 2013.
Figure 54: Property with Properties by Zago Architecture (2012). Rialto, California. Photograph of design model. Source: Domus, <http:// www.domusweb.it/content/domusweb/en/ architecture/2012/02/15/-sub-urban-realities. html>. Web. 25 May 2013.

Figure 55:, Thoughts on a Walking City by MOS Architects (2012). Orange, New Jersey. Photograph of model, perspective. Source: Design Applause, $<$ http://designapplause.com/wp-content/ xG58hlz9/2012/02/foreclose-orange2.png.>. Web. 26 May 2013.

Figure 56:, Thoughts on a Walking City by MOS Architects (2012). Orange, New Jersey. Photograph of model, plan. Source: Domus, <http://www. domusweb.it/content/domusweb/en/ architecture/2012/02/15/-sub-urban-realities. html>. Web. 25 May 2013.

Figures 57-60: EWHA Women's University by Dominque Perrault Architecture (2008). Seoul, South Korea. Photographs. Source: Arch Daily, <http:// www.archdaily.com/227874/ewha-womansuniversity-dominique-perrault-architecture/ ufe_0805-ext_am-02/>. Web. 20 May 2013.

Figure 61: Oslo Opera House by Snøhetta, (200\&). Oslo, Norway. Photograph. Source: About Architecture, <http://architecture.about. com/od/theaters/ig/Theaters-/Oslo-OperaHouse.htm>. Web. 20 May 2012.

Figure 62: Comparison of two approaches. Author's own image.

Figure 63: Four ideas of the sub-centre. Author's own image.

Figure 64: Healthcare Centre For Cancer Patients by Nord Architects (2010). Copenhagen, Denmark.. Photograph from street. Source: Dezeen, < http://www.dezeen.com/2012/03/28/ healthcare-centre-for-cancer-patients-bynord-architecture/>. Web. 26 May 2013.

Figure 65: Healthcare Centre For Cancer Patients by Nord Architects (2010). Copenhagen, Denmark. Photograph from above. Source: Dezeen < http://www.dezeen.com/2012/03/28/ healthcare-centre-for-cancer-patients-bynord-architecture/>. Web. 26 May 2013.

Figure 66: Retail Precinct by RTA Studio (2012), Auckland, New Zealand. Photograph of street elevation. Source: Architecture Now, <http:// architecturenow.co.nz/articles/blue-skythinking/>. Web. 20 May 2013. 
Figure 67: Retail Precinct by RTA Studio (2012), Auckland, New Zealand. Photograph from side. Source: Architecture Now, <http://architecturenow. co.nz/articles/blue-sky-thinking/>. Web. 20 May 2013.

Figure 68: City of Culture by Peter Eisenman Architects (2011). Galicia, Spain. Aerial photograph. Source: Architzer, <http://www.architizer. com/en_us/blog/dyn/35107/niemeyercenter-closes/\#.Ue_182eVDnQ.>. Web. 21 May 2013.

Figure 69: Rolex Learning Centre by SAANA (2009). Lausanne, Switzerland. Photograph. Source: Design Boom, <http://www.designboom. com/architecture/sanaa-rolex-learningcenter/>. Web. 25 May 2013.

Figure 70: Die Bremer Stadtmusikanten by ARTEC Architects with Neues Lebel housing association (2009). Vienna, Austria. Photograph. Source: Arch Daily, <http:// www.archdaily.com/262669/terrace-housetokiostrasse-artec-architekten/ .> . Web. 26 May 2013.

Figure 71: The Sub-centre as Architecture's Project. Author's own image.

Figure 72: An open ended conclusion for the sub-centre (argument structure). Author's own image.

\section{Design Exegesis and Conclusion}

Figure 1: Matrix of Sketches (Title page). Author's own image.

Figure 2: Matrix of formal strategy diagrams. Author's own image.

Figure 3: Process sketches of part to whole. Author's own image.

Figure 4: Matrix of part to whole investigations. Author's own image.

Figure 5: Development derived from grain - massing study. . Author's own image.

Figure 6: Elevational study.. Author's own image.
Figure 7: Collage of Housing in an informal arrangement. Author's own image.

Figure 8: Massing study of part to whole. Author's own image.

Figure 9: Massing study zoom in. Author's own image.

Figure 10: Striations in ground plan - sketch. Author's own image.

Figure 11: Sketch of repeated elements 1. Author's own image.

Figure 12: Sketch of repeated elements. 2. Author's own image.

Figure 13: Figure ground and basic massing study. Author's own image.

Figure 14: Series of formal studies testing park/street address.

Figure 15: Linking through to park and street - process render. Author's own image.

Figure 16: Render with incisions into ground plane. Author's own image.

Figure 17: Series of five typology studies. Author's own image.

Figure 18: Matrix of sketches exploring typological advance. Author's own image.

Figure 19: A layered grandstand iteration. Author's own image.

Figure 20: A series of pavilion studies in a row. Author's own image.

Figure 21: Elevational studies and voided forms (sketches). Author's own image.

Figure 22: Programmatic overlaps render. Author's own image.

Figure 23: Site elevation - early iteration. Author's own image.

Figure 24: Massing study of programmatic overlaps. Author's own image.

Figure 25: Public porch render. Author's own image.

Figure 26: Series of studies into 'cutting' on type from another. Author's own image. 
Figure 27: Perspective of design iteration showing clear 'cut' between types. Author's own image.

Figure 28: Section from early design iteration exploring three types. Author's own image.

Figure 29: Elevation from early design iteration. Author's own image.

Figure 30: Articulating the interstitial spaces: a series of experiments. Author's own image.

Figure 31: Massing model with diagrams overlayed. Photograph. Photograph by author.

Figure 32: Sectional studies of interstitial space (sketches). Author's own image.

Figure 33: Process renders showing investigations into interstitial space. Author's own image.

Figure 34: "Formalizing the Transition: between park and street". Sketch. Author's own image.

Figure 35: "A Forged Familiarity". Site plan render. Author's own image.

Figure 36: "Negotiating Public Space". Sectional diagram. Author's own image.

Figure 37: An Embodied Tension". Perspective render Author's own image.

Figure 38: "A Resolute Fabric." Street render. Author's own image.

Figure 39: Diagram of live-work collision as architectural potential. Author's own image.

Figure 40: “Formalizing New Habitations”. Render. Author's own image.

Figure 41: "Exploiting Live-work dialogues". Render. Author's own image.

Figure 42: "A New Response". Perspective Render. Author's own image.

Figure 43: "A Public Porch". Render. Author's own image.

Figure 44: "A Project on the Sub-centre". Composite image of design, sub-centre ideas, formal strategies and Sumner. Authors own image.

Figure 45: "A Design Project on the Sub-centre". Matrix of process Images. Authors own image.
Figure 46: "Towards a Polycentric Christchurch". Adapted from: Map, Koordinates. Web. 20 February 2013.

Figure 47: Sectional Sketch. Authors own image. 
